Society of Urodynamics, Female Pelvic Medicine \& Urogenital Reconstruction 2014 Winter Meeting February 25-March 1, 2014 Doral Golf Resort \& Spa Miami, Florida 


\section{PODIUM ABSTRACTS}

\section{Podium \#1}

THULIUM LASER VAPORIZATION OF THE PROSTATE: SHORT-TERM OUTCOMES OF A SIMPLE STANDARDIZED TECHNIOUE

Joseph J. Pariser, $M^{1}$, Olufenwa F. Milhouse, $M D^{1}$, Shane M. Pearce, $M^{1}$, Leah C. Anderson $\mathrm{PA}^{2}$ and Doreen E. Chung, $\mathbf{M D}^{1}$

${ }^{1}$ Section of Urology, University of Chicago Medical Center, Pritzker School of Medicine, Chicago, IL; ${ }^{2}$ Division of Urology, Mt. Sinai Hospital, Chicago, IL

(Presented by: Joseph J. Pariser, MD)

Introduction: The thulium laser was introduced in 2005 for treatment of BPH. Enucleation studies from outside North America show comparable efficacy and lower morbidity to transurethral resection of the prostate. Few studies exist describing outcomes of vaporization, the most commonly used technique for most urologists. We present our 12 month outcomes of transurethral thulium vaporization of the prostate (ThuVP) with a simple standardized technique.

Methods: From December 2010 to May 2013, 66 men underwent ThuVP using the CyberTM ${ }^{\mathbb{R}}$. Data was collected on demographics, comorbidities, intraoperative measures, complications, serum parameters, maximum flow rate (Omax), postvoid residual (PVR), International Prostate Symptom Score (IPSS), quality of life score (OoL), and PSA. Patients were evaluated at 1 , 3,6 , and 12 months postoperatively. 9 patients were excluded for known prostate cancer diagnosis. Statistical analysis was done using Wilcoxon signed rank test.

Results: Mean age was $66 \pm 10$ years with mean prostate size $59.0 \pm 38.4 \mathrm{cc}$. At baseline, mean IPSS was $20 \pm 8$. OoL score was $4 \pm 1$, Omax was $5.2 \pm 4.5 \mathrm{~mL} / \mathrm{sec}$, and PVR was $228 \pm 402 \mathrm{~mL}$. Median Charlson Comorbidity Index was 3 (IOR 2-5). 27 (47\%) patients were on oral anticoagulation. Mean laser time was $35 \pm 18$ minutes and energy used was $235 \pm 141 \mathrm{~kJ}$. Mean change in hemoglobin was $0.7 \mathrm{~g} / \mathrm{dL}$. There was no significant change in sodium from baseline. Mean hospital stay was $0.4 \pm 0.9$ days with 45 (79\%) patients discharged the same day as surgery. Mean catheter time was $2.9 \pm 2.6$ days. 2 (4\%) patients developed urethral strictures, which were managed endoscopically. There were 4 (7\%) post-operative UTIs. No other major complications were encountered, and no transfusions were required. No patients underwent repeat vaporization for bleeding or regrowth. Significant improvements from baseline were seen in Omax, PVR, IPSS, and OoL score at each time interval (see chart). All 13 patients who were in retention preoperatively were voiding freely last follow-up.

Conclusion: Our Results suggest that thulium laser vaporization of the prostate is a safe and effective outpatient technique for treatment for BPH with durable outcomes at 1 year.

Clinical Relevance: BPH

Mean voiding parameters and PSA at baseline and follow-up
\begin{tabular}{|l|l|l|l|l|l|}
\hline & Baseline & 1 Month & 3 Months & 6 Months & 12 Months \\
\hline IPSS & 20.1 & $10.6^{*}$ & $8.7^{*}$ & $6.2^{*}$ & $7.4^{*}$ \\
\hline QoL & 4.5 & $2.3^{*}$ & $2.1^{*}$ & $1.9^{*}$ & $1.9^{*}$ \\
\hline Qmax & 5.2 & $16.2^{*}$ & $14.8^{*}$ & $16.5^{*}$ & $16.0^{*}$ \\
\hline PVR & 228 & $45^{*}$ & $50^{*}$ & $50.5^{*}$ & $36.3^{*}$ \\
\hline PSA & 2.4 & & $1.76^{*}$ & $1.85^{*}$ \\
\hline
\end{tabular}
* $<<0.05$ when compared to baseline

Published online in Wiley Online Library (wileyonlinelibrary.com). DOI 10.1002/nau.22577

\section{Podium \#3}

\section{MEASUREMENT OF BASELINE AND}

PREVIOUSLY-UNDIAGNOSED COGNITIVE DECLINE IN OLDER ADULTS PRESENTING FOR UROLOGIC EVALUATION

Gray Roberge, Kirsten Greene, MD, MS, Donna Deng, MD, MS

Univ. of California, San Francisco, San Francisco, CA

(Presented by: Gray Roberge)

Introduction and Objectives: Given concerns of side effect profiles of medications and other therapeutic modalities, it is important to know the baseline cognitive status of our patients. We hypothesized that mild cognitive impairment (MCI) is largely undiagnosed. This study measured incidence of $\mathrm{MCI}$ in adults presenting for urologic evaluation and to what extent MCI was undiagnosed.

Methods: We enrolled patients $>18$ years of age presenting to the UCSF Urology Faculty Practice and Urologic Oncology clinics for a wide variety of urological complaints. To determine cognitive status, patients were administered the Montreal Cognitive Assessment (MoCA) screening test. A score of 26-30 on the MoCA is considered cognitively normal, while $<26$ indicates MCI. Patient charts were reviewed for previous diagnoses of neurological decline or pathology.

Results: A total of 83 patients were enrolled. 14 patients were not included because of incomplete or unreliable MoCA administrations. The mean MoCA score for patients age 18$64(n=35)$ and older adults $(n=34)$ was $25.74 \pm 0.3897$ and $23.09 \pm 0.4554$, respectively $(p<.0001)$. From chart review of patients $<64$ years, only 2 possessed prior documentation of neuropathologies (chronic hepatic encephalopathy, stroke, cerebral neoplasms). Of older adults (age $>65$ ), only 5 carried diagnosis of neural disorder (2 with memory loss; one selfreported memory problems; and one each of subdural hematoma and stroke).

Conclusion: These initial data demonstrate significant incidences of MCI that are previously undiagnosed in adults undergoing urologic evaluation, with larger proportions of MCI appearing in older adults. We do acknowledge potential confounders that may artificially lower MoCA scores, including patients feeling nervous, varying location of MoCA administration, or providers entering the room during test administration. Still, we believe this study is an important initial step in illuminating potential cognitive barriers with patients. Once further explored, this knowledge will permit better communication with patients and more appropriate pre- and postoperative instructions that will increase patient safety and improve outcomes.

Clinical Relevance: Geriatric Urology

\begin{tabular}{|l|c|c|c|c|c|c|c|c|}
\hline Group Age Range, Years & $18-24$ & $25-34$ & $35-44$ & $45-54$ & $55-64$ & $65-74$ & $75-84$ & $85-94$ \\
\hline \#Patients with MoCA Score <26 & 1 & 2 & 4 & 1 & 6 & 17 & 9 & 3 \\
\hline Total Patients in Age Group & 2 & 7 & 8 & 4 & 14 & 22 & 9 & 3 \\
\hline \% with Mild Cognitive Impairment & $50 \%$ & $29 \%$ & $50 \%$ & $25 \%$ & $43 \%$ & $77 \%$ & $100 \%$ & $100 \%$ \\
\hline $\begin{array}{l}\text { \# Patients with Already-Diagnosed } \\
\text { Neurclogical Impairment/Pathology }\end{array}$ & 0 & 0 & 0 & 0 & 2 & 4 & 1 & 0 \\
\hline
\end{tabular}

\section{Podium \#4}

NOCTURNAL POLYURIA IN OLDER FEMALES WITH URGE URINARY INCONTINENCE: ROLE OF SLEEP, TIME IN BED AND MEDICATIONS USED

Shachi Tyagi, MD, Subashan Perera, PhD, Stasa Tadic, MD, Neil Resnick, MD

Pittsburgh, PA

(Presented by: Shachi Tyagi, MD)

(C) 2014 Wiley Periodicals, Inc. 
Introduction: Nocturia is one of the most common and bothersome lower urinary tract symptoms (LUTS). Nocturnal polyuria is often cited as a major contributing factor to the etiology of nocturia, but the effect of comorbid conditions and polypharmacy on nocturnal urine production in older adults with LUTS is not known.

Objective: To identify potential correlates of nocturnal polyuria among older females with urge urinary incontinence (UUI). Method: Secondary data analysis was performed of a prospective study that assessed the factors predicting and mediating the response of UUI to biofeedback therapy. Baseline data from 284 females, mean age $72.9 \pm 7.9$ years, was analyzed. All had completed 3-day voiding diary that included time of voiding, voided volumes, and circumstances of leakage. Available data also included all diagnoses, medications, and physical exam. Nocturia was defined as waking at least once per night to void and nocturnal polyuria (NP) as nocturnal urinary volume (NUV, including the first morning void) greater than 33\% of 24-hour volume. The sleep restlessness question from the Center for Epidemiologic Studies Depression Scale was used as a proxy measure for sleep quality.

Results: $35 \%$ of subjects did not have nocturia. $75 \%$ of women with nocturia had NP. Those with NP spent significantly more time in bed (532 \pm 71 minutes) than did nocturics without NP (479 \pm 57 minutes, $p<.0001)$, or those without nocturia (461 \pm 71 minutes, $p<.0001$ ). Participants with higher nocturnal volume reported waking earlier to void and having worse sleep quality despite spending more time in bed. In multivariable regression analysis, age, body mass index, and time spent in bed, were independent predictors of NP in nocturics, while use of an anti-depressant and use of angiotensinconverting enzyme inhibitors ACE-I/angiotensin receptor blockers (ARBs) were independently protective. Edema was not associated with NP in either bivariate or multivariate analysis

Conclusion: Among older females with UUI and nocturia, optimizing BMI and restricting the time spent in bed may help improve NP, and should be further studied as potential intervention targets. Use of anti-depressants and ACE-I/ARB, when appropriate, may benefit NP.

Support: T32 AG021885, AFAR, The John A. Hartford Foundation and the Centers of Excellence National Program, 2R01AG020629-06, 5K23AG31916

Clinical Relevance: Geriatric Urology

\section{Podium \#5 \\ SEGMENTAL HYPERALGESIA TO MECHANICAL STIMULUS IN INTERSTITIAL CYSTITIS/BLADDER PAIN SYNDROME - EVIDENCE OF CENTRAL SENSITIZATION}

H. Henry Lai, $M^{1}$, Vivien Gardner ${ }^{1}$, Timothy Ness, $M D, \mathrm{PhD}^{2}$ and Robert Gereau, IV, $\mathrm{PhD}^{1}$

${ }^{1}$ Washington University School of Medicine, St Louis, MO;

${ }^{2}$ University of Alabama at Birmingham, Birmingham, $A L$

(Presented by: H. Henry Lai, MD)

Objectives: Interstitial cystitis/bladder pain syndrome (IC/BPS) is characterized by hypersensitivity to bladder distention. However, it is unclear if IC/BPS patients have generalized hyperalgesia outside the pelvis. The Objectives: of this study are to investigate if: (1) IC/BPS subjects demonstrate mechanical or thermal hyperalgesia, and (2) whether the hyperalgesia is segmental (more pronounced in T10-T12 or S2-S4) or generalized/global (involving the upper and lower extremities also).
Materials and Methods: 10 female IC/BPS subjects and 10 agematched female controls without co-morbid fibromyalgia or narcotic use were recruited for quantitative sensory testing. Using the Method of Limits, pressure pain and heat pain thresholds were measured. Using the Method of Fixed Stimulus, the visual analog scale (VAS) pain experienced was recorded when a fixed pressure ( $2 \mathrm{~kg}$ and $4 \mathrm{~kg}$ ) or temperature $\left(35^{\circ} \mathrm{C}\right.$ was $37^{\circ} \mathrm{C}$ ) was applied. The body sites tested included: (1) T1: upper extremity, (2) T11: suprapubic, (3) L4: lower extremity, (4) S2: sacral dermatome, and (5) S3: perineum.

Results: The VAS pain rated by female IC/BPS subjects was significantly higher than the VAS pain rated by female control subjects when a fixed mechanical pressure ( $2 \mathrm{~kg}$ or $4 \mathrm{~kg}$ ) was applied to the suprapubic (T11) area ( $p=0.028$, two-way ANOVA group difference). There was an up-shift of the stimulus-response curve, which corresponded to the presence of mechanical hyperalgesia in the suprapubic area in IC/BPS. However, the VAS pain rated by IC/BPS subjects was not different from those rated by controls when a fixed pressure was applied at the other body sites (T1 arm, L4 leg, S2-3 sacral). No difference in VAS pain rating was noted when a fixed heat stimulus $\left(35^{\circ} \mathrm{C}\right.$ or $\left.37^{\circ} \mathrm{C}\right)$ was applied to any of the body sites tested (T1, T11, L4, S2). There was no difference in pressure pain thresholds or thermal pain thresholds between IC/BPS and controls.

Conclusion: The present study demonstrated that IC/BPS is characterized by psychophysical evidence of hypersensitivity to mechanical pressure applied to the suprapubic area. Segmental hyperalgesia to mechanical pressure stimulation was demonstrated in the suprapubic area (T10-T12) but not in sacral area (S2-S4) or in upper and lower extremities. This segmental hyperalgesia may be explained in part by spinal central sensitization.

Support: NIH NS048620, DK094964

Clinical Relevance: Female Urology-including Incontinence

\section{Podium \#6 \\ OUTCOME OF VAGINAL MESH OR TAPE REMOVED TRANSVAGINALLY FOR PAIN ONLY}

Jack C. Hou, MD, Feras Alhalabi, MD, Philippe E. Zimmern, MD UT Southwestern Medical Center, Dallas, TX

(Presented by: Jack C. Hou, MD)

Introduction: Because there is reluctance to operate for pain, we evaluated mid-term outcomes of vaginal mesh $(M)$ or synthetic suburethral tape $(\mathrm{T})$ removed for pain as the only indication.

Material \& Methods: Following IRB approval, a prospective database on non-neurogenic women operated for $M$ or $T$ removal, with the focus on pain as the only and single reason for removal, and a minimum 6-month follow-up, was reviewed. Primary outcome was pain level assessed by Visual Analog Scale (VAS) (0-10) at baseline and at each subsequent visit as documented in the electronic medical record upon arrival, with the last visit VAS score used for this analysis. Parameters evaluated included demographics, mean time to presentation, and type of $\mathrm{M}$ or $\mathrm{T}$ inserted.

Results: From 2005-2013, 123 patients underwent surgical removal (69 M, $54 \mathrm{~T}$ ) with pain as the only indication (Table 1$)$. Mean follow up was 35 month for the T group (6-59) and 22 month for the $M$ group (6-47). VAS dropped from mean preoperative level of 7.9 to 0.9 after surgery $(p=0.0014)$ in the $M$ group, and from 5.3 to 1.5 after surgery $(p=0.00074)$ in the $T$ group. Impressively, pain-free status (VAS score=0) was 
achieved in $81 \%$ of $\mathrm{T}$ and $67 \%$ of $M$. In the pain persistent group, there was no difference in mean time to presentation since the placement surgery, with 36.2 month (31-42) for the T group and 37.5 month (32-39) for the $M$ group $(p=0.62)$. No statistically significant difference was found between $\mathrm{T}$ and M groups. (Table 1)

Conclusion: When pain is the sole indication for $\mathrm{T}$ or $M$ removal, a significant reduction in pain score can be durably expected after removal in the majority of patients at mid-term follow-up.

\begin{tabular}{|c|c|c|c|}
\hline \multicolumn{4}{|c|}{ 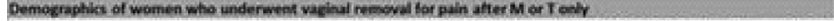 } \\
\hline & Trans-Vaginal Mevh Gemoval & Trans: Watinal Tape Remaval & PValue \\
\hline & Na 69 & $N=54$ & \\
\hline Mean Age (Fest) & $49(41-63)$ & 53(34-72) & $0,0 \%$ \\
\hline Mexa BML $\left(\mathrm{ka} / \mathrm{m}^{2}\right)$ & $30[23-39$ & $27(24-36)$ & 0.8 \\
\hline Meua Pre-operative & 79 & \$3 & 0.048 \\
\hline \multicolumn{4}{|l|}{ Fain score VAs } \\
\hline \multicolumn{4}{|l|}{$(0-10)$} \\
\hline Mean Post-operative & a.9 & 1.5 & 0.07 \\
\hline \multicolumn{4}{|l|}{$\begin{array}{l}\text { Pain Seere VAS at last visit } \\
\text { (0-10) }\end{array}$} \\
\hline $\begin{array}{l}\text { pain free } \\
\text { Mas=ol) }\end{array}$ & Na46 & Ne44 & N/A \\
\hline $\begin{array}{l}\text { Pain perristent } \\
\text { (no change in VAS) }\end{array}$ & $N=11$ & $\mathrm{~N}=3$ & W/A \\
\hline \multirow{3}{*}{ 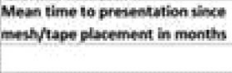 } & $33(1066)$ & $29(8-72)$ & 0,71 \\
\hline & & & \\
\hline & Mrih Muteris] (MN/X) & Tabe Maserial $(\mathrm{B} / \mathrm{x})$ & \\
\hline prolitit & $27 / 39 \%$ & & \\
\hline Devate & $14 / 21 \%$ & & \\
\hline Aroulta & $12 / 16 \mathrm{k}$ & & \\
\hline Merigeed/Aposee & $6 / \% \mathrm{~K}$ & & \\
\hline Unknewes & $10 / 15 \%$ & & \\
\hline 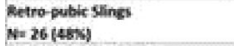 & & $26 / 46 \pi$ & \\
\hline Trans-obturater Sines: & & $21 / 39 \%$ & \\
\hline Mini-slings & & $3 / 5 \%$ & \\
\hline Unkingeren & & $4 / 5 \mathrm{~S}$ & \\
\hline
\end{tabular}

\section{Podium \#7}

LOCAL ANESTHETIC VERSUS SALINE TRIGGER POINT INJECTIONS FOR MYOFASCIAL PELVIC PAIN: A RANDOMIZED TRIAL

Benjamin Barenberg, $\mathrm{MD}^{1}$, Sondra Summers, $\mathrm{MD}^{2,3}$, Vance Broach, $\mathrm{MD}^{2,3}$, Lyndsey Day, $\mathrm{MD}^{2,3}$

and Kimberly Kenton, $\mathrm{MD}, \mathrm{MS}^{2,3}$

${ }^{1}$ Loyola University Stritch School of Medicine, Maywood, IL; ${ }^{2}$ Loyola University Stritch School of Medicine; ${ }^{3}$ Department of Obstetrics and Gynecology, Chicago, IL

(Presented by: Benjamin Barenberg, MD)

Introduction: To determine if pelvic floor trigger point injections(TPI)with bupivacaine provide better pain relief than TPI with saline in women with myofascial pelvic pain syndrome(MPPS).

Methods and Materials: We enrolled women with MPPS in a double blind randomized trial of pelvic floor TPI with bupivacaine or saline. Exclusion criteria were pregnancy, prior pelvic TPI, and clinically active pain syndromes. Participants were randomized to TPI with saline or $0.25 \%$ bupivacaine with a maximum of 6 TPI and $18 \mathrm{ml}$ of study solution. Demographics, pre and post-injection Interstitial Cystitis Severity and Problem Index(ICSI/ICPI), and an Irritable Bowel Severity Score (IBSS) were collected along with a pre-injection, 15 minute, 1 week, and 6 week post-injection pain Numeric Rating Scores (NRS) and a Global Impression of Improvement(GPI).

Results: Primary outcome was NRS at 15 minutes postinjection. Based on previous data(1), it is assumed saline will impart analgesic benefit. Therefor, a student t-test for independent samples required 8 subjects per group to provide $80 \%$ power at alpha $=0.05 .17$ women, 9 bupivacaine and 8 saline, were randomized and underwent TPI. Participants had a mean age of $45 \pm 15$ years and BMI of $27 \pm 7 \mathrm{~kg} / \mathrm{m} 2$. No differences were noted between groups, including age, BMI, smoking status, sexual activity, current medical therapy, history of pelvic floor therapy, or number of TPI. There was no significant difference in NRS between women receiving saline or bupivacaine TPI at $15 \mathrm{~min}, 1$ week, or 6 weeks; however, NRS improved for the entire study population at all time points(Table 1). Saline trended toward a significant improvement of NRS at 6 weeks, while Bupivacaine showed significant improvement in NRS at weeks 1 and 6. Global Impression of Improvement improved in both arms, but did not differ amongst groups $(p=.42)$. The ICSI/ICPI and IBSS did not improve at any time point. Pelvic floor TPI provide significant improvement in NRS. Conclusion: Bupivacaine is not superior to saline in improving pelvic pain NRS at 15 min post-injection. Perceived improvement is positive in both groups and agrees with research establishing a minimal clinically important difference in posttreatment pelvic pain scores of 10 with a mean change of 18(2). Clinical Relevance: IC and Pelvic Pain-UTI / Inflammatory

\begin{tabular}{|c|c|c|c|c|c|c|c|}
\hline I & $\begin{array}{l}\text { Baseline NRS } \\
\text { MeantSD }\end{array}$ & $\begin{array}{l}15 \min \text { NRS } \\
\text { Meantso }\end{array}$ & $\begin{array}{l}1 \text { week NFs } \\
\text { MeantSD }\end{array}$ & $\begin{array}{l}6 \text { weekNRS } \\
\text { MeantSD }\end{array}$ & $\begin{array}{l}\text { NRS } \\
\text { Baseline } \\
\text { and 15 } \\
\text { min P } \\
\text { Value }\end{array}$ & $\begin{array}{l}\text { NRS } \\
\text { Baseline } \\
\text { and } 1 \\
\text { weekP } \\
\text { value }\end{array}$ & $\begin{array}{l}\text { NRS } \\
\text { Baseline } \\
\text { and } 6 \\
\text { weekP } \\
\text { Value }\end{array}$ \\
\hline $\begin{array}{l}\text { Salinet } \\
\text { Bupivicaine } \\
\text { (N=17) }\end{array}$ & $53.2 \pm 21.1$ & $37.4 \pm 17.1$ & $30.0 \pm 23.1$ & $32.7 \pm 25.2$ & .035 & .007 & .001 \\
\hline Saline $(\mathrm{N}=8)$ & $55.6 \pm 21.8$ & $42.5+14.9$ & $34.3 \pm 25.9$ & $36.0 \pm 29.7$ & .227 & .123 & .060 \\
\hline $\begin{array}{l}\text { Bupivicaine } \\
(\mathrm{N}=9)\end{array}$ & $51.1+21.5$ & $32.8 \pm 18.4$ & $26.3 \pm 21.4$ & $30.6 \pm 24.0$ & . 107 & . .040 & .006 \\
\hline $\begin{array}{l}\text { PValue } \\
\text { (Saline ys } \\
\text { Bupivacaine) }\end{array}$ & .674 & .253 & 521 & .726 & & & \\
\hline
\end{tabular}

\section{Podium \#8}

\section{CHARACTERIZATION OF A SUBSET OF UROLOGIC CHRONIC PELVIC PAIN SYNDROME (UCPPS) PATIENTS WITH A POLY-SYMPTOMATIC, POLY-SYNDROMIC PATTERN OF PRESENTATION}

H. Henry Lai, MD $^{1}$, Carol S. North ${ }^{2}$ and Hong A. Barry ${ }^{1}$

${ }^{1}$ Washington University School of Medicine, St Louis, MO; ${ }^{2}$ The North Texas VA Health Care System and The University of Texas Southwestern Medical Center, Dallas, TX

(Presented by: H. Henry Lai, MD)

Objectives: Patients with urologic chronic pelvic pain syndrome (UCPPS), which includes interstitial cystitis/bladder pain syndrome or chronic prostatitis/chronic pelvic pain syndrome, commonly report bothersome symptoms outside the genitourinary tract. We have previously shown that about one out of four UCPPS patients reported high somatic symptom burden and a wide symptom distribution across multiple organ systems fitting a "poly-symptomatic, poly-syndromic"(PSPS) pattern of presentation. [J Urol, 187: 2106, 2012] The objective of this study is to characterize this subgroup of PSPS in terms of their urologic pain, symptoms, quality of life, and treatmentseeking behaviors.

Materials and Methods: 81 UCPPS patients enrolled in the NIDDK MAPP Research Network Study at the Washington University in St. Louis and the University of Alabama sites were asked to complete a somatic symptom questionnaire (PSPS-O) to assess their somatic symptom burden and its distribution, the Genitourinary Pain Index (GUPI) to assess their UCPPS symptoms and impact on quality of life, and self-reported treatment-seeking behaviors for their UCPPS symptoms. The 
PSPS pattern in UCPPS patients was defined as self-report of 25 or more painful and non-painful somatic symptoms in 9 or more symptom categories on the PSPS questionnaire.

Results: $25 \%(n=20)$ of UCPPS subjects have PSPS symptom pattern with numerous somatic symptom and wide symptom distribution across multiple organ systems. UCPPS patients with the PSPS pattern reported more severe pain on a Likert scale $(6.3 \pm 2.1$ vs $5.1 \pm 2.3, p=0.04)$, more frequent pain in the past week ( $3.7 \pm 1.3$ vs $2.8 \pm 1.3, p=0.04$ ), and more widespread distribution of the pain in the genital and pelvic areas $(5.8 \pm 2.0$ vs $4.0 \pm 1.9, p=0.008)$ compared to UCPPS patients without PSPS. PSPS patients also had significantly worse scores on the pain subscale ( $15.8 \pm 4.8$ vs $11.9 \pm 4.5, p=0.006)$, quality of life subscale (poorer QOL, $9.2 \pm 2.6$ vs $7.0 \pm 2.8, p=0.003$ ), and total scores (31.4 \pm 8.9 vs $23.3 \pm 8.6, p=0.003)$ on the GUPI questionnaire than patients without PSPS. Patients with the PSPS pattern reported significantly more treatment-seeking behaviors than other patients $(p=0.04)$.

Conclusion: The PSPS pattern might be an important phenotypic factor to assess in the evaluation of UCPPS with clinical and research implications. This may be a distinct clinical subgroup among UCPPS patients with worse pain, OOL and more health utilization.

Funding: NIDDK MAPP Research Network

Clinical Relevance: Female Urology-including Incontinence

\section{Podium \#9}

\section{COMPARISON OF ROC CURVES FOR PREOPERATIVE VLPP, MUCP AND URINARY NTX AS PREDICTORS FOR MIDURETHRALSLING OUTCOMES}

AeuMuro G. Lake, $\mathrm{MD}^{1}$, Pamela Moalli, $\mathrm{MD}, \mathrm{PhD}^{2}$, Holly E. Richter, MD, $\mathrm{PhD}^{3}$, Hae-Young Kim, PhD, MS, $\mathrm{MA}^{4}$, Charles W. Nager, $M^{5}$, Larry Sirls, $M^{6}{ }^{6}$, Linda Brubaker, $M^{7}$, John W. Kusek, $\mathrm{PhD}^{8}$ and Toby C. Chai, $\mathrm{MD}^{1}$

${ }^{1}$ Departments of Urology and Obstetrics, Gynecology\&

Reproductive Sciences, Yale University School of Medicine, New Haven, CT; ${ }^{2}$ Department of Obstetrics, Gynecology and Reproductive Sciences, University of Pittsburgh, Pittsburgh, PA; ${ }^{3}$ Department of Obstetrics and Gynecology, University of Alabama at Birmingham, Birmingham, AL; ${ }^{4} \mathrm{New}$ England Research Institutes, Watertown, MA; ${ }^{5}$ Department of Reproductive Medicine, University of California San Diego, San Diego, CA; ${ }^{6}$ William Beaumont Hospital, Royal Oak, MI;

${ }^{7}$ Department of Urology, Loyola University, Chicago, IL; ${ }^{8}$ NIDDK, Bethesda, $M D$

(Presented by: AeuMuro G. Lake, MD)

Introduction: Receiver operating characteristic (ROC) curves for pre-operative Valsalva leak point pressure (VLPP) and maximum urethral closure pressure (MUCP) showed poor sensitivity and specificity for midurethral sling (MUS) surgical outcomes. Recently, we showed that lower pre-operative urinary NTx (N-terminal telopeptide of crosslinked Type 1 collagen, a marker of collagen turnover) was associated with lower odds of MUS failure (OR 0.49, $p=0.03$ ). We compare ROC curves generated from preoperative VLPP, MUCP, and NTx values obtained from two Urinary Incontinence Treatment Network trials, Trial of Mid-Urethral Slings (TOMUS) and The Value of Urodynamic Evaluation (ValUE).

Methods: "Success"was defined at 12 months post-operatively as a subject having: a 70\% decrease in Urogenital Distress Inventory (UDI) score, a score of 1 or 2 on Patient Global Impression of Improvement, and a negative stress test. Preoperative VLPP and MUCP's were measured in 427 TOMUS subjects; preoperative NTx value was measured in 150 ValUE subjects. ROC curves for VLPP, MUCP and NTx were generated and areas under the curve (AUC) were compared by largesample test method.

Results: Comparison of subject characteristics from TOMUS versus ValUE revealed several significant differences. TOMUS subjects were 2.5 years older $(p=0.01), 1.4 \mathrm{~kg} / \mathrm{m} 2$ heavier $(p=0.01)$ and scored 6.7 points higher on UDI $(p=0.001)$ than ValUE subjects. These factors may have contributed to TOMUS subjects having a lower success rate compared to ValUE subjects $(66.3 \%$ versus $76.0 \%, p=0.03)$. ROC curves for VLPP, MUCP and NTx are shown in Figures $1 \mathrm{~A}-1 \mathrm{C}$, with AUC of 0.542 , 0.561 and 0.702, respectively. The AUC for NTx was significantly higher than VLPP $(p=0.02)$ and MUCP $(p=0.03)$.

Conclusion: Urinary NTx, a non-invasive test, performed superiorly to VLPP and MUCP as a prognostic tool for postMUS surgical outcome. AUC values for VLPP and MUCP fall into the "fail"or "F" category (0.50-0.60), whereas the AUC value for NTx falls into "fair" or "C"category (0.70-0.80). Understanding the pathophysiology associated with how urinary NTx correlates to surgical outcomes represents the next phase to possibly improve surgical management of SUI.

Grant NIDDK U01-DK60397-8S2

Clinical Relevance: Female Urology-including Incontinence
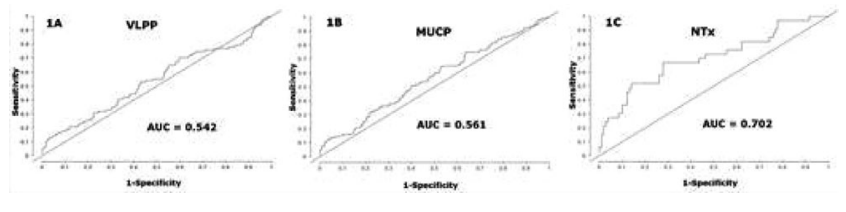

\section{Podium \#10}

PATIENT PERCEPTION OF PHYSICAL AND EMOTIONAL DISTRESS RELATED TO URODYNAMICS TESTING AT THE UNIVERSITY OF MICHIGAN; A OUESTIONNAIRE-BASED STUDY AMONG MEN AND WOMEN WITH AND WITHOUT NEUROLOGOIC CONDITIONS

Anne M. Suskind, MD, $\mathrm{MS}^{1}$, J. Quentin Clemens, $\mathrm{MD}, \mathrm{MS}^{2}$, John T. Stoffel, $M^{2}$, Ann Oldendorf, $M^{2}$, Bahaa S. Malaeb, $\mathrm{MD}^{2}$, Teresa Jandron, $\mathrm{RN}^{2}$ and Anne P. Cameron, $\mathrm{MD}^{2}$

${ }^{1}$ University of Michigan, Ann Arbor; ${ }^{2}$ University of Michigan (Presented by: Anne M. Suskind, MD, MS)

Objectives: Urodynamic testing is an invasive procedure that can be associated with physical discomfort and emotional distress. The purpose of this study was to identify patients' perceptions of this test according to gender and neurologic status.

Methods: This is a questionnaire-based pilot study completed by patients immediately after undergoing urodynamic testing in the office via a standardized protocol. Participants were asked questions pertaining to their perceptions of the physical and emotional distress related to the study, their urologic and general health history, and their demographics. Results were analyzed overall and were stratified by gender and the presence of neurologic conditions. $\mathrm{P}$ values were not calculated due to sample size limitations.

Results: A total of 108 patients completed the questionnaire and were included in the analysis. The majority of respondents (62.0\%) felt that the exam was neither physically nor emotionally distressing, and $31.0 \%$ felt that the physical distress was worse than the emotional distress, see table. The worst part of the physical discomfort was the placement of the urethral 
catheter (36.8\% in males, $35.1 \%$ in females). Pain associated with placement of the rectal catheter was low in both groups (7.9\% in males and 3.9\% in females). Results stratified by the presence of neurologic conditions followed a similar pattern; however, respondents with neurologic conditions reported no physical discomfort more often (40.7\% compared to $29.7 \%$ in non-neurologic respondents). In terms of emotional distress, most respondents reported no emotional distress associated with the urodynamics study (51.5\%), followed by anxiety (24.2\%), embarrassment (16.2\%), and not understanding what was happening (2.0\%). Results were similar regardless of gender or the presence of a neurologic condition.

Conclusion: Urodynamic studies are well tolerated by men and women both with and without neurologic conditions. Patient perception of physical discomfort tended to be worse than that of emotional discomfort, with placement of the urethral catheter causing the most physical distress.

Clinical Relevance: Urodynamics

\begin{tabular}{|c|c|c|c|c|c|}
\hline & $\begin{array}{l}\text { ONerall } \\
(\mathrm{N}-10 \mathrm{~s})\end{array}$ & $\begin{array}{l}\text { Male } \\
(\mathrm{N}=39)\end{array}$ & $\begin{array}{l}\text { Femsle } \\
\text { (Na60) }\end{array}$ & $\begin{array}{c}\text { Wont:Neuro } \\
(\mathrm{N}=50)\end{array}$ & $\begin{array}{l}\text { Neverc } \\
(\mathrm{N}=2.8\end{array}$ \\
\hline \multicolumn{6}{|l|}{ Worst part of the exam } \\
\hline Physical dichomitort & 31.0 & 316 & 30.4 & 32.9 & 25,9 \\
\hline Enotionsl dikonfort & 50 & 5.3 & 5,4 & 4.1 & 7.4 \\
\hline Eesally bad & 20 & 2.6 & 18 & 1.4 & 3.7 \\
\hline Nether bad & 620 & 60.5 & 62.5 & 61.6 & 63.0 \\
\hline \multicolumn{6}{|l|}{ Fhysical discomfort } \\
\hline Placement of urethral catheter & 35.6 & 36.8 & 35.1 & 39.2 & 25,9 \\
\hline No divconfofort & 327 & 316 & 31.6 & 29.7 & 40.7 \\
\hline Holding a full Elxdder & 10.9 & 7.9 & 14.0 & 10.8 & 11.1 \\
\hline Urinsting & 6.9 & 26 & 10.5 & 6.8 & 7.4 \\
\hline Placement of cathester in rectum & 5.0 & 7.9 & 3.5 & 5.4 & 3.7 \\
\hline Filing blosider with fluid & 3.0 & 2.6 & 1.8 & 2.7 & 3.7 \\
\hline \multicolumn{6}{|l|}{ Emotion d disconfort } \\
\hline None & 51.5 & 47.4 & 54.6 & 500 & 55.6 \\
\hline Asxiety & 24.2 & 26.3 & 23.6 & 25.0 & 22.2 \\
\hline Embarrassment & 16.2 & 184 & 14.6 & 16.7 & 14.8 \\
\hline Not undratanding what wa happening & 2.0 & 26 & 3.6 & 2.8 & 7.4 \\
\hline Fear & 2.0 & 2.6 & 1.8 & 28 & + \\
\hline
\end{tabular}

\section{Podium\#11}

TRENDS IN URODYNAMICS IN U.S. FEMALE MEDICARE BENEFICIARIES, 2000-2010

W. Stuart Reynolds, MD, MPH, Shenghua Ni, PhD, Melissa Kaufman, MD, PhD, David Penson, MD, MPH, Roger Dmochowski, MD

Vanderbilt University, Nashville, TN

(Presented by: W. Stuart Reynolds, MD, MPH)

Introduction: Results of recent clinical trials and recommendations of AUA guidelines have drawn increased attention to the utilization of urodynamics (UDS) in clinical practice. We sought to describe the temporal trends in the use of UDS in female U.S. Medicare beneficiaries.

Material and Methods: Using a 5\% sample of U.S. Medicare utilization records, we identified female beneficiaries who had undergone UDS studies between 2000 and 2010 by the presence of Common Procedural Terminology codes for cystometrogram in claims from the Carrier file. We abstracted data for each patient on age, race, residence, ICD9 diagnoses, dates of service, and provider specialty. We calculated rates per 100,000 beneficiaries with data available from the enrollment files (i.e. Denominator files) and reported the numbers and rates per 100,000 by year.

Results: During this period, 1.4 million female U.S. Medicare beneficiaries underwent UDS, of which $6 \%$ were videourodynamics. $74 \%$ of UDS were associated with a diagnosis of any urinary incontinence, with $50 \%$ specific for stress incontinence.
The annual rates of UDS increased by 29\%, from 422 in 2000 to 543 in 2010 per 100,000. Similar increases were seen across age groups, geographic regions and racial/ethnic groups, with the exception of a disproportional increase in studies in non-white, non-black patients. The rate of UDS performed by gynecologists increased by $144 \%$ over the study period, from 77 to 188, while that of urologists decreased by 3\%, from 326 to 316. In 2010, gynecologists performed $35 \%$ and urologists $58 \%$ of all UDS.

Conclusion: The use of UDS in the female Medicare program increased substantially between 2000 and 2010, with some variation across demographics and marked variation across provider specialty.

Clinical Relevance: Female Urology-including Incontinence

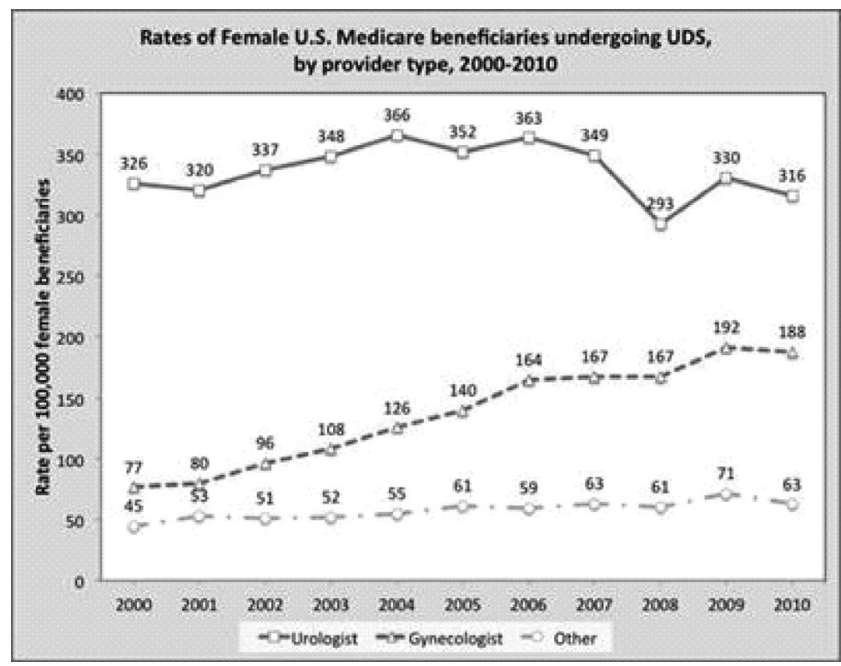

\section{Podium \#12}

\section{COMPARISON OF URODYNAMIC FINDINGS IN NONDIABETIC VERSUS DIABETIC FEMALES}

Rena D. Malik, $M D$, Joshua A. Cohn, $M^{1}$, Charles Chang, $M^{1}$, Sarah L. Garvey ${ }^{2}$, Gregory T. Bales, $\mathbf{M D}^{1}$

and Doreen E. Chung, $\mathrm{MD}^{3}$

${ }^{1}$ University of Chicago Medical Center, Chicago, IL; ${ }^{2}$ Mount Sinai Hospital, Chicago, IL; ${ }^{3}$ University of Chicago Medical Center \& Mount Sinai Hospital, Chicago, IL

(Presented by: Rena D. Malik, MD)

Introduction: Classic diabetic cystopathy has been described as decreased sensation, increased capacity, impaired contractility, and elevated post void residual (PVR). Data are conflicting and few contemporary studies exist regarding urodynamics (UDS) findings in patients with diabetes mellitus (DM). To date no comparative studies exist. Our aim was to compare UDS findings in females with and without DM from a contemporary database.

Methods: A single center database from 2010 to 2013 was searched for females and retrospectively reviewed. Studies were performed according to International Continence Society Standards. For significance, Fisher's exact and Students t tests were performed.

Results: 304 patients underwent UDS between 2010 and 2013. Of those $88(29 \%)$ had DM. Indications for UDS and symptoms at presentation, specifically stress, urge and mixed urinary incontinence, storage symptoms, urinary retention, neurogenic bladder, recurrent urinary tract symptoms, pelvic organ prolapse and pelvic pain, were similar in both groups. Diabetics 
more frequently presented with voiding symptoms (16 vs. 7\%, $p=0.03$ ) and less frequently with nocturia (8\% vs. $20 \%$, $\mathrm{p}=0.01$ ). UDS findings are summarized in Table 1 . Females with DM had significant delay to first sensation, larger bladder capacity (mean $489 \mathrm{~mL}$ vs. $406 \mathrm{~mL}, \mathrm{p}=0.011$ ), and decreased maximum detrusor pressures $(41.7 \mathrm{cmH} 2 \mathrm{O}$ vs. $57.7 \mathrm{cmH} 2 \mathrm{O}$, $\mathrm{p}=0.0006$ ). A larger proportion of females with DM had detrusor underactivity ( $29 \%$ vs. $16 \%, p=0.027)$ and high PVR. There was no difference in presence of detrusor overactivity, stress incontinence, or abdominal straining between groups.

Conclusion: In this contemporary series, women with DM, demonstrated similar presenting complaints to women without DM but had delayed sensation, higher capacity, lower detrusor pressures and increased PVR, consistent with classic diabetic cystopathy. These findings reinforce the importance of UDS in the workup of DM females with lower urinary tract symptoms, particularly prior to initiation of treatment.

\section{Financial Funding: None}

\begin{tabular}{|c|c|c|c|}
\hline & $\begin{array}{l}\text { NonDlabetics } \\
\qquad(n=216)\end{array}$ & $\begin{array}{c}\text { Diabetics } \\
\text { (n=8s) }\end{array}$ & P Value \\
\hline Mean First Sensation $\pm S D(m L)$ & $128 \pm 118$ & $168 \div 169$ & 0.080 \\
\hline Moan Capacity \pm SD(mL) & $406+228$ & $489+306$ & 0.011 \\
\hline Mean $\mathrm{Qmax} 2 \mathrm{sD}(\mathrm{mL} / \mathrm{s})$ & $16 \pm 12$ & $19 \pm 15$ & 0,188 \\
\hline Mean Pdet at Cmax $\pm S D(c m H 2 O)$ & $37+26$ & $28 \pm 23$ & 0.002 \\
\hline Mean Max Pdet $\pm S D(\mathrm{cmHzO})$ & $58+35$ & $42 \pm 30$ & 0.0006 \\
\hline Detrusor Overactivity[\%] & $61(29)$ & $26(31)$ & 0.779 \\
\hline Stress incontinence $[\mathrm{K}$ ) & $106(52)$ & $45(56)$ & 0.600 \\
\hline Bladder Strength(\%) & & & 0.027 \\
\hline Weak & $30(16)$ & $19(29)$ & \\
\hline Strong & $161(84)$ & $46(71)$ & \\
\hline Mean PVR $\pm S D(m L)$ & $28 \pm 59$ & $56 \pm 110$ & 0.004 \\
\hline Abdominal Straining[\%) & $8(43)$ & $42(51)$ & 0.236 \\
\hline
\end{tabular}

\section{Podium \#13}

\section{REEVALUATION OF CUT-OFF VALUES FOR BLADDER OUTLET OBSTRUCTION BASED ON PRESSURE-FLOW STUDIES FROM ANATOMICALLY OBSTRUCTED WOMEN}

Omer Gulpinar, MD, Philippe Zimmern, MD, Alana Christie, Jack C. Hou, MD, Feras Alhalabi, MD, Gary E. Lemack, MD UT Southwestern Medical Center, Dallas, Texas

(Presented by: Philippe Zimmern, MD)

Introduction: To refine the definition of pressure-flow study cut-off values for anatomic female bladder outlet obstruction (BOO) by comparing these urodynamic parameters in women with clinical obstruction due to 3 different obstructive mechanisms.

Methods: Following IRB approval, urodynamic data from 3 groups of women with BOO were reviewed, including Group 1: obstruction after sling placement, Group 2: symptomatic Stage 3-4 anterior vaginal prolapse, Group 3: meatal stricture or distal urethral fibrosis. BOO diagnosis was based on history, presenting symptoms, exam findings, and site of obstruction confirmed on lateral voiding cystogram. Urodynamic testing was done according to prior reported protocol (ref 1), with tracings excluded for abdominal straining (Pabd $>10 \mathrm{~cm} \mathrm{H2O}$ ). The optimal combination of maximal flow rate (Omax) and detrusor pressure at maximal flow rate (PdetQmax) for determining $\mathrm{BOO}$ was calculated using nonparametric receiver operating characteristic (ROC) curves. Box and whisker plots were used for group comparison.

Results: In the past 9 years, 146 non-neurogenic women with clinically-confirmed anatomic BOO (age 61 [41-87]) were studied in comparison to 42 age-comparable controls (age 63 [42-85]). The area under the curve for the ROC was 0.886 for Omax and 0.778 for PdetOmax. For the Omax cut-off of 15, the sensitivity and specificity were 0.78 and 0.82 respectively, while for Pdet Omax cut-off of 25, they were 0.76 and 0.72 respectively (Fig. 1). Age, Omax, and PdetOmax were similar among the three BOO groups, except for lower Omax in Group 3 versus Group 1 (Fig. 2)

Conclusion: This data confirms and reinforces prior data (1) on cut-off values for BOO in women.

Reference:

1. Defreitas et al. Urology 64:675-681, 2004.
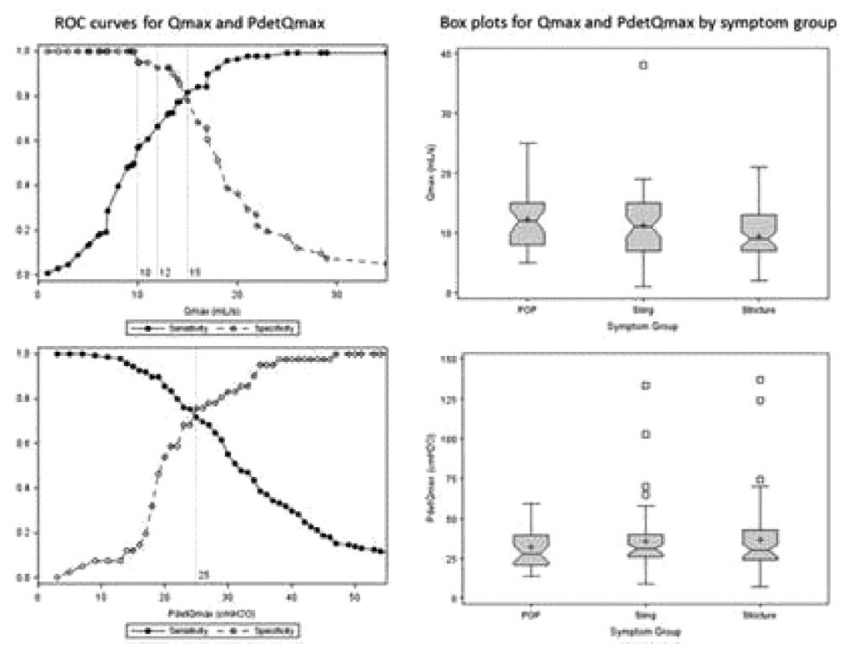

\section{Podium \#14}

PREOPERATIVE VOIDING SYMPTOMS ARE NOT PREDICTIVE OF INCONTINENCE AFTER TRANSURETHRAL RESECTION OF PROSTATE IN POST-RADIATION PATIENTS

Allison Polland, $\mathrm{MD}^{1}$, Emily Vertosick, $\mathrm{MPH}^{2}$

and Jaspreet Sandhu, MD $^{2}$

${ }^{1}$ Mount Sinai Medical Center, New York, New York; ${ }^{2}$ Memorial Sloan-Kettering Cancer Center, New York, New York

(Presented by: Allison Polland, MD)

Introduction and Objectives: Urinary morbidity after radiation therapy for prostate cancer is well known and transurethral resection of the prostate (TURP) is known to be associated with the development of urinary incontinence. The purpose of this study was to evaluate the incidence of post-TURP incontinence in previously radiated patients and determine predictors of urinary continence.

Methods: After IRB approval, 111 patients who underwent brachytherapy or external beam radiotherapy (XRT) with subsequent TURP at a single institution were identified. Patients were excluded if TURP was performed as part of a transurethral resection of a bladder tumor or for a purpose other than relief of lower urinary tract symptoms. A total of 67 patients had one post-radiation TURP, 26 patients had two TURPs and 9 patients had three or more TURPs. Statistical analysis was performed to test for association between postTURP incontinence and pre-TURP urinary symptoms, time from 
radiation therapy to TURP, age at TURP, number of previous TURPs, and decade when radiation therapy was performed. All analyses were adjusted for pre-TURP continence status.

Results: Ninety-six of the total 149 TURP procedures were performed on completely continent patients. Of these 96 procedures, 31 resulted in post-TURP incontinence (32\%). The majority of patients (76\%) received XRT, whether alone or in combination with brachytherapy. Of the 27 patients who were incontinent before their first TURP, 11 patients had resolution of incontinence after the procedure (41\%). Time between radiation and TURP was found to be significantly associated with a change in continence, for every ten years there was a greater than twofold increase in the odds of a change in continence (OR 2.30, 95\% CI 1.11, 4.76, $p=0.025$ ). When using data from all TURPs, there was no evidence of association between pre-TURP urinary symptoms, age at TURP, type of radiation, decade in which radiation was given, or number of previous TURPs, and change in continence after TURP.

Conclusion: A moderate risk of incontinence post-TURP in previously radiated patients was demonstrated in the current study. Time between radiation therapy and TURP was significantly associated with change in continence. In those patients with pre-TURP incontinence, $41 \%$ had resolution of incontinence, however preoperative voiding symptoms were not predictive of postoperative incontinence. These results will aid clinicians in preoperative decision making.

Clinical Relevance: LUTS / Voiding Dysfunction

\section{Podium \#15}

\section{FUNCTIONAL AND ANATOMICAL DIFFERENCES BETWEEN CONTINENT AND INCONTINENT MEN POST RADICAL PROSTATECTOMY ON URODYNAMICS AND 3T MRI}

Anne Cameron, $\mathrm{MD}^{1}$, Anne Suskind, $\mathrm{MD}^{2}$, Charlene Neer, $\mathrm{RN}^{1}$, Hero Hussain, $M D^{1}$, Jeffrey Montgomery, $M^{1}$, Jerilyn Latini, $\mathrm{MD}^{1}$ and John DeLancey, $\mathrm{MD}^{1}$

${ }^{1}$ Ann Arbor, MI; ${ }^{2}$ Anne Arbor, MI

(Presented by: Anne Cameron, MD)

Introduction: There are competing hypotheses about the etiology of post prostatectomy incontinence (PPI). The purpose of this study was to determine the anatomical and functional differences between men with and without PPI.

Methods: Case control study of continent and incontinent men after radical prostatectomy who underwent functional and anatomic studies with urodynamics and 3.0 Tesla MRI. All men were at least 12 months post prostatectomy and none had a history of pelvic radiation or any prior surgery for incontinence.

Results: Baseline demographics, surgical approach and pathology were similar between incontinent (cases) $(n=14)$ and continent (controls) $(n=12)$ men. Among the cases, the average 24 hour pad weight was $400.0 \pm 176.9$ grams with a mean of $2.4 \pm 0.7$ pads per day. Urethral pressure profiles at rest did not significantly differ between groups (92.4 mm cmH2O vs.91.1 $\mathrm{mm}$ cmH2O, $\mathrm{p}=0.95$ ). However, a man's ability to raise his urethral pressure during pelvic muscle contraction was 2.6 times lower among cases compared with than controls (change of 56.3 vs. $147.5 \mathrm{~mm} \mathrm{cmH} 2 \mathrm{O} \mathrm{p}=0.040$ ). Out of the three UPP measurements taken during a Kegel, if only each individual subject's highest value was analyzed the UPP of incontinent patients was half that of dry men $(p=0.031)$. On MRI, in cases, the anatomical urethral sphincter length was 35\% shorter in the sagittal view and 31\% shorter in the coronal view. Also, the bladder neck was 28.9 degrees more funneled (open) among cases. Asymmetry and/or distortion of the sphincter area was noted in $85.7 \%$ of cases and in $16.7 \%$ of controls ( $p=0.001$ ).

Conclusion: In continent men the urethral sphincter is longer, there is less distortion of the sphincteric area and the bladder neck is less funneled compared to incontinent men on MRI. Urodynamically, during a Kegel maneuver, continent men were much better at augmenting urethral pressures than their wet counterparts. All of these findings suggest that the sphincter in men with PPI is both diminutive and poorly functional. A new finding is the importance of a more widely open bladder neck contributing to incontinence that warrants further investigation.

Funding: MICHR UL1RR024986, NIH/NIDDK T32 DK07782, SCOR HD P50 44406

Clinical Relevance: Urodynamics
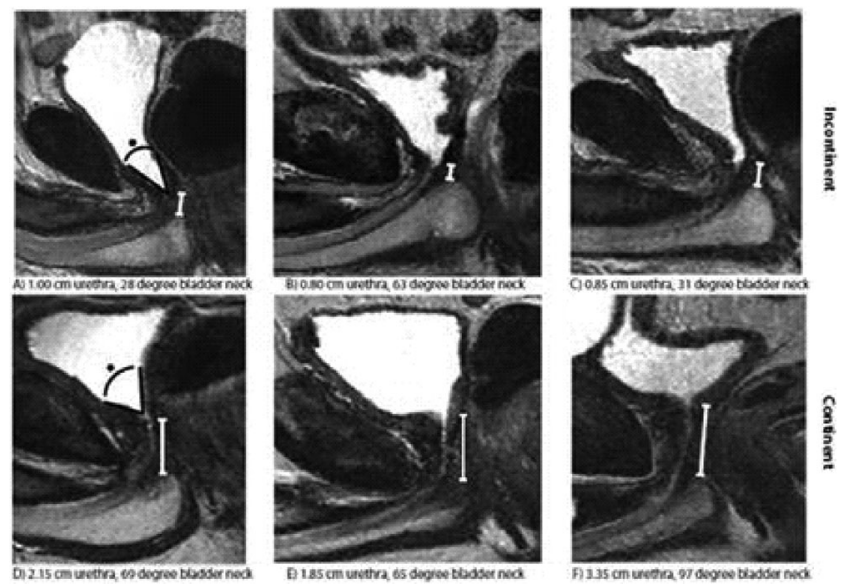

Podium \#16

INCREASING AGE, OBESITY, AND DIABETES INDEPENDENTLY ASSOCIATED WITH URINARY INCONTINENCE AFTER ROBOTIC PROSTATECTOMY IN HISPANIC MEN

Daniel Hoffman, MD, Ricardo Sánchez-Ortiz, MD

University of Puerto Rico, San Juan, $P R$

(Presented by: Daniel Hoffman, MD)

Introduction and Objectives: According to the American Diabetes Association, Puerto Ricans have the highest rate of diabetes mellitus (DM) of any ethnicity in the US (13.8\% vs. $7.1 \%$ of nonHispanic Caucasians). DM has been associated with a higher risk of post prostatectomy incontinence. We present the initial report of the impact of DM on continence after robotic prostatectomy (RP) in Puerto Rican men.

Methods: A prospective database was created for 453 consecutive prostate cancer patients who underwent RP by a single surgeon. The cohort consisted of 300 men with followup $\geq 1$ year. Clinical variables were correlated with continence one year after surgery, defined as zero pads per day after 12 months. Multivariate analysis was performed with SPSS.

Results: Obtained: After a median followup of 25.2 mo., 9.7\% (29/300) of men exhibited stress incontinence (SUI). Fifteen (45/300) percent of all patients had type II DM and 28\% (83/300) were obese or greater. The 3 variables independently predictive of SUI in multivariate analysis were age (mean: 60.3 in incontinent men vs. 57.4 yrs, $p<0.04$ ) (Odds ratio $(O R): 3.25$, $95 \%$ Confidence Intervals (CI): 1.45 to 7.31 ), BMI (28.9 vs. 27.5 , $\mathrm{p}<0.05)$ (OR:2.09, 95\% CI:1.02 to 4.73), and a history of DM (31\% of men with SUI had DM vs. $12.3 \%, p<0.03$ ) (OR:2.59, $95 \%$ 
CI:1.07 to 6.31). The risk of SUI increased linearly both with BMI: (BMI < 30: 8.8\%, BMI 30 to 34.5: 12.7\%; BMI 35 to 39.9: 22.2\%, and $\mathrm{BMI} \geq 40: 33.3 \%$ ) and age: (age $\leq 50: 2.3 \%$, age 51 to $60: 6.8 \%$, age 61 to 70 : $16 \%$, and age $\geq 71$ : $25 \%$ ) ( $<<0.05)$. Diabetic patients exhibited a $20 \%$ risk of SUI compared with $7.8 \%$ in nondiabetics $(p<0.03)$, even in patients with a BMI $\leq 25(42.9 \%$ with $\mathrm{DM}$ vs. $4.1 \%, \mathrm{p}<0.003)$. No other variables adversely affected SUI including PSA, prostate weight, OR time, EBL, anastomotic leak, nerve sparing status, history of hypertension, or smoking.

Conclusion: Puerto Rican patients undergoing RP have double the incidence of DM than the US population and a high rate of obesity. While Puerto Rican men who underwent RP who were not diabetic, had a BMI $<30$ and were younger than 61 years had a SUI rate of $3.4 \%$, this increased to $14.5 \%$ with a $\mathrm{BMI} \geq 30,16.3 \%$ when older than 60 years, and beyond $20 \%$ with DM even in the absence of obesity. These are important data to discuss with patients in order to establish realistic expectations.

Funding: none

Clinical Relevance: Male Incontinence

\section{Podium \#17}

LONG-TERM OUTCOMES FOR ARTIFCIAL URINARY SPHINCTER REIMPLANTATION FOLLOWING PRIOR DEVICE EXPLANTATION FOR EROSION OR INFECTION

Brian Linder, MD, Daniel Elliott, $M D$

Mayo Clinic, Rochester, MN

(Presented by: Brian Linder, MD)

Objectives: To evaluate the clinical outcomes of patients managed with artificial urinary sphincter (AUS) reimplantation following AUS explantation for erosion or infection.

Methods: We identified 704 consecutive AUS implantation procedures performed at Mayo Clinic from 1998 to 2012. Of these, 497 were primary implantations (71\%), 138 (20\%) were revision surgeries for device malfunction and 69 patients $(10 \%)$ had undergone at least one prior AUS explantation secondary to urethral erosion and/or device infection (i.e. salvage implantation). Patient follow-up was obtained through office examination, written or telephone correspondence.

Results: Patients undergoing salvage AUS reimplantation had a median age of 78 (IOR 72, 80), with a median follow-up of 34 months (IOR 5, 61). AUS reimplantation occurred at a median of 9 months (IOR 6, 13) after explantation. Of the 69 patients undergoing salvage placement, 36 (52\%) had undergone multiple (range 2-5) prior reimplantation procedures. Patients undergoing reimplantation after erosion or infection were more likely to require repeat explantation compared to those undergoing primary implantation (13/69 [19\%] versus $32 / 497$ [6.4\%], $p=0.002)$. However, when evaluating all repeat procedures (explantation and revision), no significant difference in the 5-year device survival rate was seen among AUS reimplantations after erosion or infection when compared to primary implantations (68\% versus $76 \%$; $\mathrm{p}=0.38$ ).

Conclusion: Our findings suggest that AUS reimplantation following explantation for urethral erosion and/or device infection is associated with an increased rate of recurrent erosion/infection requiring repeat explantation. However, in appropriately selected and counseled patients, clinically acceptable long-term device survival can be obtained.

Clinical Relevance: Male Incontinence

\section{Podium \#18}

THE IMPACT OF LENGTH OF URETERAL RESECTION ON BENIGN URETERO-INTESTINAL STRICTURE RATE IN ILEAL CONDUIT OR ILEAL NEOBLADDER URINARY DIVERSION FOLLOWING CYSTECTOMY

Joshua Cohn, $M D^{1}$, Kyle Richards, $M^{1}$, Michael Large, $M^{2}$, Gregory Bales, $M D^{1}$, Norm Smith, $M D^{1}$ and

Gary Steinberg, $M^{1}$

${ }^{1}$ University of Chicago, Chicago, IL; ${ }^{2}$ Urology of Indiana, Indianapolis, IN

(Presented by: Joshua Cohn, MD)

Introduction and Objectives: The incidence of benign ureterointestinal anastomotic (UIA) stricture in ileal-segment urinary diversion (UD) is reported at 1-13\%. Resection of the distal ureter is felt to decrease the risk of stricture. We aimed to assess the impact of the length of ureter resected on UIA stricture rate. Methods: We identified 575 consecutive patients who underwent cystectomy and UD from January 2007 to August 2012. Patients who died within 30 days of surgery, had missing ureteral resection data or underwent Indiana pouch UD were excluded. Length of ureter resected was defined as ureter in cystectomy specimen plus distal ureter submitted to pathology. Primary endpoint was time to UIA stricture, assessed by Cox proportional hazards analysis to account for differential follow-up.

Results: obtained: 463 patients met inclusion criteria. Median follow-up was 459 days (IOR 211-927). There were 425 right ureters and 439 left ureters at risk for stricture in the cohort; median time to stricture was 235 (134-352) and 232 days (132351 ) on the right and left, respectively. Overall stricture rate per ureter was $5.9 \%$ on right vs. $10.0 \%$ on left $(p=0.03)$. There was no difference in age, gender, BMI, Charlson Comorbidity Index, pathology, or chemotherapy rate between patients with and without UIA stricture. $62 \%$ of patients underwent ileal conduit and $38 \%$ ileal neobladder UD. There was no difference in diversion type, running vs. interrupted anastomosis or total ureter resected between patients with and without UIA stricture. On univariate analysis, urine leak $(p=0.001)$, abscess $(p=0.012)$ and 30-day Clavien complication $\geq$ III significantly predicted for left-sided UIA stricture whereas only urine leak $(p=0.006)$ significantly predicted for right-sided UIA stricture. On multivariate analysis adjusted for age, gender, anastomosis technique, urine leak, 30-day Clavien complication $\geq$ III and length of ureter resected, only Clavien complication $\geq$ III (HR 2.11, 1.01-4.40) and urine leak (HR 3.37, 1.08-10.46) significantly predicted for left- and right-sided stricture, respectively. Length of ureter resected did not predict for UIA stricture on either side.

Conclusion: The etiology of benign UIA stricture following ileal UD is likely multifactorial. Our data suggest that a complicated postoperative course and urine leak are risk factors for UIA stricture. Length of distal ureter resected may not significantly impact stricture rate.

Clinical Relevance: Reconstruction

\section{Podium \#19}

FIFTEEN YEARS EXPERIENCE WITH THE ANTERIOR VAGINAL WALL SUSPENSION PROCEDURE, A NATIVE TISSUE VAGINAL REPAIR FOR STRESS URINARY INCONTINENCE WITH EARLY STAGE ANTERIOR COMPARTMENT PROLAPSE

Philippe Zimmern, $\mathrm{MD}^{1}$, Alana Christie ${ }^{1}$, Xian-Jin Xie ${ }^{2}$, Chasta Bacsu, $\mathrm{MD}^{3}$, Dominic Lee, $\mathrm{MD}^{1}$, Benjamin Dillon, $\mathrm{MD}^{1}$, Jack Hou, $\mathrm{MD}^{1}$ and Feras Alhalabi, $\mathrm{MD}^{1}$ 
${ }^{1}$ UT Southwestern Medical Center, Dallas, Texas; ${ }^{2} U T$ Southwestern Medical Center, Dallas, Texas; ${ }^{3}$ UT Southwestern Medical Center, Dallas, TX

(Presented by: Philippe Zimmern, MD)

Introduction and Objectives: To report on the mid- and longterm outcome for the anterior vaginal wall suspension (AVWS) procedure (1) for stress urinary incontinence (SUI) with early stage anterior compartment prolapse.

Methods: Following IRB approval, the long-term prospective Access database of non-neurogenic women who underwent AVWS for SUI, with a minimum 1 year follow-up, was reviewed in women with complete pre and post-operative records. Preoperative evaluation included detailed history, validated questionnaires, (UDI-6, OoL), physical examination, and standing lateral voiding cystourethrogram (VCUG) with rest (UAR)/ strain (UAS) views to evaluate urethral hypermobility and degree of cystocele (Lateral height). Follow-up included examination, questionnaires, and one VCUG around 6-12 months post-operatively. Success was measured by KaplanMeier curves using time to reoperation for incontinence. Mixed-effects model least square means were used for baseline versus post-AVWS mean score comparison and for follow-up period mean score comparison (2).

RESULTS: Between 1996 and 2011, 247 women met inclusion criteria. Mean \pm Sd were: age 61.9 (11.2), BMI 27.2 (5.6), parity 2.4 (1.3). 101 (39\%) patients underwent AVWS alone and 60 (23\%) had prior anti-incontinence procedures. Aa and Ba points, questionnaire results, and OoL consistently improved postoperatively, and remained stable over time (Table 1). VCUG findings also improved for urethral support and cystocele reduction Additional therapy with sling (6) or injectable agents (13) was required in 19 (7\%) women (Figure 1).

CONCLUSION: The AVWS procedure can durably correct SUI by restoration of anatomical support to the bladder neck and bladder base.

Reference: 1. 37. Wilson TS, Zimmern PE: Anterior Vaginal Wall Suspension, Female Urology, Urogynecology, and Voiding Dysfunction, Vasavada, Appell, Sand, and Raz, Section I, Chapter 17, 283-290, Marcel Dekker, 2005

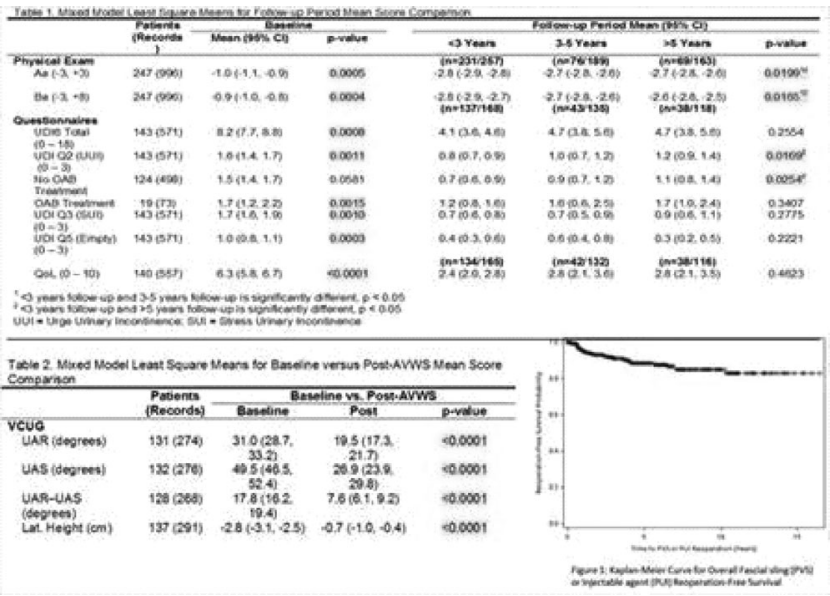

\section{Podium \#20}

\section{ABDOMINAL MESH SACROCOLPOPEXY WITHOUT PROMONTORY FIXATION-THE PERITONEOCOLPOPEXY TECHNIQUE}

Dominic Lee, $\mathrm{MD}^{1}$ and Philippe Zimmern, $\mathrm{MD}^{2}$
${ }^{1}$ UT Southwestern Medical Center, Dallas, TX; ${ }^{2}$ UT Southwestern Medical Center, Dallas, Texas

(Presented by: Philippe Zimmern, MD)

Introduction and Objectives: The anchoring of mesh at the sacral promontory during abdominal sacrocolpopexy (SCP) for apical prolapse can lead to devastating complications including discitis. We present our experience and early outcomes utilizing a new technique for mesh anchorage.

Methods: A prospective review of consecutive patients with symptomatic apical pelvic organ prolapse whom underwent an open mesh peritoneocolpopexy (PCP) with V-lock sutures (Figure 1) from an IRB approved sacrocolpopexy database was performed. Data included demographic characteristics, prior anti-incontinence/genitourinary surgery, complications, validated questionnaires, $\mathrm{POP}-\mathrm{O}$ examination and associated outcomes.

Results: Ten patients underwent PCP from 1/2010 to 12/2012 with at least 6 months follow up. Nine had previous hysterectomy and 7 previous surgery for pelvic organ prolapse (POP) and/or stress incontinence. Mean age and median follow up were 65 years (54-82) and 19 months (6-36) respectively. Mean preoperative C-point was $-2.7(+1$ to -4$)$ compared to $-9.7(-12$ to -10$)$ postoperatively $(p<0.001)$. Mean operative time, blood loss and postoperative length of stay were 219 minutes (160-270), $61 \mathrm{ml}(20-200)$ and 2.2 days (1-5) respectively. No patients developed recurrent vault prolapse or required reoperations. Functional outcome improvement was noted with score reduction for OoL of 4.1 to 1.9 ( $p<0.12$ ), UDI-6 of 6.4 to $3(p=0.09)$, and IIO-7 of 7 to $0.7(p=0.4)$ (Table 1 ).

Conclusion: PCP performed reliably to correct symptomatic apical POP with the use of strong unidirectional and delayed absorbable sutures to integrate the mesh to the peritoneum and surrounding fat around the vaginal apex.

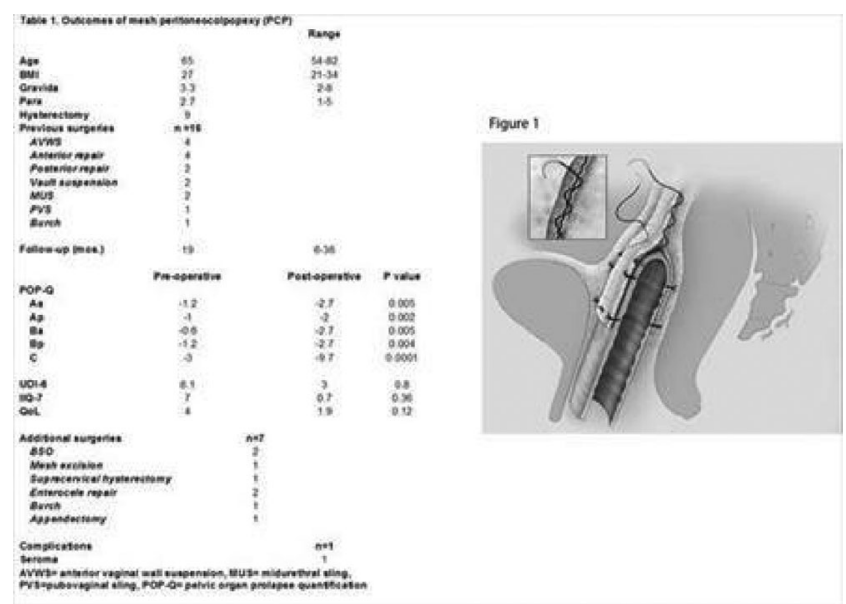

\section{Podium \#21}

NATURAL HISTORY OF PELVIC ORGAN PROLAPSE (POP) IN SYMPTOMATIC PATIENTS ACTIVELY SEEKING TREATMENT

Javier Pizarro-Berdichevsky, $\mathrm{MD}^{1},{ }^{2},{ }^{3}$, Alejandro Pattillo, $\mathrm{MD}^{4},{ }^{5}$, Marco Arellano, $\mathrm{MD}^{4}$, Simone Pohlhammer, $\mathbf{M D}^{4}$ and Howard B Goldman, MD $^{6}$

${ }^{1}$ Hosp. Dr. Sotero del Rio; ${ }^{2}$ Division de Obstetricia y Ginecologia, Facultad de Medicina, Pontificia Univ. Catolica de Chile;

${ }^{3}$ Glickman Urology Institute, Cleveland Clinic, Clevaland, Ohio;

${ }^{4}$ Urogynecology Unit, H. Dr. Sotero del Rio, Santiago, Chile; 
${ }^{5}$ Division Obstetricia y Ginecologia, Pontificia Universidad Catolica de Chile; ${ }^{6}$ Glickman Urologic Institute, Cleveland Clinic, Cleveland, Ohio

(Presented by: Javier Pizarro-Berdichevsky, MD)

Introduction: There is minimal data on progression of POP in symptomatic women. At our institution, many patients may wait months or years for surgery. We describe prospectively the evolution of POP in these patients and identify risk factors associated with progression of POP.

Materials and Methods: A prospective cohort of patients of the urogynecology unit of Dr. Sotero del Rio public hospital in Santiago, Chile was evaluated between July 2008 and June 2013. Women with symptomatic (symp) POP having 2 or more POP-Q examination in follow-up (FU) (still awaiting surgery) or prior to surgery were included. They were compared to similar asymptomatic (asym) women. Demographic data, prolapse symptoms, and initial and last FU POP-O were analyzed. Changes of $\geq \pm 2 \mathrm{~cm}$ in the POP-O measurements of either points $\mathrm{Ba}, \mathrm{Bp}$ or $\mathrm{C}$ between initial and $\mathrm{FU}$ were defined as clinically significant. Isolated analysis by compartment was performed.

Results: 423 patients met the inclusion criteria. 392 were symp and 31 asym. Median FU was (symp/asym): 9/12 months. POPO staging percentages at admittance (symp/asym): 0-I 0.1/6.4\%, II $36.2 / 90.3 \%$, III-IV 62.7/3.2\% POPO Changes (symp/asym): $47.5 / 19.3 \%$ progressed, $8.7 / 6.4 \%$ regressed and $43.8 / 74.3 \%$ did not change. In the symp group, same compartment progression was observed in $21.7 \%, 7.1 \%$ and $23 \%$ in the $\mathrm{Ba}, \mathrm{Bp}$ and $\mathrm{C}$ points respectively. When the leading edge was the anterior compartment, $48 \%$ of the progressions were related to only the $C$ point. Analysis of the survival curves for progression demonstrated: in symp patients the median time (p50) to progression was at 10 months FU (CI 95\% 9.3-10.6) (see graph). Analysis by leading edge at admittance demonstrated significant differences for time to progression between Bp (p50 at 19 months FU 95\% CI 10.8-27.2) and Ba (p50 at 9 months FU 95\% CI 8.1-9.8) as well as $\mathrm{Bp}$ and C (p50 at 9 months FU 95\% CI 7.9-10.1).

Conclusion: The natural evolution of symp POP is progression in more than $45 \%$ of patients. Regression of POP occurs less often (8.7\%). $48 \%$ of patients with $\mathrm{Ba}$ POP had significant progression of the apex ( $\mathrm{C}$ point). The posterior compartment less commonly progresses. Progression is significantly more common in symp patients than in asym patients.

Clinical Relevance: Pelvic Organ Prolapse

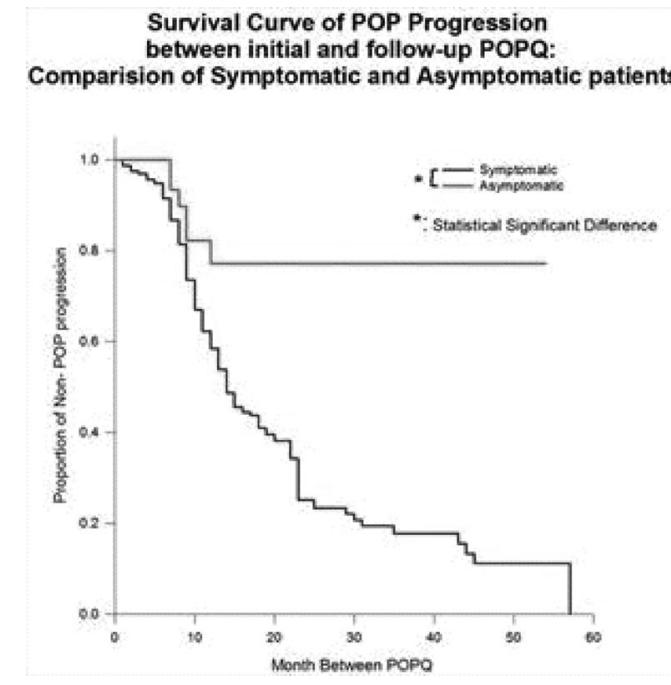

\section{Podium \#22}

URODYNAMIC FINDINGS IN WOMEN WITH HYDRONEPHROSIS DUE TO ADVANCED PELVIC ORGAN PROLAPSE

Christina Dancz, $\mathrm{MD}^{1}$, Daphne Walker, $\mathrm{MD}^{2}$, Diane Thomas, $\mathbf{M D}^{2}$ and Begum Ozel, $\mathbf{M D}^{2}$ ${ }^{1}$ University of Southern California; ${ }^{2}$ University of Southern California, Los Angeles, CA

(Presented by: Christina Dancz, MD)

Introduction: Advanced pelvic organ prolapse (POP) may be a cause of obstructive uropathy causing hydronephrosis.

Objectives: 1) To describe the prevalence of hydronephrosis in advanced POP 2) To describe clinical and urodynamic parameters associated with hydronephrosis.

Methods: This is a prospective observational study of the prevalence of hydronephrosis in advanced POP. Women presenting to LAC + USC Medical Center for urogynecologic care between October 2010 and April 2013 with a POP-O exam of at least +1 for points $C$, Aa or Ba were enrolled and screened for hydronephrosis. Clinical and urodynamic factors among women with and without hydronephrosis were compared. The University of Southern California IRB approved this protocol. This project was funded by a grant from the American Institute of Ultrasound in Medicine.

Results: 180 participants were enrolled. 55 women had some degree of hydronephrosis, for a prevalence of $30.6 \%$ (24.3$37.6 \%)$. Mean age was $57.9(+/-9.0)$ years and mean BMI was $29.2 \mathrm{~kg} / \mathrm{m} 2$ (+/ - 4.6). 80.6\% of participants were postmenopausal. There was no difference between groups among women with and without hydronephrosis by age, race, BMI, parity, menopausal status, hypertension, or creatinine level. A history of diabetes was significantly associated with hydronephrosis ( $8 \%$ vs. $21.8 \%, p=0.009$ ), as was the degree of anterior and apical, but not posterior POP (table). On multichannel urodynamics, participants with hydronephrosis had higher mean post void residual volumes (PVR) (64.8mL vs. $38.5 \mathrm{~mL}, \mathrm{p}=0.007$ ), lower mean volume at first leak (199.6mL vs. $280.8 \mathrm{~mL}, \mathrm{p}=0.006)$ and higher mean maximum cystometric capacity $(525.2 \mathrm{~mL}$ vs. $476.7 \mathrm{~mL}, \mathrm{p}=0.02)$ when compared to participants without hydronephrosis. There was no difference between groups in maximum flow rate, detrusor pressure at maximum flow, maximum detrusor pressure, or valsalva leak point pressure ( $p>0.05)$.

Conclusion: The prevalence of hydronephrosis in women with advanced pelvic organ prolapse is $30.6 \%$. Clinical factors significantly associated with hydronephrosis include degree of anterior or apical POP and diabetes. Urodynamic factors associated with hydronephrosis include elevated PVRs, larger cystometric capacity and lower volume at first leak.

Clinical Relevance: Urodynamics

\begin{tabular}{|l|l|l|l|l|}
\hline & $\begin{array}{l}\text { POP-Q } \\
\text { Measure }\end{array}$ & $\begin{array}{l}\text { Without } \\
\text { Hydronephrosis }\end{array}$ & $\begin{array}{l}\text { With } \\
\text { Hydronephrosis }\end{array}$ & P \\
\hline Apical & C & $1(-6$ to 8.5$)(n=122)$ & $\begin{array}{l}4.5(-5 \text { to } 11) \\
(n=54)\end{array}$ & $<0.001$ \\
\cline { 2 - 6 } & D & $-4(-7$ to 4$)(n=120)$ & $\begin{array}{l}-1(-7 \text { to } 11) \\
(n=53)\end{array}$ & $<0.001$ \\
\hline Anterior & Aa & $2(0$ to 4$)(n=125)$ & $3(-1$ to 3$)(n=55)$ & $<0.001$ \\
\cline { 2 - 6 } & Ba & $2(0$ to 8$)(n=125)$ & $5(0$ to 11$)(n=55)$ & $<0.001$ \\
\hline Posterior & Ap & $3(-3$ to 3$)(n=122)$ & $0(-2$ to 3$)(n=52)$ & 0.13 \\
\cline { 2 - 6 } & Bp & $0(-3$ to 7$)(n=122)$ & $0(-2$ to 9$)(n=52)$ & 0.20 \\
\hline
\end{tabular}

\section{Podium \#23}

THE USE OF DEFECOGRAPHY VERSUS ANAL MANOMETRY TO DIAGNOSE DISORDERS OF THE FEMALE PELVIC FLOOR 
Alexandriah N. Alas, $\mathrm{MD}^{1}$, Benjamin Basseri, $\mathrm{MD}^{2}$, John F. Bray, $\mathrm{MD}^{3}$, Sharon Kim, $\mathrm{MD}^{4}$, Andres F. Ardila Hani BS ${ }^{4}$, Avetis Azizyan, $\mathrm{MD}, \mathrm{PhD}^{3}$, Mark Pimentel, $\mathrm{MD}^{4}$, Edy Soffer, $\mathrm{MD}, \mathrm{MPH}^{5}$, Christopher Chang, $\mathrm{MD}, \mathrm{PhD}^{4}$, Jennifer T. Anger, $M D, M P H^{6}$ and Jeffrey L. Conklin, $M^{4}$

${ }^{1}$ Cleveland Clinic Florida, Weston, FL; ${ }^{2}$ University of California, Los Angeles (UCLA) Medical Center, Los Angeles, CA; ${ }^{3}$ Radiology, Cedars-Sinai Medical Center, Los Angeles, CA; ${ }^{4}$ Division of Gastroenterology, Cedars-Sinai Medical Center, Los Angeles, CA; ${ }^{5}$ Division of Gastroenterology, University of Southern California, Los Angeles, CA; ${ }^{6}$ Department of Surgery, Division of Urology, Cedars-Sinai Medical Center, Los Angeles, CA

(Presented by: Alexandriah N. Alas, MD)

Introduction and Objectives: High-resolution anorectal manometry (HR-ARM) is considered the gold standard in evaluating the internal and external anal sphincter for fecal incontinence. Currently, defecography is mostly used as an adjunctive tool to compliment clinical assessment and anorectal manometry. The main study objective was to assess the agreement between HR-ARM and defecography.

Methods: This was a retrospective search for women who had both HR-ARM and defecography. Data was collected on presenting symptoms, presence of enterocele, rectocele, intussusception, pelvic floor spasm, and fecal incontinence. We then further assessed the diagnostic yield of both techniques and determined if the use of both studies provided information not obtained with a single test.

Results: There were 141 women identified who had both defecography and HR-ARM. Indications for the procedures (multiple in many subjects) included constipation (70\%), incomplete evacuation (61\%), and fecal incontinence (30\%). Constipation was the most common complaint found for rectocele (78.1\%), intussusception (61.5\%), and pelvic floor spasm (73.3\%). The most common complaint for enterocele was incomplete evacuation or a sensation of fullness (78.9\%). Enterocele, rectocele, intussusception (or rectal prolapse), and descending perineum syndrome (DPS) were detected on defecography in $27.1 \%, 45.7 \%, 27.9 \%$, and $45 \%$ respectively. Pelvic floor spasm and rectal hypersensitivity were detected more frequently on HR-ARM (43.1 vs $24.2 \%$; $p<0.0007$ and $53.2 \%$ vs $22 \% ; p<0.0001)$, respectively. Overall agreement between the two tests was $77.1 \%$, and performing both procedures added value in $97.2 \%$ of cases.

Conclusion: This is the largest examination, to our knowledge, investigating the correlation between anorectal manometry (or HR-ARM) and defecography in women with defecatory dysfunction. Defecography elucidated significant anatomical abnormalities (enterocele, rectocele, intussusception) that were undetectable with HR-ARM. There was overall agreement with clinical impressions between the two diagnostic tests. Defecography and HR-ARM together provide significantly more diagnostic information than either test alone.

Clinical Relevance: Pelvic Organ Prolapse

\section{Podium \#24}

THE OUALITY OF CARE PROVIDED TO WOMEN WITH PELVIC ORGAN PROLAPSE: AN INFRASTRUCTURE FOR OUALITY ASSESSMENT

Alexandriah N. Alas, $\mathrm{MD}^{1}$, Catherine Bresee, $\mathrm{MS}^{2}$, Karyn S. Eilber, $\mathrm{MD}^{3}$, Karen Toubi BS ${ }^{3}$, Rezoana Rashid $\mathrm{BS}^{3}$, Carol P. Roth, RN, $\mathrm{MPH}^{4}$, Paul G. Shekelle, MD, $\mathrm{PhD}^{4,5}$, Neil S. Wenger, $M^{6}$ and Jennifer T. Anger, $M D, M^{\prime} H^{3}$
${ }^{1}$ Cleveland Clinic Florida, Weston, FL; ${ }^{2}$ Samuel Oschin Cancer Institute, Cedars-Sinai Medical Center, Los Angeles, CA; ${ }^{3}$ Department of Surgery, Division of Urology, Cedars-Sinai Medical Center, Los Angeles, CA; ${ }^{4}$ Southern California EvidenceBased Practice Center RAND Corporation, Los Angeles, CA; ${ }^{5}$ Veterans' Affairs Greater West Los Angeles Medical Center, Los Angeles, CA; ${ }^{6}$ David Geffen School of Medicine, University of California - Los Angeles, Los Angeles, CA

(Presented by: Alexandriah N. Alas, MD)

Introduction and Objectives: Our aim was to assess the feasibility of recently developed quality indicators (OIs) for pelvic organ prolapse (POP) and, identify deficits in patient care. Methods: A panel ranked 14 OIs based on RAND Appropriateness Method assessing screening and diagnosis, treatment with pessaries, and surgical management of POP. Retrospective chart abstraction was performed using ICD-9 codes.

Results: Obtained: Total of 283 patients. Ninety-eight percent of patients with new complaint of vaginal bulge had a pelvic exam. POP was described but not staged in $6 \%$, and not documented at all in $25.1 \%$. Pessary was discussed in $43 \%$ of eligible patients. Among those managed with pessaries, $98 \%$ had vaginal exams every 6 months. Among women who underwent surgery, only $49 \%$ had complete pre-operative staging of the anterior, posterior, and apical compartments. Only 20\% of women undergoing apical surgery had documentation of counseling about different surgical approaches, but $71 \%$ had counseling about the risk of post-operative stress incontinence. Only $14.5 \%$ of patients had documented counseling regarding risks of mesh. Only $48 \%$ of hysterectomies for POP had a concomitant vault suspension. Intra-operative cystoscopy was performed in $86 \%$ undergoing anterior and/or apical surgery. Within three months of surgery, $89 \%$ of patients had a post-operative pelvic exam. Only $37 \%$ who received mesh had documented follow-up at one year.

Conclusion: We demonstrated the 14 OIs are feasible in that they are identifiable in medical records and can be used to measure quality through retrospective chart abstraction. Overall, care to patients was adequate, but deficits in care were identified.

Funding: NIDDK (K23 DK080227-01, JTA)

Clinical Relevance: Pelvic Organ Prolapse

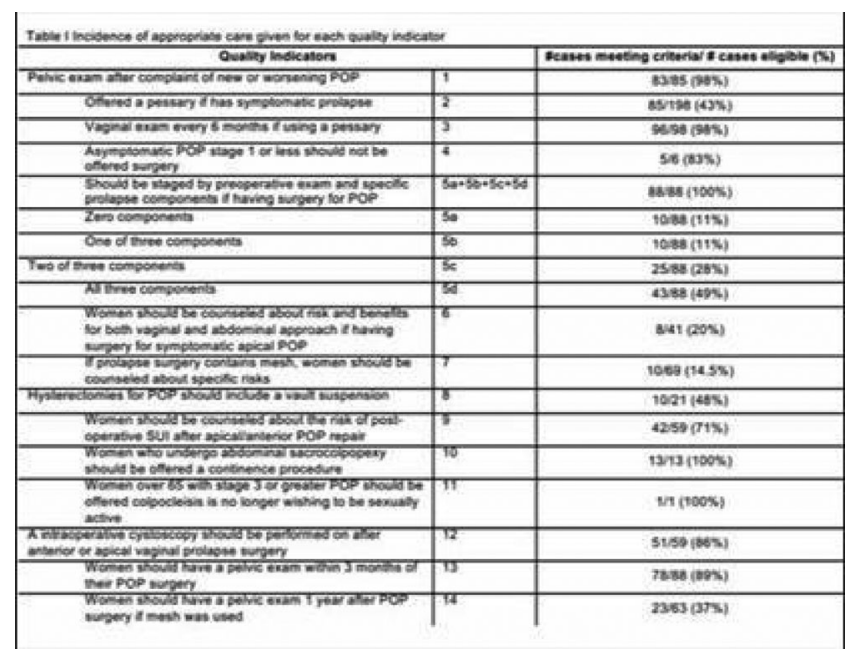

Podium \#25

UTEROVAGINAL PROLAPSE: TRADITIONAL RECONSTRUCTIVE SURGERY OUTCOMES 
Luis Espaillat-Rijo, $\mathrm{MD}^{1}$, Aparna Hegde, $\mathrm{MD}^{2}$, Asra Jawed, $\mathrm{MD}^{2}$ and G. Willy Davila, $\mathbf{M D}^{2}$

${ }^{1}$ Cleveland Clinic Florida; ${ }^{2}$ Cleveland Clinic Florida, Weston, FL (Presented by: Luis Espaillat-Rijo, MD)

Objective: In an era when the use of mesh has come under scrutiny for its safety profile, we evaluated the anatomical and functional outcomes of native tissue repair by evaluating the total vaginal hysterectomy (TVH) with a McCall culdoplasty and associated vaginal repairs in women with advanced uterovaginal prolapse.

Methods: A retrospective database review of patients who underwent a TVH with culdoplasty from January 2001 and August 2012 was conducted after obtaining IRB approval. Only charts with a minimum of 1 year of follow-up data were included for review. Pre and post-operative anatomic measurements (Baden Walker Halfway system and POP-O), self reported satisfaction using the validated Improvement Satisfaction Scale (ISS), sexual function and complications were extracted and analyzed. Prolapse recurrence was defined as grade 2 or more.

Results: A total of 622 patients were identified, with 242 (38.9\%) patients having at least 1 year of follow-up data. The median (interquartile range) follow-up time was 103 (111) weeks. 240 (99.2\%) patients had adequate anatomical support, and $2(0.8 \%)$ patients suffered recurrence. 183 (75.62\%) patients and 229 (94.63\%) patients had concomitant anterior and posterior vaginal wall repairs, respectively. Anatomic results are listed in Table 1. Kaplan-Meier analysis was performed to account for variable weeks of follow-up (figure 1). 218 (90.8\%) patients reported being improved after surgery. De novo dyspareunia was reported by 14 (11.3\%) patients. Vaginal discharge was reported by 10 (4\%) patients and granulation tissue was reported in $1(0.4 \%)$ patient. No suture infections or erosions were noted.

Conclusion: A traditional surgical approach to uterovaginal prolapse is very effective anatomically and functionally with minimal healing-related complications.

Clinical Relevance: Reconstruction

Tuble 1: hre and poet POPQ nilues

\begin{tabular}{|c|c|c|c|}
\hline & 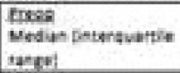 & 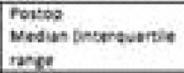 & Fubs \\
\hline Bil & काष्य & माम & नमूक \\
\hline 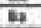 & इका & .911 & 40,051 \\
\hline $\bar{c}$ & -161 & $+4 \mid$ & E.0.001 \\
\hline I & TCH & गयI & सक्6ि \\
\hline B & 3 का & $4 \mid 41$ & 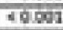 \\
\hline$T \mathrm{~T}$ & प.Sम & पमय & $40.0 \%$ \\
\hline At & पक्ष & पारो & कीका \\
\hline in & -107 & .III & 80,000 \\
\hline
\end{tabular}

Vigure 1: Kuplan-Mcias andigh

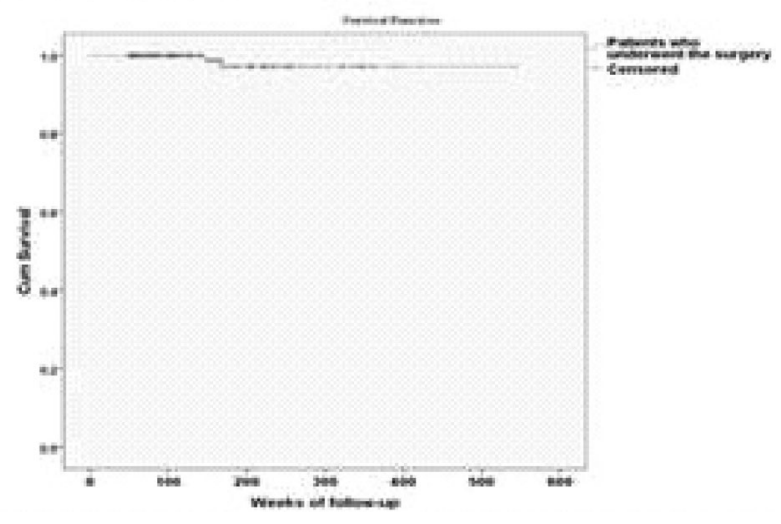

\section{Podium \#26}

\section{IMPROVEMENT IN HYDRONEPHROSIS AFTER PESSARY} PLACEMENT: A SELF-SELECTED INTERVENTIONAL TRIAL

Christina Dancz, $\mathrm{MD}^{1}$, Daphne Walker, $\mathrm{MD}^{2}$, Diane Thomas, $\mathrm{MD}^{2}$, Nuzhath Hussain, $\mathbf{M D}^{2}$ and Begum Ozel, $\mathbf{M D}^{2}$

${ }^{1}$ University of Southern California; ${ }^{2}$ University of Southern California, Los Angeles, CA

(Presented by: Christina Dancz, MD)

Objective: To determine the efficacy of pessary as a treatment for hydronephrosis due to pelvic organ prolapse (POP).

Methods: This is a subset analysis of a prospective study on the prevalence of hydronephrosis in women with advanced POP. Women presenting to LAC + USC Medical Center for care between October 2010 and April 2013 with POP at least $1 \mathrm{~cm}$ outside of the body were enrolled and screened for hydronephrosis. All subjects were offered expectant management, pessary or surgery. Subjects with hydronephrosis were included for analysis. Basic demographics and degree of subsequent hydronephrosis were recorded. Subjects selfselecting pessary were compared to those with no intervention during the study period. Followup was performed after a minimum of 3 weeks of treatment. The proportion of subjects demonstrating cure or improvement was compared using a chi-square test. The IRB at the University of Southern California approved this protocol. This project was supported by a grant from the American Institute of Ultrasound in Medicine.

Results: 180 subjects were enrolled. 55 had some degree of hydronephrosis for a prevalence of $30.6 \%$ (24.3\%-37.6\%). 2 were excluded due to surgical intervention before first followup. Of those with hydronephrosis: 37 (69.8\%) chose pessary while 16 (30.2\%) chose expectant management. There was no difference between groups in age, BMI, parity, race, ethnicity, menopausal status or uterine size. Of those who elected pessary, 24 (64.9\%) were using a pessary at the time of their followup. Four subjects were lost to followup ( 3 no treatment, 1 pessary, $p=0.07$ ). The median interval from treatment initiation to repeat ultrasound was 44 (21-325) days. Intention to treat and on treatment results are shown below.

Conclusion: Successful pessary placement improves hydronephrosis in almost $80 \%$ of women with advanced POP. Approximately $35 \%$ of patients may not comply with pessary use and do not demonstrate hydronephrosis improvement.

Clinical Relevance: Pelvic Organ Prolapse

\begin{tabular}{|l|l|l|l|}
\hline Intention to treat analysis & No treatment & Pessary & P \\
\hline Complete resolution (both kidncys) & $4(30.8 \%)$ & $10(27.8 \%)$ & 0.84 \\
\hline Partial resolution & $2(15.4 \%)$ & $13(36.1 \%)$ & 0.17 \\
\hline Any improvement (complete + partial resolution) & $6(46.1 \%)$ & $23(63.8 \%)$ & 0.27 \\
\hline On treatment analysis & & & \\
\hline Complete resolution (both kidneys) & $6(24 \%)$ & $8(33.3 \%)$ & 0.47 \\
\hline Partial resolution & $4(16 \%)$ & $11(45.8 \%)$ & 0.02 \\
\hline Any improvement (complete + partial resolution) & $10(40 \%)$ & $19(79.2 \%)$ & 0.005 \\
\hline
\end{tabular}

\section{Podium \#27}

ELECTRICAL STIMULATION OF SOMATIC AFFERENT NERVES IN THE FOOT INCREASES BLADDER CAPACITY IN HEALTHY HUMAN SUBJECTS

Mang Chen, $\mathbf{M D}^{1}$, Christopher Chermansky, $\mathbf{M D}^{1}$, Bing Shen, DVM ${ }^{1}$, James Roppolo, $\mathrm{PhD}^{2}$, William de Groat, $\mathrm{PhD}^{2}$ and Changfeng Tai, $\mathrm{PhD}^{3}$ ${ }^{1}$ Department of Urology, University of Pittsburgh School of Medicine; ${ }^{2}$ Department of Pharmacology, University of Pittsburgh School of Medicine; ${ }^{3}$ Departments of Urology and Pharmacology, University of Pittsburgh School of Medicine (Presented by: Christopher Chermansky, MD) 
Introduction and Objectives: Treatments for Overactive Bladder $(\mathrm{OAB})$ patients that have failed behavioral and antimuscarinic therapies include intradetrusor injection of onabotulinumtoxinA, sacral neuromodulation, or tibial neuromodulation. Yet, a non-invasive and convenient OAB treatment with no major adverse effects would be very attractive to many patients. Previous cat studies have demonstrated inhibited reflex micturition and increased bladder capacity with transcutaneous electrical stimulation of somatic afferent nerves in the foot using skin surface electrodes. We sought to determine if electrical stimulation of somatic afferent nerves in the foot can delay bladder filling sensations and increase bladder capacity in healthy humans without OAB.

Methods: Eight subjects underwent 90 minute foot stimulation using skin surface electrodes connected to a transcutaneous electrical nerve stimulator. The electrodes were attached to the bottom of the foot to activate the lateral and medial plantar nerves. Subjects completed a 3-day voiding diary during which foot stimulation was applied on the second day. The subjects were provided $500-1000 \mathrm{~mL}$ of water to drink during stimulation. Stimulation parameters included a pulse frequency of $5 \mathrm{~Hz}$, a pulse width of $0.2 \mathrm{~ms}$, and an intensity of 2-6 times the minimal stimulation current necessary to induce a toe twitch. The stimulation intensity was maximally set by the subjects themselves to ensure comfort.

Results: Obtained: The stimulation intensity varied from $2 \mathrm{~T}$ to $3 \mathrm{~T}$ in 7 subjects and $6 \mathrm{~T}$ in the last subject. The average volume per void was $350 \pm 22 \mathrm{~mL}$ during the 24 hour period prior to foot stimulation (See Figure). The voided volume increased to $547 \pm 52 \mathrm{~mL}(\mathrm{p}<0.01)$ for up to 5 hours after stimulation. The average voided volume returned to $363 \pm 21 \mathrm{~mL}$ within 36 hours after stimulation. There were no adverse events.

Conclusion: Foot stimulation did delay bladder filling sensations and significantly increase bladder capacities in 8 healthy humans without OAB. Although the study group was small, our results support moving forward with clinical trials of foot stimulation in OAB patients.

Funded by University of Pittsburgh School of Medicine Department of Urology

Clinical Relevance: Neuromodulation

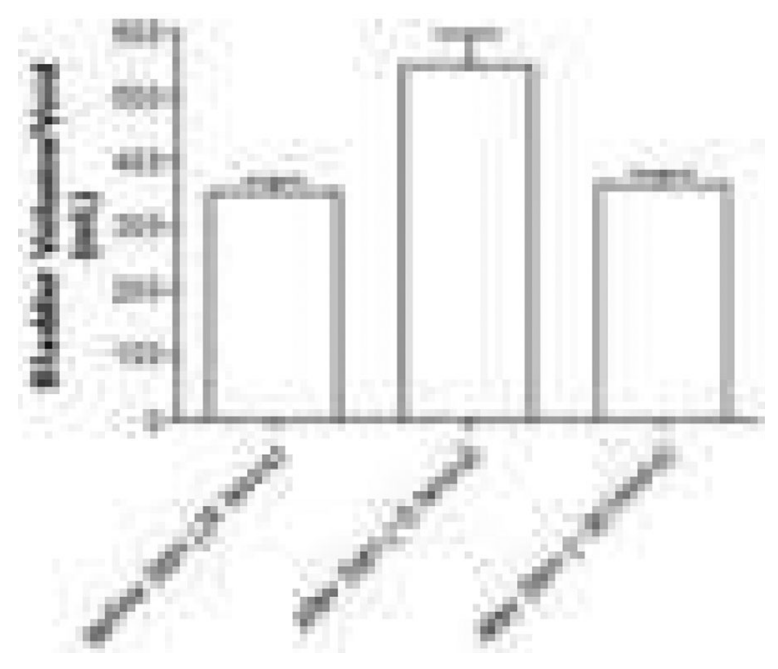

Podium \#28

ANALYSIS OF CROSS-OVER RATES BETWEEN SACRAL NEUROMODULATION AND ONABOTULINUMTOXIN-A INJECTION IN REFRACTORY IDIOPATHIC OVERACTIVE BLADDER
Sara Lenherr, MD, Duncan Morhardt, MD, PhD, Heather Crossley, MS, Cynthia Stroup, PA, Anne Cameron, MD, Ann Oldendorf, MD, John Stoffel, MD, J Quentin Clemens, MD, MS

University of Michigan, Ann Arbor, MI

(Presented by: Sara Lenherr, MD)

Introduction: Many patients with refractory $\mathrm{OAB}$ undergo treatment with sacral neuromodulation (SNM) or intradetrusor OnabotulinumtoxinA (BTXA) injections. While both techniques offer distinct advantages and risks, some patients cross-over from one treatment to the other when therapeutic benefit is not achieved. Our aim was to assess the outcomes of our patients who crossed over from one treatment to the other.

Methods: We retrospectively reviewed all idiopathic overactive bladder (iOAB) patients treated at our institution with SNM and BTXA from February 2004 to September 2013. After sacroneuromodulation lead testing, permanent device was placed if $>50 \%$ improvement in symptoms was observed over the test period. BTXA units were determined by the surgeon on a case-by-case basis. Therapeutic failure was defined as an increase in medication regimen, an additional procedure or patient reported dissatisfaction with the treatment.

Results: Of 29 unique patients, 19 patients started with SNM and 10 started with BTXA. 86\% of the group were female. After crossing over to the other technology, 4 of the 19 SNM to BTXA and 3 of the 10 BTXA to SNM were successful, thus only $24 \%$ $(n=7)$ had successful treatment overall. Mean age at time of first treatment was $58.6 \pm 16.5$ years and mean BMI was $34.3 \pm 9.1 \mathrm{~kg}$. Median time between crossover to the alternate form of neuromodulation was 12.4 months (range 0.9-68 months). Mean duration of follow-up was $38 \pm 26$ months. BTXA dosage was similar between both groups with an overall mean dosage of $200 \pm 82$ units. Fifty-five percent $(n=16)$ of patients underwent multiple BTXA injections (7 patients BTXA to SNM, 9 patients SNM to BTXA).

Conclusion: In our practice, most $\mathrm{OAAB}$ patients that underwent both neuromodulation methods started with SNM and transitioned to BTXA. With only a $24 \%$ success rate overall, patients who have failed one form of neuromodulation had a high probability of failed management with the other treatment. Limited sample size precludes determining specific patient characteristics predicting success with one form of neuromodulation over the other.

Clinical Relevance: Female Urology-including Incontinence

\section{Podium \#29}

\section{INTERSTIM DEVICE INSERTION RATES FOLLOWING PERIPHERAL NERVE EVALUATION VS. STAGE ONE}

Vani Dandolu, MD, MPH, MBA, Sneha Sura, MS

University of Nevada School of Medicine

(Presented by: Vani Dandolu, MD, MPH, MBA)

Objective: to examine the rate of sacral neuro-modulation (SNM) device insertion rates for patients that started with Peripheral Nerve Evaluation (PNE) vs. those that started with Stage One

Methods: The data for this study were obtained from the 20012010 US Thomson Reuters MarketScan Commercial Claims and Encounters database. The database is composed of detailed information about clinical utilization, and expenditures across inpatient, outpatient, emergency room and prescription drug claims of individuals enrolled in employer-sponsored and public health insurance plans. Study cohort included patients 
over 18 years of age who were evaluated for sacral nerve stimulation procedure. Subjects were identified from inpatient admission, inpatient services, outpatient services and facility header files using CPT codes. All statistical analyses were performed using SAS 9.3.

Results: There were 2,374 patients that started directly as stage 1 procedures and 3,775 that started as PNE between January 1, 2001 and December 31, 2010. Among patients that started as Stage One, 1,630 progressed to generator insertion (68.66\%). Of the patients that started with PNE test procedure, only 259 (6.86\%) progressed to generator insertion directly. About 1450 (38.41\%) dropped off after PNE test procedure. Remaining 2066 $(54.73 \%)$ subjects went onto have stage 1 test procedure and 1648 (43.66\%) of them progressed to generator insertion. The rate of eventually getting generator for the subset of patients that started as PNE was $50.52 \%(n=1907)$. There are significant cost differences between these two approaches. Per 2013 Medicare fee schedule the provider fee for stage 1 is $\$ 695$ and for generator implantation is $\$ 247$ resulting in total reimbursement of $\$ 942$ with direct stage 1 approach. Provider fee for PNE is $\$ 1469$ (professional fee $\$ 856$ and $\$ 613$ facility fee). For cases that proceed to stage 2 there is an additional payment of $\$ 858$. For those that preceded to stage 1 and then stage 2 the provider fee will maximize at $\$ 2410.47$.

Conclusion: PNE as initial SNM evaluation procedure is more popular than direct stage 1. However, rate of positive response with PNE seems to be low. Provider fee associated with PNE approach is significantly higher compared to direct stage 1 . Overall health care cost related to SNM can be reduced by direct stage 1 approach. Patients also have a better chance of getting the generator placed if they directly started from stage 1 .

Funding: Institutional funds

Clinical Relevance: Neuromodulation

\section{Podium \#30}

\section{EFFICACY OF PERCUTANEOUS TIBIAL NERVE STIMULATION (PTNS) FOR OVERACTIVE BLADDER IN A COMMUNITY-BASED UROLOGY PRACTICE}

Scott MacDiarmid, MD, Karen Michael, RN

Alliance Urology Specialists

(Presented by: Scott MacDiarmid, MD)

Purpose: The efficacy and safety of percutaneous tibial nerve stimulation (PTNS) with the Urgent ${ }^{\mathbb{R}}$ PC Neuromodulation System for overactive bladder (OAB) has been well established in randomized controlled trials with level 1 evidence. The average age of patients in most OAB studies is generally 58-62 years. In this study we evaluated the efficacy of PTNS in a realworld clinical setting of a large cohort of generally older patients.

Methods: A retrospective analysis of electronic medical records from May 2008 to August 2013 at a single center was completed on 286 OAB patients who underwent 12 PTNS treatments. Following the initial treatments, patients in consultation with their physicians determined if they were "Responders"and should move forward to receive on-going therapy at approximately monthly intervals. Patients completed 7-day voiding diaries at baseline and prior to each of their 12 treatments. Median OAB weekly episodes of daytime frequency, nocturia, and urge incontinence were recorded.

Results: This patient population consisted of $64.3 \%$ females $(184 / 286)$ and $35.7 \%$ males (102/286) with a median age of 78.5 years (range 19-96 yr.). 10.8\% (31/286) of patients had a neurological impairment and all had failed at least two or more antimuscarinic agents. 256 of the 286 (89.5\%) patients completed 12 weeks of treatment. At or before 12 weeks, 59/ 286 (20.6\%) discontinued PTNS therapy for various reasons (19 ineffective treatment, 15 unknown, 12 other health issues, 7 insurance/cost, 3 family issues, 2 transportation/moved, 1 alternative treatment). Of the 286 patients who initially started treatment 218 (76.2\%) desired to continue with on-going PTNS therapy.

Conclusion: The study supports the efficacy of PTNS in a large cohort of generally older patients, and is similar to those in well-designed, level 1 clinical trials. Following the initial 12 weekly treatments, $76.2 \%$ of patients desired to continue with on-going therapy.

Source of Funding: None

Clinical Relevance: Urodynamics

\begin{tabular}{|c|c|c|c|}
\hline \multicolumn{4}{|c|}{ 7-Day Voiding Diary Episodes Per Day; mean \pm SD (n); median [min, max] } \\
\hline Visit & Daytime Frequency & Nocturia* & Urge Incontinence ${ }^{*}$ \\
\hline Baseline & $\begin{array}{l}9.8 \pm 4.2(286) \\
9.0[3.0,32.0]\end{array}$ & $\begin{array}{l}3.5 \pm 2.0(278) \\
3.0[1,0,13.0]\end{array}$ & $\begin{array}{l}3.5 \pm 3.0(248) \\
3.0[1.0,16.0]\end{array}$ \\
\hline $\begin{array}{l}\text { Completing diary } \\
\& 12 \text { PTNS Tx }\end{array}$ & $\begin{array}{l}6.8 \pm 2.6(256) \\
6.0[2.0,29.0]\end{array}$ & $\begin{array}{l}2.0 \pm 1.7(249) \\
2.0[0.0,15.0]\end{array}$ & $\begin{array}{l}1.6 \pm 1.9(224) \\
1.0[0.0,15.0]\end{array}$ \\
\hline
\end{tabular}

\section{Podium \#31}

INFECTION RATE AT 12 MONTHS IN A LARGE PROSPECTIVE TRIAL OF SACRAL NEUROMODULATION FOR OVERACTIVE BLADDER PATIENTS

Jason Bennett, $M D$, Jeffrey Mangel, $M^{1}$, Craig Comiter, $M D^{2}$, Samuel Zylstra, $\mathrm{MD}, \mathrm{MPH}^{3}$, Erin Bird, $\mathrm{MD}^{4}$,

Tomas L. Griebling, $\mathrm{MD}, \mathrm{MPH}^{5}$, Daniel Culkin, $\mathrm{MD}^{6}$, Suzette E. Sutherland, $\mathbf{M D}^{7}$, Karen Noblett, $\mathbf{M D}^{8}$, Kellie Chase Berg, MS $^{9}$, Fangyu Kan, MS $^{9}$ and Steven Siegel, $\mathrm{MD}^{10}$

${ }^{1}$ MetroHealth Medical Center, Cleveland, $\mathrm{OH} ;{ }^{2}$ Stanford University, Stanford, CA; ${ }^{3}$ Milford Regional Medical Center, Whitinsville, MA; ${ }^{4}$ Scott and White Healthcare, Temple, TX; ${ }^{5}$ University of Kansas, Kansas City, KS; ${ }^{6}$ University of Oklahoma HSC, Oklahoma City, OK; ${ }^{7}$ University of Washington, Seattle, WA; ${ }^{8}$ University of California, Irvine, CA; ${ }^{9}$ Medtronic, Inc., Minneapolis, $M N ;{ }^{1}$ Centers for Continence Care and Female Urology, Metro Urology, Woodbury, MN

(Presented by: Jason Bennett, MD)

Introduction: This prospective, multicenter post-approval study evaluated the 12 month infection rate in subjects receiving sacral neuromodulation (SNM) therapy with the InterStim ${ }^{\circledR}$ System (models 3023 and 3058). This analysis represents a cohort of subjects implanted using a minimally invasive technique with a tined lead (models 3889 and 3093). The InSite $O A B$ trial enrolled subjects with bothersome symptoms of overactive bladder (OAB) including urinary urge incontinence (UI) or urgency-frequency (UF), who had failed at least 1 anticholinergic medication but had at least 1 medication not yet tried.

Methods: Enrolled subjects discontinued OAB medications 4 days before and during completion of baseline voiding diaries. Subjects with successful test stimulation received an SNM implant. Adverse events are reported by investigators at scheduled and unscheduled visits. Infection rate at 12 months associated with any device component (neurostimulator, lead and/or extension) in subjects with a full system implant is reported. All adverse events included were reviewed by a Clinical Events Committee. The protocol did not require specific 
measures for infection control but subjects could have prophylactic oral antibiotics if needed.

Results: Of the 340 enrolled subjects that completed test stimulation, 272 were implanted with SNM. Mean age was 57 years and $91 \%$ were female. Infection rate at 12 months post full system implant was 3.7\%. From full system implant through 12 months, 10 subjects experienced 13 adverse events for infection, including 12 implant site infection and 1 cellulitis. None met the definition for serious. Ten of the 13 events occurred prior to 6-months. Seven subjects had 1 event each and three subjects had 2 events each. Ten events were located at the neurostimulator pocket, 2 at the lead tract and 1 at the neurostimulator pocket, lead tract and lead introducer site. Eight events were resolved by device surgical intervention (5 permanent explants and 3 device replacements) and 5 events were resolved by medical/non-surgical therapy or other surgical treatment. The overall rate of infection requiring surgical intervention was $2.9 \%(8 / 272)$ and the rate of permanent explant due to infection was $1.8 \%(5 / 272)$.

Conclusion: These data from the multi-center InSite OAB trial demonstrate a low adverse event rate for infection at 12months. This currently used minimally invasive surgical approach has improved infection rates compared to the original procedure.

Clinical Relevance: Neuromodulation

\section{Podium \#32}

\section{ADVANCE SLING USAGE IN MALE NEUROGENIC PATIENTS WITH INTRINSIC SPHINCTER DEFICIENCY: A DURABLE TREATMENT}

Karl Coutinho, MD, Jonathan Vapnek, MD, Neil Grafstein, MD Mount Sinai Hospital, New York, NY

(Presented by: Karl Coutinho, MD)

Introduction and Objectives: Stress urinary incontinence (SUI) can be a debilitating problem in neurogenic patients with sphincter dysfunction, causing discomfort, embarrassment, and social anxiety. Urethral suspension using a male sling may be beneficial to these patients' continence outcomes. We report long term follow up from our initial experience using male slings to treat SUI in adult neurogenic patients with intrinsic sphincter deficiency.

Materials and Methods: Since 2010, we have followed five adult neurogenic patients ages 18-49 who were offered AdVance male slings to treat SUI occurring between selfcatheterizations. All five suffered from neurogenic sphincteric deficiency and SUI. They used an average of 3.6 pads per day (+/-1 pad), and leaked during strenuous activity and transfers. Two underwent bladder augments in addition to a male sling. Median follow-up was 16 months (range 3 to 25). Data gathering was through review of medical records and phone interviews. ICIO and subjective parameters were used evaluate outcomes.

Results: All patients reported resolution of their SUI after receiving the AdVance male sling. Average pre and postoperative ICIO scores were 17 and 1.25 respectively. Average reduction in ICIO score was 15.7 (SD of 3.1, Median of 15.5), unchanged from our previous follow up one year prior. Postoperative pad use has been reduced to zero in all patients. All patients reported the operation as a "success" and reported that they would recommend the procedure to others with their condition.

Conclusion: The AdVance male sling is an effective way to treat neurogenic patients with SUI from sphincter deficiency. In contrast to post prostatectomy patients, its success in neurogenic patients with no prior prostate or urethral surgery suggests a compressive component to the operation, sufficient enough to provide measurable results. Our study represents the longest published follow-up for these patients, indicating a durability of this effect. These results may be comparable with the gold standard, Artificial Urinary Sphincters, which carry a higher rate of revision, especially through the lifetime of these typically younger patients. In our small sample, the results, both subjective and objective, show a benefit and durability of male sling usage in these neurogenic patients.

clinical Relevance: Male Incontinence

\section{Podium \#33 \\ PATIENTS WITH NEUROGENIC DETRUSOR OVERACTIVITY TREATED WITH ONABOTULINUMTOXINA ACHIEVE THEIR TREATMENT GOALS}

Eric Rovner, $\mathrm{MD}^{1}$, Michael Chancellor, $\mathrm{MD}^{2}$, Catherine Corbell, $\mathrm{PhD}^{3}$, Ouanhong $\mathrm{Ni}, \mathrm{MS}^{3}$, Denise Globe, $\mathrm{PhD}^{3}$ and Emmanual Chartier-Kastler, $\mathrm{MD}^{4}$ ${ }^{1}$ Medical University of South Carolina, Charleston, SC; ${ }^{2}$ Oakland University, William Beaumont School of Medicine, Royal Oak, MI; ${ }^{3}$ Allergan, Inc., Irvine, CA; ${ }^{4}$ Université Paris-VI, PitiéSalpétrière Academic Hospital, Paris, France

(Presented by: Eric Rovner, MD)

Introduction and Objectives: Patient-reported goals for treatment of urinary incontinence (UI) due to neurogenic detrusor overactivity (NDO) are not well documented. The objectives of our analysis were to identify the treatment goals of patients (pts) with UI due to NDO and determine whether pts achieved their goals following onabotulinumtoxinA (onabotA) treatment.

Methods: Data from two phase 3, placebo-controlled, doubleblind, randomized studies were pooled for analysis of treatment goals (at study baseline), and to determine the proportions of pts who achieved their goals 6 weeks after treatment with onabotA 200U. Eligible pts had NDO ( $\geq 14$ UI episodes/wk) due to multiple sclerosis (MS) or spinal cord injury (SCI) and were not adequately managed by anticholinergics. Our study population consisted of all pts who received onabotA $200 \mathrm{U}$ at study entry. At study entry, pts were asked to list their top 1 or 2 primary goals for treatment of their overactive bladder. Qualitative responses were then grouped into symptom-and quality of life-related categories. Six weeks after onabotA treatment, pts were asked to rate how effectively onabotA helped them achieve their stated goals (pt response of significant progress toward or complete achievement of goals). We analyzed the frequency distribution of goals at study entry and the percent of pts who achieved their goals stratified by neurological disease.

Results: Of the study population ( $\mathrm{N}=221), 128$ (57.9\%) were, MS pts and 93 (42.1\%) were SCI pts. A total of 219 pts $(99.1 \%$ of study population) enumerated their treatment goals at baseline. Most common treatment goals were "to be dry" $37.0 \%$ of pts), "to reduce UI therapies"(19.6\%) and "to improve bladder control"(15.1\%). Overall, $60.7 \%$ of pts achieved at least 1 of their treatment goals 6 weeks after onabotA 200U treatment; 50.6\% achieved their goal of being dry, $44.2 \%$ achieved their goal of reducing UI therapies and $66.7 \%$ achieved their goal of improved bladder control. There were no major differences in the overall percent of pts who achieved at least one of their treatment goals by neurological disease (62.7\% for, MS vs $58.1 \%$ for SCI). 
Conclusion: A majority of pts with UI due to NDO achieved their goals following onabotA $200 U$ treatment. Identification of individual treatment goals may allow clinicians to more effectively evaluate therapy, inform patient treatment decision making, and enhance patient satisfaction and treatment benefit. Funded by Allergan, Inc.

\section{Podium \#34}

SIMPLE CYSTECTOMIES FOR BENIGN DISEASE: RESULTS: OF A 10-YEAR RETROSPECTIVE STUDY.

David Osborn, MD, Stephen Mock, MD, Nicholas Smith, Catherine Harris, MD, Osa Okunbur, MD, Xuechao Zhang, Michael Guan, Melissa Kaufman, MD, PhD,

W. Stuart Reynolds, MD, Roger Dmochowski, MD

Nashville, TN

(Presented by: David Osborn, MD)

Introduction: The primary goal of a cystectomy for benign disease is to improve quality of life of patients with diseases such as spinal cord injury, spina bifida, multiple sclerosis and radiation damage to the bladder while minimizing patient complications. The purpose of this study was to analyze what factors contribute to a worse outcome after surgery measured by frequency of severe complications.

Materials and Methods: A retrospective review was performed of consecutive patients who underwent a cystectomy for benign disease. Parameters investigated included demographic information, comorbid medical conditions, Charlson comorbidity index, type of surgery performed and operative and postoperative course. The primary outcome measure was frequency and severity of complications (measured using Clavien-Dindo classification system). The association between postoperative complications and the independent variables was assessed with multivariate logistic regression.

Results: A total of 139 patients underwent cystectomy for benign diseases during the study period. Mean age was 52 years and $59 \%$ of the patients were women. The most common indications for surgery were spinal cord injury (32\%) and radiation damage to the bladder (21\%). Seventy-four (53\%) patients underwent supratrigonal cystectomy. The average length of surgery was 344 minutes and estimated blood loss was $476 \mathrm{ml}$. The most common complications were prolonged ileus and pyelonephritis. Seventy-nine (57\%) patients had a complication classified as 2 or greater on the Clavien-Dindo scale and $36(26 \%)$ had a complication greater than 2. After adjustment, length of surgery in minutes (OR 1.01, 95\% CI 1.001.01, p 0.004) and Charlson comorbidity index (OR 1.26, 95\% CI $1.03-1.53, \mathrm{p} 0.024)$ were associated with an increased incidence of a Clavien-Dindo 2 or greater complication.

Conclusion: The complications of simple cystectomy for benign disease are comparable to those of radical cystectomy done for the indication of malignancy published in the literature. Similar to other studies examining the outcomes of a surgical procedure, length of surgery is an important variable that can affect outcome. In addition, not surprisingly Charlson comorbidity index is also correlated with a worse outcome. This study supports the statement that surgeons can use information about a patient's preoperative health to guide counseling about the risks of postoperative adverse events.

Clinical Relevance: Neurogenic Bladder

\section{Podium \#35}

THE VALIDITY AND RELIABILITY OF THE NEUROGENIC BLADDER SYMPTOM SCORE
Blayne Welk, MD, $\mathrm{MSc}^{1}$, Sarah Morrow, MD, $\mathrm{MSc}^{2}$, Wendy Madarasz RD MPE${ }^{3}$, Richard Baverstock, $\mathrm{MD}^{4}$, Jennifer Mcnab, $\mathrm{PhD}^{5}$ and Keith Sequeira, $\mathbf{M D}^{6}$

${ }^{1}$ Department of Surgery, Western University, London ON; ${ }^{2}$ Department of Clinical Neurosciences, Western University; ${ }^{3}$ St Joseph's Health Care, London Ontario, Canada; ${ }^{4}$ vesia [Alberta Bladder Centre], Division of Urology, Department of Surgery, University of Calgary; ${ }^{5}$ Department of Epidemiology and Biostatistics, Western University; ${ }^{6}$ Department of Physical Medicine and Rehabilitation, Western University

(Presented by: Blayne Welk, MD, MSc)

Objectives: The neurogenic bladder symptom score (NBSS) is a tool to measure urinary symptoms and consequences among patients with acquired or congenital neurogenic bladder. This objective of this study was to describe the validity and reliability of the NBSS.

Methods: Exploratory factor analysis was used to assess item variability and subscale structure. Reliability was assessed with Cronbach's alpha, and correlation with retest data. Validity was assessed with a priori hypotheses specifying relationships with the American urologic association symptom score (AUA-SS), International Consultation on Incontinence Questionnaires-Urinary Incontinence (ICIO-UI), and urinary specific quality of life (SF-Oualiveen) and a self-assessed global bladder problem score. Known groups analysis was used to further assess construct validity.

Results: A cohort of 230 patients with spinal cord injury, (35\%), multiple sclerosis (59\%), and congenital neurogenic bladder (6\%) were included in this study. Factor analysis suggested 3 domains within the NBSS (incontinence, storage and voiding symptoms, and consequences). Overall internal consistency was high, with a Cronbach's alpha of 0.89. Test-rest reliability was also excellent, with an intraclass correlation coefficient of 0.91 . Validity was demonstrated with the confirmation of hypothesized correlations with the AUA-SS, ICIQ-UI, and SFOualiveen, and significant differences in NBSS scores among known groups (those with a history of seeing a urologist had a significantly higher mean score (22.1 vs 17.1, $p<0.001$ ), as did those who had a higher global bladder problem score (22.1 vs 12.6, $\mathrm{p}<0.001)$ ).

Conclusion: The NBSS, developed specifically to assess the symptoms and consequences associated with neurogenic bladder dysfunction, has appropriate psychometric properties for use in clinical study.

Acknowledgement: This investigator-driven research was supported by the Canadian Urological Association Astellas Research Grant Program.

Clinical Relevance: Neurogenic Bladder

\section{Podium \#36}

LONG-TERM RESULTS: OF AUGMENTATION ENTEROCYSTOPLASTY IN PATIENTS WITH CHRONIC PROGRESSIVE MULTIPLE SCLEROSIS

Daniel Nassau, Jerry Blaivas, MD

New York, NY

(Presented by: Daniel Nassau, MD)

Introduction: There is little data on the long-term management of refractory neurogenic voiding dysfunction (NVD) in patients with chronic progressive Multiple Sclerosis (MS). We reviewed long-term outcomes in, MS patients who underwent augmentation enterocystoplasty (EC). 
Methods: This is a retrospective observational study of consecutive patients (1984-2008) with, MS and NVD who underwent EC with or without a continent/incontinent abdominal stoma. Preoperative evaluation included routine labs \& vitamin B12 levels, videourodynamic study(VUDS), cystoscopy, and upper-tract imaging. Postoperative evaluation comprised bi-annual office visits (alternating VUDS and renal/ bladder ultrasound). Cystoscopy was performed only for specific indications. Long-term outcomes and complications were assessed by validated questionnaires and chart review including pre and post op VUDS.

Results: 17 subjects (13 women, 4 men) with chronic progressive, MS underwent EC. Thirteen were wheelchair bound (11 quadriplegic). One was excluded when it was found that she did not have, MS and two did not attend post op visits and were followed by telephone. Mean age was 52 years (3470) with a median follow-up of 5 years (1-17). Indications included detrusor-external sphincter dyssynergia (10), detrusor overactivity (12) \& low bladder compliance (5), often in combination. A colonic segment was used 3 patients, an ileocecal in 6 and an ileal in 7. Five patients had a continent stoma, 5 had an ileovesicostomy \& 2 underwent pubovaginal sling. One patient committed suicide 3 months post op, and 7 died during follow-up. 12 patients (86\%) had a successful outcome on validated questionnaires. All, except those with ileovesicostomy, required intermittent self-catheterization. Average bladder capacity increased from 190 to $622 \mathrm{~mL}$ and max detrusor pressure decreased from 61 to $14 \mathrm{~cm} \mathrm{H2O}$. All had normal creatinine and B12 levels on follow-up. One patient needed operative revision for stomal stenosis. Two patients experienced small bowel obstruction 3 and 7 years after surgery one of whom required lysis of adhesions. One patient developed recurrent febrile UTIs. No patient developed hydronephrosis, troublesome diarrhea, B12 deficiency, de novo urolithiasis or cancer in the augmented bowel but one had nephrogenic adenoma.

Conclusion: Augmentation EC is a safe, effective, and we believe underutilized procedure for refractory NVD in, MS patients.

\section{Podium \#37}

URINARY PHYTOESTROGEN LEVELS ARE ASSOCIATED WITH LOWER URINARY TRACT SYMPTOMS IN POSTMENOPAUSAL WOMEN

Evgeniy Kreydin, $\mathrm{MD}^{1}$, Yahir Santiago-Lastra, $\mathrm{MD}^{1}$, Dayron Rodriguez, $\mathrm{MD}, \mathrm{MPH}^{1}$, Alejandro Sanchez, $\mathrm{MD}^{1}$, Hillary Keenan, PhD $^{2}$ and Dicken Ko, MD, FRCSC ${ }^{1}$

${ }^{1}$ Massachusetts General Hospital, Boston, MA; ${ }^{2}$ Joslin Diabetes Center, Boston, $M A$

(Presented by: Evgeniy Kreydin, MD)

Introduction: Phytoestrogens are a class of plant-derived biological compounds that have selective estrogen receptor modulator properties. Links have been established between urinary phytoestrogen levels and a number of sex hormonedependent pathologic states, such as menopause-associated vaginal atrophy and osteoporosis. We examined whether urinary levels of isoflavone-type (daidzein, o-desmethylangolensin, equol, and genistein) and lignan-type phytoestrogens (enterodiol and enterolactone) were associated with urinary symptoms in postmenopausal women.

Methods: National Health and Nutrition Examination Survey (NHANES) data collected from 2000 to 2010 was used to identify a cohort of 1870 postmenopausal women, who responded to a urinary incontinence questionnaire and 734 postmenopausal women, who responded to a nocturia questionnaire. Phytoestrogen levels were normalized to urinary creatinine concentration and log transformed. Association between phytoestrogen levels, urge incontinence, stress incontinence, other incontinence, and nocturia was assessed using an unweighted multivariate logistic regression adjusting for age, race, smoking status, body mass index, diabetes and parity. Results: Increasing concentration of lignan phytoestrogens enterolactone $(p<0.05)$ and enterodiol $(p<0.10)$ was associated with decreased likelihood of urge incontinence and other incontinence. Increasing concentration of these phytoestrogens was also associated with decreased likelihood of nocturia $(p<0.05)$. Increasing concentration of isoflavone genistein was associated with increased likelihood of urge incontinence $(p<0.05)$; otherwise isoflavone levels were not associated with any urinary symptoms $(p>0.10)$. Odds ratios for each association are given in Table 1.

Conclusion: This is the first study to demonstrate an association between urinary phytoestrogen levels and urinary symptoms in a large cohort of postmenopausal women. We demonstrate that members of the lignan phytoestrogen family may have a protective effect on urinary symptoms in this population. Prospective clinical and laboratory studies are warranted to investigate the mechanism of this relationship. Clinical Relevance: Female Urology-including Incontinence

\begin{tabular}{|c|c|c|c|c|c|}
\hline $\begin{array}{l}\text { PHTTOESTROGEN } \\
\text { TME }\end{array}$ & PHTTOESTROGEN & 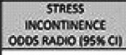 & 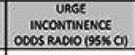 & 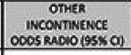 & 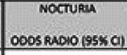 \\
\hline BOFLANONE & DNOZEIN & 0.8586 (a.4.46.1.81) & $1.71(0.802 \cdot 3.48)$ & 1.39 (a.521-3.69) & $1.18(0.323 \cdot 1.16)$ \\
\hline BOFLUNONE & O.DESMETRTUNGOLENSIN & $1.10(0.645 .1 .59)$ & $1.35\{(0.782 .2 .31)$ & $1.06(0.494-2.22)$ & $1.35(0.430-4.35)$ \\
\hline SOFLAVONE & Equar & $0.8966(0.340-2.33)$ & 0.751 (0.188-2.83) & $1.10(0.186-6.01)$ & $1.14(0,441-2.95)$ \\
\hline BORLWVONE & GENISTEN & $\alpha=876(0.473-1.62)$ & $2.11(1.13 \cdot 3.349)=$ & $1.47(0.622-3.45)$ & $1.54(0.425-5.41)$ \\
\hline LGEAN & ENTEROOIOL & $1.06(0.459-2.44)$ & $0.471(0.202 .109)^{*}$ & $0.009(0.136 \cdot 1.25)^{*}$ & $0.200(0.440 .942) \cdots$ \\
\hline LENAN & IMTEROUACTONE & $0.629(0.288-1.37)$ & $0.387(0.177 .0 .847)^{*}$ & $0.262(0.095-0.740)^{*}=$ & $0.116(0.031-0.444) * *$ \\
\hline
\end{tabular}

\section{Podium \#39}

\section{SALIVARY CORTISOL RESPONSE TO STRESS IN WOMEN WITH OVERACTIVE BLADDER AND CONTROLS}

Ariana Smith, $\mathrm{MD}^{1}$, Liisa Hantsoo, $\mathrm{PhD}^{2}$ and C. Neill Epperson, $M^{2}$

${ }^{1}$ Division of Urology, University of Pennsylvania, Philadelphia, PA; ${ }^{2}$ Department of Psychiatry, Penn Center for Women's Behavioral Wellness, University of Pennsylvania, Philadelphia, PA

(Presented by: Ariana Smith, MD)

Introduction: Physiologic measures of stress response modulated by the hypothylamic pituitary axis may contribute to lower urinary tract symptoms in women with overactive bladder (OAB)

Methods: Postmenopausal women age 45-75 diagnosed with $\mathrm{OAB}$ were recruited from a tertiary care Urology practice and age matched healthy controls were recruited from a research database. Participants were excluded if they were undergoing treatment for stress reduction, using anxiolytic or hormonal medications, reported pain, urinary infection, bladder cancer, or psychiatric disease. Salivary cortisol levels were obtained at baseline and at the time of induced psychosocial stress. The Trier Social Stress Test (TSST) was used as a motivated performance task for the induction of psychological stress. A two-day cortisol awakening response (CAR) and baseline 
salivary cortisol samples were obtained in all participants prior to TSST and at defined intervals post-TSST. Paired T-Tests were used to compare baseline cortisol (obtained 15 minutes before TSST) to stress response cortisol (obtained 50 minutes after TSST) in OAB patients and controls.

Results: Eight patients with $\mathrm{OAB}$ and 5 controls completed the performance task and provided all requested salivary samples. No difference in CAR was detected between OAB women and controls. A trend toward greater physiologic responsiveness in women with $\mathrm{OAB}$ compared to controls was noted (Figure 1, $\mathrm{p}=0.066$ ).

Conclusion: Women with $\mathrm{OAB}$ have similar baseline physiologic measures of stress response to controls. Women with $O A B$ may have greater physiologic responsiveness to stress as measured by salivary cortisol following TSST. Evaluation of markers of stress response may provide targets for potential diagnostic and therapeutic interventions.

Funding: This work was funded by NIH/NIDDK O'Brien Urology Center Grant P50 DK052620-15

Clinical Relevance: Female Urology-including Incontinence

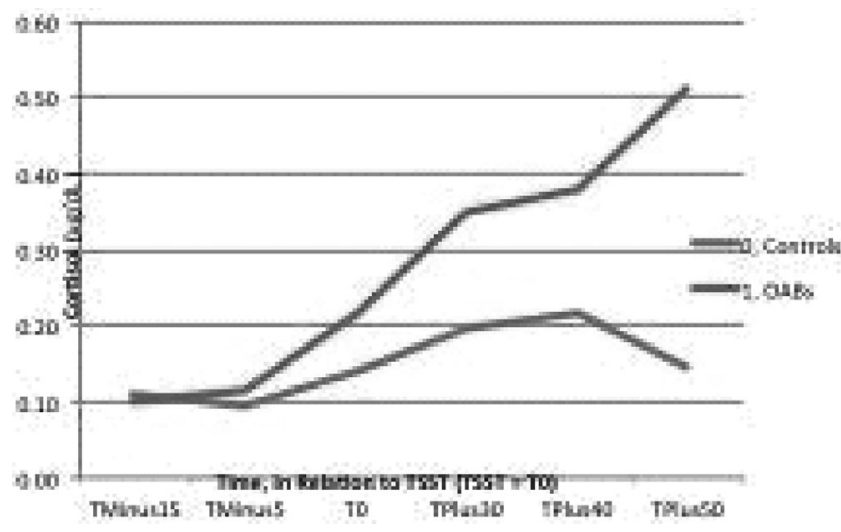

Podium \#40

\section{PATIENT DEFINED GOALS OF CARE IN TREATMENT OF MEDICATION REFRACTORY OVERACTIVE BLADDER}

Mya Levy, MD, Antonette Ayaji BA, Ja-Hong Kim, MD, Z. Chad Baxter, MD, Larissa Rodriguez, MD, Shlomo Raz, MD, Eliav Dahan, MD, Christopher Sagal, MD

UCLA, Los Angeles, CA

(Presented by: Mya Levy, MD)

Introduction: Overactive bladder syndrome $(\mathrm{OAB})$ is a debilitating condition affecting approximately $16.5 \%$ of the United States population. When medication is ineffective, new technologies offer minimally invasive options such as tibilal nerve stimulation, intravesical botulinum toxin injections, or sacral nerve stimulation. In the context of three choices, each with distinctive administration methods, cost, and side effects, but similar efficacy, a patient and clinician are faced with a complex treatment decision, as there is no objectively "right" choice.

Objections: Presently, scientific investigators and clinicians largely define clinical outcomes. We hypothesize that patients have unique, previously ill defined but crucial goals of care (GOC) related to treatment decision-making. Elucidation of these has potential for greater congruence between patients' personal values and treatment choice ultimately leading to improved patient satisfaction.

Methods: We utilized the "Voice of the Customer" methodology taken from marketing science, which provides a structured technique to elucidate patient treatment preferences/GOC and minimize developer/clinician assumptions. We recruited patients with medication refractory $O A B$ from our institution's outpatient urology clinics.

Results: Prospectively, we conducted 15 open-ended patient interviews. 773 unique thoughts were identified from the transcribed interviews. 296 were relevant to OAB treatment. 4 independent reviewers identified 14 GOC themes from these 296 thoughts: Pain with procedure, other people's advice, foreign object inside of your body, invasiveness of treatment, Incontinence/not being dry, foley catheter issues, can't lay down, permanence of treatment, cost of treatment, convenience of treatment, doctor/hospital reputation, side effects of treatment, success rate of treatment, and lifestyle affected by treatment. 51 characteristic quotes were selected that represented the range of levels describing each GOC. 15 additional patients conducted a resorting exercise in order to verify themes and GOC ranges, reducing investigator bias. No additional themes were elucidated.

Conclusion: We were able to comprise a concise set of patient elicited GOC for treatment decision making in OAB. We plan to utilize the above attributes to inform a decision making aid that will effectively characterize an individual's preferences and help guide clinical shared decision making encounters.

Funding source: None

\section{Podium \#41 \\ TRANSURETHRAL RADIOFREOUENCY COLLAGEN DENATURATION FOR THE TREATMENT OF FEMALE STRESS URINARY INCONTINENCE: A SYSTEMATIC REVIEW}

Diana Kang, MD, Julia Han, Philipp Dahm, MD, Louis Moy, MD, Molly Neuberger

Gainesville, FL

(Presented by: Diana Kang, MD)

Introduction and Objectives: To conduct a systematic review that critically evaluates the methodological quality of studies of transurethral radiofrequency collagen denaturation in the treatment of female SUI.

Methods: We conducted a systematic search in August 2011 and updated in September 2012 for randomized and quasirandomized controlled trials of transurethral collagen denaturation in the CENTRAL, PubMed and EMBASE databases. Unpublished studies were sought by hand-searching abstracts of major gynecology and urology meetings from 2003 to 2011, contacting experts in the field, and the device manufacturer. Articles were reviewed for methodological quality by two independent reviewers with arbitration by a third reviewer. The predefined, primary outcome was patient-reported rates of SUI at 12 months. We assessed the quality of evidence using GRADE methodology and performed statistical analysis using RevMan software.

Results: A comprehensive literature search identified 223 original research studies. Twenty four articles were assessed in full-text for eligibility. Eight of the studies (33\%) were observational studies of single-armed cohorts and two studies (8.3\%) were secondary analyses of previously published data. Thirteen review articles (54\%) were excluded as they did not contain original unpublished data. One pre-clinical animal study was excluded. Only one sham-controlled randomized controlled trial of 161 women was included in the analysis. No patient self-reported rates of SUI were found. Treatment with transurethral radiofrequency collagen denaturation was not associated with improved Incontinence Quality of Life (I-OOL) 
scores at 12 months (RR: $1.11 ; 95 \%$ CI $0.77-1.62 ; p=0.73$ ). No serious adverse events occurred in either treatment arm. The quality of evidence was down-graded for study limitations, imprecision and indirectness, and rated as very low overall for all outcomes.

Conclusion: The current best evidence fails to support a beneficial impact of transurethral radiofrequency collagen denaturation on patient-important outcomes thereby questioning its role in urologists' armamentarium to treat SUI. Clinical Relevance: Female Urology-including Incontinence

\section{Podium \#42}

\section{AN EVALUATION OF ADOPTION AND ADHERENCE TO PROPOSED MESH COMPLICATION DEFINITIONS AT PROFESSIONAL SOCIETY MEETINGS}

Chris Tenggardjaja, MD, Adrienne Quirouet, MD, Howard Goldman, MD

The Cleveland Clinic, Cleveland, Ohio

(Presented by: Adrienne Quirouet, MD)

Introduction: In 2011, the International Urogynecological Association (IUGA) and International Continence Society (ICS) proposed newly defined terms, in addition to a classification system, to describe complications of prostheses and grafts in female pelvic floor surgery. Our objective is to evaluate adoption and adherence of these definitions.

Methods: Two reviewers (CT, AO) independently read through all published abstracts presented at SUFU and ICS from 2010 through 2013. Abstracts were included if they dealt with incontinence or prolapse surgery involving prostheses or grafts. Male and animal/bench model testing abstracts were excluded. Discordant data was evaluated separately by a third reviewer (HG). Abstracts were then re-evaluated for the ICS/ IUGA proposed terminology, specifically: erosion, contraction, prominence, separation, exposure, extrusion, compromise, perforation, dehiscence, sinus tract formation. Adherence was the use of proper ICS/IUGA terminology. Non-adherence was the use of "erosion". Hybrid adherence was the use of both. Statistics were performed using Fisher's exact two tailed test.

Results: 1899 abstracts were reviewed. 375 pertained to prostheses and grafts. 137 met inclusion criteria and used terminology related to the ICS/IUGA publication. Of these, 50 (36.5\%) were adherent, 50(36.5\%) were non-adherent, 29 $(21.2 \%)$ were hybrid. Given the availability of the guideline since 2011, we then evaluated 2012 as the cutoff for adoption for the new terminology. A decreased proportion of abstracts contained terminology dealing with complications after publication of the guidelines (40\% vs $49.7 \%, p=0.105$ ). Although a higher proportion of abstracts (24/56,42.9\%) demonstrated adherence to proper terminology after the publication of the guidelines compared to prior 26/81 (32.1\%), this was not statistically significant $(p=0.211)$. The proportion of hybrid abstracts also decreased after Introduction: of the guidelines ( $25.9 \%$ vs $14.3 \%, p=0.136$ ). Programs from SUFU and ICS were then evaluated independently. Both society meetings demonstrated a not statistically significant decrease in the proportion of non-adherent abstracts after 2012.

Conclusion: The minority of abstracts dealing with female pelvic medicine prostheses and grafts adhere to the proposed terminology published by the ICS/IUGA. Although adherence seems to be improving, the value of proposed definitions and standards are questioned if they are not widely adopted.

Clinical Relevance: Female Urology-including Incontinence

\section{Podium \#43}

CONTEMPORARY OUTCOMES IN PATIENTS WITH AND WITHOUT MESH EROSION/EXTRUSION UNDERGOING AUTOLOGOUS FASCIA PUBOVAGINAL SLING AFTER TRANSVAGINAL MESH EXCISION

Samuel Nickles, MD, Taylor Vaughan, MD, Eric Rovner, MD Medical University of South Carolina Charleston, SC (Presented by: Samuel Nickles, MD)

Introduction and Objectives: Patients experiencing persistent incontinence or complications after transvaginal (TV) mesh placement represent a complex population to treat. Autologous fascia pubovaginal sling (AF-PVS) has long been utilized as a "rescue" surgery for those patients with failed prior surgery however the data using this procedure with respect to mesh failures is sparse. This study compared outcomes in patients undergoing autologous fascia pubovaginal sling (AF-PVS) who had mesh erosion into the GU tract and/or vaginal exposure, versus those without erosion or extrusion.

Methods: This was a retrospective analysis of patients who had AF-PVS following TV mesh from January 2007 through March 2013. Overlapping indications for AF-PVS included obstruction $(n=14)$, persistent/worsened incontinence $(n=37)$, mesh extrusion $(n=10)$, or mesh erosion $(n=12)$. Pre-operatively, patients underwent video urodynamics (VUDS), cystoscopy, pad quantity and 24 hour pad weights. Post-operative quality of life (OOL) was compared to pre-operative QOL using a 7point, subjective Likert scale.

Results: 39 patients met inclusion criteria. Of these, 20 patients had a mesh erosion and/or extrusion and 19 did not. Median follow-up was 36 months (range 1-66). Patients with mesh exposure had an increased number of prior mesh surgeries for stress urinary incontinence (SUI) or pelvic organ prolapse (POP) (1.45 vs $1.10, p=0.05$ ) and a higher rate of pre-operative dyspareunia ( $90 \%$ vs $57 \%, p=0.02$ ). There was no significant difference in pre-operative rates of SUI or urge urinary incontinence (UUI), pad number, pad weight, or presence of POP. There was no significant difference in pre-operative UDS findings, except for an increased rate of detrusor overactivity in the mesh erosion/extrusion cohort ( $31.6 \%$ vs $5.3 \%, p=0.03$ ). During the placement of AF-PVS, $20 \%$ of the erosion/extrusion cohort underwent a Martius flap, while no patients in the comparative cohort required a similar procedure $(p=0.04)$.

Conclusion: Post-operatively, both cohorts reported similarly decreased relative pad usage $(-88 \%$ vs $-82 \%, p=0.48)$ and improved OOL. The rate of subsequent SUI and UUI did not differ between the cohorts after AF-PVS (10\% vs $10.5 \%$, $p=0.95$ ). Additionally, there was no statistically significant difference in the presence of POP, dyspareunia, or additional urologic procedures after AF-PVS.

Clinical Relevance: Reconstruction

\section{Podium \#44}

THE FDA AND TRANSVAGINAL MESH: IMPACT ON FELLOWSHIP TRAINING IN FEMALE PELVIC MEDICINE AND RECONSTRUCTIVE SURGERY

Kristie Greene, $\mathrm{MD}^{1}$, Lauren Scott, $\mathrm{MD}^{2}$, Renee Bassaly, $\mathrm{DO}^{3}$, Judette Louis, $\mathrm{MD}^{4}$, Stuart Hart, $\mathrm{MD}^{2}$ and Raul Ordorica, $\mathrm{MD}^{5}$ ${ }^{1}$ Division of Female Pelvic Medicine and Reconstructive Surgery, University of South Florida; ${ }^{2}$ Division of Female Pelvic Medicine and Reconstructive Surgery, University of South Florida, Tampa, Florida; ${ }^{3}$ Division of Female Pelvic Medicine and Reconstructive Surgery, Univeristy of South Florida, Tampa, Florida; ${ }^{4}$ Division of 
Maternal Fetal Medicine, University of South Florida, Tampa, Florida; ${ }^{5}$ Department of Urology, University of South Florida, Tampa, Florida

(Presented by: Kristie Greene, MD)

Introduction: An estimated $11 \%$ of the female population in the United States will undergo surgery for prolapse or stress incontinence by the age of 80 . Repair with vaginal graft augmentation was offered in hopes of reducing the recurrence rate and need for reoperation. Concerns about the use of transvaginal mesh appeared in 2008, and in 2011 the FDA concluded that, "serious adverse events associated with mesh use are not rare". This action resulted not only in a significant amount of legal ramifications but changed the way many surgeons address prolapse and counsel their patients. Given this, we hypothesize that the FDA Notification is likely to have a significant impact on training of urogynecology fellows. The increased number of cases dedicated to mesh removals and revisions has come at the expense of other "bread and butter" urogynecology procedures such as prolapse and incontinence surgeries.

Objectives: We sought to determine the impact of the transvaginal mesh FDA Public Health Notification on the number and types of procedures performed by fellows in a Female Pelvic Medicine and Reconstructive Surgery Fellowship at a single academic center.

Methods: De-identified surgical case logs were examined to determine the procedures performed during the 18 months prior to the FDA warning compared to the 18 months following this warning (January 1, 2010-January 1, 2013) at a single academic center. Data were analyzed using chi-square. $\mathrm{P}<0.05$ was considered significant.

Results: During the study period, a total of 1511 surgeries were performed by fellows. After the FDA notification, the total number of mesh-related cases (including mesh revisions and removals) significantly increased ( $13.1 \%$ vs. $20.2 \%, p<0.001)$. Likewise, the number of surgical cases to address pain increased $(4.9 \%$ vs. $11.6 \%, p<0.001)$. This has come at the expense of prolapse and incontinence surgeries. Prior to the FDA notification, $41.8 \%$ of surgeries performed were incontinence cases compared to $33.5 \%$ after the notification ( $p$ $=0.001$ ). Similarly, the total number of prolapse surgeries dropped from $60.8 \%$ to $54.7 \%(p=0.017)$. Furthermore, the number of procedures in which vaginal mesh was used to treat prolapse has significantly decreased by approximately $50 \%$ (10.6\% vs. $4.7 \% \mathrm{p}=0<.001$ ).

Conclusion: The FDA notification has significantly impacted the number and type of cases fellows are exposed to during training in a FPMRS program.

Clinical Relevance: Female Urology-including Incontinence

\section{Podium \#45}

THE UTILIZATION OF MESH IN PROLAPSE AND INCONTINENCE SURGERY BEFORE AND AFTER THE 2008 FDA PUBLIC HEALTH NOTIFICATION IN A PRIVATE INSURER POPULATION

Jamal Nabhani, MD, David Ginsberg, MD

USC Institute of Urology, Los Angeles, CA

(Presented by: Jamal Nabhani, MD)

Introduction: The FDA public health notification (PHN) on transvaginal surgical mesh in October 2008 and subsequent update in July 2011 has prompted concerns from patient and provider alike about the use of graft material for the treatment of pelvic organ prolapse (POP) and stress urinary incontinence
(SUI). There has been limited investigation into the effect of these warnings on the surgical treatment of POP and SUI in the ensuing time.

Methods: Claims from the Humana administrative database (5.2 million patients, 52\% female with average age of 48 years, 307 million observed procedures) were queried using current procedural terminology (CPT) and international classification of disease, 9th Revision (ICD-9) codes for POP and/or SUI between January 2007 and November 2012, a time period spanning before, between, and after the PHN and update.

Results: The query identified 69,786 distinct procedures for SUI and/or POP over the nearly 6 year period, of which 29,946 (42.9\%) involved implantation or removal of mesh and 39,840 (57.1\%) which did not. In $2008,46.3 \%$ of procedures for POP or SUI involved mesh whereas the use of mesh declined to $35.2 \%$ in $2012(\mathrm{P}<1.8 \times 10-7)$. The main driver of this change was the decreased rate of POP repair with mesh from 24.1 (cases/month per 100,000 female beneficiaries) in 2008 to 18.9 in 2012 $(P=0.001)$. Over the same period, mesh removal/revision rates increased ( 2.4 to $5.7, \mathrm{P}=4.2 \times 10-5$ ). The rate of sling procedures between 2008 and 2012 did not significantly change (58.2 to $58.9, \mathrm{P}=0.46)$.

Conclusion: The FDA PMH has significantly decreased the use of surgical mesh in POP surgery although the overall incidence of surgical treatment for POP and SUI has increased. POP repair with mesh has significantly decreased while mesh removal/ revision makes up a larger portion of mesh procedures performed. The rate of sling procedures for SUI has been unchanged over the same period.

\section{POP and SUI Procedures}

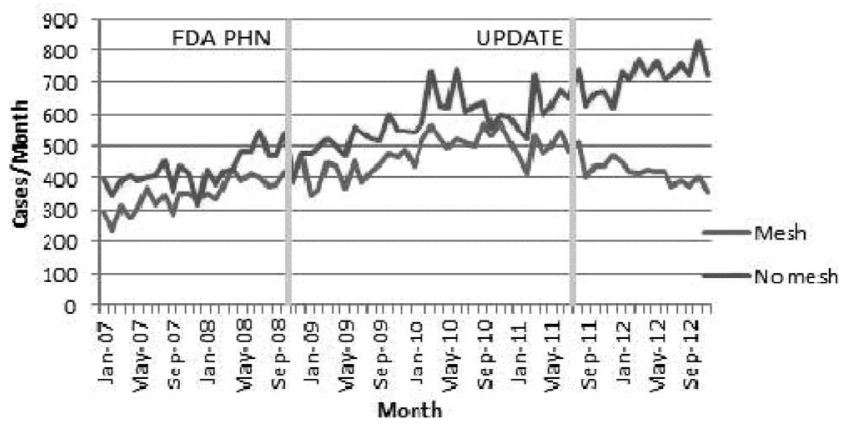

\section{Podium \#46}

\section{POSTOPERATIVE PAIN OUTCOMES AFTER TRANSVAGINAL MESH REVISION}

Jill Danford, MD, David Osborn, MD, Stephen Mock, MD, Xuechao Zhang, Vanessa Buie, W. Stuart Reynolds, MD, Daniel Biller, MD, Roger Dmochowski, MD

Nashville, TN

(Presented by: David Osborn, MD)

Objectives: Pelvic surgery divisions of tertiary care centers are seeing increasing numbers of patients with previous vaginal mesh placement for pelvic organ prolapse or urinary incontinence who desire mesh removal. Common complaints among these patients are dyspareunia, pelvic pain and bladder pain. The purpose of this study is to determine if surgical removal or revision of vaginal mesh improves patients' subjective complaints of pelvic pain associated with original placement of mesh.

Methods: A retrospective review of electronic medical records of female patients who underwent excision or revision of 
vaginal mesh in both Urology and Gynecology Departments at Vanderbilt University from January 2000 to August 2012 was performed. Patients were identified using associated CPT codes for mesh removal or revision and urethrolysis including patients who had previous sling procedures and/or vaginally placed mesh. Patient age, relevant medical history including menopause status, previous hysterectomy, smoking status, and presence of diabetes, fibromyalgia, interstitial cystitis and chronic pelvic pain were obtained. Patients' pre-operative and post-operative pain complaints at most recent post-operative visit were assessed. Patients were included in the study if their pain began after their original mesh surgery and the pain was related to the surgery.

Results: Of the 481 patients who underwent surgery for mesh revision, removal or urethrolysis, 229 patients met our inclusion criteria. Mean age was 54 years old. One hundred sixty nine patients (73\%) reported that their pain either improved or resolved, 19 (8\%) reported pain worsened and 45 (19\%) reported that pain remained unchanged after surgery. Prior history of chronic pelvic pain was associated with increased risk of failure of the procedure to relieve pain (OR $0.289,95 \%$ CI $0.126-0.659 \mathrm{p} 0.003)$. Of the 131 patients who had mesh perforation or exposure, 103 had exposure in the vagina, 14 perforation into the bladder and 14 perforation into the urethra. This was not associated with worse outcome in terms of pain relief. Median follow-up was 12.4 months (IOR 13).

Conclusion: Excision or revision of vaginal mesh appears to be effective in improving patients' pain symptoms the majority of the time. Patients with a history of chronic pelvic pain are at an increased risk of no improvement or of worsening of their pain. Mesh erosion into vagina, bladder or urethra is not associated with a worse outcome.

Clinical Relevance: IC and Pelvic Pain-UTI / Inflammatory

\section{Podium \#47}

\section{DE-NOVO VOIDING DYSFUNCTION IN WOMEN AFTER RADICAL GYNECOLOGICAL SURGERY}

\author{
Karl Coutinho, $\mathrm{MD}^{1}$, Steven Weissbart, $\mathrm{MD}^{1}$, \\ Donghua Xie, $\mathbf{M D}^{2}$ and Jaspreet Sandhu, $\mathbf{M D}^{2}$ \\ ${ }^{1}$ Mount Sinai Hospital, New York, NY; ${ }^{2}$ Memorial Sloan \\ Kettering Cancer Center, New York, NY \\ (Presented by: Karl Coutinho, MD)
}

Introduction: Voiding dysfunction in women who underwent radical gynecological surgery is poorly understood. In this study, we assess and characterize the urodynamic abnormalities of women with de-novo voiding dysfunction after a radical pelvic operation.

Material and Methods: After IRB approval, women who underwent radical gynecological surgery and underwent urodynamics testing between Jan 2003-Jan 2013 were identified. After excluding women with urological procedures or complaints prior to their radical pelvic surgery, a total of 141 records were analyzed. Type of surgery, neoplasm/disease histology, operative technique, findings, operative time, surgical blood loss, use of chemotherapy, use of radiotherapy, and video-urodynamic data were analyzed.

Results: Mean patient age was 53 years old (range 19-76) at the time of their GYN surgery and time to urologic referral was 23 months. Indications for surgery were ovarian cancer (28.5\%), endometrial cancer (22.1\%), cervical cancer (15.7\%), and benign disease (11.4\%). Most (93\%) underwent a hysterectomy and bilateral salpingo-oophorectomy (BSO), 65\% underwent a pelvic or para-aortic lymph node dissection, and $40 \%$ under- went tumor debulking. Neoadjuvant and adjuvant chemotherapy was utilized in $14.9 \%$ and $53.2 \%$ of patient's respectively. Neoadjuvant and adjuvant radiation therapy was utilized in $4.2 \%$ and $29.0 \%$ of patients respectively. Chief complaints at the time of referral included urinary incontinence (74.5\%), urgency (39.7\%), incomplete emptying (16.3\%), retention (12.1\%), and prolapse (5.7)\%. On VUDS, $7.1 \%$ had detrusor overactivity, $4.3 \%$ had detrusor overactivty incontinence, $32.6 \%$ had stress incontinence, $54.6 \%$ had detrusor underactivity, 3.5\% had impaired compliance, and 5.0\% had bladder outlet obstruction. Mean cystometric capacity was $373.9 \mathrm{ml}$, mean Omax was $8.0 \mathrm{ml} / \mathrm{sec}$ and mean Pdet Omax was $20.9 \mathrm{~cm} \mathrm{H2O}$. On multivariate analysis no operative or disease factors were associated with the development of stress incontinence, detrusor overactivity or detrusor underactivity.

Conclusion: The nature of de-novo voiding dysfunction in women which develops subsequent to radical gynecological surgery is varied. As operative and disease factors were not predictive of the type of voiding dysfunction, urodynamic studies will continue to play an important role in the diagnosis and subsequent management of voiding dysfunction which develops after radical gynecological surgery.

Clinical Relevance: Urodynamics

\section{Podium \#48}

SUPERIORITY OF FESOTERODINE 8 MG VERSUS FESOTERODINE 4 MG IN REDUCING URGENCY URINARY INCONTINENCE EPISODES IN SUBJECTS WITH OVERACTIVE BLADDER: RESULTS: OF THE RANDOMIZED, DOUBLE-BLIND, PLACEBO-CONTROLLED EIGHT TRIAL

Christopher Chapple ${ }^{1}$, Tim Schneider, $\mathbf{M D}^{2}$, François Haab, $\mathrm{MD}^{3}$, Franklin Sun, $\mathrm{MS}^{4}$, Laurence Whelan, $\mathrm{PhD}^{5}$, David Scholfield, $\mathrm{PhD}^{5}$, Erika Dragon, $\mathrm{MD}^{6}$ and Erin Mangan, $\mathrm{PhD}^{4}$

${ }^{1}$ The Royal Hallamshire Hospital; ${ }^{2}$ Praxisklinik Urologie Rhein/ Ruhr; ${ }^{3}$ Hopital Tenon; ${ }^{4}$ Pfizer Inc; ${ }^{5}$ Pfizer Ltd; ${ }^{6}$ Pfizer PIO

(Presented by: Christopher Chapple)

Introduction and Objectives: It is assumed when drugs have two doses, the higher dose provides increased efficacy. To date there is a lack of evidence in the antimuscarinic class that higher doses are more efficacious. In the EIGHT (Evaluation of urinary urge Incontinence patients Given fesoterodine $8 \mathrm{mg}$ vs fesoterodine $4 \mathrm{mg}$ in a Head-to-head efficacy Trial) trial we assessed the superiority of fesoterodine (FESO) 8mg vs FESO $4 \mathrm{mg}$ and placebo (PBO).

Methods: This was a 12-week, randomized, double-blind, PBOcontrolled trial. Eligible subjects aged $\geq 18$ y with overactive bladder (OAB) symptoms for $\geq 6$ months, $\geq 8$ micturitions and $\geq 2$ and $\leq 15$ urgency urinary incontinence (UUI) episodes $/ 24 \mathrm{~h}$ on baseline diary, and at least moderate bladder-related problems on the Patient Perception of Bladder Condition (PPBC) were randomized to treatment with FESO $8 \mathrm{mg}$, FESO $4 \mathrm{mg}$, or $\mathrm{PBO}$ once daily. Subjects randomized to FESO $8 \mathrm{mg}$ started with $4 \mathrm{mg}$ of FESO for 1 week, then increased to $8 \mathrm{mg}$ for 11 weeks. Subjects completed bladder diaries the PPBC, Urgency Perception Scale (UPS), and Overactive Bladder Questionnaire (OAB-q) at baseline and week 12 . The primary endpoint was change from baseline to week 12 in UUI episodes/24 h for FESO $8 \mathrm{mg}$ vs FESO $4 \mathrm{mg}$ and PBO. The primary comparison (FESO $8 \mathrm{mg}$ versus $4 \mathrm{mg}$ ) was performed only if there was a statistically significant difference for $8 \mathrm{mg}$ vs PBO.

Results: At week 12, the FESO 8mg group $(n=779)$ had significantly greater improvements than the FESO $4 \mathrm{mg}$ ( $\mathrm{n}$ 
$=790)$ or PBO $(n=386)$ groups in UUI episodes, micturitions, and urgency episodes and in scores on the PPBC, UPS, and all $O A B-q$ scales and domains; diary-rate was significantly higher in the FESO $8 \mathrm{mg}$ group vs the FESO $4 \mathrm{mg}$ and $\mathrm{PBO}$ groups (all $\mathrm{P}<0.05$; Table). In the PBO, FESO $4 \mathrm{mg}$, and FESO $8 \mathrm{mg}$ groups, rates of dry mouth and constipation were $3 \%, 13 \%$, and $26 \%$ and $2 \%, 2 \%$, and $4 \%$, respectively; most cases were mild or moderate in all groups.

Conclusion: The EIGHT study demonstrates the superior efficacy of FESO 8mg for improving OAB symptoms, including UUI, and patient-reported outcomes vs FESO $4 \mathrm{mg}$ and PBO. These data support the benefit of two FESO doses for patients in clinical practice, some of whom require a higher dose to achieve optimal symptom relief.

clinical Relevance: Male Incontinence

\begin{tabular}{|c|c|c|c|}
\hline Outcome & $\begin{array}{c}\text { PBO } \\
(n=386)\end{array}$ & $\begin{array}{c}\text { FESO } 4 \mathrm{mg} \\
(\mathrm{n}=790)\end{array}$ & $\begin{array}{c}\text { FESO } 8 \mathrm{mg} \\
(\mathrm{n}=779)\end{array}$ \\
\hline \multicolumn{4}{|c|}{ Dary variables; LS mean change from baseline } \\
\hline UUl episodes & -2.2 & $-29^{\circ}$ & -3.1 t \\
\hline Micturitions & -1.6 & $-2.5^{\circ}$ & $-3.0 *$ \\
\hline Urgenoy episodes & -3.0 & $-4.2^{*}$ & $-50^{-1}$ \\
\hline Diary-dy rate, os & 39.5 & $49.2^{*}$ & $57.8^{-4}$ \\
\hline \multicolumn{4}{|l|}{ PPBC score, \%" } \\
\hline 2-point improvement & 276 & $40.0^{\circ}$ & $486^{\circ t}$ \\
\hline 1-point improvement & 302 & 30.0 & 25.1 \\
\hline No change. & 35.1 & 24.6 & 22.3 \\
\hline Deterioration & 7.2 & 5.4 & 4.0 \\
\hline \multicolumn{4}{|l|}{ UPS soore, of } \\
\hline Improvement & 37.9 & 43.1 & $50.5^{\text {th }}$ \\
\hline No change & 55.5 & 51.6 & 45.1 \\
\hline Deterioration & 66 & 5.3 & 4.4 \\
\hline \multicolumn{4}{|c|}{ OAB- q scores, LS mean change from baseline } \\
\hline Symptom Bother & 19 & 24 & $28^{* 4}$ \\
\hline Tolal HRQL & -22 & -30 & $-35+t$ \\
\hline
\end{tabular}

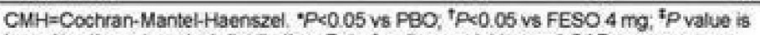
based on the categorical distribution. Data for diary variables and $O A B-q$ scores were. based on the categorical distribution. Data for diary variables and OAB-q scores were arssessed using analysis of covariance model with terms for treatment, country, and baseline
value as a covariate; models for UUI and OAB-q included baseline by treatment interaetor, value as a eovariate; models for UUI and OAB-q included baseline by treatment interaeton,
which was quartitative. Dary-dry rate was analyzed using a CMH test controling for country. Which was quarditalive. Dary-dy rate was anslyzed using a CMH test controling for country. for country.

\section{Podium \#49}

CONTEMPORARY COMPARISON BETWEEN RETROPUBIC MID URETHRAL SLING (MUS) AND AUTOLOGOUS PUBOVAGINAL SLING (PVS) FOR STRESS URINARY INCONTINENCE (SUI) AFTER THE FDA ADVISORY WARNING

Stephen Mock, $\mathrm{MD}^{1}$, Jonathan Angelle ${ }^{2}$, W. Stuart Reynolds, $M^{1}$, David J. Osborn, $M^{1}$, Roger R. Dmochowski, $M^{1}$ and Alexander Gomelsky, $\mathbf{M D}^{2}$

${ }^{1}$ Vanderbilt University, Nashville, $\mathrm{TN} ;{ }^{2}$ Louisiana State University, Shreveport, LA

(Presented by: Stephen Mock, MD)

Introduction and Objectives: At present, synthetic MUS is widely considered to be the standard of care for female SUI and has largely replaced the PVS, a traditionally more morbid procedure. In light of the 2011 FDA warning on mesh use for pelvic organ prolapse (POP), concerns regarding mesh use, in general, have been raised by both patients and providers. As a result, fewer patients may be opting for mesh. The aim of this study is to compare efficacy and complication rates in a cohort of women who were offered either procedure after the FDA warning and made an informed decision on procedure option. Methods: A total of 201 women underwent either PVS or MUS after the FDA warning was issued in July 2011. Exclusion criteria included prior anti-incontinence surgery, concomitant surgery other than hysterectomy, and POP stage $>2$. Patients were followed prospectively with validated quality of life (OoL) questionnaires and subjective SEAPI (stress, emptying, anatomy, protection, inhibition) scores. Treatment success was defined as absence of subjective or objective SUI. Peri-operative complications were classified via the Clavien-Dindo scale.

Results: Minimum follow-up was 12 months (mean 14.6). Ninety one (45\%) and 110 (55\%) women opted for PVS and MUS, respectively. Preoperative demographics and clinical parameters, including age, body mass index, parity, pad usage, mean abdominal leak point pressure, severity of concomitant POP, SEAPI scores, and mean OoL indices did not differ significantly between the groups. There was no difference in the proportion of women undergoing concomitant hysterectomy (52.7\% vs. $50 \%, p=0.7)$. Treatment success was similar (75.8\% vs. $80.9 \%$, $\mathrm{p}=0.38)$ and there was no difference in rates of postoperative voiding dysfunction ( $1.1 \%$ vs. $1.8 \%, p=0.68)$ or de novo urge incontinence ( $4.4 \%$ vs. $4.5 \%, p=0.96)$. Length of hospitalization did not significantly differ ( 1.18 vs. 1.16 days, $p=0.9$ ). There was no difference in the occurrence of Clavien-Dindo surgical complications between the two groups (6.6\% vs. $10 \%, p=0.39$ ). Conclusion: Since the 2011 FDA warning, more women are forgoing the use of mesh in the treatment of SUI. In a contemporary cohort of women considered suitable candidates for either sling, both procedures offer comparable efficacy and complication rates. PVS may be safely offered to patients who would otherwise be good candidates for MUS if they are concerned with the implantation of mesh.

Source of Funding: None

Clinical Relevance: Female Urology-including Incontinence

\section{BASIC SCIENCE POSTERS}

\section{Poster \# BS1}

\section{NEUROMUSCULAR NICOTINIC RECEPTORS MEDIATE BLADDER CONTRACTIONS FOLLOWING BLADDER REINNERVATION WITH SOMATIC TO AUTONOMIC NERVE TRANSFER AFTER DENERVATION BY SPINAL ROOT TRANSECTION}

Sandra M. Gomez Amaya, DVM ${ }^{1}$, Mary F. Barbe, $\mathrm{PhD}^{1}$, Justin M. Brown, $\mathrm{MD}^{2}$, Alan S. Braverman, $\mathrm{PhD}^{1}$, Neil S. Lamarre, $\mathrm{PhD}^{1}$ and Michael R. Ruggieri, $\mathrm{PhD}^{3}$

${ }^{1}$ Temple University School of Medicine, Department of Anatomy and Cell Biology. Philadelphia, PA; ${ }^{2}$ University of California San Diego; ${ }^{3}$ Temple University School of Medicine, Department of Anatomy and Cell Biology. Philadelphia, PA and Shriners Hospital of Philadelphia, Pennsylvania

(Presented by: Sandra M Gomez Amaya, DVM)

Introduction: This study investigates whether the reinnervated neuronal pathway mediates contraction via the same neurotransmitter and receptor mechanisms as the original pathway.

Methods: Following bladder denervation by transection of sacral roots peripheral nerve transfer was done with bilateral genitofemoral to pelvic nerve transfer (GFNT) and unilateral (left) femoral nerve to bilateral pelvic nerve transfer (FNT). Reinnervation was assessed 8-10 months post operatively by monitoring bladder pressure during electrical stimulation of the transferred nerves, spinal roots, and spinal cord segments as well as retrograde neurotracing of fluorogold injected into the bladder 3 weeks before euthanasia. In 5 denervated control dogs, bladder pressure was induced by stimulation of the pelvic plexus but not the sacral spinal cord.

Results: Of the 17 animals with GFNT, 14 (82\%) demonstrated functional bladder reinnervation as evidenced by increased bladder pressure during stimulation of the transferred GFN or the L3 or L4 spinal roots. This was observed bilaterally in 4 dogs, and unilaterally in 10. Lumbar spinal cord stimulation caused 
increased bladder pressure in 9 of 10 (90\%) animals with FNT. In normal control animals (either non-operated or sham operated) succinyl choline had no effect on bladder pressure but substantially reduced urethra and anal sphincter pressure induced by stimulation of either the lumbosacral spinal cord or the $52-3$ spinal root. In animals with reinnervation by GFNT and FNT, bladder pressure increases induced by stimulation of the transferred somatic nerves or the lumbar spinal segments that contribute axons to GFN and FN (GFN:L2-4, FN: L1-4\&L6), were completely blocked by succinyl choline. In denervated control $(\mathrm{N}=5)$ and GFNT $(\mathrm{N}=5)$ dogs, few to no fluorogoldlabeled cell bodies were detected in lower lumbar and sacral segments, indicating successful denervation. In sham operated $(\mathrm{N}=3)$ and unoperated controls $(\mathrm{N}=3)$, fluorogold-labeled cell bodies were observed in L7, S1, S2 and S3 cord segments. In the GFNT dogs, fluorogold-labeled neuronal cell bodies were observed in ventral horns that contribute to the GF nerve (L3-L4).

Conclusion: These data suggest that succinyl choline sensitive nicotinic receptors that normally mediate only skeletal muscle neurotransmission appear in the new neuronal pathway to the detrusor muscle after GFNT or FNT. This suggests neuroplasticity in the end organ after reinnervation by somatic motor axons.

Clinical Relevance: Neurogenic Bladder

\section{Poster \# BS2}

\section{INCREASED PROSTATE CONTRACTIONS IN CHRONIC} ISCHEMIA: THE ROLE OF OXIDATIVE STRESS

Kazem Azadzoi, $\mathrm{MD}^{1}$, Oi Zhang, $\mathrm{PhD}^{2}$ and Mike Siroky, $\mathrm{MD}^{1}$

${ }^{1}$ VA Boston \& Boston University; ${ }^{2}$ VA Boston, Boston, MA

(Presented by: Kazem Azadzoi, MD)

Introduction and Objectives: Clinical studies have suggested a close correlation between decreased prostate blood flow and lower urinary tract symptoms in the elderly patients. Our goal was to examine the effects of chronic ischemia on prostate smooth muscle contractions in an animal model and characterize human prostatic smooth muscle cells (SMC), epithelial cells (EC), and stromal cells (SC) reactions to hypoxia and oxidative stress conditions.

Methods: A rabbit model of chronic prostate ischemia was developed by creating aorto-iliac arterial atherosclerosis. Prostate tissue contractility was examined in the organ bath. Confluent cultured human prostate primary SMC, EC, and SC were incubated under normoxia, continuous hypoxia and oxidative stress conditions for 48 hours using a computerized servo-control cell oxycycler system then collected and processed for analysis. Oxidative stress markers in the rabbit prostate tissue and cultured cells were examined using fluorometric analysis and enzyme immunoassay.

Results: Moderate ischemia significantly increased rabbit prostate smooth muscle contractions to electrical field stimulation and led to accumulation of advanced oxidation protein products and malondialdehyde, suggesting oxidative stress. Increased prostate tissue contractions were reversed by treatment with antioxidants. In cell studies, lipid peroxidation was found in SMC hypoxia and in SMC, EC and SC oxidative stress. Protein oxidation took place in EC hypoxia and in SMC, EC and SC oxidative stress. The levels of oxidative and nitrosative products increased in SMC, EC and SC oxidative stress. Antioxidant capacity of SMC, EC and SC decreased in both hypoxia and oxidative stress conditions. All reactions of the prostate SMC, EC and SC to hypoxia and oxidative stress were associated with marked DNA damage.

Conclusion: The mechanism of increased prostate tissue contractions in ischemia appears to involve oxidative modifications of cellular and subcellular elements. Human prostate SMC, EC and SC were highly sensitive to hypoxic and oxidative stress conditions exhibiting differential reactions. DNA damage was a widespread phenomenon in prostate SMC, EC and SC after exposure to hypoxia and oxidative stress. Increased prostate contractions in ischemia and oxidative stress appeared to involve free radical incursion of SMC, EC, and SC, impairment of cellular antioxidant defense system and subsequent accumulation of oxidative and nitrosative cytotoxic elements.

Clinical Relevance: LUTS / Voiding Dysfunction

\section{Poster \# BS3}

CORRECTION OF HYPERGLYCEMIA AND HYPERINSULINEMIA BY GENETIC MODIFICATION RESTORES BLADDER DYSFUNCTION ASSOCIATED WITH TYPE 2 DIABETES

Zongwei Wang, $\mathrm{PhD}^{1}$, Vivian Cristofaro, $\mathrm{PhD}^{2}$, Hongying Cao, $\mathrm{PhD}^{2}$, Evgeniy Kreydin, $\mathrm{MD}^{1}$, Joseph Gabrielsen, $\mathrm{MD}, \mathrm{PhD}^{1}$, Rongbin $\mathrm{Ge}, \mathrm{MD}, \mathrm{PhD}^{1}$, Maryrose Sullivan, $\mathrm{PhD}^{2}$ and Aria Olumi, $\mathbf{M D}^{1}$

${ }^{1}$ Massachusetts General Hospital, Harvard Medical School; ${ }^{2}$ VA Boston Healthcare System, Harvard Medical School

(Presented by: Zongwei Wang, PhD)

Introduction and Objectives: Diabetes bladder dysfunction (DBD) is a major urologic complication associated with type 2 diabetes (DM2). In a mouse model that develops DM2 using a hepatic-specific insulin receptor substrate 1 and 2 (Irs1/Irs2) deletions (double knockout: DKO), we have shown that DBD occurs progressively with hyperactivity in the early stage and hypoactivity in late stage of DBD. Here we demonstrate that correction of hyperglycemia/hyperinsulinemia with a third hepatic-specific deletion of Foxo1 gene (i.e.: IRS1/IRS2/Foxo1 triple knockout: TKO) restores the bladder dysfunction in DKO type 2 diabetic animals.

Materials \& Methods: Insulin and glucose levels were measured and glucose tolerance tests (GTT) were carried out in DKO, TKO and control animals at 4, 8, 12, and 20 weeks of age. Bladder functional alterations were evaluated by in vivo cystometry and voiding stain on paper (VSOP) for 12 and 20 week old mice. Bladders were harvested for ex vivo muscle strip contraction analyses.

Results: The DKO mice developed significant insulin resistance and glucose tolerance starting from 5 weeks of age, and persisted at the age of 8,12 , and 20 weeks. TKO animals with hepatic-specific deletion of Irs1/Irs2/Foxo1 genes did not develop hyperglycemia or hyperinsulinemia. In the diabetic DKO animals, the post void residual (PVR) urine volume in invivo cystometry was elevated and the voiding efficiency was lowered at 12 weeks and 20 weeks in comparison to the controls. The bladder compliance and capacity of 12 week but not 20 week DKO mice was lower than age-matched controls. An abnormal voiding pattern was observed in the DKO mice as determined by VSOP analysis with lower micturition volumes, and higher frequency of small volume voids. In TKO animal, the voiding parameters were restored to normal levels equivalent to age-matched controls.

Conclusions: Our findings demonstrate that secondary complication of diabetes, DBD, is corrected in diabetic animals with 
hepatic-specific genetic modification in the TKO mice, with deletions of IRS1/IRS2 \& Foxo1 genes. Our findings suggest that the DKO and TKO reversible diabetic mouse model system is a rational model to evaluate the pathophysiology and molecular alterations associated with diabetic cystopathy.

Funding: NIH/NIDDK grant (Animal Models of Diabetic Complications Pilot \& Feasibility Project, 09MCG72) and the American Urological Association/Pfizer Pharmaceutical Company competitive grants to AFO.

Clinical Relevance: LUTS / Voiding Dysfunction

\section{Poster \# BS4}

\section{EVIDENCE OF NEUROGENIC BLADDER DYSFUNCTION IN A MOUSE MODEL OF MULTIPLE SCLEROSIS}

Neil Lamarre, $\mathrm{PhD}^{1}$, Alan Braverman, $\mathrm{PhD}^{1}$, Anna Malykhina, $\mathrm{PhD}^{2}$ and Michael Ruggieri, $\mathrm{PhD}^{1}$

${ }^{1}$ Temple University School of Medicine, Philadelphia, PA;

${ }^{2}$ University of Pennsylvania, Philadelphia, PA

(Presented by: Neil Lamarre, PhD)

Introduction: Patients with neurodegenerative diseases such as multiple sclerosis, Parkinson's, Alzheimer's and ALS often present with lower urinary tract symptoms (urinary frequency, urgency, nocturia, retention) resulting from damage to the peripheral and central nervous systems. These studies were designed to examine the underlying changes that may be common to neurogenic bladder dysfunction using a mouse model of demyelination.

Methods: Bladders from 12-week-old male C57BL/6J mice with coronavirus-induced encephalomyelitis (CIE, a chronic, progressive demyelinating disease model of human MS), and agematched controls, were cut into 5-7 strips and suspended in physiological muscle baths for tension measurement in response to agonists and electric field stimulation (EFS). In some strips, the mucosa was first removed.

Results: The maximum effect of EFS was not significantly different between CIE and normal mice. Nerve-evoked EFS contractions (tetrodotoxin sensitive) were blocked by a combination of atropine (cholinergic antagonist) and alpha, beta-methylene ATP (an ATP analog that desensitizes purinergic receptors). In response to EFS, the alpha, beta-methylene ATP-resistant (cholinergic) component of contraction was significantly reduced in CIE mice, while the atropine-resistant (purinergic) component increased. Removal of mucosal (urothelial) layer in CIE mice restored the cholinergic component. Co-incubation of mucosa from CIE mice with normal mouse bladder strips for 3 hours suppressed nerve-evoked cholinergic contractions.

Conclusions: Previous data from our lab has shown that soluble factors released from the urothelium can influence the functional properties of the underlying smooth muscle. These data suggest a deficit in nerve-evoked cholinergic contraction that is not due to the ability of the smooth muscle to respond to acetylcholine. We conclude that neurodegenerative bladder dysfunction in this model of multiple sclerosis may be due in part to pathologic changes in the urothelium that, through a diffusible factor, cause suppression of muscarinic receptormediated contractile response and augmentation of the purinergic response of the underlying muscle. This finding in an animal model is consistent with previously reports of increased purinergic contractions in neuropathic human bladder.

Clinical Relevance: Neurogenic Bladder

\section{Poster \# BS5}

\section{CHARACTERIZATION OF FUNCTIONAL BLADDER} REGENERATION IN MICE

Mona Zarifpour, PharmD ${ }^{1}$, Jason Sandberg, $\mathbf{M D}^{1,2}$, Mehran Abolbashari, $\mathbf{M D}^{1}$, Manasi Vadhavkar, $\mathbf{M S}^{1}$, Sneha Kelkar, $\mathrm{PhD}^{1}$, Aaron Mohs, $\mathrm{PhD}^{1,3}$, Frank Marini, $\mathrm{PhD}^{1}$, Karl-Erik Andersson, $\mathrm{MD}, \mathrm{PhD}^{1}$ and George Christ, $\mathrm{PhD}^{1,3}$

${ }^{1}$ Wake Forest Institute for Regenerative Medicine, WinstonSalem, NC; ${ }^{2}$ Department of Urology, Wake Forest Baptist Medical Center, Winston-Salem, NC; ${ }^{3}$ Wake Forest - Virginia Tech School of Biomedical Engineering and Sciences, WinstonSalem, NC

(Presented by: Jason Sandberg, MD)

Introduction and Objectives: Subtotal Cystectomy (STC; surgical removal of $50-60 \%$ of the bladder) in rodents is known to elicit a robust proliferative response with the capacity to regenerate a fully functioning bladder within 8-12 weeks. Previous studies have established this surgical procedure as a model to gain insight into the normal regenerative process in adult rats. The goal of this study was to characterize the same responses that mediate this phenomenon in mice, a species that can be genetically engineered to investigate a diverse array of molecular alterations on functional bladder regeneration, including specific cell-based fluorescence imaging technologies.

Methods: Female C57 black mice underwent STC, and at 4 and 12 weeks post-STC, bladder regeneration was assessed via cystometry and ex vivo pharmacologic organ bath studies. Histology was also performed to measure bladder wall thickness and the composition/architecture of the bladder wall.

Results: We observed a time-dependent increase in bladder capacity following STC, such that 12 weeks post-STC, the sizes of regenerated bladders and micturition volumes were indistinguishable from those of age-matched controls (Fig. 1A, C). Bladders emptied completely at all time points studied (i.e., no increases in residual volume), consistent with functional bladder regeneration. There were no significant differences in bladder wall thickness (Fig. 1B) from controls, or in the percentage of smooth muscle in the detrusor layer (not shown). The maximal contractile response to pharmacological activation and electrical field stimulation increased over time in isolated tissue strips from regenerating bladders, but remained lower at all time points compared with controls.

Conclusions: This study extends our investigation of mammalian bladder regeneration from rats to mice. Establishing a validated model for the study of de novo organ regeneration in mice will allow for further characterization of this phenomenon through detailed investigation of its molecular and cellular basis.

Funding: NIH P20 Grant DK097806

Clinical Relevance: Urodynamics

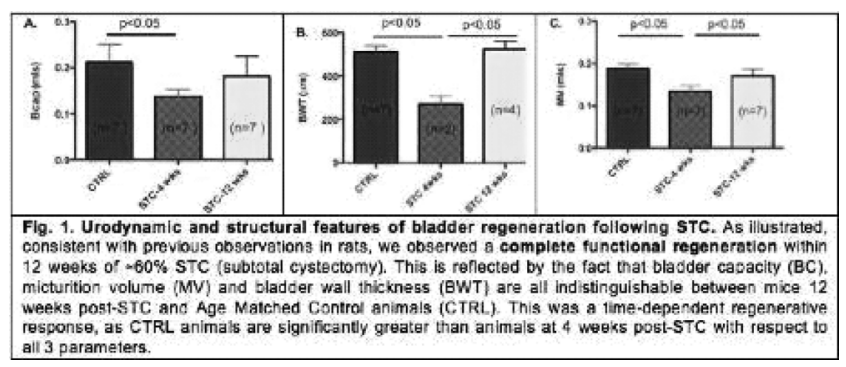




\section{Poster \# BS6}

OVEREXPRESSION OF TNF IN MOUSE UROTHELIUM MINICS INTERSTITIAL CYSTITIS DYSFUNCTION

Wenbin Yang, PhD, Ryan Yaggie, BS, David Klumpp, PhD Northwestern University

(Presented by: Wenbin Yang, PhD)

Introduction: Interstitial cystitis/bladder pain symptom (IC/ BPS), a chronic bladder condition that is often regarded as a neurogenic cystitis. Patient symptoms are correlated with lamina propria mast cells and urothelial lesions. We previously characterized a murine neurogenic cystitis model that recapitulates mast cell accumulation and urothelial lesions, and these events were dependent upon TNF. To further explore the role of TNF in bladder inflammation and function, we generated a transgenic mouse model of chronic TNF overexpression in urothelium.

Methods: We generated transgenic mice with TNF under the control of the uroplakin II (UPII) promoter. Transgenic mouse lines were maintained by backcross onto wild-type C57BL/6J mice, and evaluated for urinary function, urothelial lesions and pelvic tactile allodynia as a measure of visceral pain.

Results: TNF mRNA and protein were expressed at greater levels in bladders of UPII-TNF mice than wild-type littermates. UPII-TNF mice showed significantly increased urinary frequency and decreased urine output per void when compared with wild-type littermates. UPII-TNF mice had increased urothelial apoptosis and loss of urothelial integrity consistent with urothelial lesions. Overexpression of TNF was also associated pelvic tactile allodynia relative to the wild-type mice.

Conclusions: UPII-TNF mice with bladder-specific TNF overexpression display significant voiding dysfunction, urothelial lesions, and pelvic pain. Thus UPII-TNF mice are a novel model for characterizing mechanisms of IC symptoms and evaluating therapies.

Funding: NIH/NIDDK R01 DK066112 (DJK)

Clinical Relevance: Female Urology - including Incontinence

\section{Poster \# BS7}

\section{IS LOSS OF BLADDER VOLUME SENSITIVITY WITH AGING PRIMARY OR COMPENSATORY?}

Phillip Smith, $\mathrm{MD}^{1}$, Anthony DeAngelis, $\mathrm{PhD}^{2}$ and George Kuchel, $\mathrm{MD}^{3}$

${ }^{1}$ Department of Surgery, Center on Aging, University of Connecticut Health Center, Farmington CT; ${ }^{2}$ University of Connecticut Health Center; ${ }^{3}$ Center on Aging, University of Connecticut Health Center

(Presented by: Phillip Smith, MD)

Introduction: We have shown that bladder filling is altered but detrusor voiding contractility is preserved with aging in a mouse model (AJP 302; R577, 2012). Higher volume with similar pressure thresholds for voiding with aging suggest decreased volume sensitivity. We hypothesized that increased bladder volumes are compensatory for altered detrusor smooth muscle function, rather than a primary aging change.

Methods: Pressure-flow cystometry and bladder strip tensionlength studies were performed in 7 Mature (9-12 months) and 5 Old (22-24 month) female B6 mice. Three voids for each mouse were analyzed and mean per-mouse values calculated. Transverse mid-bladder rings were mounted in a myograph and passive and total tension in response to $\mathrm{KCl}$ stimulation were recorded at increasing lengths. Zero-tension length (Lo), maximum active (total-passive) tension (Tamax), and length at Tamax (Lmax) were determined. Group means were compared with t-test and correlations between CMG and in-vitro variables sought.

Results: Basal pressure was less in Old than in Mature mice (2.4 vs $4.3 \mathrm{~cm} . \mathrm{w}, \mathrm{p}=0.04)$, however voiding threshold, and maximum bladder pressures, compliance, voided volumes/flows, total and estimated detrusor pressure-time integral (PTI) for void and flow did not significantly differ between groups. Bladder and ring weights did not differ between groups. Other parameters including, Lo; the absolute and relative increase of Lmax over Lo; and Tamax did not significantly differ between groups, with and without correction for strip weights. Overall, basal and voiding threshold bladder pressures and compliance did not correlate with Tamax or Lmax. Some voiding variables significantly correlated $(r>0.5, p<0.05)$ with in-vitro measures. The voided volume $(r=0.59)$ and voiding PTI $(r=0.64)$ correlated with absolute increase in strip length over Lo, and voiding PTI with relative increase in length over Lo $(r=0.66)$. PTI during flow correlated with weight-adjusted Tamax $(\mathrm{r}=0.75)$ and negatively with Lo $(\mathrm{r}=-0.73)$. Voiding detrusor work correlated with weight-adjusted Tamax (0.63).

Conclusions: Preservation of voiding function in mid-life may be reflective of physiologic reserve in the setting of age-related declines, with decreased volume sensitivity rather than diminished detrusor function representing the primary underlying pathology associated with aging.

Funding: UConn Department of Surgery

Clinical Relevance: LUTS / Voiding Dysfunction

\section{Poster \# BS8}

\section{LONG-TERM EFFECTS OF SIMULATED CHILDBIRTH INJURY ON FUNCTION AND INNERVATION OF THE URETHRA}

Oi-Xiang Song, $\mathrm{MD}^{1,2,3}$, Brian Balog, $\mathrm{BS}^{1,3}$, James Kerns, $\mathrm{PhD}^{4}$, Dan Li Lin, $\mathrm{MD}^{1,3}$, Yinghao Sun, $\mathrm{MD}, \mathrm{PhD}^{2}$,

Margot Damaser, $\mathrm{PhD}^{1,3}$ and Hai-Hong Jiang, $\mathrm{MD}, \mathrm{PhD}^{1}$

${ }^{1}$ Department of Biomedical Engineering, The Cleveland Clinic,

Cleveland, $\mathrm{OH} ;{ }^{2}$ Department of Urology, Changhai Hospital, the Second Military Medical University, Shanghai, PR China; ${ }^{3}$ Louis Stokes Cleveland Veterans Affairs Medical Center, Cleveland, $\mathrm{OH} ;{ }^{4}$ Rush University Medical Center, Chicago, IL

(Presented by: Oi-Xiang Song, MD)

Introduction and Objectives: Pudendal nerve and external urethral sphincter (EUS) injury during vaginal delivery are risk factors for stress urinary incontinence (SUI). Although most patients with short-term postpartum SUI regain continence within 1 year, they have a higher predisposition to develop recurrent SUI years later, suggesting a possible mechanistic relationship. In contrast, animal models generally recover spontaneously and have not been studied much in the long term. The aim of this study was to investigate the long-term effects of simulated childbirth injury in rats.

Methods: Female Sprague-Dawley rats $(n=34)$ underwent sham injury or pudendal nerve crush and vaginal distension (PNC + VD), to simulate childbirth injury. Leak point pressure (LPP) and EUS electromyography (EMG) were recorded simultaneously 9 weeks later. The pudendal nerve $5 \mathrm{~mm}$ distal to the crush site, was procured qualitative histology. The urethra was harvested and sectioned for qualitative assessment of neuromuscular junctions (NMJs) via immunofluorescence. Acetylcholine receptors (AChRs) at motor end-plates were labeled with tetramethylrhodamine-conjugated $\alpha$-bungarotoxin and 
EUS muscle fibers were stained with Alexa Fluor conjugated phalloidin. Mouse anti-neurofilament $68 \mathrm{kDa}$ and $200 \mathrm{kDa}$ were used to label neurofilaments in innervating axons. A ttest was used to compare quantitative outcomes between groups, with $p<0.05$ indicating a significant difference.

Results: There was no significant difference in LPP or EUS EMG amplitude or firing rate between the PNC + VD and sham injury groups. The pudendal nerve motor branch distal to the injury site demonstrated splaying of regenerating axons coursing over the pudendal artery with no Wallerian degeneration. Nonetheless, 9 weeks after PNC + VD, EUS NMJs demonstrated tortuous innervating axons, consisting of multiple collateral fibers and diffusion of acetylcholine receptors at motor endplates, suggesting that muscle reinnervation was still ongoing. Conclusions: Although continence function was restored 9 weeks after simulated childbirth injury, innervation of EUS was not complete at this time point, suggesting that one of the long-term effects of simulated childbirth injury is persistent neurogenic deficiency. Insufficient neuromuscular recovery compounded by the effects of aging may lead to a delayed recurrence of SUI in this animal model. Future studies with longer outcome time points are warranted.

Funding: Dept. of Veterans Affairs and the Cleveland Clinic Clinical Relevance: Female Urology-including Incontinence

\section{Poster \# BS9}

\section{A NOVEL SURVIVAL MODEL OF PELVIC FLOOR DYSFUNCTION AFTER RABBIT PELVIC FLOOR AND TRANSVAGINAL ELECTRICAL STIMULATION}

Amy Dobberfuhl, $M^{1}$, Sara Spettel, $M^{1}$, Catherine Schuler ${ }^{2}$, Robert Levin, $\mathrm{PhD}^{2}$, Andrew Dubin, $\mathbf{M D}^{1}$ and Elise De, $\mathbf{M D}^{1}$ ${ }^{1}$ Albany Medical College, Albany, NY; ${ }^{2}$ Stratton VA Medical Center, Albany, NY

(Presented by: Amy Dobberfuhl, MD)

Introduction: Existing data supports a relationship between the levators and pelvic organ function; however there is a paucity of animal models for chronic pelvic floor dysfunction. We previously developed an acute rabbit model and demonstrated that direct needle stimulation of the pubococcygeous (PC) muscle resulted in cystometry (CMG) and electromyography (EMG) changes consistent with dysfunctional voiding; larger bladder capacity, longer interval between contractions and prolonged contraction duration. The current experiment explores the in vivo effects of needle and transvaginal stimulation using a survival model.

Methods: Twelve female adult virgin rabbits were housed in metabolic cages to record baseline voiding and defecation for 3 days. Anesthetized CMG/EMG was performed before and after treatment animals $(n=9)$ received bilateral tetanizing needle stimulation (4 trains $10 \mathrm{~s}$ apart, $15 \mathrm{~mA}, 25 \mathrm{~Hz}, 0.2 \mathrm{~ms}, 10$ pulses/ train) to the PC muscle and controls $(n=3)$ sham needle placement. After 7 days all animals were subjected to tetanizing transvaginal stimulation (5 minutes, $6.5 \mathrm{~mA}, 10 \mathrm{~Hz}$, $0.1 \mathrm{~ms}$ repetitive) and CMG/EMG. After 5 days a final CMG/EMG was performed.

Results: Mean fecal weight and urine production were similar between groups. At baseline, animals demonstrated heterogeneous voided volume and frequency behavior. Needle stimulation of the PC muscle significantly prolonged interval between CMG contractions with mean time to third contraction rising from 38 to 53 minutes ( $p=0.008$ vs. pre-stimulation), representing a significant mean increase of 15 minutes ( $p=0.022$ vs. 1 minute for control). Vaginal stimulation also significantly increased time to third contraction from 37 to 47 minutes ( $p=0.015$ vs. pre-stimulation). On linear regression of cage parameters, needle stimulation resulted in larger voided volumes and less frequent voids. Of rabbits that underwent needle stimulation, 7/9 (78\%) demonstrated voiding dysfunction vs. 6/12(50\%) after transvaginal stimulation.

Conclusion: Both direct PC and transvaginal electrical stimulation resulted in prolonged intervals between CMG contractions. Changes in cage parameters were primarily seen after direct needle stimulation, with larger volume and less frequent voids noted. Changes are consistent with dysfunctional voiding behavior, thus reiterating the central role of pelvic floor in coordinated voiding function.

Funding: Capital Region Medical Research Foundation \& Stratton VA Research and Development Office

Clinical Relevance: LUTS/Voiding Dysfunction

\section{Poster \# BS10}

\section{MIF IS ASSOCIATED WITH PREVENTION OF} OOPHORECTOMY-INDUCED EFFECTS ON BLADDER FILLING

Phillip Smith, $\mathrm{MD}^{1}$, Anthony DeAngelis, $\mathrm{PhD}^{2}$, Andrew Galffy, $\mathrm{MD}^{3}$ and George Kuchel, $\mathrm{MD}^{4}$

${ }^{1}$ Department of Surgery, Center on Aging, University of Connecticut Health Center, Farmington CT; ${ }^{2}$ University of Connecticut Health Center; ${ }^{3}$ Department of Ob/Gyn, University of Connecticut Health Center; ${ }^{4}$ Center on Aging, University of Connecticut Health Center

(Presented by: Phillip Smith, MD)

Introduction: Menopause and loss of estrogenization are associated with increasing prevalence of urinary symptoms in older women. MIF is a proinflammatory T-cell and macrophage-derived cytokine with neuroendocrine properties. Its pro-inflammatory activity is suppressed in the bladder by estrogen which inhibits MIF release from abundant urothelial stores. We hypothesized MIF contributes to oophorectomyinduced alterations in bladder function in the female mouse model.

Methods: Experiments were conducted using 2 mo female B6C57 mice by approved IACUC protocol. All mice had either oophorectomy (OVx) or a sham operation (Sham), in all cases 10 mice in each group. Cystometric studies were conducted using MIF +/+ (WT) and MIF -/- (KO) mice 3 months after sham or OVx surgery (WT Sham, WT OVx; KO Sham, KO OVx). In another protocol, a systemic MIF inhibitor (MI) was used. Cystometric studies and bladder strip tension/length studies were conducted on three groups (Sham, OVx, OVx + MI) 21 days after surgery. Means among groups were compared with ANOVA.

Results: KO studies: OVx was not associated with altered bladder compliance, voiding interval, NVC count, voiding threshold, maximum voiding pressure, per-void volume, or voiding AUC in WT mice. Compliance was greater in KO Sham than WT mice Sham $(p<0.05)$, with longer intervoid interval $(p<0.05)$ and a trend towards increased per-void volume compared to the other three groups. MI studies: OVx and OVx + MI mice gained weight over 21 days, Sham did not. No significant differences were found for basal and voiding threshold pressures, compliance, voiding interval, maximum voiding pressure, voided volume flow rates or voiding AUCs. In the strip studies, both raw and weight adjusted zero-tension length, maximum tension, passive tension at maximum active tension, and increase in length at maximum active tension did not differ among groups. 
Conclusions: Absence of the MIF gene is associated with altered bladder filling and diminished volume sensitivity, however oophorectomy eliminated this difference. Oophorectomy in intact animals was not associated with changes in bladder filling. Oophorectomy and MIF were not associated with changes in voiding function or detrusor tension generation characteristics. We conclude that MIF has a role in bladder filling control that negates any effect of oophorectomy.

Funding: NIH 5R01AG028657-02 (Kuchel PI)

Clinical Relevance: LUTS/Voiding Dysfunction

\section{Poster \# BS11}

\section{MOLECULAR CHANGES IN THE VAGINAL EXTRACELLULAR MATRIX OF LOXL1 KO MICE -AN ANIMAL MODEL OF PELVIC ORGAN PROLAPSE}

Bruna Couri, $\mathrm{MD}^{1}$, Ganesh Swaminathan B. Tech ${ }^{2}$, Mei Kuang, $\mathrm{PhD}^{3}$, Anand Ramamurthi, $\mathrm{PhD}^{2}$ and Margot Damaser, $\mathrm{PhD}^{4}$

${ }^{1}$ Depts. of Obstetrics \& Gyencology and Biomedical Engineering, Cleveland Clinic, Cleveland, $\mathrm{OH}{ }^{2}$ Dept. of Biomedical Engineering, Cleveland Clinic, Cleveland, $\mathrm{OH}$ \& Dept. of Biology, University of Akron, Akron, $\mathrm{OH} ;{ }^{3}$ Dept. of Biomedical Engineering, Cleveland Clinic, Cleveland, $\mathrm{OH} ;{ }^{4}$ Depts. of Biomedical Engineering, and Obstetrics \& Gynecology, and Glickman Urological \& Kidney Institute, Cleveland Clinic, Cleveland, $\mathrm{OH} \&$ Louis Stokes Veterans Affairs Medical Center, Cleveland, $\mathrm{OH}$

(Presented by: Bruna Couri, MD)

Introduction and Objectives: A deficient remodeling of the extracellular matrix (ECM) during pregnancy and post-partum could be related to pelvic organ prolapse (POP). In lysyl oxidase like-1 (Loxl1) knockout (KO) mouse model of POP, the condition develops after vaginal delivery and has been shown to correlates with disorganized elastin clusters. To establish a pathophysiologic basis for POP delvelopment, we sought to compare changes to the vaginal ECM homeostasis between nulliparous (N), multiparous non-prolapsed (MNP) and multiparous prolapsed (MP) Loxl1 KO mice, and nulliparous wild type (WT).

Methods: Primary cells were isolated from vaginal explants from age-matched N ( $n=3) ; \operatorname{MNP}(n=3) ; M P(n=3)$ and WT $(n=2)$ animals. Passage 2 cultured cells from each group, with 6 replicates each, were cultured for 21 days in DMEM/F12 medium with $10 \%$ fetal bovine serum, and then assayed for increase in DNA content and total elastic matrix level using Fastin assay. mRNA was isolated from cell cultures, then RTPCR was performed to assess the expression of tropoelastin (Eln), collagen 1a (Colla) and metalloproteinase (MMP) 2 and MMP 9.

Results: The ratio of total elastin to DNA (ng/ng) was significantly different and increased in the MP group when compared to all other groups, in the $\mathrm{N}$ group it was significantly reduced when compared to MNP and WT mice. RT-PCR showed that the Colla expression was significantly increased in MP mice compared to MNP mice. MMP9 expression was significantly increased in the $\mathrm{N}$ group when compared to the WT animals.

Conclusions: MP mice developed POP despite relatively higher collagen gene expression, suggesting impaired ECM structure. The results of this ongoing work suggest that pathophysiologic changes in Loxl1 KO mice, including elastin and collagen disorganization, may lead to, or be triggered by, prolapse, inducing a compensatory regenerative response, which is aberrant and intrinsically limited its ability to reinstate healthy ECM and thus prevent or reverse POP.

Funding: NIH RO1 HD059859 and the Cleveland Clinic Clinical Relevance: Pelvic Organ Prolapse

\section{Poster \# BS12}

\section{ACUTE URINARY RETENTION AND SOMATIC AND AUTONOMIC PELVIC NERVES INJURY INDUCED BY SIMULATED CHILDBIRTH TRAUMA IN RATS}

José Palacios ${ }^{1}$, Nicté Xelhuantzi, $\mathrm{PhD}^{1}$, Margot Damaser, $\mathrm{PhD}^{2}$ and Yolanda Cruz, $\mathrm{PhD}^{1}$

${ }^{1}$ Universidad Autonoma de Tlaxcala; ${ }^{2}$ Cleveland Clinic

(Presented by: José Palacios)

Introduction and Objectives: Postpartum urinary retention has been observed after long labor duration. The rat model of vaginal distention (VD) induces bladder hypoxia and urethra and pudendal nerve injury, which is associated with the development of stress urinary incontinence (SUI). The present study investigates whether vaginal distention using a balloon constricts the urethra inducing acute urinary retention, as well as damage to pelvic somatic and autonomic nerves.

Methods: Twenty Wistar virgin female rats were divided into 2 groups; vaginal distention (VD, $\mathrm{n}=10$ ), and sham ( $\mathrm{SH}, \mathrm{n}=10$ ). Anesthetized rats underwent 4 hours of VD (VD group), or the Foley catheter was introduced in the vagina but not distended (SH group). Immediately after the four hours, a laparotomy was performed and the pelvic bone removed to observe the lower urinary tract features and measure the length of relevant nerves ( $\mathrm{n}=8$ per group). In 6 rats, ( $3 \mathrm{VD}$ and $3 \mathrm{SH}$ ) the activity of the dorsal nerve of the clitoris (DNC) and cavernous nerve (CN) were recorded, and the pelvic nerve was electrically $(10 \mathrm{~Hz}, 4 \mathrm{~V}$, $0.4 \mathrm{~ms}$ duration) stimulated to induce bladder contraction. In the other four rats (2 VD, $2 \mathrm{SH}$ ) the bladder was collected for histology. A student's t-test was used to analyze the results with $\mathrm{P}<0.05$ indicating a significant difference between groups.

Results: In addition to stretching the motor branch of the sacral plexus, also known as the motor branch of the pudendal nerve, VD significantly increased the length of DNC, CN, and nerves of the bladder $(p<0.01)$. The balloon compressed the urethra to the pelvic bone avoiding urine expulsion, which significantly increased bladder length and urine volume $(p<0.01)$. Hematomas were observed, mainly in the bladder dome and neck. CN and DCN activity in response to perigenital skin stimulation was depleted in VD rats. Electrical stimulation threshold to induce bladder contraction increased significantly in VD rats $(p<0.01)$. Bladder histology demonstrated blood and leukocyte extravasation.

Conclusion: The results suggest that simulated childbirth trauma in rat damages somatic and autonomic nerves, and induces bladder overdistention as a collateral effect of bladder outlet obstruction during VD. Increased pelvic nerve threshold to induce bladder contraction may be result of damage to efferent nerves or detrusor impairment. We conclude that VD may be also used as a model for acute urinary retention.

Funding: CONACyT: YCG 183446, JLPG 4882

Clinical relevance: Female Urology-including Incontinence

\section{Poster \# BS13}

VALIDATION OF EXTERNAL URETHRAL SPHINCTER (EUS) ELECTROMYOGRAPHY (EMG) IN MICE 
Oi-Xiang Song, $M D^{1,2,3}$, Dan Li Lin, $M^{1,3}$, Brett Hanzlicek, $\mathrm{MS}^{3}$, Kangli Deng, $\mathrm{MD}^{1,3}$, Yinghao Sun, $\mathrm{MD}, \mathrm{PhD}^{2}$ and Margot Damaser, $\mathrm{PhD}^{1,3}$

${ }^{1}$ Department of Biomedical Engineering, the Cleveland Clinic, Cleveland, $\mathrm{OH} ;{ }^{2}$ Department of Urology, Changhai Hospital, the Second Military Medical University, Shanghai, PR, China; ${ }^{3}$ Louis Stokes Cleveland Veterans Affairs Medical Center, Cleveland, $\mathrm{OH}$ (Presented by: Qi-Xiang Song, MD)

Introduction and Objectives: Mice have been utilized for investigations into urinary incontinence and voiding dysfunction primarily because of their potential for investigating genetic aspects of these disorders. However, EUS EMG recordings in mice are not common, due to size constraints in these small animals. The goal of this study was to validate EUS EMG recordings simultaneous with cystometry and leak point pressure (LPP) testing on mice.

Methods: Eight female age-matched C57BL/6J mice were anesthetized and the pudendal canal was located bilaterally, followed by implantation of a polyethylene (PE-10) catheter into the bladder for cystometry and exposure of the urethra for simultaneous EUS EMG recording using a parallel bipolar electrode. LPP was measured at half capacity and was calculated by subtracting baseline pressure from peak pressure during testing. After acute bilateral pudendal nerve transection (PNT), LPP and EUS EMG recordings were repeated on the same mouse. EMG bursting activity during voiding was quantitatively analyzed in six of eight mice and presented as mean \pm standard error of the mean. Statistical comparisons of quantitative data before and after PNT and between baseline and peak LPP were made using a paired t-test with $\mathrm{p}<0.05$ indicating a statistically significant difference between groups.

Results: With the pudendal nerve intact, EUS EMG amplitude and firing rate increased significantly in response to LPP testing (from $38.9 \pm 8.4 \mu \mathrm{V}$ to $111.7 \pm 19.4 \mu \mathrm{V}$ and from $37.6 \pm 9.5 \mathrm{~Hz}$ to $142.6 \pm 15.0 \mathrm{~Hz}$, respectively). EUS EMG bursting activity during voiding was characterized with interbursting interval: $222.0 \pm 44.2 \mathrm{~ms}$; bursting duration: $85.6 \pm 6.2 \mathrm{~ms}$; intrabursting amplitude: $144.1 \pm 51.7 \mu \mathrm{V}$ and intrabursting firing rate: $135.4 \pm 29.3 \mathrm{~Hz}$. Bilateral PNT significantly decreased LPP (from $15.4 \pm 1.6 \mathrm{cmH} 2 \mathrm{O}$ to $7.3 \pm 0.8 \mathrm{cmH} 2 \mathrm{O}$ ) and eliminated EMG activity during cystometry, voiding, and LPP testing, validating that the EMG originated from EUS which is innervated by the pudendal nerve.

Conclusions: Compared to the results from our previous rat studies, LPP was reduced while EUS EMG amplitude and interbursting interval were increased in this mouse study. Our outcomes validate EUS EMG recordings in mice and suggest its utility as a measure of EUS function in investigations of urinary incontinence and voiding dysfunction in mice.

Funding: Dept. of Veterans Affairs

Clinical Relevance: Female Urology-including Incontinence

\section{Poster \# BS14}

\section{THE EFFECTS OF C5 HEMICONTUSION INJURY ON BLADDER FUNCTION IN RATS}

Courtney Shepard, MD, Timothy Ness, MD, PhD, Candace Floyd, PhD, Cary DeWitte, BS

$U A B$, Birmingham, Alabama

(Presented by: Courtney Shepard, MD)

Introduction and Objectives: Spinal cord injury is a significant cause of morbidity. C5 injuries are the most common location and most injuries are partial. Therefore, it is widely felt that the
C5 contusion injury model is currently the most clinically relevant. To our knowledge no study on the effects of this injury on bladder function has been performed. The primary goal of this study is to determine the effects of a C5 hemicontusion injury on bladder function in the acute, subacute, and chronic stages in rats. Additionally, systemic oxytocin administration has been shown to stimulate bladder contraction in healthy rats. A secondary objective of the study is to determine the effects of oxytocin in injured rats.

Methods: After anesthesia was induced, male Sprague-Dawley rats underwent C5 laminectomy and controlled unilateral contusion injury using a standardized weight drop method. The control rats underwent similar cervical surgery without laminectomy. All rats received the same postoperative care. They were allowed to recover for either 3,8 or 15 days post injury. On the day of testing the rats were anesthetized with isoflurane and urethane. A central venous line was placed and the bladder was exposed and the dome cannulated using a catheter with an in-line pressure transducer. A continuous infusion of normal saline was then initiated and intravesical pressures continuously recorded. Voiding events were noted and amounts measured. Oxytocin $10 \mathrm{u} / \mathrm{kg}$ was administered through the central line and the effects of the medication were recorded. Cystometrographic findings were compared between groups using Student's t test.

Results: Bladder function was distinctly different at the various time intervals. All injured rats showed more frequent but ineffective contractions. At 3 days post injury, rats had higher baseline bladder pressures with weaker contractions. By 15 days, the baseline pressure had normalized but the rats demonstrated significantly higher voiding pressure. Administration of oxytocin caused higher baseline pressure and weaker contractions in all injured rats. At 3 days, rats had significantly more frequent and more effective bladder contractions. This was also noted at 8 and 15 days, but with diminishing effect over time.

Conclusions: C5 hemicontusion injury causes predictable bladder dysfunction which changes with time since injury. Oxytocin shows promise is pharmacologic treatment in the acute and possibly subacute injury settings.

Clinical Relevance: Neurogenic Bladder

\section{Poster \# BS15}

SAFETY AND EFFICACY OF THE UROGUIDE, A NOVEL STRESS URINARY INCONTINENCE DEVICE IN THE FEMALE RODENT

Shida Li, BS ${ }^{1}$, Franklin Yao, BS ${ }^{1}$, Melissa Laudano, MD $^{2}$, Daniel Lee, $\mathbf{M D}^{2}$, Fujun Zhao, $\mathbf{M D}^{2}$, Bilal Chughtai, $\mathbf{M D}^{2}$ and Richard Lee, $\mathrm{MD}^{2}$

${ }^{1}$ Urova Medical Inc. Memphis, TN; ${ }^{2}$ Department of Urology, Weill Medical College of Cornell University, New York, NY (Presented by: Shida Li, BS)

Introduction and Objectives: Stress urinary incontinence (SUI) is a prevalent condition affecting women's health. The Uroguide is a novel suburethral implant that acts to redirect and disperse the biomechanical forces acting upon the urethra. The purpose of this study was to determine the effects of the Uroguide in restoring leak point pressure (LPP) in a rodent model for SUI.

Methods: A total of 36 Sprague-Dawley retired female breeder rodents was randomly assigned to 4 groups: 1$)$ control $(n=5), 2)$ SUI only $(n=5), 3)$ SUI + sham implant placement $(n=5)$, and 4) SUI + Uroguide $(n=20)$. SUI was induced through pudendal nerve transection after baseline LPP measurements. Cystom- 
etry was performed via suprapubic catheter implanted into the bladder of the rodents. LPP measurements were taken at baseline, 1 and 5 weeks. Uroguide implants were placed after week 1 LPP measurement. Statistical analysis was performed using the Kruskal-Wallis and Friedman tests. The study was funded by Urova Medical, Inc.

Results: A total of 28 rodents survived the end of the study: control $(n=5)$, SUI $(n=2)$, SUI + sham implant placement $(n=4)$, and SUI + Uroguide $(n=17)$. Declines in the LPP were seen for all SUI animals at week 1 (Table $1, p=0.02$ ). Uroguide implants were placed successfully with no evidence of infection or erosion. LPP was successfully restored in the Uroguide animal group ( $p=0.006)$ by week 5 .

Conclusions: Implantation of the Uroguide appears to restore LPP in female SUI rodents. Future research is needed to verify its efficacy in larger animal groups and to further evaluate its safety and biocompatibility.

\begin{tabular}{|c|c|c|c|c|c|}
\hline & Control & $\begin{array}{l}\text { SUI } \\
\text { only }\end{array}$ & $\begin{array}{l}\text { SUl+gham } \\
\text { implant }\end{array}$ & $\begin{array}{c}\text { SUIt } \\
\text { Uroguide }\end{array}$ & p-value \\
\hline Median LPP, $\pm \mid O R$ & $54.0 \pm 20.6$ & $44.2 \pm 0$ & $50.6 \pm 18.8$ & $46.0 \pm 14.4$ & 0.32 \\
\hline Median LPP & $38.4 \pm 14.0$ & $22.7 \pm 0$ & $22.1 \pm 12.1$ & $22.2 \pm 4.8$ & 0.02 \\
\hline Median LPP, smat $t \mid O R$ & $48.0 \pm 12.7$ & $31,1+0$ & $18.5 \pm 17.8$ & $45.8 \pm 10.9$ & 0.006 \\
\hline
\end{tabular}

\section{Poster \# BS16}

\section{CALF URODYNAMICS: A NOVEL LARGE-ANIMAL UROLOGIC} MODEL

Elizabeth Ferry, $\mathrm{MD}^{1,2}$, Steve Majerus, $\mathrm{MS}^{1,3}$, Brian Balog, $\mathrm{BS}^{1,4}$, Matthew Streicher, $\mathrm{MS}^{1,4}$, Hui Zhu, MD, $\mathrm{PhD}^{1,2}$ and Margot Damaser, $\mathrm{PhD}^{1,4,5}$

${ }^{1}$ Louis Stokes Cleveland Veterans Affairs Medical Center, Cleveland, $\mathrm{OH} ;{ }^{2}$ Urology Institute, University Hospitals Case Medical Center, Cleveland, $\mathrm{OH} ;{ }^{3}$ Department of Electrical Engineering and Computer Science, Case Western Reserve University, Cleveland, $\mathrm{OH} ;{ }^{4}$ Department of Biomedical Engineering, Cleveland Clinic, Cleveland, $\mathrm{OH} ;{ }^{5}$ Glickman Urological and Kidney Institute, the Cleveland Clinic, Cleveland, $\mathrm{OH}$

(Presented by: Elizabeth Ferry, MD)

Introduction and Objectives: While swine have long been the standard large-animal model for urologic endoscopy, they are not an ideal choice for assessing effects of ambulation. Calves are more docile and may be halter-trained, for clinically applicable long-term survival data acquisition. We present a novel large-animal model for cystoscopic procedures and urodynamics (UDS).

Methods: A nonsurvival $59.2 \mathrm{~kg}$ female Jersey calf was anesthetized using isoflurane following an IACUC-approved protocol. A $26 \mathrm{~F}$ nephroscope was introduced atraumatically under direct vision into the vestibule, then into the urethra, ventral to the vagina. The large urethral diverticulum was noted and traversed. The urethra then made a 45 degree turn ventrally, ending at the bladder neck. Retrograde pyelograms and cystogram were obtained to delineate anatomy. A glidewire was placed via the nephroscope for 16F Foley catheter placement. The urethral length was measured on retraction. The UDS pressure transducer was zeroed and the fill rate verified at $40 \mathrm{ml} / \mathrm{min}$. The Foley catheter was connected to the UDS tubing and non-differential filling UDS was performed. A custom receiver demodulated the data and reconstructed it as an analog waveform for collection with a data acquisition system at $1000 \mathrm{~Hz}$. Necropsy was performed.

Results: The urethral diverticulum had a $150 \mathrm{~mL}$ capacity. The urethral length from meatus to diverticulum was $2 \mathrm{~cm}$, and $7 \mathrm{~cm}$ from meatus to bladder neck. Progressive urethral dilation was not required. Cystogram revealed a large, smooth walledbladder with one small diverticulum at the dome. No reflux was observed. Retrograde pyelograms demonstrated unobstructed single systems bilaterally. Resting bladder pressure at volumes $<800 \mathrm{~mL}$ were $16-18 \mathrm{~cm}$ H2O. Total anesthetized bladder capacity was approximately $1200 \mathrm{~mL}$ with pressures up to $90 \mathrm{~cm} \mathrm{H2O}$, though minor overflow incontinence was noted starting $850 \mathrm{~mL}$. Appropriate pressure changes with manual compressions of the bladder were noted. Normal spontaneous voiding was not observed. Minimal filling $(3 \mathrm{~mL})$ of the Foley balloon obstructed the urethra. On necropsy, the bladder weighed 59.0g.

Conclusions: This novel model for large-animal urodynamics offers advantageous bladder capacity, compliance, and resting pressure. Further studies with alternative anesthesia regimen are required to ascertain the ability of the anesthetized calf to void spontaneously.

Funding: Dept. of Veterans Affairs RR\&D Merit Review 1I01RX000443-01

Clinical Relevance: Urodynamics

\section{Poster \# BS17}

\section{COMPUTER SIMULATION OF PELVIC FLOOR STRESS DISTRIBUTION AND ITS IMPLICATIONS IN URINARY INCONTINENCE}

Chi-Lun Lin, PhD, candidate ${ }^{1}$, Guangjian Wang, $\mathrm{PhD}^{2}$, Arthur Erdman, PhD $^{1}$ and Gerald Timm ${ }^{2}$

${ }^{1}$ Dept. of Mechanical Engineering, University of Minnesota, Minneapolis, $M N{ }^{2}$ Dept. of Urology, University of Minnesota, Minneapolis, $M N$

(Presented by: Gerald Timm)

Introduction and Objectives: Mechanical stress applied to pelvic continence mechanisms is a major risk factor for urinary incontinence (UI). Thus, even in healthy young female athletes excessive vigorous jumping can cause urine leakage. A stressful event could temporarily or eventually permanently compress and injure continence-related pelvic nerves and muscular structures resulting in UI. Our objectives are to identify the stress distribution in a human pelvic computer model using the finite element analysis (FEA), and to investigate the effect of pressure on nerve physiology.

Methods: A female pelvic FEA model is constructed with MRI scans from a volunteer and tissue mechanical properties from the literature. The stress distribution in pelvic structures in response to a jump is simulated using ABAOUS/Explicit. Nerve compound action potentials (CAP) are recorded in freshly isolated swine phrenic nerves. Standard electrophysiological methods are used. A nerve recording chamber is custom modified to study the effect of pressure on CAP.

Results: A jumping load as a subject jumps from 1-foot height to the ground exerted a force on and caused a distinct distribution of stress in pelvic floor muscles. The highly stressed areas in pelvic floor muscles include illiococcygeus adjacent to lateral pelvic walls, puborectalis, and bladder neck-urethra. Four nerve samples have been tested. One hundred mmHg for 10 minutes totally blocked CAP in one sample, reduced CAP conduction velocity $(\sim 74 \%)$ and increased CAP duration $(\sim 110 \%)$ in another two samples. No effect was observed in the fourth sample. Changes in CAP returned to baseline levels after ten minutes recovery.

Conclusions: Distinct stress distribution in pelvic floor muscles and significant block of nerve compound action potential by compression is observed by using FEA simulation and electro- 
physiological methods, respectively. It remains to be determined if these highly stressed areas are significantly associated with continence-related nerves. If irreversible nerve injury can result in permanent incontinence, strategies to prevent such an injury may prove to be useful in the treatment of urinary incontinence.

Clinical Relevance: Female Urology-including incontinence

\section{Poster \# BS18}

\section{CYSTOSCOPIC SUBMUCOSAL BLADDER DEVICE} IMPLANTATION: A FEASIBILITY STUDY

Elizabeth Ferry, $M^{1,2}$, Steve Majerus, $M^{1,3}$, Hui Zhu, MD, PhD ${ }^{1,2}$, Steven Gaverick, $\mathrm{PhD}^{1,3}$ and Margot Damaser, $\mathrm{PhD}^{1,4,5}$

${ }^{1}$ Louis Stokes Cleveland Veterans Affairs Medical Center, Cleveland, $\mathrm{OH} ;{ }^{2}$ Urology Institute, University Hospitals Case Medical Center, Cleveland, $\mathrm{OH} ;{ }^{3}$ Department of Electrical Engineering and Computer Science, Case Western Reserve University, Cleveland, $\mathrm{OH} ;{ }^{4}$ Department of Biomedical Engineering, Cleveland Clinic, Cleveland, $\mathrm{OH} ;{ }^{5}$ Glickman Urological and Kidney Institute, the Cleveland Clinic, Cleveland, $\mathrm{OH}$

(Presented by: Elizabeth Ferry, MD)

Introduction and Objectives: Current methods of determining bladder pressure are limited in their snap-shot nature and artificial environment, in addition to not being amenable to chronic or ambulatory settings. Chronic bladder pressure monitoring could enable conditional neuromodulation, increasing effectiveness and efficiency. An ideal chronic pressure sensor must be wireless, fully internalized, nonirritative, out of contact with the urine, and placed in a minimally-invasive manner. This study aims to evaluate the safety and feasibility of cystoscopic implantation of inert devices into the suburothelium in a large animal model.

Methods: A nonsurvival $59.2 \mathrm{~kg}$ female Jersey calf was anesthetized following an IACUC-approved protocol. Cystourethroscopy was performed using a 26F nephroscope for Foley catheter placement over a wire. Cystogram was performed. The nephroscope was replaced and a suburothelial pocket was made using an electrode and cystoscopic scissors. The device was placed into the suburothelial pocket by placing the sheath in the pocket mouth, removing the lens, and advancing the device through the sheath. Placement adjustments were performed using the rigid graspers. This process was repeated to test insertion feasibility in several pockets. Fluoroscopic CTrendered cystograms were obtained to assess bladder integrity and device location. Necropsy was subsequently performed.

Results: Pocket placement was subjectively more straightforward immediately cephalad to, but not involving, the trigone. Cystograms before and after initial pocket creation were identical and confirmed bladder integrity. Visually, the device was placed completely within the suburothelial pocket in two peri-trigone areas, without urothelium interposition. Adequate placement closer to the dome was subjectively more difficult. Final cystogram was concerning for a small contained perforation near the dome. Necropsy did not reveal deep perforation grossly, or on filling with Toluidine blue. Pocket locations were confirmed, as well as gross appropriate depth.

Conclusions: Small devices may be successfully placed in a suburothelial position using minimally invasive, standard techniques. Posterior wall locations immediately cephalad to the trigone appear to be optimal. Further survival studies are needed to determine long-term outcomes.
Funding: Dept. of Veterans Affairs RR\&D Merit Review 1I01RX000443-01

Clinical Relevance: Neuromodulation

\section{Poster \# BS19}

\section{TRANSCRIPTIONAL REGULATION OF CRF GENE EXPRESSION}

Lizath Aguiniga, Anthony Schaeffer, MD, David Klumpp, PhD Northwestern, Chicago, IL

(Presented by: Lizath Aguiniga)

Introduction and Objectives: Corticotropin-releasing factor (CRF) has been well established as a key mediator of voiding control and stress responses, where increased CRF levels in Barrington's nucleus induce urinary retention and bladder dysfunction. Arachidonic acid (AA) metabolites have been shown to modulate CRF expression, however the transcriptional mediators of this modulation are unknown.

Methods: We used MIRAGE software to identify candidate transcription factor binding sites in a $1 \mathrm{~kb}$ region of the human CRF gene promoter. We identified a peroxisome proliferatoractivated receptor (PPAR) and two Xenobiotic Responsive Element (XRE) sites as candidate mediators of AA-dependent CRF induction. Site-directed mutations of the PPAR and XRE sites were generated in a CRF-luciferase reporter plasmid. We evaluated expression of WT and the mutants in 293T cells for their responses to AA and CAMP.

Results: Mutation of XRE1 resulted in significantly decreased basal CRF expression, while mutation of XRE2 or PPAR had modest effects. However upon AA induction, the PPAR mutant had increased CRF expression compared to WT, whereas XRE1 had decreased expression. The double mutation of XRE1 and XRE2 resulted in decreased responsiveness to CAMP.

Conclusions: These results suggest CRF gene expression is modulated by factors that bind to the PPAR and XRE sites. Continued studies will examine the role of such factors in modulating voiding during stress.

Funding: Research reported in this publication was supported by the National Institute of Allergy And Infectious Diseases of the National Institutes of Health under Award Number F31AI106357.

Clinical Relevance: IC and Pelvic Pain - UTI/Inflammatory

\section{Poster \# BS20}

\section{URINARY NERVE GROWTH FACTOR LEVELS ARE INCREASED IN FEMALES WITH ANATOMIC BLADDER OUTLET OBSTRUCTION}

Julie Stewart, $\mathrm{MD}^{1}$, Rose Khavari, $\mathrm{MD}^{1}$, Robert Chan, $\mathrm{MD}^{1}$, Alvaro Munoz, $\mathrm{PhD}^{1}$, Timothy Boone, $\mathrm{MD}^{1}$

and Sophie Fletcher, $\mathbf{M D}^{2}$

${ }^{1}$ Department of Urology, Houston Methodist Hospital, Houston, TX; ${ }^{2}$ Kaiser Permanente, Santa Rosa, CA

(Presented by: Robert Chan, MD)

Introduction and Objectives: Bladder outlet obstruction (BOO) in women can be a diagnostic challenge. The etiologies of BOO in female patients vary widely with no clear consensus on its definition. The urinary biomarker, nerve growth factor (NGF), has been previously shown to be elevated in male patients with $\mathrm{BOO}$ and other lower urinary tract symptoms (LUTS). No published studies have examined NGF as a potential urinary biomarker in females with BOO. The aim of this prospective, IRB approved, pilot study is to evaluate NGF levels in women with 
anatomic BOO resulting from pelvic organ prolapse (POP) and/ or previous incontinence surgery.

Methods: From January -September 2012, all female patients referred for evaluation and management of $\mathrm{BOO}$ from POP or previous incontinence surgeries were screened for enrollment. Inclusion criteria included: elevated post-void residual (PVR), valsalva voiding on urodynamics (UD) or urinary peak flow (Omax) $\leq 12 \mathrm{~mL} / \mathrm{s}$. A control group of 10 asymptomatic agematched female volunteers was also recruited. In all subjects, urinary NGF and creatinine $(\mathrm{Cr})$ levels were measured by enzyme-linked immunosorbent assay. The total urinary NGF levels were normalized to the urinary $\mathrm{Cr}$ concentrations (NGF/ $\mathrm{Cr}$ ).

Results: A total of 7 female patients with anatomic BOO (mean age of $64 \pm 13$ years) and 10 female control subjects (mean age of $62 \pm 7$ years) were recruited. Etiologies of BOO in the study group included POP $(n=1)$, previous incontinence surgery $(n=4)$, and a combination of POP and previous incontinence surgery $(n=2) .6 / 7$ of the patients underwent UD, all with evidence of valsalva voiding. The mean Omax for these 6 patients was $5.3 \mathrm{~mL} / \mathrm{s}$ (SD 4.3). The one patient who did not undergo UD had frank urinary retention with a PVR of $1200 \mathrm{~mL}$. The urinary NGF/Cr levels in the study patients with BOO (mean \pm standard error $22.95 \pm 6.512 \mathrm{pg} / \mathrm{mg}$ ) were significantly higher $(p=0.0027)$ than the levels in the age-matched control group ( $5.595 \pm 0.656 \mathrm{pg} / \mathrm{mg})$. After surgical treatment with release of prior anti-incontinence procedure or POP repair, the urinary $\mathrm{NGF} / \mathrm{Cr}$ level significantly decreased to $7.48 \pm 0.962 \mathrm{pg} / \mathrm{mg}(\mathrm{p}=0.0001)$.

Conclusions: In this study, female patients with anatomic BOO resulting from POP and/or previous incontinence surgery had significantly higher urinary NGF/Cr levels when compared to age-matched controls. After surgical correction, the urinary $\mathrm{NGF} / \mathrm{Cr}$ levels were significantly reduced.

Clinical Relevance: Female Urology-including incontinence

\section{Poster \# BS21}

\section{DIABETIC BLADDER DYSFUNCTION AND DETRUSOR} PDGFRALPHA + CELLS

Byoung Koh, Haeyeong Lee, PhD, Lauren Peri, BS, Kenton Sanders, PhD, Sang Don Koh, MD, PhD UNR

(Presented by: Byoung Koh)

Introduction and Objectives: The most common and bothersome lower urinary tract complication of DM is diabetic bladder dysfunction (DBD). Clinical manifestations of DBD involve a combination of storage and voiding bladder problems. Although a number of clinical studies have reported detrusor overactivity as the most frequent finding, ranging from $39 \%$ to $61 \%$ of diabetic patients, the pathophysiological mechanisms remain unclear. Streptozotocin (STZ) induced Type 1 Diabetes Mellitus Animal Models is a well-accepted technique to induce type $1 \mathrm{DM}$. This animal exhibited increased voiding frequency, residual volume, and contraction-duration. However, the exact pathophysiological mechanism of increased detrusor responsiveness in early stage DM is unclear. There is an abundance of PDGFR $\alpha+$ cells in detrusor muscles. This cell involves the membrane stabilization during filling. Thus we investigate the molecular and protein phenotype of this unique population of cells in STZ-induced type 1 DM mouse to characterize the role of these cells that contributes to development of DBD.
Methods: We applied molecular approaches, immunohistochemistry and tension measurement. We analyzed changes in the molecular expression of Pdgfrÿ!, Kcnn and P2ry transcripts and also characterize the PDGFR $\alpha$ immunoreactivity in control and STZ-induced type 1 DM detrusors. Since SK channels are highly expressed in detrusor PDGFR $\alpha+$ cells, we tested the effect of SK channel blockers on transmural nerve-evoked (EFS) contractions in both mice.

Results: In quantitative analysis of transcripts, Pdgfr $\alpha$, Kcnn3 and P2ry1 transcripts in STZ-induced type 1 DM detrusor were significantly decreased compared with control detrusor. Immunohistochemistry revealed the reduced immunoreactivity of PDGFR $\alpha$ in STZ-induced type 1 DM detrusors. In isometric force measurements, control mouse showed frequency $(1-20 \mathrm{~Hz})$ dependent contractions and apamin treatment increased contractile force in responses to EFS. However, STZinduced type 1 DM detrusor does not demonstrate apaminsensitivity in EFS-evoked contractions. These data suggest that down-regulation of PDGFR $\alpha$ in STZ-induced type 1 DM might involve the development of bladder overactivity during early stage of DBD.

Conclusions: The present data support the hypothesis that disturbance of PDGFRÿ! receptors and SK channels in PDGFR $\alpha+$ cells during $\mathrm{DM}$ can be a main factor to induce detrusor overactivity.

Funding: Supported DiaComp Pilot \& Feasibility project, 13GHSU267)

\section{Poster \# BS22}

\section{ANALYSIS OF ERODED AND NON-ERODED TRANSVAGINAL MESHES: IS THERE A DIFFERENCE IN THE MICROBIOLOGY?}

Ketul Shah, MD, Andrew Windsperger, MD, Brian Flynn, MD

Denver, $C O$

(Presented by: Ketul Shah, MD)

Introduction and Objectives: Subclinical contamination of polypropylene mesh has been demonstrated by bacteriological studies during mesh implantation and explantation. When vaginal mesh erosion is detected, it raises the question of mesh colonization as a risk factor for erosion or whether erosion exposes the mesh to vaginal bacteria. The aim of this study is to perform bacteriological analysis of eroded and non-eroded transvaginal meshes to detect if there is a difference in microbiology.

Methods: We retrospectively reviewed medical records of 50 patients who presented with mesh related complications requiring transvaginal removal of polypropylene mesh from vagina or lower urinary tract. The excised mesh was placed in a sterile container and underwent aerobic, anaerobic and fungal analysis in the microbiology department.

Results: 50 patients underwent surgical removal of mesh through transvaginal or combined transvaginal/abdominal approaches from October 2011 to November 2012. The age ranged from 26 to 79 years (mean 52). Time since initial procedure to mesh explantation was 0.4 to 12 years (mean 3.1). Mesh placement was performed for stress urinary incontinence $(n=29)$, pelvic organ prolapse $(n=6)$ and combined $(n=15)$ cases. Indications for mesh removal included painful mesh $(n=27)$, vaginal erosion $(n=10)$, lower urinary tract erosion $(n=9)$ and recurrent SUI $(n=4)$. Pelvic pain $(n=32)$ was the most common presenting symptom followed by dyspareunia $(n=28)$ and recurrent SUI $(n=13)$. Positive mesh culture was found in $40(80 \%)$ patients and no bacterial growth was seen in $10(20 \%)$ patients. Twenty two patients had more than one 
organism in culture. A total of 23 pathogenic and 26 nonpathogenic organisms were identified. Enterococcus $(n=6)$ was the most common pathogenic organism and coagulase negative staphylococcus $(n=8)$ was the most common nonpathogenic organism. Pathogenic organisms were found in patients with painful mesh (52\%), erosion into urinary tract (83\%) and vaginal erosion (20\%) (Fig. 1)

Conclusion: Colonization of vaginally implanted mesh occurs frequently and bacterial infection may account for pelvic pain in patients with painful mesh and dyspareunia.

Funding: None

Clinical Relevance: Reconstruction

\section{Poster \# BS24}

\section{EFFECT OF BEHAVIORAL THERAPY FOR URGE INCONTINENCE ON BRAIN FUNCTION}

Neil Resnick, MD, Stasa Tadic, MD, Becky Clarkson, PhD, Andrew Murrin and Derek Griffiths, PhD

University of Pittsburgh, Pittsburgh, PA

(Presented by: Becky Clarkson, PhD)

Introduction: Urge urinary incontinence is common and troublesome in older people, and the mechanism of disease is poorly understood. The study aim was to use biofeedback assisted pelvic floor exercises (BFB) as a probe to explore the cerebral mechanisms involved in UUI and changes associated with symptom improvement. We identified a priori the dorsal anterior cingulate cortex and supplementary motor area (dACC/SMA), the medial pre-frontal cortex (mPFC) and the right insula as regions of interest in the continence mechanism and assessed change in activity before and after BFB.

Methods: Sixty community-dwelling women over 60 years of age with UUI and $>5$ leakage episodes per week were recruited to have 4 BFB sessions to improve their UUI symptoms. Baseline and post therapy assessments included: physical exam; 3-day voiding and 1-day pad diary; urodynamics; and functional MRI during a standardized bladder filling protocol. Eleven continent controls had the same baseline assessment. Responders to treatment had $>50 \%$ reduction in \# of leakage episodes.

Results: Subjects: mean age 72.3y (responders: 70.1; nonresponders $73.8 ; p=0.14$ ). Controls: mean age $65 y$. Treatment: With BFB, median frequency of UUI episodes decreased from 3.3 to $1.7 / 24 h(\mathrm{P}<0.0001)$. 24/60 (40\%) were responders. Median UUI frequency decreased from 3.0 to 0.3 episodes/ $24 \mathrm{~h}$ in responders and 3.4 to $2.7 / 24 \mathrm{~h}$ in non-responders. 9/60 subjects $(15 \%)$ became dry. Baseline UUI frequency did not differ between responders and non-responders $(P=0.5)$. fMRI: $\mathrm{dACC} / \mathrm{SMA}$ was strongly activated at baseline in responders, tending to decrease after BFB in reaction to successful treatment, as expected. mPFC was strongly deactivated at baseline (only non-responders); it did not change significantly after treatment. $\mathrm{mPFC}$ deactivation predicted but did not mediate failure of UUI to improve. Insula was activated in responders at baseline and unchanged after BFB. Activation was much less pronounced in non-responders. Insula activation tended to predict success of BFB but not mediate it. fMRI results in responders did not differ significantly from controls $(P>0.7$ in every case). The mPFC deactivation seen in non-responders was absent in controls.

Conclusion: Women showing symptom improvement after biofeedback also show change in brain function as a consequence of treatment. Those refractory to treatment show a different initial pattern of activity suggesting that they represent a different phenotype.

clinical relevance: Female Urology-including Incontinence

\section{Poster \# BS25}

\section{FUNCTIONAL-MRI DURING BLADDER CYSTOMETRY IN SPINAL CORD-INJURED AND INTACT FEMALE RATS}

Kelvin Wong, $\mathrm{PhD}^{1}$, Timothy Boone, $\mathrm{MD}, \mathrm{PhD}^{2}$ and Alvaro Munoz, $\mathrm{PhD}^{1}$

${ }^{1}$ Houston Methodist Research Institute, Houston, TX; ${ }^{2}$ Houston Methodist Hospital, MHRI and VA-Medical Center, Houston, TX (Presented by: Alvaro Munoz, PhD)

Introduction and Objectives: The afferent arm of the micturition reflex is initiated by sensory signals in the bladder and transmitted to brain centers for storage \& release of urine. Our objective was to determine brain activation using functionalmagnetic resonance imaging (fMRI) in intact and spinal cord injured (SCI) rats during isovolumetric bladder contractions (IBC).

Methods: Female Sprague-Dawley rats with SCI (complete T8/ T9 transection) were evaluated after 28 days. Urethaneanesthetized rats had isovolumetric cystometry (CMG) during fMRI via a suprapubic catheter \& closed urethra. Saline infusion stopped at $100 \mathrm{cmH} 2 \mathrm{O}(\mathrm{cmw})$. CMG data were analyzed with ttest, expressed as mean $+/-S E M$, and $p<0.05$ statistically significant. Simultaneous fMRI was conducted with a 9.4T Bruker MRI system using a 4-elements receiver array and a quadrature volume transmit coil. 2D gradient echo-planar imaging was used to evaluate blood oxygenation level dependent (BOLD) responses in the brain during IBC and compared to the empty bladder. Group analysis was conducted based on mixed effect analysis with FMRIB Software Library v6.0 (http:// fsl.fmrib.ox.ac.uk/fsl/fslwiki/) and a cluster threshold of $\mathrm{Z}>2$. 5 with significance threshold of $p=0.05$.

Results: Isovolumetric pressure was $42+/-4 \mathrm{cmw}$ in intact $(\mathrm{n}=7)$ and $43+/-9 \mathrm{cmw}$ in SCI rats $(\mathrm{n}=4)$. IBC amplitude was $57+/-4$ in intact and $5+/-0.5 \mathrm{cmw}$ in SCI rats $(\mathrm{p}<0.001)$. Intercontractile interval was $99+/-16 \mathrm{~s}$ in control and $17+/-$ $3 \mathrm{~s}$ in SCI rats $(p<0.01)$. In intact rats $(n=6)$, mixed effect analysis of IBC showed clustered brain activation in hippocampus, ectorhinal cortex, dentate gyrus, thalamus nucleus, septal nucleus, primary/secondary motor cortex, primary somatosensory cortex as well as in the periaqueductal gray matter. SCI rats did not exceed the Z-threshold during IBC. Motion contaminated data were identify and excluded as pseudo brain activation due to correlated IBC motion.

Conclusion: We standardized a suitable protocol to study supraspinal activation during reflexive micturition using fMRI. Small contractions in SCI rats may represent neurogenic detrusor overactivity caused by increased excitability of afferent pathways without brain activation.

Funding: Methodist \& Brown Foundations

Clinical Relevance: Neurogenic Bladder
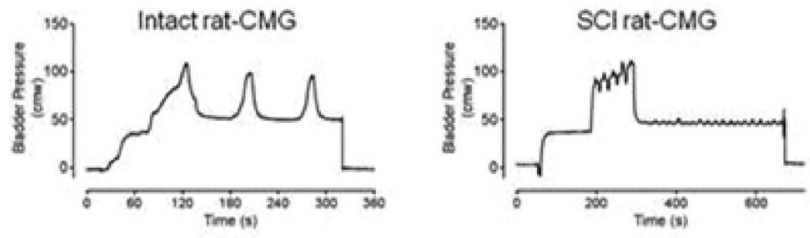

Intact rat BOLD fMRI (Contraction-Empty)

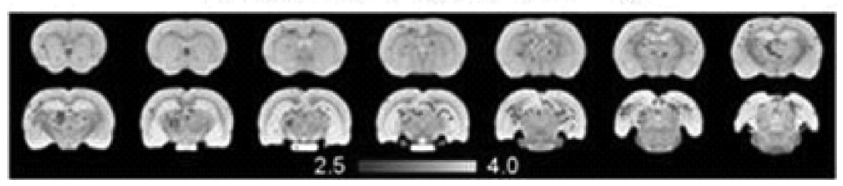




\section{Poster \# BS26}

MYOSIN VA REGULATES EXCITATORY NEUROTRANSMISSION IN THE BLADDER

Hongying Cao, $\mathrm{MD}^{1}$, Vivian Cristofaro, $\mathrm{PhD}^{2}$, Raj K. Goyal, $\mathrm{MD}^{1}$ and Maryrose P. Sullivan, $\mathrm{PhD}^{1}$

${ }^{1}$ Boston VA Healthcare System, Boston; ${ }^{2}$ Boston VA Healthcare System, Harvard Medical School

(Presented by: Vivian Cristofaro, PhD)

Introduction and Objectives: Excitatory neurotransmission in the bladder is predominantly comprised by an acetylcholine (Ach)-mediated cholinergic component and an ATP-mediated purinergic component. We have previously shown that deficiency of non-muscle myosin $\mathrm{Va}$, a motor protein involved in neurotransmitter exocytosis from secretory vesicles, was associated with a decrease in the purinergic but not the cholinergic component of bladder contractions. This study was aimed to further investigate the contribution of myosin Va to excitatory neurotransmission in the bladder.

Methods: The expression of myosin Va was investigated in bladder smooth muscle tissue from wild type C57BL/6J (WT) and myosin-Va deficient (DBA) mice by western blotting. The distribution of myosin-Va in detrusor tissue was evaluated by double label immunofluorescence confocal microscopy. Bladder tissue from WT and DBA mice were mounted in organ baths. Neurotransmitter release was induced by electrical field stimulation (EFS) and aliquots were collected after stimulation at increasing frequencies. ATP levels released from tissue by EFS were measured from aliquots by luciferin-luciferase assay and compared between WT and DBA samples.

Results: Myosin Va expression was detected in WT bladder protein lysates, while little expression was found in DBA bladder. Myosin Va was co-localized with the neuronal marker synaptophysin, indicating its neuronal distribution. In addition, positive immunoreactivity for Myosin Va co-localized with staining for SLC17a9, a vesicular transporter of ATP. The levels of ATP released upon EFS were significantly higher in WT bladders compared to DBA.

Conclusions: Myosin Va plays a role in purinergic neurotransmission by transporting vesicles containing ATP to varicosity membranes. These findings advance our understanding of the molecular basis of smooth muscle neurotransmission in the bladder, and may be relevant under pathologic conditions associated with alteration of the purinergic component of bladder contraction.

Funding: Supported by Medical Research Service, Department of Veterans Affairs, Washington, DC and NIDDK Grant DK06287.

\section{Poster \# BS27}

THE EFFECTS OF BILATERAL BIPOLAR SACRAL NEUROSTIMULATION ON URINARY BLADDER ACTIVITY DURING FILLING BEFORE AND AFTER IRRITATION IN A RAT MODEL

Alexis Dieter, $\mathrm{MD}^{1}$, Danielle Degoski, $\mathrm{BS}^{2}$, Paul Dolber, $\mathrm{PhD}^{1,2}$ and Matthew Fraser, $\mathrm{PhD}^{1,2}$

${ }^{1}$ Duke University Medical Center, Durham, NC; ${ }^{2}$ Durham Veterans Affairs Medical Center, Durham, NC

(Presented by: Alexis Dieter, MD)

Introduction and Objectives: Previous studies performed in our laboratory have shown that normal bladder filling and urine storage in rats is associated with a series of base-to- dome smooth muscle contractile waves that register cystometrically as low amplitude non-voiding contractions (NVC). We sought to determine if these NVCs were affected by sacral neurostimulation (SNS) and if we could simultaneously optimize the effect of SNS for future pharmacological studies of the mechanisms of action.

Methods: Bilateral bipolar SNS was performed in ten female urethane anesthetized Sprague-Dawley rats at the L6-S1 trunks with stimulus parameters of $10 \mathrm{~Hz}, 0.1 \mathrm{msec}$ pulse duration and $0.15-0.80 \mathrm{~mA}$ (below motor threshold). Transvesical cystometry was performed before and during SNS under conditions of control (saline, $\mathrm{N}=10$ ) and irritation [0.25\% acetic acid (AA), $\mathrm{N}=5$ ]. Wilcoxon ranked sum test was used to examine effect of SNS under saline infusate $(N=10)$. Nonparametric two-way analysis of variance for repeated measures (NP RM 2-ANOVA) was performed to analyze the effects of SNS under irritated conditions $(\mathrm{N}=5)$.

Results: Functional bladder capacity (FBC) and NVC count were significantly increased during SNS under both control and irritation ( $p<0.01$ for all). In 6 instances ( 4 in control, 2 in irritation), micturition reflexes were completely inhibited by SNS resulting in overflow incontinence. Filling compliance, NVC period and NVC maximum amplitude were not affected by SNS ( $p>0.05$ for all). NP RM 2-ANOVA revealed increased FBC and NVC count during SNS under both control and irritation ( $p=0.004$ for both, $N=5$ rats). Linear regression analysis of NVC count versus FBC revealed a slope significantly different than zero, independent of control or irritation (slope $49.06 \pm 14.33, p=0.0013$ )

Conclusions: This model achieved reliable, reversible and robust increases in FBC, up to overflow incontinence, at stimulation intensities below motor threshold. SNS dramatically increased FBC and reversed AA-induced changes without affecting the character of normal NVCs associated with bladder filling. Increased number of NVCs appears to be a consequence of increased fill time. Our finding that SNS does not interfere with normal filling contractions supports the contention that these NVCs are not reflexogenic in nature and that SNS does not interfere with normal bladder filling.

Funding: This study was funded by Neuromodulation Research, Medtronic, Inc., Minneapolis, MN.

Clinical Relevance: Neuromodulation

\section{Poster \# BS28}

\section{ASSESSMENT OF AUTONOMIC SENSORY FUNCTION WITH A NOVEL DEVICE IN THE MOUSE BLADDER}

Fuat Bicer, MD, PhD, Jin Young Kim, BS, Andrew Horowitz, MD, Firouz Daneshgari, MD, Guiming Liu, MD, PhD

Urology Institute, University Hospitals Case Medical Center, Case Western Reserve University, Cleveland, Ohio

(Presented by: Fuat Bicer, MD, PhD)

Introduction and Objectives: Various diseases can cause altered afferent autonomic sensitivity of bladder, including diabetes, neurologic or vascular lesions, multiple sclerosis, recurrent infection, interstitial cystitis and pelvic pain syndrome. In current urologic clinical practice, to study autonomic and sensory bladder neuropathy, clinicians still rely on patients' self-reported bladder sensation during the filling phase of urodynamics. We developed and tested the efficacy of an implantable bladder device (Fig.) which, when combined with the Neurometer ${ }^{\mathbb{R}}$, can be used to assess fiber specific afferent bladder sensation in the mouse. 
Materials and Methods: We developed an implantable bladder device that applies selective nerve fiber stimuli $(250 \mathrm{~Hz}$ for small myelinated A $\delta$ fibers and $5 \mathrm{~Hz}$ for unmyelinated $\mathrm{C}$ fibers) to the bladder mucosa in the mouse to determine bladder current perception threshold (CPT) values. We performed 4 experiments in $64 \mathrm{C} 57 \mathrm{BL} / 6 \mathrm{~J}$ mice to examine the effects of our device on voiding habits, assess the interobserver reliability of the sensory perception threshold and determine the effects of intravesical administration of resiniferatoxin and lidocaine on the sensory perception threshold.

Results: Sensory perception threshold values obtained by 2 blinded, independent observers were not different from each other $(p=0.74382)$. CPT values obtained at the 2 stimulation frequencies remained constant for at least 6 days after device implantation. A significant increase in CPT values after resiniferatoxin instillation was noted at a stimulus frequency of $5 \mathrm{~Hz}$ $(p=0.01347)$, whereas intravesical lidocaine led to an immediate increase in SPT at $250(p=0.0003)$ and $5 \mathrm{~Hz}(\mathrm{p}=0.0001)$. The implantation led to an early decreased voided volume and increased frequency of voids at 4 days, although these parameters returned to close normal after 6 days. The implanted bladder histology reveals defect created by device and mild inflammatory changes in next areas.

Conclusions: Assessment of bladder afferent sensation with our newly developed device is feasible in mice. It provides CPTs that appear to be fiber-type selective for autonomic bladder afferent nerves.

Clinical Relevance: Urodynamics

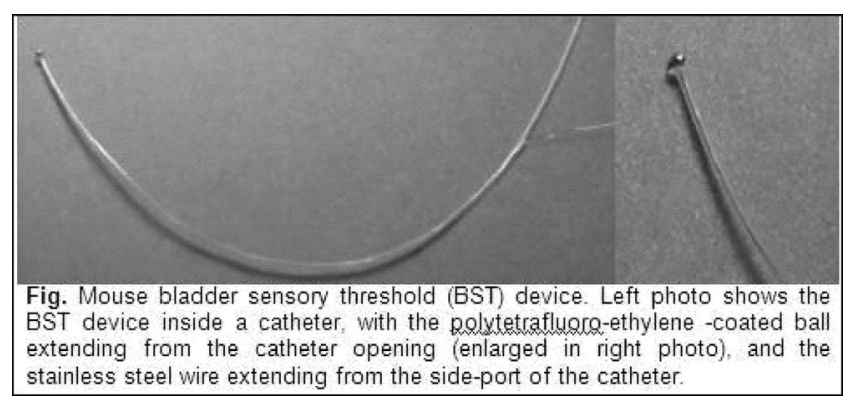

\section{Poster \# BS29}

BRAIN RESPONSE TO PELVIC FLOOR MUSCLE SOUEEZES: USING FUNCTIONAL NEAR INFRARED SPECTROSCOPY WITH URODYNAMICS

Becky Clarkson, PhD, Theodore Huppert, PhD, Nancy Beluk, BS, Stasa Tadic, MD, Neil Resnick, MD, Werner Schaefer, DI University of Pittsburgh

(Presented by: Becky Clarkson, PhD)

Introduction: Research of the brain's role in lower urinary tract function is important; however, restrictive brain scanning environments limit simultaneous bladder testing, and evaluation of individuals is difficult. Near infrared spectroscopy (NIRS) has potential to measure individual brain activity during bladder tests, without physical restrictions of a traditional scanner. NIRS can measure motor areas associated with urinary sphincter control from fMRI studies. We report on feasibility of NIRS to measure brain activity during pelvic floor muscle squeezes.

Methods: 25 urge incontinent and 9 continent women over age 60 were recruited to have urodynamic studies (UDS) with simultaneous measurement of brain activity using NIRS, targeting the supplementary motor area (SMA). Measurements of change in oxyhemoglobin $(\mathrm{HbO})$ concentration were made continuously during two sets of five pelvic floor muscle contractions (5s duration with 20 s rest period). HbO measurements were visually inspected to assess association with timing of squeezes. Since an advantage of NIRS is real time capability, visual assessment is important. Activity was confirmed if there were 4-5 peaks in the same frequency as the squeezes, within 10 seconds of the onset of the squeeze, for both sets of 5 squeezes.

Results: During pelvic floor muscle squeezes, temporally correlated activity in at least one channel of the SMA was observed in 13/25 incontinent and 4/9 control subjects. Only $16 \%$ of all SMA channels measured showed squeeze response; $51 \%$ showed no response; others were too noisy to assess. In all subjects with SMA activity, there was measurable urethral pressure increase. Block averaged data over all 10 squeezes per subject showed normals had significant, consistent bilateral SMA activation $(p<0.001)$. The incontinent group had some right SMA activation, but the left was deactivated or insignificant.

Conclusion: The aim was to assess NIRS' consistency across subjects. Although this method shows promise-response to motor tasks can be seen in the brain-inconsistency of measurements between channels (and absence of signal in a large proportion of channels) suggests that the experimental technique needs development.

Clinical Relevance: Female Urology-including Incontinence

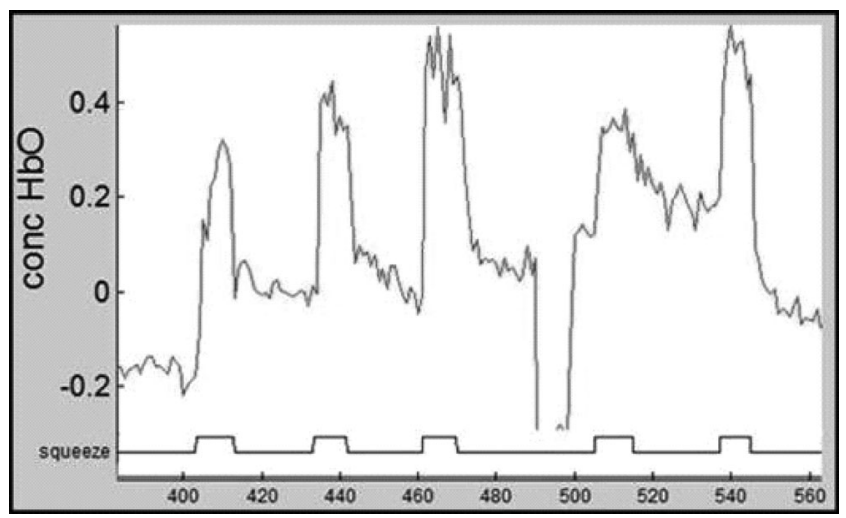

\section{Poster \# BS30}

\section{PELVIC ORGAN PROLAPSE ASSOCIATED ALTERATIONS OF SPHINGOSINE-1-PHOSPHATE/RHO-KINASE EXPRESSION IN HUMAN APICAL VAGINAL WALL}

Sang Ho Rhee, $\mathrm{MD}^{1}$, Ping Zhang, $\mathrm{PhD}^{1}$, Saifuddin Mama, $\mathrm{MD}^{1}$, Ricardo Caraballo, $\mathrm{MD}^{1}$, Robert Seftel, $\mathrm{BS}^{1}$, Adam Holzberg, $\mathrm{DO}^{1}$, Allen Seftel, $\mathrm{MD}^{1}$, Karolynn Echols, $\mathrm{MD}^{1}$, Michael DiSanto, $\mathrm{PhD}^{2}$

${ }^{1}$ Cooper University Hospital, Camden, $N J ;{ }^{2}$ Cooper Medical School of Rowan University

Introduction and Objectives: Pelvic organ prolapse (POP) is a debilitating condition affecting more than $50 \%$ of women over age 40 , yet the molecular basis for POP is not fully elucidated. The sphingosine-1-phosphate (S1P) cell signaling pathway regulates cell apoptosis and we have provided novel data that it also regulates contractility of SM in the lower urogenital system via the RhoA/Rho-kinase pathway. Thus, the objective of our study was to determine whether the expression of elastic fiber proteins and/or major components of the S1P/RhoA/Rhokinase signaling pathway are altered in the vagina of women with POP. 
Methods: Full thickness anterior vaginal wall specimens were obtained near the vaginal apex from women undergoing hysterectomy for benign gynecologic conditions other than POP nor stress urinary incontinence $(n=7)$ and from women having pelvic reconstructive surgery for POP $(n=9)$. Samples were divided into three strips and fresh tissue was processed for $\mathrm{H}$ \& $\mathrm{E}$ histological staining while frozen tissue was used for total RNA and protein extraction followed by Real-Time PCR and Western blotting.

Results Obtained: Histological staining confirmed that full thickness specimens were obtained from both groups. The mRNA expression of lysyl oxidase genes LOX \& LOXL1 and fibulin 5 (FBLN5) were decreased by 53\%, 72\% and 94\% while the three major S1P receptors (S1P1, S1P2, S1P3) were increased by 6.71-, 6.34-, 7.78-fold when normalized to expression of glyceraldehyde-3-phosphate dehydrogenase in women with POP compared to controls. In addition, mRNA expression of RhoA and the two Rho-kinase isoforms $\mathrm{ROK} \alpha$ and $\mathrm{ROK} \beta$ increased by 3.06-, 2.31-, 2.18-fold in women with POP compared to controls. Western blot confirmed that the S1P pathway was present in all samples.

Conclusions: In summary, the down-regulation of EFAP constituent mRNA correlates with the decrease elasticity observed by others and would be predicted to impair vaginal wall contractility. The increase in mRNA expression of the three S1P receptors accompanied by an increase in the RhoA/Rhokinase expression predicting increased calcium sensitization may represent a compensatory response to maintain force. In addition, since increases in S1P have been linked to decreased apoptosis, this might possibly suggest an additional compensatory role for this pathway. Taken together, our data suggests the S1P/RhoA/Rho-kinase pathway as a novel area for future $\mathrm{POP}$ research and therapeutic development.

\section{Poster \# BS31}

\section{PUBLICATION RATES OF PODIUM PRESENTED ABSTRACTS AT SUFU MEETINGS 2008-2013}

Natasha Ginzburg, MD, Darlene Morrissey, DO, Peter O'Hare, MD, Kristene Whitmore, MD Drexel University College of Medicine, Philadelphia, PA (Presented by: Natasha Ginzburg, MD)

Introduction and Objectives: The annual Society of Urodynamics, Female Pelvic Medicine, and Urogenital Reconstruction (SUFU) meeting and journal attract thousands of urologists, urogynecologists, researchers and allied health professionals focused on treatment and study of voiding dysfunction, neurourology, female urology, pelvic floor dysfunction and related topics. Over one hundred abstracts are presented yearly, in the form of podium presentation, moderated or nonmoderated poster presentation. No previous studies have examined the rate of publication of these sessions in peer reviewed journals.

Methods: All abstracts presented at the SUFU annual meetings from 2008 to 2012 were surveyed. Previously, similar studies have found oral podium presentations to have the highest yield of publications, so focus was on these presentations. Publication status as of October 2013 was assessed via MEDLINE. Abstracts were considered published if at least one author and at least one conclusion in the presented abstract were included in the published paper. Publication rates, time to publication, methodology and journal impact factor (JIF) were calculated. Results: Of the total 612 abstracts presented, 140 were podium presentations. Of these, 80 (42\%) have gone on to publication in peer reviewed journals. All but one of the abstracts originated from academic institutions. The rates of publication vary per year, with ranging from 13 of 40 (32.5\%) in 2012 to 23 of 46 $(50 \%)$ in 2010 . The average time to publication was $17.4(+/-$ 12) months, with the longest average time related to abstracts from $2008(22+/-17)$ compared to abstracts from 2012 (12.8 $+/-5.7)$. Abstracts were published in 17 journals; the average JIF was 3.157.

Conclusions: The rate of publication from podium presentation at the annual SUFU meeting over the last five years was slightly less than half. This is similar to published results from other national meetings. The average JIF was slightly higher than other national meeting studies. Further research into the nature of material (positive versus negative outcomes) and inclusion of poster presentation abstracts may add valuable information as to the character of research that is published. Clinical Relevance: Female Urology-including Incontinence

\section{Poster \# BS32}

\section{COMBINATION PHARMACOTHERAPY FOR TREATMENT OF NEUROGENIC BLADDER}

Jessica C. Lloyd, $\mathrm{MD}^{1}$, Ngoc-Bich Le, $\mathrm{MD}^{1}$, John S. Wiener, $\mathrm{MD}^{1}$, Paul C. Dolber, $\mathrm{PhD}^{2,3}$ and Matthew O. Fraser, $\mathrm{PhD}^{1,3,4}$

${ }^{1}$ Division of Urologic Surgery, Duke University Medical Center, Durham, NC; ${ }^{2}$ Division of Surgical Sciences, Duke University Medical Center, Durham, NC; ${ }^{3}$ Durham Veterans Affairs Hospital, Durham, NC; ${ }^{4}$ Institute for Medical Research, Durham, NC

(Presented by: Jessica C. Lloyd, MD)

Introduction and Objectives: Antimuscarinics are the mainstay of pharmacotherapy for neurogenic detrusor overactivity (NDO); however, bothersome side effects are common, including dry mouth, constipation and even cognitive impairment. The use of pharmacological combination approaches to treat symptoms of NDO may afford the opportunity to utilize lower doses of each individual drug, allowing achievement of the same or better clinical benefit with fewer side effects. We sought to determine whether combination therapy would improve indices of detrusor hyperreflexia in a rat model of chronic spinal cord injury (SCI).

Methods: After $\sim 60$ minutes of control open cystometry and 3 vehicle administrations, dose-response relationships were constructed in awake, restrained chronic suprasacral SCI rats ( $n=8-9$ /group, $\geq 4$ weeks post-SCI) using $1 / 2$ log increments of fesoterodine (antimuscarinic; F), doxazosin ( $\alpha 1$-adrenergic antagonist; D), CL-316,243 ( $\beta 3$-adrenergic agonist; $C L)$ and pregablin ( $\alpha 2 \delta$ subunit modulator of $\mathrm{N}$-type Ca2+ channels; $\mathrm{P}$ ) as either monotherapies or in paired combinations (10 groups total). Vehicles and drugs were given at $\sim 30$ minute intervals. Bladder capacity (BC), filling compliance (FC) and non-voiding contraction (NVC) count (\#) and maximum amplitudes (MA) were captured and measured using PowerLab 16SP. Data were analyzed by Friedman test and 2-way ANOVA, $\mathrm{P}<0.05$ was significant. All results are reported as means of data normalized to last vehicle.

Results: All monotherapies resulted in significant increases in BC (22-69\%), but were not different than each other. All combinations, except D $+\mathrm{F}$, resulted in significant $\uparrow$ in $\mathrm{BC}$ (39$180 \%) ; \mathrm{CL}+\mathrm{P}(116 \%)>\mathrm{CL}$, Dox, $\mathrm{P}, \mathrm{P}+\mathrm{D}$, and F+D; CL + F $(126 \%)>C L, P$ and $D+F$. Only $C L+P$ resulted in a significant increase in FC (115\%). NVC Max Amp was significantly by all treatments $(38-68 \%$ ) except $\mathrm{D}, \mathrm{P}, \mathrm{P}+\mathrm{D}$, and $\mathrm{P}+\mathrm{F}$ (latter 
approached at $\mathrm{P}<0.10)$; effect of $\mathrm{CL}+\mathrm{F}(68 \%)>\mathrm{D}$ and $\mathrm{P} ; \mathrm{CL}+\mathrm{D}$ $(54 \%)>$ P. NVC \# was significantly by D (52\%) and CL (47\%).

Conclusions: Monotherapies were effective on hallmark characteristics of neurogenic bladder, including BC (all), NVC Max Amp (CL, F) and NVC \# (CL, D). Combination therapies provided superior efficacy for $B C(C L+F, C L+P), F C(C L+P)$, and NVC Max Amp (CL $+F, C L+D)$. These results suggest that combination pharmacotherapy may provide similar or superior efficacy with reduced side-effect profiles for the treatment of neurogenic bladder.

Funding: Funded by Pfizer, Inc., USA.

Clinical Relevance: Neurogenic Bladder

\section{Poster \# BS33}

\section{INHIBITION OF P2 $\times 7$ RECEPTORS AFTER DORSAL-HORN TRANSECTION IMPROVES NEUROGENIC BLADDER DYSFUNCTION IN FEMALE RATS}

Timothy Boone, $\mathrm{MD}, \mathrm{PhD}^{1}$ and Alvaro Munoz, $\mathrm{PhD}^{2}$

${ }^{1}$ Houston Methodist Hospital, HMRI and VA-Medical Center, Houston, TX; ${ }^{2}$ Houston Methodist Research Institute, Houston, TX

(Presented by: Alvaro Munoz, PhD)

Introduction and Objectives: Bladder dysfunction is a common complication affecting patients with spinal cord injury (SCI), making restoration of efficient micturition a clinical priority. Animal models of regeneration after SCI suggest that amelioration of damage at the $\mathrm{SCI}$ site could improve visceral function and urine storage, but the extent of bladder sensory restoration remains unclear. In addition to promoting locomotor recovery, our main objective was to determine whether the specific inhibition of P2 $\times 7 \mathrm{R}$ after a SCI decrease the levels of nerve growth factor (NGF) in the bladder.

Methods: Female Sprague-Dawley rats were implanted with subcutaneous osmotic pumps filled with either saline or the P2 $\times 7$ antagonist brilliant blue-G (BBG). A continuous infusion of $30 \mu \mathrm{g} / \mathrm{kg} / \mathrm{hr}$ was started at the time of performing a dorsalhorn transection at the T8/T9 level. Spinal cord expression of $\mathrm{P} 2 \times 7 \mathrm{R}$ and Iba1 (microglia marker) was verified with immunohistochemistry. Locomotor effects were determined with the Basso, Beattie and Bresnahan (BBB) score and the standing capacity index (standings on rear paws in one minute) at $0,1,7,14$ or 28 days. After manual bladder expression, urine samples were stored for determination of residual volume (ml); creatinine (Cr; Jaffe' reaction); and NGF (ELISA) at 0, 1, 7 or 28 days. Cystometry was done via a suprapubic catheter in urethane anesthetized rats. Data were analyzed with ANOVA and Tukey's post test. Values are mean $+/-$ SEM. $\mathrm{P}<0.05$ was considered statistically significant.

Results: SCI increased the number of $\mathrm{P} 2 \times 7 \mathrm{R}+$ cells in the rostral \& caudal (to the SCI site) dorsal-horn area, while promoting the appearance of Iba1+ microglia. Systemic BBG treatment accelerated locomotion recovery while significantly improving standing capacity. The residual urine was $0.19+/-$ $0.03 \mathrm{ml}$ in intact rats $(n=6), 1.22+/-0.15 \mathrm{ml}$ in SCI rats $(n=6$; $\mathrm{p}<0.01$ vs intact) and $0.23+/-0.07 \mathrm{ml}$ in BBG-treated SCI animals $(\mathrm{n}=2)$. Urine NGF levels (pg NGF/mg Cr) were $45.8+/-$ 7.1 in control rats; $568.8+/-138.4$ in SCI-animals $(p<0.01$ vs intact), and $112.8+/-6.4$ in SCI rats receiving BBG $(p<0.01$ vs SCI rats). Cystometric studies showed a higher frequency of non-voiding contractions in SCI than in BBG-treated SCI rats. Conclusions: Inhibition of $\mathrm{P} 2 \times 7 \mathrm{R}$ at the SCI site may be a therapeutic target to improve bladder function by promoting neuroregeneration and decreasing urine NGF, a biomarker for neurogenic bladder.
Funding: Houston Methodist Foundation \& Brown Foundation Clinical relevance: Neurogenic Bladder

\section{Poster \# BS34}

\section{CONSTITUTIVELY ACTIVE HCN CHANNELS FACILITATE RELAXATION OF SPONTANEOUSLY CONTRACTING RAT BLADDER}

Mahendra Kashyap, PhD, Naoki Yoshimura, MD, PhD, Michael Chancellor, MD, Pradeep Tyagi, PhD

(Presented by: Alvaro Munoz, PhD)

Purpose: Involvement of cAMP signaling in the effect of $\beta 3-$ adrenoceptors (AR) agonist in urinary bladder relaxation is well established. In the present study we tested the hypothesis that basal levels of intracellular cAMP in bladder cells can constitutively activate the hyperpolarization-activated cyclic nucleotide-gated (HCN) channel activity. The interplay with cAMP can help characterize the function of HCN channels expressed in bladder.

Methods: Species-specific expression of HCN channels was studied in bladder tissues harvested from Sprague-Dawley rats of either sex and from human organ donors, using real-time OPCR, Western Blot and immunoreactivity. Effect of Lamotrigine and ZD7288 that are known to activate and cause nonspecific blockade of HCN channels, respectively was investigated on spontaneously contracting bladder strips in presence or absence of $\beta 3$-agonist $(C L 316,243)$ mediated detrusor relaxation.

Results: Expression of all four HCN channel subtypes (HCN1-4) was detected in separated tissue specimens of urothelium and detrusor from human as well as rat bladder relative to the subtype expression in heart of respective species (fig). Expression of HCN1 subtype was predominant in rat, whereas expression of HCN4 subtype was predominant in human bladder. Immunoreactivity and Western blot results confirmed the findings from mRNA measurements. Cumulative application of ZD7288 augmented the phasic contractions, whereas application of Lamotrigine (HCN channel activator) caused relaxation of spontaneously contracting rat bladder strips. Likewise, preincubation of strips with ZD7288 opposed the relaxant effect of both Lamotrigine and $\beta 3$ agonist CL316,243. Whereas, the CL316,243 mediated bladder relaxation was augmented following co-administration of Lamotrigine.

Conclusions: Expression of HCN channels in the bladder was noted with species-related differences in expression. Effect of ZD7288 (HCN channel blocker) demonstrated that constitutively active HCN channels counter the spontaneous detrusor contractility. Functional interaction of HCN channel activity in the action of $\beta 3 \mathrm{AR}$ agonist highlight a discovery of a new therapeutic target for addressing the detrusor contractility.

Clinical Relevance: LUTS / Voiding Dysfunction
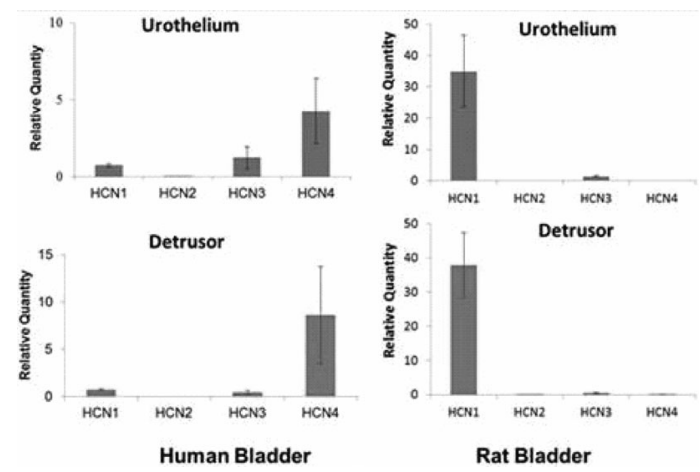


\section{Poster \# BS35}

PURINERGIC RESPONSES VIA THE ACTIVATION OF P2Y1 RECEPTORS IN MURINE DETRUSOR PDGFRALPHA CELLS

Haeyeong Lee, PhD, Byoung Koh, BS, Kenton Sanders, PhD, Sang Don Koh, MD, PhD

UNR

(Presented by: Haeyeong Lee, PhD)

Introduction and Objectives: Recently we discovered a new class of interstitial cell in the bladder and identified these cells with antibodies against platelet-derived growth factor receptor- $\alpha$ (PDGFR $\alpha)$. PDGFR $\alpha+$ cells have been identified in human, guinea pig and murine detrusor muscle. However, little is known about the molecular apparatus of PDGFR $\alpha+$ cells that might facilitate neural responses. Reports in the literature demonstrated purinergic relaxation responses in detrusor muscles: i) ATP induces transient contraction and prolonged relaxation of detrusor muscles and ii) P2Y receptor-mediated SK channel activation is hypothesized to be the mechanism for purinergic relaxation. These responses have been attributed to SK conductances in SMC. However, our previous findings demonstrated that the current density in PDGFR $\alpha+$ cells attributed to SK channels is far higher ( 100 times) than in SMC. Thus, we hypothesize that purinergic responses might be mediated by activation of P2Y receptors in PDGF $\alpha+$ cells.

Methods: We applied molecular and patch clamp techniques to bladder detrusor removed from PDGFR $\alpha+-$ eGFP+ mice (Pdgfratm11(EGFP)Sor/J mice). These novel mice express bright GFP in the nuclei of the PDGFRÿ!+ cells, thus making them identifiable from the population of enzymatically dispersed cells in the detrusor muscle. We analyzed the qualitative and quantitative expression of P2ry transcripts in FACS-sorted PDGFR $\alpha+$ cells. Effects of purines, P2Y receptor agonist and antagonist were examined in these cells using patch clamp approaches.

Results: We characterized the transcriptional expression of $\mathrm{P} 2 \mathrm{Y}$ receptors in unsorted, sorted SMCs and sorted PDGFR $\alpha+$ cells. PDGFRÿ!+ cells dominantly express P2ry1, P2ry2, P2ry4 and P2ry14 compared with unsorted cells or SMCs. ATP activated large outward currents which were inhibited by SK channel blockers (apamin and UCL1684). ATP also induced significant membrane hyperpolarization. P2Y1 agonist mimicked the ATP responses and P2Y1 antagonist inhibited ATP-activated SK currents. Furthermore, response of PDGFR $\alpha+$ cells to ATP in P2ry1-/- was significantly diminished. P2X receptor agonist had no effect on PDGFR $\alpha+$ cells but activated inward currents in SMCs.

Conclusions: Purines are coupled to activation of SK channels in PDGFR $\alpha+$ cells by binding to P2Y receptors (mainly P2Y1 receptor) and affect membrane stabilization and relaxation during bladder filling.

Funding: Supported by NIH/NIDDK R01 DK098388

Clinical Relevance: Neuromodulation

\section{Poster \# BS36}

FUNCTIONAL LINK BETWEEN MUSCARINIC RECEPTORS AND LARGE-CONDUCTANCE CA2 +-ACTIVATED K + CHANNELS IN FRESHLY-ISOLATED HUMAN DETRUSOR SMOOTH MUSCLE CELLS

Shankar Parajuli, $\mathrm{PhD}^{1}$, Kiril Hristov, $\mathrm{PhD}^{1}$, Oiuping Cheng, $\mathrm{PhD}^{1}$, John Malysz, $\mathrm{PhD}^{1}$, Eric Rovner, $\mathrm{MD}^{2}$ and Georgi Petkov, $\mathrm{PhD}^{1}$

${ }^{1}$ University of South Carolina, Columbia, SC; ${ }^{2}$ Medical University of South Carolina, Charleston, SC

(Presented by: Georgi Petkov, PhD)
Introduction and Objectives: Activation of muscarinic receptors (MRs) constitutes the primary mechanism for enhancing excitability and contractility of human detrusor smooth muscle (DSM). Since the large conductance Ca2+-activated $\mathrm{K}+(\mathrm{BK})$ channels are key regulators of human DSM excitability, we investigated whether MR activation increases human DSM excitability by inhibiting BK channels. The functional link between MRs and BK channels in human DSM cells has not been previously explored.

Materials and Methods: We used the MR agonist, carbachol, to determine the changes in BK channel activity upon MR activation in freshly-isolated human DSM cells obtained from open bladder surgeries using the perforated whole cell and single BK channel patch-clamp recordings.

Results: Carbachol inhibited the amplitude and frequency of BK channel-mediated spontaneous transient outward currents and spontaneous transient hyperpolarizations, which are triggered by ryanodine receptor $\mathrm{Ca} 2+$ release. Carbachol also caused membrane potential depolarization, which was not observed in the presence of iberiotoxin, a BK channel inhibitor, indicating a critical role of the BK channels. The potential direct carbachol effects on BK channels were examined under conditions of removing the major cellular Ca2+ sources for BK channel activation with pharmacological inhibitors, including thapsigargin, ryanodine and nifedipine. In the presence of these inhibitors, carbachol did not affect the single BK channel open probability and mean BK channel conductance (cellattached configuration) or the voltage-induced whole cell BK currents.

Conclusions: The present study for the first time revealed that activation of MRs suppresses BK channel-mediated spontaneous transient outward currents and spontaneous transient hyperpolarizations, and depolarizes human DSM cells. Thus, our findings strongly suggest that there is a functional link between the MRs and BK channels in human DSM cells. The data support the concept that MR activation triggers indirect functional BK channel inhibition mediated by intracellular $\mathrm{Ca} 2+$, thus increasing the excitability in native human DSM cells.

Funding: Supported by NIH RO1DK084284 to G.V. Petkov and by a fellowship from the Urological Care Foundation Research Scholars Program and the Allergan Foundation to S.P. Parajuli.

\section{Poster \# BS37}

\section{AGE DEPENDENT, DIFFERENTIAL REGULATION OF MUSCARINIC RECEPTORS BY CAVEOLAE IN BLADDER SMOOTH MUSCLE}

Vivian Cristofaro, $\mathrm{PhD}^{1}$, Hongying Cao, $\mathrm{MD}^{2}$, Subbarao V. Yalla, $\mathrm{MD}^{3}$ and Maryrose P. Sullivan, $\mathrm{PhD}^{4}$ ${ }^{1}$ Boston VA Healthcare System, Harvard Medical School; ${ }^{2}$ Boston VA Healthcare System Boston; ${ }^{3}$ VA Healthcare System Boston;

${ }^{4}$ A Healthcare System Boston

(Presented by: Vivian Cristofaro, PhD)

Introduction and Objectives: Detrusor contraction is mediated by muscarinic acetylcholine receptor (mAChR) activation, predominately by the M3 subtype. Despite the substantially greater M2 mAChR density in the detrusor, the functional role of M2 receptors remains unclear. Several G-protein-coupled receptors are thought to be regulated by caveolae, either by statically associating with caveolae or by translocating into or out of caveolae with ligand binding. Thus, these cholesterolrich membrane domains may compartmentalize, modulate and integrate signaling events at the cell surface to facilitate or 
impede cellular responses to extracellular stimuli. However, the role of caveolae in regulating mAChRs has not been well studied. This study investigates whether muscarinic contractile responses in the bladder are mediated by caveolae.

Methods: Bladder tissues from female rats (7-8 weeks or 18-22 weeks of age) were mounted in organ baths and equilibrated under resting tension at $37^{\circ} \mathrm{C}$. Carbachol (CCh) dose response curves (10-9-10-5M) and frequency response curves (electric field stimulation, under conditions of purinergic desensitization) were generated before and after treatment with $\mathrm{mAChR}$ antagonists 4-DAMP (10nM) or AFDX $(0.1 \mu \mathrm{M})$ to partially inactive M3 and M2 receptors, respectively. Subsequently, bladder tissue was exposed to methyl- $\beta$-cyclodextrin (mCD), an agent that disrupts caveolae by depleting membrane cholesterol, and CCh response or EFS response curves were repeated.

Results: The contractile response to CCh (in the absence of antagonists) was not affected by mCD treatment. In older rat bladders, the contractile responses to CCh and EFS were significantly enhanced by $\mathrm{MCD}$ in the presence of 4-DAMP (to unmask the M2 response), suggesting that caveolae inhibit M2 mediated signaling. In the presence of AFDX (to expose the M3 response), the contractile response to CCh was not affected by $\mathrm{mCD}$. In contrast, in younger animals, the contractile response to $\mathrm{CCh}$ was markedly attenuated by $\mathrm{mCD}$ in the presence of AFDX, suggesting an age dependent regulation of M3 receptors by caveolae.

Conclusions: These findings suggest that caveolae function as negative regulators of M2 receptor activated signaling. Thus the loss of caveolae may disinhibit M2 mAChR signaling processes and result in enhanced muscarinic contractile responses.

Funding: Supported by Medical Research Service, Department of Veterans Affairs, Washington, DC and Pfizer OAB Grant.

\section{Poster \# BS38 \\ INTERACTION OF TRPV4 AND SK3 CHANNELS AND THEIR FUNCTIONAL ROLE IN MURINE DETRUSOR PDGFRALPHA CELLS}

Haeyeong Lee, PhD, Byoung Koh, BS, Kenton Sanders, PhD, Sang Don Koh, MD, PhD

UNR

(Presented by: Haeyeong Lee, PhD)

Introduction and Objectives: Recently we reported the functional expression of SK channels in detrusor PDGFR $\alpha+$ cells. Interestingly this cell does not display voltage-dependent $\mathrm{Ca} 2+$ channels. Therefore it is important to find the source of $\mathrm{Ca} 2+$ influx for activation of SK channels. Among TRP channels, TRPVs are more Ca2+ permeable. In a previous paper, Trpv4-/mice demonstrated an increase in frequency of non-voiding contractions. The mechanism of this phenotype has not been studied. Thus, we hypothesized that one of TRPV channels is highly expressed in PDGFR $\alpha+$ cells and may be the source of Ca2+ influx to activate SK channels.

Methods: We applied molecular approaches, protein chemistry and patch clamp techniques using detrusor removed from PDGFR $\alpha+-$ eGFP + mice (Pdgfratm11(EGFP)Sor/J mice). The bright GFP in the nuclei of PDGFRÿ!+ cells was used for cell sorting after enzymatic dispersion. We analyzed molecular expression of TRP channels and quantified protein expression of TRPV channel from sorted cells. We also performed coimmunoprecipitation to test functional interaction between TRPV4 and SK3 channels. Effects of TRPV4 agonist and antagonist on the activity of SK channel were examined in PDGFRÿ!+ cells using patch clamp approaches.

Results: Quantitative analysis of PCR in Trp transcripts demonstrated Trpv4 was highly expressed in PDGFR $\alpha+$ cells compared to in smooth muscle cells. Western blot from sorted cells supported that TRPV4 was highly expressed in PDGFR $\alpha+$ cells but not in smooth muscle cells. Furthermore, coimmunoprecipitation suggested the protein interaction between TRPV4 and SK3 channels. In patch clamp experiments of PDGFRÿ!+ cells, TRPV4 agonist activated inward currents at a holding potential of $-80 \mathrm{mV}$. However, at positive potentials (e.g. at $-60 \mathrm{mV})$, TRPV4 agonist activated transient inward currents followed by outward currents. These outward currents were inhibited by SK channel blocker.

Conclusions: TRPV4 and SK3 channels are physically and functionally interacted in plasma membrane of PDGFR $\alpha+$ cells. Ca2+ influx through TRPV4 channels directly activates SK3 channels. Since TRPV4 are mechanosensitive channels, activation of TRPV4 during bladder filling activate SK3 channels and stabilize membrane potentials and prevent detrusor overactivity. A, Increase in frequency of non-voiding contraction in Trpv4-/- detrusor can be explained by the present findings.

Funding: Supported by NIH/NIDDK R01 DK098388

\section{Poster \# BS39}

\section{DETRUSOR SMOOTH MUSCLE-DEPENDENT ACUTE REGULATION OF HUMAN BLADDER COMPLIANCE}

MaryEllen T. Dolat, MD, Michael D. Byrne, MD, John E. Speich, PhD, R. Wayne Barbee, PhD, Paul H. Ratz, PhD and Adam P. Klausner, PhD Virginia Commonwealth University, Richmond, VA (Presented by: MaryEllen T. Dolat, MD)

Introduction: It is generally accepted that bladder compliance changes only through chronic disease processes. Our work on rabbit bladder challenges this model, revealing that detrusor length-dependent preload (a measure of compliance) is acutely regulated by detrusor smooth muscle (DSM). Because DSM is in-series with afferent sensory nerves, alterations in DSMdependent compliance regulation may have clinical consequences if such a system operates in human bladder. The present study was designed to examine this new model in human DSM (hDSM).

Methods: Strips of hDSM were obtained following cystectomy (male, age 25-62 y). First, an active length-tension curve identified the reference muscle length (Lref). Then tissues were subjected to 3 sequential load-unload cycles and tension (T) was measured at Lref for each cycle as an index of compliance (T1, T2, T3; see Fig for protocol). To prevent spontaneous contraction, load-unload cycles were performed in $0 \mathrm{mM} \mathrm{Ca} 2+$. Prior to cycles $1 \& 3$, tissues were contracted with $\mathrm{KCl}$ at $60 \%$ Lref to mimic the final stage of a voiding contraction. No contraction occurred in the $20 \mathrm{~min}$ period at $60 \%$ Lref between cycles 1 \& 2 . The rho kinase inhibitor $\mathrm{H}-1152(0.3 \mu \mathrm{M})$ was added to a set of tissues prior to cycle 3 .

Results: In hDSM, T2/T1 $=0.51$, which was $<1.0 \quad(\mathrm{n}=3, \mathrm{p}$ $<0.05$ ) because a fraction of T1 at Lref was lost to strain softening during loading to $130 \%$ Lref and was not restored during the wait at $60 \%$ Lref. T3/T1 $=0.90$, which was not $<1.0$, indicating that, as in rabbit DSM, tension at Lref lost during loading to $130 \%$ Lref was restored by contraction at $60 \%$ Lref. In tissues exposed to $\mathrm{H}-1152$ during contraction prior to cycle 3 , $\mathrm{T} 3 / \mathrm{T} 1=0.59$, which was not $>$ the $\mathrm{T} 2 / \mathrm{T} 1=0.46$ obtained prior to drug treatment $(n=3)$, indicating that rho kinase inhibition 
prior to T3 prevented restoration of tension lost to strain softening. These data show that hDSM, like rabbit DSM, displays length-history- and rho kinase-dependent compliance regulation.

Conclusion: As in rabbit, compliance in hDSM can be acutely regulated. Pathological increases in the sensitivity of this mechanism may accentuate bladder tone during filling suggesting a relationship to overactive bladder. Clinical Relevance: LUTS/Voiding Dysfunction

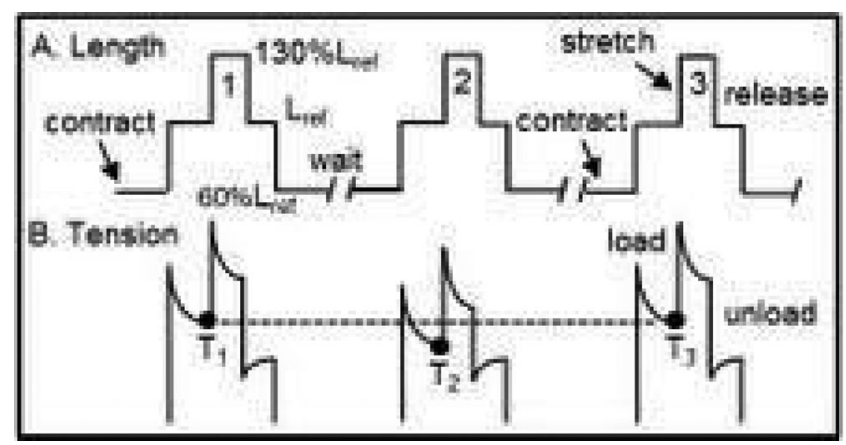

Poster \# BS41

SECRETOME OF MESENCHYMAL STEM CELLS FACILITATES RECOVERY FROM SIMULATED CHILDBIRTH INJURY

Kangli Deng, $M^{1,2}$, Danli Lin, $M^{1,2}$, Brett Hanzlicek, $M^{1,2}$, Brian Balog, $\mathrm{BS}^{1,2}$, Hui Zhu, $\mathrm{MD}, \mathrm{PhD}^{2}$, Zhiquan $\mathrm{Hu}, \mathrm{MD}^{3}$, Zhangqun Ye, $\mathrm{MD}^{3}$ and Margot Damaser, $\mathrm{PhD}^{1,2,4}$

${ }^{1}$ Dept. of Biomedical Engineering, Cleveland Clinic, Cleveland, $\mathrm{OH} ;{ }^{2}$ Louis Stokes Cleveland Veterans Affairs Medical Center, Cleveland, $\mathrm{OH} ;{ }^{3}$ Dept. of Urology, Tongji Hospital, Huazhong University of Science and Technology, Wuhan, China; ${ }^{4}$ Glickman Urological and Kidney Institute, The Cleveland Clinic, Cleveland, $\mathrm{OH}$

(Presented by: Kangli Deng, MD)

Introduction and Objectives: Simulated childbirth injury, including vaginal distension (VD) and pudendal nerve crush (PNC), results in decreased leak point pressure (LPP), indicative of stress urinary incontinence (SUI) in rats. Mesenchymal stem cells (MSCs) have been shown to facilitate repair after injury via paracrine secretions. The aim of this study was to investigate the effects of a single treatment with concentrated conditioned media (CCM) cultured from MSC, containing the MSC secretome, on the recovery from simulated childbirth injury.

Methods: Sixty adult Sprague-Dawley female rats (250-300g) were allocated into 3 groups: sham injured with concentrated control media $(n=20)$, PNC + VD with concentrated control media $(n=20)$, and PNC + VD with CCM $(n=20)$. CCM was obtained by incubating rat MSCs in serum-free media for 24 hours. The cultured MSC supernatant was extracted and concentrated 50 times. Each animal received 300ul of CCM or media concentrated using the same process ip 1 hour after either PNC + VD or sham injury. Three weeks after injury, 14 rats in each group underwent LPP testing with simultaneous external urethral sphincter (EUS) electromyography (EMG) and pudendal nerve sensory branch potential (PNSBP). The other 6 rats in each group were used for histology. Quantitative outcomes were analyzed with a one way ANOVA with $p<0.05$ indicative of statistically significant differences.

Results: LPP was significantly decreased 3 weeks after PNC + VD treated with control media compared to sham injured animals. LPP was not significantly different after PNC + VD when treated with CCM compared to the other 2 groups. PNSBP was significantly decreased 3 weeks after PNC + VD treated with control media compared to sham injured animals but not when treated with CCM. There were no significant differences between the groups in EUS EMG outcomes. Qualitative histological assessment demonstrated an increase in elastic fibers and a change in their orientation around the EUS 3 weeks after PNC + VD treated with either control media or CCM with a noticeable increase in elastic fibers in animals treated with CCM.

Conclusion: Functional recovery from PNC + VD was improved with CCM treatment potentially due to an increase in elastin fibers in the urethra. Cell-free derivatives, such as CCM, may have potential as clinical therapy to facilitate recovery from childbirth and prevent or ameliorate development of SUI.

Funding: Dept. of Veterans Affairs and the China Scholarship Council

Clinical Relevance: Female Urology-including Incontinence

\section{Poster \# BS42}

\section{RATIONAL DESIGN OF 3-D NANOSCAFFOLDS FOR} ENGINEERED POP SOFT TISSUE DESIGN

Johnny Yi, $\mathrm{MD}^{1}$, Christine Pauken, $\mathrm{PhD}^{2}$, Vincent Pizziconi, $\mathrm{PhD}^{2}$, Stephen Massia, $\mathrm{PhD}^{2}$ and Jeffrey Cornella, MD $^{3}$

${ }^{1}$ Mayo Clinic Arizona, Phoenix, AZ; ${ }^{2}$ Arizona State University, Tempe, AZ; ${ }^{3}$ Mayo Clinic Arizona

(Presented by: Johnny Yi, MD)

Introduction and Objectives: Several factors contribute to failure following pelvic reconstructive surgery including suboptimal mechanical properties of implant materials, tissue degradation induced by implant and lack of regenerative capacity. Vaginal fibroblasts are crucial for producing extracellular matrix proteins that provide structural support. We hope to address these modes of failure with a tissue engineered implant. We focus on vaginal fibroblasts seeded onto an electrospun scaffold to create a tissue engineered implant for use during reconstructive surgery.

Methods: Electrospinning of a Dextran poly-acrylic acid polymer solution mimics the nanoscale of ECM within the vaginal tissue. Thermal crosslinking allows the formation of ester bonds to delay degradation and allow seeding of cells. 5 prolapse and 5 nonprolapse patients were recruited and tissue collected from the anterior vagina during hysterectomy. Fibroblasts were isolated and cultured, resulting in 7 stable cell lines. Fibroblasts were seeded onto electrospun scaffold and cultured for 4-8 days before fixation and immunohistochemistry(IHC).

Results: IHC was completed and confirmed fibroblast cell type. IHC was also performed for collagen I and III subtypes. Following thermal crosslinking, vaginal fibroblasts were seeded onto scaffold. Assays showed active mitoses. IHC of the seeded cells showed continued expression of FSP, Collagen I and III. Scaffold seeded fibroblasts show rounded cells as compared to spindle shaped cells grown on plates. Further characterization of the scaffold, biomechanical testing and western blot experiments are in progress.

Conclusions: Recurrence following prolapse surgery leads to a significant number of surgical interventions each year. Augmentation with vaginal mesh has led to scrutiny due to patient morbidity. There is a biomechanical mismatch between the synthetic mesh and the vagina. This may lead to poor wound healing. Rational design of a scaffold may provide a micro- 
environment that more closely matches the native tissue in mechanical properties and composition. This microenvironment may promote normal wound healing. Careful design of an implant on a cellular and biomechanical level is necessary to optimize graft material. Our preliminary studies show a favorable morphology of vaginal fibroblasts when seeded onto the scaffold with continued expression of key proteins. Further, the observed morphology suggests a less contractile response of the fibroblasts as compared to fibroblasts grown on cell culture plates.

Clinical Relevance: Pelvic Organ Prolapse.

\section{Poster \# BS43}

\section{PERIURETHRALLY IMPLANTED AFFINITY-BASED CCL7} RELEASING HYDROGELS FOR STIMULATING STEM CELL MIGRATION IN STRESS URINARY INCONTINENCE (SUI)

Zhina Sadeghi, $\mathrm{MD}^{1,2}$, Edgardo Rivera ${ }^{3}$, Janathan Kenyon, $\mathrm{PhD}^{1,2}$, Sapna Satyanarayan ${ }^{1,2}$, Nick Wang, $\mathrm{PhD}^{3}$, Mike Kavran, $\mathrm{MS}^{1,2}$, Amin Rabie ${ }^{1,2}$, Joseph Molter ${ }^{4}$, Chris Flask, $\mathrm{PhD}^{4}$, Firouz Daneshgari, $\mathrm{MD}^{1,2}$, Horst von Recum, $\mathrm{PhD}^{3}$ and Adonis Hijaz, $\mathrm{MD}^{1,2}$

${ }^{1}$ Urology Institute, University Hospitals Case Medical Center;

${ }^{2}$ Department of Urology, Case Western Reserve University School of Medicine, Cleveland, $\mathrm{OH}{ }^{3}$ Department of Biomedical Engineering, Case Western Reserve University, Cleveland, $\mathrm{OH}$; ${ }^{4}$ Department of Radiology, Case Western Reserve University, Cleveland, $\mathrm{OH}$

(Presented by: Zhina Sadeghi, MD)

Introduction: Chemokine ligand 7 (CCL7) is over expressed in urethrovaginal tissues following induction of SUI in a rat model simulating birth trauma. CCL7 over expression has shown potency for stimulating targeted stem cell migration. Our aim is to prepare affinity-based controlled loading/release CCL7 hydrogels, to provide therapeutic levels of CCL7 to stimulate host stem cell migration.

Methods: Bovine serum albumin (BSA) hydrogels were synthesized by mixing BSA/heparin solution with EDC crosslinker. Poly-Ethylene Glycol (PEG) gels were formed by adding PEG solution to the chilled heparin sodium/EDC/NHS. CCL7 (200ug/ $\mathrm{ml}$ ) was loaded into the hydrogels. Daily CCL7 release profiles were characterized in vitro for 6weeks and measured by CCL7 ELISA. To measure CCL7 biodistribution, 12 female SD rats were implanted periurethrally with BSA-heparin or PEG-Heparin hydrogels (w/wo CCL7). CCL7 level was measured in urethrovaginal tissue and blood by ELISA 12 days after. In 4 rats implanted with BSA hydrogel (w/wo CCL7), Lavacell labeled human Mesenchymal Stem Cells (hMSCs) were periurethrally injected, followed by fluorescent cryoimaging $24 \mathrm{hrs}$ after.

Results: Figure shows affinity hydrogels were able to maintain a 4 fold difference in concentration over non affinity polymers. CCL7 hydrogels were found 3 weeks post-implantation, with no tissue disruption. Blood CCL7 concentration was below the detectable range. CCL7 local distribution was the highest for PEG gels with up to 300 times the content of CCL7 than vehicle only. Lavacell labeled hMSC showed a diffuse distribution away from the injection site in control hydrogel recipient rats, but a highly localized intense signal in CCL7 hydrogel rats.

Conclusions: Affinity-based system may be used for sustained release of CCL7. Hydrogels demonstrated feasible embedding and durability in-vivo. While our cumulative release data would predicts higher amount of CCL7 release from BSA gels, PEG gels had up to 60 times higher local concentrations of CCL7 in-vivo. Successful controlled CCL7 mediated stem cell homing to the lower urinary tract would introduce the potential for non-operative treatment or prevention of SUI.

Funding: NIH/NIDDK 5K08DK090134 to A. Hijaz.

Clinical Relevance: Female Urology-including Incontinence

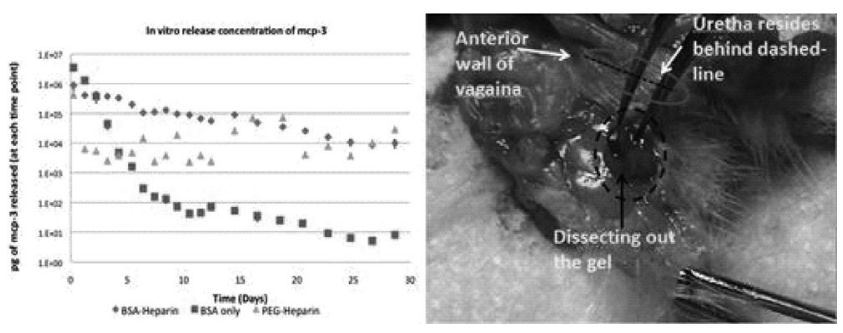

Poster \# BS44

LOCALIZED URETHRAL OVEREXPRESSION OF CCL-7 INDUCES MSC RETENTION AT SITES OF TISSUE INJURY

Jonathan Kenyon, $\mathrm{PhD}^{1}$, Zhina Sadeghi, $\mathrm{MD}^{1}$, Sapna Satyanarayan, $\mathrm{BS}^{1}$, Lauren Byrne, $\mathrm{MD}^{1}$, Kerry Grimberg, $\mathrm{PhD}^{1}$, Zhenghong Lee, $\mathrm{MD}^{2}$, Ahmet Ozer, $\mathrm{MD}^{3}$, Michael Kavran ${ }^{1}$, Cengiz Altuntas, $\mathrm{MD}^{1}$, Cagri Sakalar, $\mathrm{MD}^{4}$, Firouz Daneshgari, $\mathbf{M D}^{1}$ and Adonis Hijaz, $\mathbf{M D}^{1}$

${ }^{1}$ Department of Urology, University Hospitals, Case Western Reserve University, Cleveland, $\mathrm{OH} ;{ }^{2}$ Department of Radiology, University Hospitals, Case Western Reserve University,

Cleveland, $\mathrm{OH} ;{ }^{3}$ Department of Genetics, Case Western Reserve University, Cleveland, $\mathrm{OH} ;{ }^{4}$ Department of Immunology, Lerner Research Institute, Cleveland Clinic, Cleveland, $\mathrm{OH}$

(Presented by: Jonathan Kenyon, PhD)

Introduction and Objectives: Monocyte chemoattractant protein-3 (MCP-3), encoded by the CCL-7 gene in both rodents and humans, is a stem cell homing factor. MCP3 expression is observed in tissue injury and has shown promise as a regenerative trigger for local MSC at sites of injury. In an effort to improve postpartum stress urinary incontinence (SUI) we are investigating the feasibility, in animal models, of temporal overexpression of MCP3 protein in urethral and perivaginal tissue as a therapeutic option for women with established SUI. Hypothesis: MCP3 protein induces MSC retention at sites of urethral tissue damage.

Results: Mouse MCP3 (mMCP3) expressing plasmid (pORF5mMCP3) injected into perivascular space tissue harvested in 15 animals at 1, 3 and 5 weeks post-injection; mMCP3 expression was assessed in mouse tissue and sera by ELISA. Increased mMCP3 expression was observed in serum, bladder, and heart tissue at 5 weeks post-injection. Kidney, liver, lung and ovary tissue did not have increased $\mathrm{MMCP} 3$ expression compared to controls. Initially $\mathrm{mMCP} 3$ expression was observed in uterine tissue however, after the 3 weeks no difference was observed between plasmid and controls. To show MCP3 is necessary for hMSC retention in injury, wild type and CCL-7-\u8722? deficient mice were injected with LavaCell reporter dye labeled hMSC. Animals were then sacrificed at $24 \mathrm{~h}$, flash frozen, sectioned and imaged. We observed hMSC retention exclusively in the periurethral space of wild type mice. In contrast however, hMSC injected into CCL-7-\u8722? animals remained dispersed along the needle track. In a future experiment, LavaCell labeled hMSC will be injected into CCL-7 deficient mice treated with $\mathrm{hMCP} 3$ expressing plasmid in order to demonstrate return to CCL-7 wild type hMSC retention in CCL-7 deficient mice after hMCP3 supplementation. Finally, in order to show human MCP3 (hMCP3) improves SUI, we induced SUI in rats by vaginal distention (VD) followed immediately by 
hMCP3 expression plasmid perivaginal injection; leak point pressure was then assessed at 4 days. Analysis of leak point pressure in VD animals treated with hMCP3 expression plasmid is ongoing.

Conclusion: Current therapeutic options for the treatment of SUI are limited. Our work demonstrates the utility of localized expression of MCP3 at sites of urethral injury through hMSC retention. We argue new treatments based on MCP3 and hMSC will be less invasive and superior compared to current standard of care outcomes.

Clinical Relevance: Female Urology-including Incontinence

\section{Poster \# BS45}

\section{EFFECT OF HUMAN ADIPOSE MESENCHYMAL STEM CELLS ON THE CONTINENCE MECHANISM AND HISTOLOGICAL ANALYSIS IN A VAGINAL DISTENTION RAT MODEL OF STRESS URINARY INCONTINENCE}

Rebecca James, MD, Zhina Sadeghi, MD, Fuat Bicer, MD, Jack Weaver, BS, Michael Kavran, MS, Ramzi El Hasan, BS, Hooman Soltanian, MD, Adonis Hijaz, MD

Cleveland, $\mathrm{OH}$

(Presented by: Rebecca James, MD)

Introduction and Objectives: Stress urinary incontinence (SUI) is a bothersome disease affecting women. It can arise as the result of connective tissue damage and resultant laxity of the supporting ligaments of the pelvis, much of which can be sustained after birth trauma. After having previously shown a significant return of continence with hASCs treatment in a vaginal distention (VD) rat model of SUI, this study examined the histological effects after treatment with hASCs.

Methods: Female Sprague-Dawley rats $(\mathrm{N}=90)$ underwent serial VD with post-operative periurethral injections with $0.1 \mathrm{~mL}$ of either saline or hASCs. One group (control) did not undergo VD. Leak point pressures (LPP) were measured at 4, 14, and 42 days after VD treatment. LPP was measured in control rats (no VD or treatment; $n=15$ at one time point. Significance between groups was determined using a two-tailed unpaired t-test assuming equal variance and ANOVA with a post-hoc Tukey test, $\mathrm{P}<0.05$. The controls were compared to each time point $(4,14$, and 42 days) with Dunnett's multiple comparison test. Urethrovaginal tissues were harvested and immunohistochemistry performed with Masson's Trichrome ${ }^{\mathrm{TM}}$ and hematoxylin-eosin (morphology), Fast Myosin Skeletal Heavy Chain $^{\text {TM }}$ (skeletal muscle), and Verhoeff Van Gieson Elastin Stain $^{\text {TM }}$ (elastin). Analysis was performed of tissue of interest/ whole tissue ratios using Kruskal-Wallis rank of sums and chisquared tests.

Results: Previously reported results included a statistically significant increase in LPP in the group treated with hASC (22.22 mmHg, SD 4.391) $(\mathrm{P}=<.0001)$ compared to the saline and control groups. Elastin was significantly increased in the tissues of the hASC-treated group $(\mathrm{P}=0.0053)$. No significant differences existed between the groups in immunohistochemistry analysis of skeletal muscle or morphologies.

Conclusion: This study demonstrated a trend toward improved recovery of continence by days 4,14 and 42 after VD with hASC treatment compared to saline as demonstrated by mean LPPs. Connective tissue (including elastin) plays a significant role in tissue recovery with returned continence after hASC administration. These findings could indicate a translational role for hASC administration after vaginal birth trauma in humans with further investigations.

Clinical Relevance: Female Urology-including Incontinence

\section{Poster \# BS46}

\section{EFFECTS OF UROTHELIAL P2 $\times 3$ RECEPTOR INHIBITION ON} RAT BLADDER CONTRACTILITY

Andrew Ferguson ${ }^{1}$, Broderick Sutton ${ }^{1}$, Timothy Boone, $\mathrm{MD}, \mathrm{PhD}^{2}$, Anthony Ford, $\mathrm{PhD}^{3}$ and Alvaro Munoz, $\mathrm{PhD}^{1}$

${ }^{1}$ Houston Methodist Research Institute, Houston, TX; ${ }^{2}$ Houston Methodist Hospital, MHRI and VA-Medical Center, Houston, TX; ${ }^{3}$ Afferent Pharmaceuticals, San Mateo, CA

(Presented by: Alvaro Munoz, PhD)

Introduction and Objectives: Bladder ATP activates $\mathrm{P} 2 \times 1 \mathrm{R}$ in detrusor cells for purinergic contractions and $\mathrm{P} 2 \times 3 \mathrm{R}$ in urothelium, lamina propria cells and afferent nerves for sensory transmission. Using a selective $\mathrm{P} 2 \times 3$ receptor antagonist we assessed the role of urothelial $\mathrm{P} 2 \times 3 \mathrm{R}$ in the contractile responsiveness of intact or urothelium free bladders.

Methods: Female Sprague-Dawley rats were intravesically infused with saline or protamine (PR; $10 \mathrm{mg} / \mathrm{ml}$ for $30 \mathrm{~min}$ ). Open cystometry with saline followed by infusion of AF-353 (P2 $\times 3$ antagonist; $10 \mathrm{uM}$ ) was performed in urethane anesthetized rats. Bladder pressure during voiding (BPP), inter-contractile interval (ICI) and bladder compliance (BC) were calculated. Bladder wash was collected for ATP determination (luciferase). Electrical field stimulations (EFS; 100V/ $1 \mathrm{~ms} / 32 \mathrm{~Hz} / 10 \mathrm{~s}$ every $3 \mathrm{~min}$ ) were applied in longitudinal strips during sequential application of AF-353, abMeATP (10 UM) and atropine ( $\mathrm{uM})$. Contractile response to $\mathrm{KCl}(140 \mathrm{mM})$ was used for normalization. Timing and amplitude of purinergic contractions were calculated in response to abMeATP in different AF-353 concentrations. Histology was done with H\&E and IHC against P2 $\times 3$ (Abcam, ab90905; 1:2000). Data were analyzed with ANOVA or t-tests with $\mathrm{p}<0.05$ statistically significant.

Results: P2 $\times 3$ Rs are expressed in urothelial and lamina propria cells, with PR treatment removing most urothelial cells. Intravesical AF-353 (10uM) reduced BPP (25.5\%), and increased both ICI (46.0\%) and BC (100\%) in control but not in PR rats. Inhibition of $\mathrm{P} 2 \times 3 \mathrm{R}$ during $C M G$ produced a 4.6 fold increase in ATP from controls, without affecting high values in $\mathrm{PR}$ animals $(310+/-66$ vs $432+/-97 \mathrm{pmol} / \mathrm{void}$, saline vs AF353). $\mathrm{KCl}$ contractions were not affected by $\mathrm{P} 2 \times 3$ inhibition or urothelium removal. Inhibition of $\mathrm{P} 2 \times 3 \mathrm{R}$ prevents desensitization to abMeATP in intact but not PR strips during EFS. AF353 (10nM or 10uM) increased the response time to abMeATP (30\%) independently of urothelial integrity. Desensitization of purinergic contractions was prevented when $\mathrm{P} 2 \times 3 \mathrm{R}$ were blocked in urothelial cells.

Conclusions: $\mathrm{P} 2 \times 3 \mathrm{R}$ in urothelium and lamina propria may play a role in regulating the intravesical release of ATP as well as preventing $\mathrm{P} 2 \times 1 \mathrm{R}$ desensitization. Understanding the role of P2 $\times 3 \mathrm{R}$ in modulating contractile and sensory mechanisms would help to design more effective therapies for bladder dysfunction.

Support: Houston Methodist Hospital Foundation \& Brown Foundation

Clinical Relevance: LUTS/Voiding Dysfunction

\section{MODERATED POSTERS}

\section{Poster \#M1}

VOIDING SYMPTOMS AND TREATMENTS AFTER ROBOTIC ASSISTED PROLAPSE SURGERY (RAPS) 
Michael Ehlert, MD, Jamie Bartley, DO,

Kim Killinger, RN, MSN, Jason Gilleran, MD,

Melissa Fischer, $M D$

William Beaumont Hospital, Royal Oak, Michigan

(Presented by: Michael Ehlert, MD)

Introduction and Objectives: Voiding symptoms after surgery for pelvic organ prolapse (POP) are common and include denovo stress urinary incontinence (SUI), urinary urgency and frequency. We define the incidence and treatment of voiding symptoms after robotic prolapse repair.

Materials and Methods: Consecutive RAPS patients from 2007 to 2012 were identified. Those with post-operative voiding symptoms of stress incontinence or urinary urgency were further characterized, as were subsequent treatments.

Results: A total of 100 patients underwent attempted RAPS. Of these, 34 presented with 37 post-op voiding complaints. Mean age was $59( \pm 11)$. Previous surgeries included prolapse repair $(n=8)$, hysterectomy $(n=19)$, procedure for stress incontinence $(n=11)$. The majority presented with initial grade 3 POP (71\%) and multiple compartments involved (65\%). RAPS included 30 sacrocolpopexies and 4 sacrohysteropexies. Concomitant procedures included hysterectomy $(n=9)$, midurethral sling, $(n=11)$, primary posterior repair $(n=2)$, retropubic urethrolysis (1) and one urethral suspension. With an average follow-up of 8.2 months (median 5), 11 patients presented with de-novo stress incontinence and 3 with recurrent SUI after a mid-urethral sling (MUS). 2 patients had worse SUI post-op -1 declined sling pre-operatively, the other had an aborted sling due to a vaginal inclusion cyst. Management for SUI included mid-urethral sling $(n=6)$ at a median time of 48 days (range 35-2025), urethral bulking agents $(n=2)$ and observation $(n=8) .13$ patients complained of de-novo urinary urgency, and 8 with worsening urgency symptoms. Of these, 6 and 3 respectively had undergone sling at the time of sacrocolpopexy. Urgency was treated with medication in 1421 , sacral neuromodulation (1), and repeat transvaginal urethrolysis with Martius flap (1).

Conclusion: Voiding complaints are common after complex RAPS. A minority require additional procedures, while most can be managed non-operatively. Funding by MPURE philanthropic gift.

Clinical Relevance: Pelvic Organ Prolapse

\section{Poster \#M2}

\section{LASERVAPORIZATION OF THE PROSTATE WITH THE 180-W XPS-GREENLIGHT LASER IN PATIENTS WITH LARGE PROSTATES}

Daniel Lee, $\mathrm{MD}$, Malte Rieken, $\mathrm{MD}^{1}$, Fujun Zhao, $\mathrm{MD}^{1}$, Heike Pueschel, $\mathbf{R N}^{2}$, Bilal Chughtai, $\mathbf{M D}^{1}$, Steven Kaplan, $\mathbf{M D}^{1}$, Richard Lee, $\mathrm{MD}^{1}$, Alexander Bachmann, $\mathrm{MD}^{2}$ and Alex Te, $M D^{1}$

${ }^{1}$ Cornell, NY NY; ${ }^{2}$ University Hospital Basel

(Presented by: Daniel Lee, MD)

Introduction and Objectives: Photovaporization of the prostate (PVP) with the Greenlight ${ }^{\mathrm{TM}}$ laser is an established treatment option for the treatment of benign prostate enlargement. Despite the widespread use of the technique in patients, data on outcomes for patients with large prostates are limited. We aim to compare intermediate-term results of patients with prostate volumes greater vs. smaller than $100 \mathrm{ml}$.

Methods: A series of 362 patients who underwent PVP with the 180W XPS Greenlight ${ }^{\mathrm{TM}}$ laser (American Medical Systems, Inc.,
Minnetonka, MN) from July 2010 to June 2013 at two institutions was retrospectively studied. Perioperative parameters as well as postoperative functional results and complications were compared for those with prostate volumes $\geq 100 \mathrm{ml}$ vs. $<100 \mathrm{ml}$.

Results: Of 362 patients, 115 patients (31.8\%) had a transrectal measured prostate volume of $\geq 100 \mathrm{ml}$. Patients with a prostate volume of $\geq 100 \mathrm{ml}$ were older (75.3 vs. 71.0 years; $p=0.005$ ) and had a higher rate of preoperative retention $(53.0 \%$ vs. $29.6 \%$; $<$ 0.001) compared to patients with smaller prostate volumes. Median operating time (90.0 vs. $55.0 \mathrm{~min}$; $\mathrm{p}<0.001)$, lasing time (70.0 vs. 30.0 min; $p<0.001)$, applied energy (538 vs. $229 \mathrm{~kJ} ; \mathrm{p}<0.001$ ), and number of fibers used (2.0 vs. 1.0; $\mathrm{p}<0.001$ ) was significantly higher in patients with large prostates. Postoperatively, micturition symptoms (IPSS, OoL) and voiding parameters (Omax, postvoid residual volume) improved in both groups of patients, with no significant differences noted in the relative improvements. Over a followup of 2 years, the rates of postoperative urinary tract infection (3.5\% vs. $4.9 \% ; p=0.78)$, postoperative retention $(7.0 \%$ vs. $4.5 \% ; p=0.32$ ), urethral stricture (none vs. $1.2 \%, p=0.55$ ), and reoperation ( $1.7 \%$ vs. $1.6 \%$; $=1.00)$ did not differ between the two groups.

Conclusion: PVP with the 180W XPS Greenlight ${ }^{\mathrm{TM}}$ laser is a safe and effective minimal-invasive treatment option for patients with large prostate volumes. Although operative times and laser energy requirements are higher in patients with larger prostates, the rates of complications are not equivalent.

Clinical Relevance: BPH

\begin{tabular}{|c|c|c|c|}
\hline Weriobe & Wolumes $160 \mathrm{~cm}$ ? & Wolanos $\geq 100 \mathrm{~cm}$ ? & p-valiog \\
\hline N(06) & $247(08.746)$ & $115(31.5 \%)$ & \\
\hline Modian abs yours & 71.0 & 75.3 & $\infty .01$ \\
\hline Preoperitive arinary retention, $N(\omega)$ & $73(29.6 \%)$ & $61(53 \%)$ & $\$ .01$ \\
\hline Modian IFSS mpors, (QQR) & $17(13-24)$ & $21(16-24)$ & $\infty .01$ \\
\hline $\begin{array}{l}\text { Modian preoperidive } \mathrm{Qmax}, \mathrm{mL} / \mathrm{k} \\
\text { (COR) }\end{array}$ & $8.9(5.9-11.9)$ & $6.4(4.9-9.8)$ & $\infty .01$ \\
\hline Modian preoperifive FVR, mL(OQR) & $100(60-250)$ & $200(85-350)$ & 0,13 \\
\hline \multicolumn{4}{|l|}{ Operative } \\
\hline Modian opontíg, room time, min & 55 & 90 & 4.01 \\
\hline Modian hring time & 30 & 70 & $\infty .01$ \\
\hline 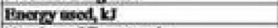 & 229 & 5388 & $\infty .01$ \\
\hline Nambar of fibers uned & 1.0 & 20 & $\infty .01$ \\
\hline \multicolumn{4}{|l|}{ Postopentive } \\
\hline 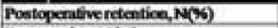 & $11(4.595)$ & $8(7,05)$ & 0.32 \\
\hline Reoperibion, $N(\mathbf{S})$ & $4(1.6 \%)$ & $2(1.7 \%)$ & 1.0 \\
\hline Postapentivitu,N( & $12(4.9 \%)$ & $4(0.5 \%)$ & 0.78 \\
\hline Postepenathemedhnd strkctare, $N(N)$ & $3(1.76)$ & 0 & 0.55 \\
\hline 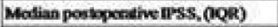 & $7(4-12)$ & $105(6-18)$ & $\infty .01$ \\
\hline 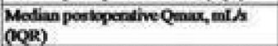 & $11.8(6.5-18.7)$ & $11.2(7.4-17.8)$ & 0,40 \\
\hline Motian poskepentive PVR, mL.(GQR) & $30(0-85)$ & $40(13-119)$ & 0.20 \\
\hline \multicolumn{4}{|l|}{ 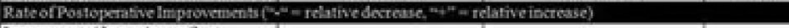 } \\
\hline \multicolumn{4}{|l|}{ 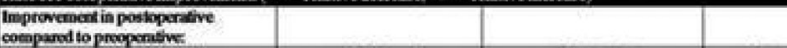 } \\
\hline ESS, Modian (QD) & $-10(15-5)$ & $9(16-4)$ & 0.71 \\
\hline Onax, Motinn (OOR) & $\cdot 4.4(0-5=95)$ & $.53(0.2+11,1)$ & 0.86 \\
\hline Pvir, Median (QQPE) & $74(250-9.0)$ & $146.5(284.5-27.5)$ & 0.28 \\
\hline
\end{tabular}

\section{Poster \#M3}

\section{EVOLUTIONARY STAGES IN URETHRAL STRICTURES}

Rajveer Purohit, MD, $\mathrm{MPH}^{1,2,3,4}$, Ricardo Georges, $\mathrm{BS}^{5}$, Matthew Benedon, BA ${ }^{6}$, Gabriel Mekel, MD $^{6}$ and Jerry Blaivas, $\mathbf{M D}^{2,3,4}$ ${ }^{1}$ Columbia University Medical Center-New York, NY; ${ }^{2}$ Weill Medical College of Cornell University-New York, NY; ${ }^{3}$ SUNY Downstate Medical Center-Brooklyn, NY; ${ }^{4}$ Institute of Bladder and Prostate Research-New York, NY; ${ }^{5}$ Institute of Bladder and Prostate Research; ${ }^{6}$ Institute of Bladder and Prostate ResearchNew York, NY

(Presented by: Ricardo Georges, BS)

Introduction and Objectives: Currently, there is minimal data evaluating progression of urethral strictures (US) from early to 
advanced stages. We examined data on male patients presenting with incidental early stage US and tracked their stricture progression on subsequent cystoscopies using a validated stricture staging system.

Methods: This is a retrospective analysis of 25 men with incidental findings of early stage US on cystoscopy (CS) between 1999 and 2013 for urological conditions other than US and who had multiple CS as follow-up for their primary condition. Diagnoses of urethral strictures were made at the time of cystoscopy according to a validated staging system: Stage 0 -no stricture; Stage 1-wide caliber stricture; Stage 2requires gentle dilation with a flexible cystoscope; Stage 3cannot be dilated; Stage 4-no visible lumen. Our study focuses only on patients with a Stage 1 stricture. No intervention was undertaken to treat the US in any of our patients.

Results: 33 patients were evaluated, 7 were excluded due to lack of follow up cystoscopies for a total of 25 men aged 44 to 88 (mean and median: 66) at the time of diagnosis. Mean number of cystoscopies (CS) were 9 (range: 2-46; median: 5); 12 for bladder cancer follow-up, 5 for suspected US, 4 for prostatic obstruction, and 1 each for bladder neck obstruction, neurogenic bladder, acontractile bladder, sphincteric incontinence, urethral diverticulum, urethrocutaneous fistula, and recurrent urinary tract infection. 5 patients had more than one reason for initial CS. Mean time between CS was $\sim 13$ months (median: 8) and mean time across all CS was $\sim 20$ months (median: 16.6). $24 \%(6 / 25)$ of patients demonstrated stricture progression during the follow-up. Of those that progressed, 33\% (2/6) advanced to stage 2, while $67 \%(4 / 6)$ progressed to stage 3 US. The mean time between stricture diagnosis to progression was $\sim 29$ months (median: 16). 24\% (6/25) of men demonstrated a regression of their US to stage 0 . The mean time between stricture diagnosis and regression was 12 months (mean: 12; median: 9). 52\% (13/25) of patients exhibited no change in US. Conclusion: Progression of US is uncertain and requires close follow-up for a better understanding of outcomes over time. Notably, $24 \%$ of patients both progressed and regressed in US severity. The staging system provided an easy lexicon for describing findings and documenting US evolution.

Clinical Relevance: LUTS/Voiding Dysfunction

\section{Poster \#M4}

\section{FESOTERODINE IN THE MANAGEMENT OF MEN WITH REFRACTORY OVERACTIVE BLADDER SYMPTOMS AFTER SURGERY FOR BLADDER OUTLET OBSTRUCTION}

Lily Wang, MD, PhD, Bilal Chughtai, MD, Asha Jamzadeh BA, Richard Lee, MD, MBA, Steven Kaplan, MD, Alexis Te, MD James Buchanan Brady Department of Urology, Weill Cornell Medical College of Cornell University, New York, NY

(Presented by: Lily Wang, MD, PhD)

Introduction and Objectives: We assessed the efficacy of fesoterodine in patients with refractory $O A B$ after treatment for benign prostatic hyperplasia (BPH).

Materials and Methods: This was a single center pilot study conducted in patients with overactive bladder (OAB) secondary to bladder outlet obstruction (BOO), refractory to anticholinergic medication and persistent for greater than 3 months after surgical intervention to relieve obstruction, with an International Prostate Symptom Score (IPSS) $>12$. Eighteen patients were enrolled in the study. Follow-up was performed at 2, 3, and 7 months. The primary endpoint was change in IPSS score. Secondary endpoints were maximum flow rate (Omax) and post-void residual (PVR).
Results: 18 patients were enrolled in the study, mean age was 72.1 (range $53-91$ ). Of the 18 patients 6 had significant side effects and did not complete the study. Patients receiving fesoterodine demonstrated significantly improved IPSS scores compared to baseline. (18.9 vs. $12.1 \mathrm{p}<0.05)$ In addition, the IPSS irritative sub-score improved from 6.2 to $2(p=0.023)$ at 7 months. Nocturia episodes decreased from 3 to $2.4(p=0.021)$. There was no significant change in IPSS obstructive sub-score, quality of life, PVR, or Omax. Of the 18 patients, 6 had significant side effects and did not complete the study. Three (17\%) patients had constipation, 1 (6\%) with vision change, and 2 (11\%) with dry mouth. Of the 11 patients who completed the study, 8 patients continued fesoterodine on a long term basis (greater than 15 months).

Conclusion: Fesoterodine was safe in patients with refractory irritative lower urinary tracts symptoms after surgical treatment of BPH. There were improvements in IPSS and nocturia. Larger trials are needed to help characterize the utility of fesoterodine in the treatment of OAB secondary to $\mathrm{BPH}$.

Funding was provided in part by Pfizer

Clinical Relevance: $\mathrm{BPH}$

\section{Poster \#M5}

THE FATE OF TRANSITIONAL UROLOGY PATIENTS REFERRED TO A TERTIARY CARE CENTER

Robert Chan, $\mathrm{MD}^{1}$, Saneal Rajanahally, $\mathrm{BS}^{2}$, Timothy Boone, $\mathrm{MD}, \mathrm{PhD}^{3}$ and Rose Khavari, $\mathrm{MD}^{3}$ ${ }^{1}$ Department of Urology, Houston Methodist Hospital, Houston, TX; ${ }^{2}$ Baylor College of Medicine, Houston, TX; ${ }^{3}$ Houston Methodist Hospital, Houston, TX

(Presented by: Robert Chan, MD)

Introduction: The transitional care clinic at the Center for Restorative Pelvic Medicine (CRPM) at Houston Methodist Hospital was established in 2010 to aid the transition of children with neurogenic bladder or genitourinary congenital anomalies to an adult urological team dedicated to their long term care. The aim of this study was to assess changes in management after transition to CRPM.

Methods: We performed a retrospective study of patients with neurogenic bladder or genitourinary congenital abnormalities referred to our CRPM in the age range of 16-26 years between 2010 and 2013. Data analyzed included patient characteristics cause of neurogenic bladder, method of bladder management, recurrent UTI, stones, kidney function, upper tract studies, video urodynamics, and change in management after transitioning care to CRPM.

Results: 21 patients were found with an average age of 21.9 $+/-2.6$ years (16-26). The etiology of their neurogenic bladder was spina bifida $11 / 24$, spinal cord injury $2 / 24$, transverse myelitis $2 / 24$, or other $9 / 24$. For management, we established a plan of care based on upper tract studies and video urodynamics to determine renal and bladder functionality. 18 of 21 patients underwent urodynamics and 12 of 21 had an upper tract study. Management after transitioning to CPRM was bladder augmentation or urinary diversion in $7 / 24$ patients, intravesical botox injections in 5/24 patients, and cystolitholapaxy or cystolithotomy in $2 / 24$ patients. For conservative measures, $2 / 24$ patients were started on anticholinergics and $1 / 24$ was started on CIC. Followup was $8.9+/-12.1$ months (050.2 months)

Conclusions: There is an immense need for transitional care of patients with neurogenic bladder or GU congenital abnormalities as they grow into adulthood. Nearly $71 \%$ of patients had a 
change in their bladder management with $38 \%$ undergoing a major surgery with bladder augmentation or cystectomy with diversion after presenting to the CRPM. This study emphasizes the necessity of a dedicated urologic team who has access to a comprehensive medical team to be involved in the care of these complex patients since their urological care and needs may vary significantly from their childhood.

Clinical Relevance: Reconstruction

\section{Poster \#M6}

\section{BARRIERS TO CARE IN PATIENTS WITH MULTIPLE SCLEROSIS}

Margarita Aponte, $\mathrm{MD}^{1}$, Areeba Sadiq $\mathrm{MS}^{1}$, Puspa Utomo ${ }^{2}$, Joseph Herbert, $M^{2}$, Nirit Rosenblum, $M^{1}$, Victor Nitti, $M^{1}$ and Benjamin Brucker, $\mathrm{MD}^{1}$

${ }^{1}$ Department of Urology, New York University Langone Medical Center, New York, NY; ${ }^{2}$ Department of Neurology, New York

University Langone Medical Center, New York, NY

(Presented by: Margarita Aponte, MD)

Introduction and Objectives: Up to $80 \%$ of multiple sclerosis (MS) patients experience urinary symptoms; however urologic evaluation and treatment are under-utilized in this population. The Actionable Bladder Symptom and Screening Tool (ABSST) is used to identify urinary symptoms in patients with MS and may select patients in need of urologic referral. The objective of this study is to identify barriers MS patients experience in seeking urologic care and its relationship to ABSST score.

Methods: This was a prospective observational study. 100 patients with MS, not currently seeing a urologist, were enrolled from an MS center with a urologist on site. Patients completed demographic information, a short form of the ABSST and questions to assess barriers to care. An ABSST score $>3$ met criteria for urologic referral. Two-month follow up calls assessed whether patients had seen a urologist. X2 tests were used to compare categorical variables.

Results: Of the 100 patients, there were 79 women and 21 men, mean age was 44.5 years and average time since MS diagnosis was 10.4 years. $40 \%$ of patients wanted to see a urologist and $33 \%$ had seen a urologist in the past. Most frequent reasons for seeking prior care were incontinence (46\%) and recurrent UTIs (24\%). Overall, the most common barriers to seeking prior care included "Doctor never referred"(18\%), "Doctor never asked"(15\%), "Had enough problems to deal with"(15\%), "Thought no treatments were available"(12.5\%) and "Felt embarrassed"(10\%). 40\% of men stated "Doctor never referred or asked"compared to $10 \%$ of women ( $p=0.002)$. 27 patients had an ABSST Score $>3$ and were more interested in seeing a urologist compared to those scoring $<3$ ( $91 \%$ vs. $40 \%$; $p=.000$ ). Patients with an ABSST $>3$ cited limitations associated with insurance, cost, transportation, or inaccessibility more often than those with an ABSST $<3$ (9\% vs. 3\%, $p=0.009$ ). After 2 months, 49 patients were reached for follow up. Despite persistent or worsening urinary symptoms in some patients, only 1 participant followed up with a urologist.

Conclusion: The ABSST is a valuable tool to identify MS patients with urinary symptoms willing to seek urological evaluation. Identification of this need alone unfortunately did not result in a significant increase in evaluation, despite ongoing symptoms. This underscores that other barriers, beyond awareness such as communication, costs and logistics, play a significant role in patients seeking urologic care.

Clinical Relevance: Neurogenic Bladder

\section{Poster \#M7}

SUPRAPUBIC TUBE PLACEMENT UNDER LAPAROSCOPIC GUIDANCE: A NOVEL TECHNIOUE

James Kearns, $\mathbf{M D}^{1}$, Gregory Bales, $\mathbf{M D}^{1}$

and Doreen Chung, $\mathrm{MD}^{2}$

${ }^{1}$ University of Chicago, Chicago, IL; ${ }^{2}$ Mount Sinai Hospital, Chicago, IL

(Presented by: James Kearns, MD)

Introduction: Suprapubic tube (SPT) placement is commonly performed in patients with neurogenic bladder. Known complications include bowel injury. Patients with high BMI, small bladder capacity and/or open bladder outlet limiting filling, and prior surgery remain a challenge for SPT placement. The use of laparoscopy to assist with visualization when inserting a SPT in these complicated patients may improve safety without the need for open surgery. The aim of this pilot study was to assess the safety and feasibility of SPT placement under laparoscopic guidance.

Methods: Five patients with neurogenic bladder underwent SPT insertion with laparoscopy between July 2012 and September 2013 at the University of Chicago Medical Center and Mount Sinai Hospital. A single $5 \mathrm{~mm}$ laparoscopic camera is introduced after abdominal insufflation with the Veress needle. The patient is placed in Trendelenburg position and the SPT is placed under direct vision.

Results: All patients included in the study had had prior abdominal surgery. There were 2 males and 3 females. Mean age was 52 years (range 43-58) and mean BMI was 28 (range 19-38). 3 (60\%) patients on physical exam had a gaping urethral meatus which limited bladder filling. 4 patients had SPT placement alone while one had a combined procedure with a laparoscopic bowel resection. For the patients who underwent SPT placement alone, mean operative time was 29 minutes (range 20-33). No complications occurred and estimated blood loss was minimal.

Conclusions: In this pilot study, SPT insertion under laparoscopic guidance appeared to be a safe, efficient, and feasible procedure with few complications. Further studies with greater numbers of subjected are needed to reinforce these results.

Clinical Relevance: Neurogenic Bladder

\section{Poster \#M8}

\section{LOWER URINARY TRACT SYMPTOMS AND URODYNAMIC FINDINGS IN PATIENTS WITH TRANSVERSE MYELITIS}

Myong Kim, $\mathrm{MD}^{1}$, Seong Jin Jeong, $\mathrm{MD}, \mathrm{PhD}^{2}$, Sung Yong Cho, $\mathrm{MD}, \mathrm{PhD}^{3}$ and Seung-June Oh, $\mathrm{MD}, \mathrm{PhD}^{1}$ ${ }^{1}$ Department of Urology, Seoul National University Hospital, Seoul, Korea; ${ }^{2}$ Seoul National University Bundang Hospital, Seongnam, Korea; ${ }^{3}$ SMG-SNU Boramae Medical Center, Seoul, Korea

(Presented by: Myong Kim, MD)

Introduction and Objectives: Transverse myelitis (TM) is an inflammatory disorder that affects a restricted area of the spinal cord. It has been reported the lower urinary tract symptom (LUTS) in TM patients is diverse according to involved level and it is similar to those of spinal cord injury. It is still obscure if the LUTS in TM patients is gone after treatment or is left as sequelae. However, there has been little information in the literature regarding the symptom variability according to the elapsed time. We aimed to find the correlation of the LUTS 
and location of lesion and time latency to urodynamic study (UDS) in TM patients.

Methods: We retrospectively reviewed medical records, imaging studies and UDS results of TM patients between Oct 2004 and Jun 2013. We identified the LUTS pattern, location of lesion, elapsed time to UDS, and analyzed the correlations.

Results: We identified 132 whose mean age of 43.8 ( \pm 21.6, SD) years. 90 patients $(68.2 \%)$ were males. The location of main lesion was at cervical spine in 36 patients (27.2\%), at thoracic spine in 75 patients (55.7\%) and at lower than lumbar spine in 22 patients (17.1\%). Mean duration of UDS from initial diagnosis of TM was 26.9 ( \pm 45.2$)$ months. Among them, 63 patients (47.7\%) and 52 patients (39.8\%) had urgency and urgency urinary incontinence (UUI). However, 31 patients (23.9\%) had an emptying failure and needed an aid for emptying such as clean intermittent catheterization. UDS showed 84 patients (63.6\%) had detrusor overactivity (DO) in filling cystometry. On the other hand, 27 patients $(20.5 \%)$ had underactive detrusor activity and 24 patients (18.2\%) had acontractile detrusor. In multivariate analysis, male $(\beta=1.502$, $p=0.013)$ and latent time to UDS $(\beta=0.035, p=0.036)$ were independent predictive variables for DO in UDS. On the other hand, age $(\beta=-0.051, p=0.004)$ and female $(\beta=1.497, p$ $=0.028$ ) were predictive variables for acontractile detrusor activity at voiding cystometry.

Conclusion: LUTS in TM patients was extremely broad from UUI to retention. Our results demonstrate that longer elapsed time to UDS is correlated with DO. Male and chronic disease state are risk factors for UUI, while female and young age is risky for retention in TM patients

\section{Poster \#M10}

\section{PROPERLY DOCUMENTING COMORBIDITIES IN FEMALE PELVIC MEDICINE AND RECONSTRUCTIVE SURGERY PATIENTS IS ESSENTIAL IN THE ERA OF HEALTHCARE REFORM}

Bradley Gill, MD, MS, James Ulchaker, MD, Sandip Vasavada, MD

Cleveland Clinic, Cleveland, $\mathrm{OH}$

(Presented by: Bradley Gill, MD, MS)

Introduction and Objectives: In the era of healthcare reform, accurate documentation of case complexity is increasingly necessary. As reimbursement becomes more scrutinized, practice sustainability may very well depend upon documentation of and appropriate payment for managing patients with concomitant medical issues. The goal of this study was to determine the impact of documenting comorbidities during hospital admissions on reimbursement patterns.

Methods: A retrospective review of all 2011-2012 tertiary center admissions by 4 staff female pelvic medicine and reconstructive surgeons occurred. The Centers for Medicare and Medicaid Services Diagnosis-Related Group (DRG) for admissions were recorded. The federal base rate of reimbursement for each DRG was obtained. Related DRG codes and base rates for the presence of complication or comorbidity (CC: such as dementia, acute blood loss anemia, acid-base disorder, abnormal body mass index, electrolyte abnormality, atelectasis, malnutrition etc.) as well as major complication or comorbidity (MCC: such as end stage renal disease, stage 3-4 pressure ulcer, sepsis, shock, severe protein-calorie malnutrition, encephalopathy, etc.) were also noted. The average admission patterns of each staff surgeon were used to assess how reimbursement was impacted by CC and MCC documentation and coding.
Results: Across commonly used DRG, base rates were $\$ 10,416-$ $\$ 3,414$, CC rates $\$ 15,580-\$ 4,980$, and MCC rates $\$ 30,706-$ $\$ 6,898$. Compared to base rates, the presence of CC or MCC provided an additional $36 \%$ to $197 \%$ reimbursement, with $97 \%$ mean. A total of 11-38 and 18-59 admissions per surgeon occurred in 2011 and 2012, respectively, with 14\%-44\% and $23 \%-40 \%$ being CC or MCC coded. Thus, annual inpatient admissions averaged 29 per surgeon with $29 \%$ being CC or MCC coded. Base rates were $\$ 15,580-\$ 3,495$ with a $\$ 7,590$ average. The mean reimbursement was $\$ 8731$ for CC or MCC and $\$ 5245$ for none. Overall, this produced an additional \$31,374 average annual reimbursement per surgeon for managing documented co-morbidities.

Conclusion: Appropriately documenting and being reimbursed for managing comorbidities can result in substantial changes to payment for care. Even routinely managed and seemingly minor cormorbid conditions should be specifically documented, as they can impact the coding of a hospital admission.

Clinical Relevance: Female Urology-including Incontinence

\section{Poster \#M11}

\section{MID-URETHRAL SYNTHETIC SLING COMPLICATIONS AND THEIR RELATIONSHIP TO POSITION AND MOBILITY ON TRANSLABIAL ULTRASOUND}

Leah Nakamura, MD, Judy Choi, MD, Diana Kang, MD, Tamara Hartshorn, MD, Erin Mellano, MD, Z. Chad Baxter, MD, Ja-Hong Kim, MD, Shlomo Raz, MD

Univeristy of California Los Angeles, Los Angeles, CA

(Presented by: Leah Nakamura, MD)

Introduction and Objectives: Mesh complications have been increasing and patients present with variable complaints. This study aimed to determine if sling position and mobility on translabial ultrasound (US) correlated with urethral pain and recurrent $(>2)$ urinary tract infections (UTIs) and their resolution after mesh removal.

Methods: A retrospective review was performed on all women who underwent a translabial US for mesh between January 2009 and February 2013. Only patients who had a synthetic suburethral sling were included in the analysis. Demographics and variables were obtained from medical records. Distance of the sling to the urethral lumen, craniocaudal (CC) and dorsoventral (DV) distance from the pubic symphysis on rest and valsalva, and CC and DV distance from the bladder neck to the sling were determined from translabial US. Statistical analysis was performed using t-tests, Fisher's exact test, and chi square analysis.

Results: There were 413 patients who underwent a translabial US for mesh. Patients were excluded for mesh placed for prolapse, multiple slings, or poorly visualized mesh. This left 128 patients for analysis. There were 44 patients who presented with dysuria or urethral pain and 69 patients with recurrent UTIs. The sling was distal in 32 patients, mid-urethral in 65, and proximal in 31 by US. 102 patients underwent surgery for mesh removal (56 partial and 46 complete mesh removal). Of the patients who had follow up data, $67.9 \%$ had resolution of their dysuria/urethral pain and $69.4 \%$ resolution of their UTIs. Patients with urethral pain or dysuria were more likely to have had a prior revision $(22.7 \%$ vs. $7.4 \%, \mathrm{P}=0.02)$ and less CC mobility $(0.48$ vs. $0.69 \mathrm{~cm}, \mathrm{P}=0.007)$ of the sling. Distance to the urethral lumen came close to being a significant predictor (0.23 vs. $0.29 \mathrm{~cm}, \mathrm{P}=0.057)$ of dysuria/urethral pain. Patients with resolution of their urethral pain or dysuria after 
mesh removal had more distal or proximal slings (CC pubic symphysis distance 1.5 vs. $2.29 \mathrm{~cm}, \mathrm{P}=0.015$ and CC bladder neck distance 0.52 vs. $0.57 \mathrm{~cm}, \mathrm{P}=0.013)$. Baseline demographics, amount of mesh removed, type of sling, obstruction, exposure, and other ultrasound measurements were not significantly associated with recurrent UTIs or dysuria/urethral pain.

Conclusion: Sling position and mobility on translabial ultrasound is associated with both presentation of urethral pain or dysuria as well as resolution of these symptoms after mesh removal.

Clinical Relevance: Female Urology - including Incontinence

\section{Poster \#M12}

\section{COMPARISON OF TRANSVAGINAL AND TRANSABDOMINAL} BLADDER NECK CLOSURE WITH SUPRAPUBIC CATHETER PLACEMENT IN WOMEN

Heather Willis, MD, Devin K. Sanders, BS, Nicole A. Safiano, BS, Keith Lloyd, MD

University of Alabama at Birmingham, Birmingham, $A L$

(Presented by: Heather Willis, MD)

Introduction: Bladder neck closure (BNC) is a relatively uncommon procedure that is reserved for patients with severe urethral incompetence and may be offered as an alternative to more extensive procedures involving bowel reconstruction. Use of a suprapubic catheter (SPC) as an alternative method of bladder drainage eliminates the need for bowel reconstruction, intermittent catheterization, and stoma appliances. BNC can be performed by a transvaginal (TV) or transabdominal (TA) approach. Data comparing the outcomes of the 2 approaches is limited. Based on our experience, we hypothesized that TV BNC is equally effective as TA BNC and is associated with less morbidity. The purpose of the study is to compare the urethral continence rates, peri-operative outcomes, short-term complications, long-term complications, and need for re-operation between TV BNC and TA BNC in females at our institution.

Methods: A retrospective review of 64 patients who underwent BNC with SPC placement from May 1990 to February 2013 was performed. Baseline variables as well as urethral continence rates after the first BNC procedure, ultimate urethral continence rates, length of operation, change in hematocrit, need for blood transfusion, blood loss, length of hospitalization, short and long-term complications, renal function by laboratory and radiologic studies, and need for re-operation were compared between TV and TA BNC. Fisher's exact tests and Wilcoxon rank-sum tests were used to compare outcomes between the treatment groups.

Results: Thirty-five women underwent TV BNC and 29 underwent TA BNC. Urethral erosion due to chronic in-dwelling urethral catheters to manage a neurogenic bladder was the most common indication for BNC overall (83.25\%). There were no differences in baseline comorbidities, renal function, level of neurologic injury, concomitant procedures, or age at surgery between TV and TA BNC. Urethral continence rate after the first BNC was not significantly different between the 2 groups (TV $85.71 \%$, TA $81.48 \% ; p=0.74)$. The only outcomes that were significantly different were median length of operation (TV 70.5 minutes, TA 112.0 minutes; $p=0.0003$ ) and median length of hospital stay (TV 1.0 day, TA 4.0 days; $\mathrm{p}<0.0001$ ).

Conclusion: There was no significant difference between TV and TA BNC in achieving urethral continence. TV BNC offers the advantage of a shorter length of operation and hospital stay. No financial funding was provided for this study.

Clinical Relevance: Female Urology-including Incontinence

\section{Poster \#M13}

A COMPARISON OF OUTCOMES AFTER RETROPUBIC SLING, DOES VALSALVA VOIDING AFFECT SUCCESS RATE?

Blake Anderson, $\mathrm{MD}, \mathrm{BS}^{1}$, David Hatcher, $\mathrm{MD}^{1}$, Rena Malik, $\mathrm{MD}^{1}$, Juraj Letko, $\mathrm{MD}^{2}$ and Doreen Chung, $\mathrm{MD}^{3}$ ${ }^{1}$ University of Chicago Medicine, Department of Surgery, Section of Urology, Chicago, IL; ${ }^{2}$ Mount Sinai Hospital, Chicago, IL; ${ }^{3}$ Mount Sinai Hospital and University of Chicago Medicine, Department of Surgery, Section of Urology, Chicago, IL (Presented by: Blake Anderson, MD, BS)

Introduction: The mechanism of action of the mid-urethral sling is not well understood but may rely on higher bladder outlet resistance during increased intra-abdominal pressure. It is known that many women void with valsalva and that retropubic slings (RPS) may cause voiding dysfunction. The aim of this study was to assess outcomes after RPS in women with and without valsalva voiding.

Methods: 42 women who underwent retropubic sling for stress incontinence (SUI) from2011 to2013 were retrospectively reviewed. Baseline demographics, urodynamic findings, operative parameters, and post-operative course were collected. Standardized follow-up was at 1, 3, and 6 months. The primary outcome was subjective success rate (absence of SUI with physical activity, coughing, or sneezing). We also looked at Urogenital Distress Inventory (UDI-6) score, Incontinence Impact Questionnaire (IIO-7) score, post-void residual (PVR), pad use, and revision rate.

Results: Subjects included 18(43\%) valsalva voiders (VV) and 24 (57\%) non-valsalva voiders (NV). No differences were seen between VV and NV in mean age, PVR, pad use, and UDI-6 score. VV had less severe IIQ-7 at baseline compared to NV, $(8 \pm 5$ vs. $13 \pm 7$ vs., $\mathrm{p}=0.04)$ and lower abdominal leak point pressure (ALPP) (53 vs. $75, p=0.02$ ). $67 \%$ of VV and $61 \%$ of NV passed initial void trial ( $p=0.237$ ). $5.6 \%$ of $\mathrm{VV}$ and $16.7 \%$ of $\mathrm{NV}$ required clean intermittent catheterization (CIC) $(p=0.23)$. No patients required CIC beyond 4 weeks postop in any group. Revision rates were not significantly different between VV $(5.6 \%)$ and $\mathrm{NV}(12.5 \%)(p=0.33)$ In total, 4 patients required revisions for pain (1 NV), erosion (1 NV), voiding dysfunction (1 $\mathrm{NV}$ ), or sling migration (1 VV). Subjective success rates were similar with no significant differences at 1, 3, and 6 months (83\%, 86\%, and $100 \%$ for VV and $96 \%, 100 \%$, and $100 \%$ for NV). There was no difference in postoperative change in PVR, UDI-6 and pad use compared to baseline between groups. VV had less of a decline in IIO-7 in comparison to NV at one and three months. ( $-5 \pm 7$ vs. $-12 \pm 7, p=0.002$ and $-4 \pm 9$ vs. $-10 \pm 6$, $\mathrm{p}=0.06)$.

Conclusion: No differences were seen in success rates of RPS in VV and NV. Furthermore the groups were similar in terms of CIC requirements, revision rates and postoperative parameters. Even in women with valsalva voiding, RPS appear to be a safe and effective treatment for stress urinary incontinence with low post-operative voiding dysfunction.

Funding Sources: none

\section{Poster \#M14}

ONABOTULINUMTOXINA SIGNIFICANTLY IMPROVES HEALTH-RELATED OUALITY OF LIFE IN PATIENTS WITH OVERACTIVE BLADDER SYNDROME: A POOLED ANALYSIS OF 2 PHASE 3 PLACEBO-CONTROLLED TRIALS

David Sussman, $\mathrm{DO}^{1}$, Blair Egerdie, $\mathrm{MD}^{2}$, Jihao $\mathrm{Zhou}, \mathrm{PhD}^{3}$, Kristin Khalaf, PharmD ${ }^{3}$, Christopher Nardo, $\mathrm{PhD}^{3}$ and Peter Sand, $\mathrm{MD}^{4}$ 
${ }^{1}$ University of Medicine and Dentistry of New Jersey, Sewell, NJ; ${ }^{2}$ Urology Associates/Urologic Medical Research, Kitchener, ON, Canada; ${ }^{3}$ Allergan, Inc., Irvine, CA; ${ }^{4}$ University of Chicago,

Evanston, IL

(Presented by: Peter Sand, MD)

Introduction and Objectives: OnabotulinumtoxinA (onabotA) $100 \mathrm{U}$ has been shown to significantly reduce urinary incontinence (UI) and provide treatment benefit in patients (pts) with overactive bladder syndrome $(O A B)$ who were inadequately managed by anticholinergics in 2 phase 3 trials. Here we present the effect of onabotA on health-related quality of life (HROOL) indicators in a pre-planned pooled analysis of the phase 3 trials, which were identical in design.

Methods: $\mathrm{OAB}$ pts with $\geq 3$ urgency UI episodes over 3 days and $\geq 8$ micturitions/day were randomized 1:1 to 20 cystoscopic intradetrusor injections $(0.5 \mathrm{~mL} /$ injection) of onabotA, total dose $100 U(n=557)$ or placebo (pbo) $(n=548)$, sparing the trigone. All pts were inadequately managed by prior anticholinergics. Prespecified secondary HROOL endpoints included mean change from baseline (BL) at week 12 in Incontinence-Quality of Life (I-OOL) total summary score and Kings Health Questionnaire (KHO) role limitations (RL) and social limitations (SL) scores. Increases from baseline indicate improvement on the I-OOL and Short Form-12 version 2 (SF12v2), while decreases indicate improvement on the KHO. Change from baseline in I-OOL domain scores, all other KHO domain scores, and SF-12v2 physical and mental component scores (PCS, MCS) were also assessed. Minimally important differences (MIDs, which reflect clinical significance) were +10 points for I-OOL, -5 for KHO, +2 for SF-12v2 PCS, and $+2-3$ for SF-12v2 MCS. HROOL measures were also evaluated in pts who did/did not initiate clean intermittent catheterization (CIC) after treatment.

Results: Mean BL HROOL scores were $\mathrm{I}-\mathrm{OOL}=34.5$, KHO $\mathrm{RL}=63.3, \mathrm{KHO} \mathrm{SL}=43.5, \mathrm{SF}-12 \mathrm{v} 2 \mathrm{PCS}=43.1$, and $\mathrm{SF}-12 \mathrm{v} 2$ $M C S=43.2$. Improvements from BL in I-OOL total, KHO RL and SL scores were significantly greater with onabotA vs pbo and exceeded established MIDs (22.5 vs 6.6 for I-OOL; -25.4 vs -3.7 for KHO RL; -16.8 vs -2.5 for KHO SL; all $\mathrm{P}<0.001)$. Improvements in I-OOL domain and other KHO domain scores also exceeded MIDs. 44/557 pts in the onabotA group and 8/548 in the pbo group used CIC temporarily after treatment. Improvements in disease-specific HROOL measures were similar in pts using/not using CIC after treatment. Increases in SF-12v2 PCS and MCS were also significantly greater with onabotA than pbo $(\mathrm{P}=0.012$ and $\mathrm{P}<0.001$, respectively)

Conclusion: OnabotA 100U resulted in significant and clinically-meaningful improvements in HROOL, regardless of CIC use. Funded by Allergan, Inc.

\section{Poster \#M15}

TREATMENT WITH ONABOTULINUMTOXINA SIGNIFICANTLY REDUCES EPISODES OF URINARY INCONTINENCE AND IMPROVES OUALITY OF LIFE IN FEMALE PATIENTS WITH IDIOPATHIC OVERACTIVE BLADDER SYNDROME

Peter Sand, Matthew Parsons, $\mathrm{MD}^{1}$, Jihao Zhou, $\mathrm{MD}, \mathrm{PhD}^{2,3}$, Denise Globe, $\mathrm{PhD}^{2,4}$, Christopher Nardo, $\mathrm{PhD}^{2,4}$ and Vik Khullar, $\mathrm{MD}^{5}$

${ }^{1}$ Birmingham Women's Hospital NHS Foundation Trust, Birmingham, UK; ${ }^{2}$ Allergan, Inc; ${ }^{3}$ Bridgewater, $N J ;{ }^{4}$ Irvine, CA; ${ }^{5}$ Imperial College, London, UK

(Presented by: Peter Sand)
Introduction and Objectives: Overactive bladder (OAB) syndrome with urgency urinary incontinence (UUI) is disproportionately more common in women than men. Two large phase 3 trials evaluated the efficacy and safety of onabotulinumtoxinA (onabotA) $100 \mathrm{U}$ in OAB patients (pts) with urinary incontinence (UI) who were inadequately managed by anticholinergics (ACHs). Here we report a pre-planned analysis of the efficacy and safety of onabotA in the female subpopulation from the pooled phase 3 trials.

Methods: OAB pts $(\mathrm{N}=1105)$ with UUI or mixed incontinence with predominant UUI were randomized 1:1 to receive 20 cystoscopic intradetrusor injections $(0.5 \mathrm{ml} /$ injection) of onabotA 100U $(n=557)$ or placebo (pbo) $(n=548)$, sparing the trigone. Pts with a predominance of stress UI were excluded. All pts were inadequately managed by prior ACHs. Change from baseline (BL) at week (wk) 12 in UI episodes/day and proportion of patients reporting a positive response (condition 'greatly improved' or 'improved') on the treatment benefit scale (TBS) were evaluated in the pooled female pts $(n=970)$. Healthrelated quality of life (HROOL) outcomes were assessed using the Incontinence-Ouality of Life instrument and King's Health Ouestionnaire. Adverse events (AEs) were assessed.

Results: Mean UI episodes/day at BL in onabotA and pbo were 5.5 and 5.6, respectively, in female pts and 5.5 and 5.4 in the overall pooled population. At wk 12, significant reductions in UI episodes/day were seen with onabotA vs pbo in female pts $(-2.92$ vs $-0.90 ; \mathrm{P}<0.001)$ which were comparable to the overall population $(-2.80$ vs $-0.95 ; \mathrm{P}<0.001)$. A significantly higher proportion of onabotA pts vs pbo reported a positive response on the TBS in the female subpopulation (64.3 vs $28.4 \%$; $\mathrm{P}<0.001$ ), and the overall population ( 61.8 vs $28.0 \% ; \mathrm{P}<0.001$ ). Clinically meaningful improvements in pts' HROOL were seen with onabotA but not pbo in all domains except General Health, which were also statistically significant with onabotA vs pbo, in both the female pts and the overall population. AEs were localized to the bladder; urinary tract infection was the most common AE in female pts (27.4\% with onabotA vs $10.6 \%$ with pbo), and the overall population (25.5\% with onabotA vs $9.6 \%$ with pbo).

Conclusion: OnabotA 100U was well tolerated and demonstrated significant reductions in UI episodes and improvements in HROOL compared with pbo, in female pts with $O A B$ who were inadequately managed by ACH therapy.

Funding: Allergan., Inc.

Clinical Relevance: Female Urology-including Incontinence

\section{Poster \#M16}

URINARY RETENTION RATES AFTER INTRAVESICAL ONABOTULINUMTOXINA INJECTION FOR OVERACTIVE BLADDER IN CLINICAL PRACTICE AND PREDICTORS OF THIS OUTCOME

David Osborn, MD, Melissa Kaufman, MD, PHD, Stephen Mock, MD, Meghana Gowda, MD, Osa Okunbor, Xuechao Zhang, Nicklaus Rice, MD, Michael Guan, Roger Dmochowski, MD, W. Stuart Reynolds, MD Nashville, TN

(Presented by: David Osborn, MD)

Introduction: The purpose of this study was to find the rate of urinary retention in clinical practice after treatment with OnabotulinumtoxinA (BTN/A) for medication-refractory overactive bladder $(\mathrm{OAB})$ symptoms and determine factors that predict this outcome. 
Materials and Methods: This is a retrospective study of consecutive patients who underwent intravesical BTN/A injection for non-neurogenic, medication refractory $O A B$. Patients were analyzed with respect to their first and second BTN/A injections. The primary outcome measure was postoperative urinary retention. The associations between the various independent variables were assessed with multivariate logistic regression. Inclusion and exclusion criteria for the study were similar to those of the recent BTN/A clinical trials.

Results: Based on inclusion and exclusion criteria, 160 patients were eligible for analysis. Mean age was 64 years and $24 \%$ of the patients were men. Overall, $64 \%(n=102)$ of patients reported subjective improvement in their symptoms. The rate of urinary retention was $35 \%(n=56)$ and the mean length of daily intermittent catheterization was 16 weeks (1-40). For the first BTN/A treatment, multivariate analysis revealed that an elevated preoperative PVR (postvoid residual volume) (OR 1.27, 95\% CI 1.13-1.43, p < 0.001) and pre-operative bladder capacity (OR 1.05, 95\% CI 1.05-1.09, p 0.002) were associated with postoperative urinary retention. In patients with a preoperative PVR of $\geq 100 \mathrm{ml}, 94 \%(n=17)$ went into urinary retention. Eighty-seven (54\%) patients who had postoperative urinary retention went on to repeat treatment with BTN/A. For those patients who underwent a second BTN/A treatment, preoperative PVR (OR 1.19, 95\% CI 1.03-1.38, p 0.02), BTN/A units injected (OR 4.22, 95\% CI 1.15-15.49, p 0.03) and retention after the first BTN/A (OR 30.2, 95\% CI 5.18-175.92, p <0.001) were associated with increased rate of postoperative retention.

Conclusion: Despite what is reported in recent clinical trials, patients with an increased preoperative PVR should be counseled that they have a higher likelihood of requiring postoperative CIC. The retention rate of $35 \%$ is higher than that reported in recent clinical trials. The inclusion of patients with a preoperative PVR $\geq 100 \mathrm{ml}$ and the fact that clinicians in this study were not influenced by being part of a clinical trial and demonstrated a lower threshold to initiate CIC contributed to the high rate of urinary retention found in this study.

Clinical Relevance: LUTS/Voiding Dysfunction

\section{Poster \#M17 \\ THE EFFECT OF PREGNANCY ON INTERSTITIAL CYSTITIS/ BLADDER PAIN SYNDROME}

Peter O'Hare, III, MD, Dominique El-Khawand, MD, Darlene Morrissey DO, Kristene Whitmore, MD

Drexel University College of Medicine

(Presented by: Peter O'Hare, III, MD)

Objective: To describe the symptoms and course of Interstitial Cystitis/Bladder pain syndrome (IC/BPS) throughout pregnancy and with subsequent pregnancies.

Methods: In February 2011, a survey of pregnant or postpartum women in the US, diagnosed with IC/BPS was conducted. Respondents indicated if they received an IC/BPS diagnosis and described the severity of their disease prior to pregnancy. On a 5-point likert scale, respondents rated their urinary symptoms in each trimester, the postpartum period and for any prior pregnancies.

Results: 564 respondents confirmed IC/BPS diagnosis by a physician. Among 384 women who reported a pregnancy after their IC/BPS was diagnosed, their pre-gestational symptoms were rated mild (30\%), moderate (52\%) and severe (18\%). Compared to pre-pregnancy levels, pain was rated better in the first trimester, second trimester and third trimester in $39.3 \%$,
$51.8 \%$ and 44.2 , and worse in $25.1 \%, 28.8 \%$ and $39 \%$, respectively $(\mathrm{n}=303)$. Similarly, urinary frequency was rated better in $27 \%, 35.8 \%$ and $27.6 \%$, and worse in $39.5 \%, 42.8 \%$ and $56.3 \%$, while urinary urgency was rated as better in $39.3 \%$, $51.8 \%$ and $44.2 \%$ and worse in $25.1 \%, 28.8 \%$ and $39 \%$, in the first, second and third trimesters respectively. Patients' reported symptom change from baseline for pain, frequency and urgency positively correlated across all trimesters $(p=<0.001$, rho 0.38-0.93). Similarly, a positive correlation was found between the same symptoms across the first and second pregnancies $(p=<0.001$, rho 0.5-0.6). Breastfeeding maintained the subjective symptom change found during the gestation as evident by a positive correlation $(p=<0.05$, rho $0.16-0.36)$; symptoms were rated as better in $52 \%$, unchanged in $38 \%$ and worse in $10 \%$ compared to pre-gestation baseline. Pregnancy outcomes $(n=351)$ were reported as $81 \%$ live births, $3.4 \%$ miscarriages $0.3 \%$ still birth, and $1.1 \%$ termination. Two out of 5 terminations were performed due to severe IC/BPS symptoms. Seventy three percent of patients reported discontinuing their regular IC/BPS treatment during pregnancy and $77 \%$ reported no other alternatives offered by their physician. Post partum symptom level returned to baseline in $63 \%$ of patients with a mean of 27 weeks $( \pm 66)$.

Conclusion: Pregnancy may improve pain in more than half of patients with IC/BPS in the second trimester. The effect of pregnancy on IC/BPS symptoms appears to remain stable throughout the gestation, breastfeeding and a subsequent pregnancy.

Clinical Relevance: IC and Pelvic Pain-UTI / Inflammatory

\section{Poster \#M18}

\section{CLINICAL PREDICTORS OF BLADDER OUTLET OBSTRUCTION} ON URODYNAMICS

Joshua Cohn, $\mathrm{MD}^{1}$, Rena Malik, $\mathrm{MD}^{1}$, Gregory Bales, $\mathrm{MD}^{1}$ and Doreen Chung, $\mathrm{MD}^{2}$

${ }^{1}$ University of Chicago, Chicago, IL; ${ }^{2}$ Sinai Hospital and the University of Chicago, Chicago, IL

(Presented by: Joshua Cohn, MD)

Introduction: Initial work-up of a patient presenting with lower urinary tract symptoms (LUTS) consists of detailed history, exam and post-void residual (PVR). We aimed to assess the ability of these factors to predict for obstruction on urodynamics (UDS).

Methods: A single center UDS database was queried for all patients undergoing UDS from 2010-2013. In men, bladder outlet obstruction (BOO) was defined as BOO index $>40$. In females BOO was defined as maximum flow rate (Omax) $<12 \mathrm{~mL} / \mathrm{sec}$ with detrusor pressure at Omax $>20 \mathrm{cmH} 2 \mathrm{O}$ on pressure-flow study. Logistic regression was performed to assess predictors of obstruction in all patients and by gender with subset analysis in males with clinical diagnosis of benign prostatic hyperplasia (BPH).

Results: The study population consisted of 450 patients patients of whom 304 (68\%) were women and 146 (32\%) men. 34 men (8\%) had a clinical diagnosis of BPH. On univariate analysis of all patients, age (OR 1.05, 1.01-1.07), presence of voiding LUTS (OR 2.56, 1.44-4.53), post-void residual of 50$100 \%$ of capacity (OR 5.58, 2.13-14.61) and inability to void (OR 7.11, 2.83-17.87) predicted for increased odds of BOO on UDS whereas female gender (OR 0.05, 0.03-0.09), body-mass index (OR 0.93, 0.89-0.97) and presence of storage LUTS (OR 0.39, 0.24-0.63) were associated with decreased odds. On multivariate analysis, age $(p=0.01)$, female gender $(p<0.01)$ and 
presence of voiding LUTS $(p=0.05)$ remained significant predictors. On multivariate analysis in only female patients adjusting for age, storage/voiding LUTS and PVR, only presence of voiding LUTS (OR 3.32, 1.08-10.25) remained a significant predictor of obstruction. In the same analysis in men, age (OR $1.03,1.00-1.06)$ was the only significant predictor of BOO on UDS. On univariate analysis of men with clinical diagnosis BPH, neither voiding LUTS nor PVR were significant predictors of BOO; presence of storage LUTS (OR 0.16, 0.02-0.99) was the only significant predictor.

Conclusions: In women, voiding LUTS suggest increased likelihood of a significant obstructive component on UDS, which should be considered in initial management. In men with clinical diagnosis of BPH, classic symptoms such as voiding LUTS and PVR may not reliably predict for BOO on UDS, whereas presence of storage LUTS may suggest decreased likelihood of BOO. Although commonly not performed prior to $\mathrm{BPH}$ surgery, increased consideration should be given to UDS prior to definitive intervention in men.

Funding: none

Clinical Relevance: BPH

\section{Poster \#M20}

\section{CHRONIC LOWER URINARY TRACT SYMPTOMS IN YOUNG MEN WITHOUT SYMPTOMS OF CHRONIC PROSTATITIS: URODYNAMIC ANALYSES IN 308 MEN AGED 50 OR LESS}

Seong Jin Jeong ${ }^{1}$, Jin Woo Jeong, $\mathbf{M D}^{2}$, Sang Cheol Lee, $\mathbf{M D}^{2}$ and Sang Eun Lee, $\mathrm{MD}^{2}$

${ }^{1}$ Seoul National University Bundang Hospital, Seongnam;

${ }^{2}$ Department of Urology, Seoul National University Bundang Hospital, Seongnam, Korea

(Presented by: Seong Jin Jeong)

Introduction and Objectives: Chronic lower urinary tract symptoms (LUTS) among young men often yield diagnostic dilemmas. Previous studies only considered small populations and focused on men who had previously diagnosed with chronic prostatitis. We investigated the etiologies of LUTS and compared urodynamic characteristics between different diagnostic groups in young men with chronic LUTS.

Methods: We reviewed the medical records of 308 men aged between 18 and 50 who had undergone an urodynamic study for chronic LUTS ( $\geq 6$ months) without symptoms suggestive of chronic prostatitis.

Results: Mean age was 40.4 ( \pm 10.1 ) years and mean symptom duration was 38.8 ( \pm 49.2) months. Urodynamic evaluations demonstrated primary bladder neck dysfunction (PBND) in $26.0 \%$ of cases, dysfunctional voiding (DV) in $23.4 \%$, and detrusor underactivity (DU)/acontractile detrusor (AD) in $12.7 \%$ as voiding dysfunction (Table 1 ). As a single storage dysfunction, detrusor overactivity (DO) was found in $13.3 \%$, small cystometric capacity in $17.9 \%$, and reduced bladder sensation in 5.2\% (Table 1). Most of demographics and types of clinical symptoms were not different between different diagnostic groups. While $53.9 \%$ of voiding dysfunctions had concomitant storage dysfunctions, $69.6 \%$ of storage dysfunctions were found to have concomitant voiding dysfunctions. Men with DV or DU/AD exhibited lower maximum cystometric capacities than those with normal urodynamics. Low bladder compliance was most frequent in PBND (10.0\%, p =0.025). In storage dysfunctions, men with DO were shown to have higher detrusor pressure in voiding than those with the other storage dysfunctions $(p<0.01)$.
Conclusion: As a single specific dysfunction, PBND was most frequent, followed by DV and DU/AD. Storage dysfunctions were frequently associated with concomitant voiding dysfunctions. Urodynamic investigation in this population is helpful in making an accurate diagnosis and may guide adequate treatment as clinical symptoms are not useful in predicting a specific urodynamic etiology.

Clinical Relevance: LUTS / Voiding Dysfunction

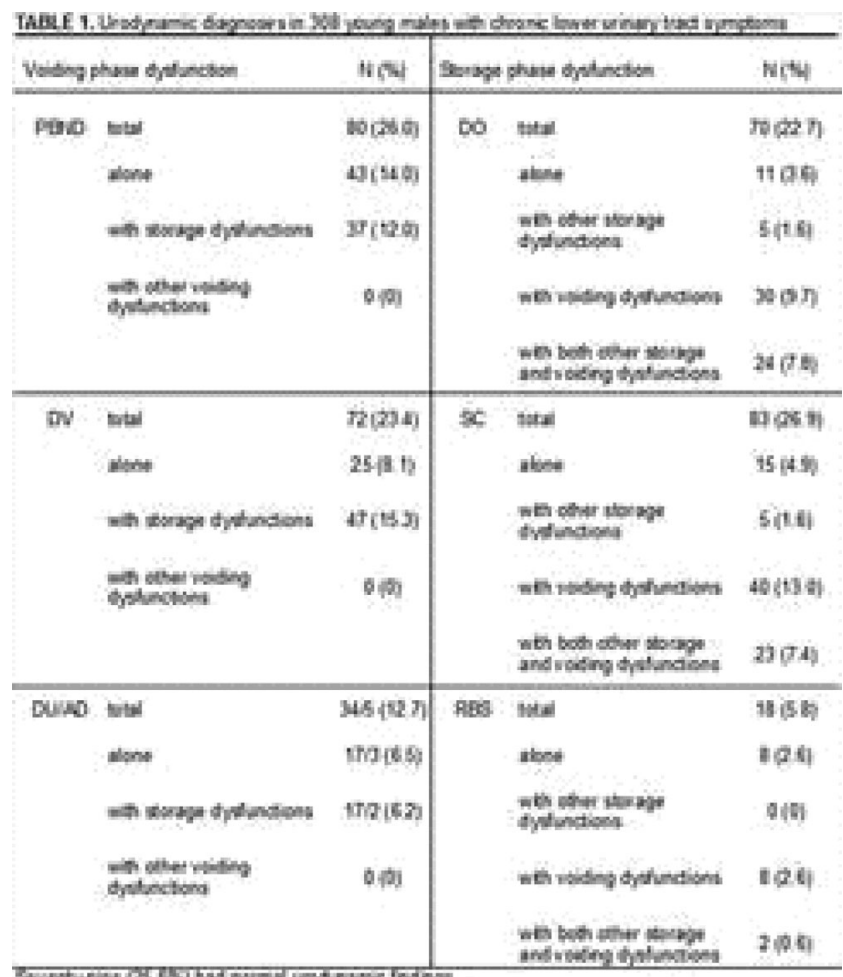

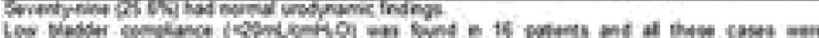

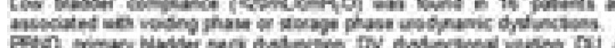

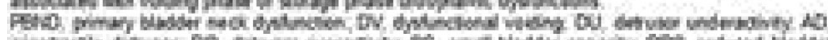

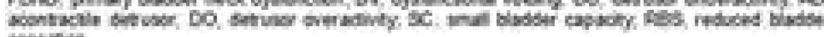
intiven

Poster \#M21

URODYNAMIC ASSESSMENT OF BLADDER AND URETHRAL FUNCTION AMONG MEN WITH RADICAL PROSTATECTOMY: A COMPARISON BETWEEN MEN WITH AND WITHOUT URINARY INCONTINENCE

Seong Jin Jeong ${ }^{1}$, Hahn-Ey Lee, $\mathrm{MD}^{2}$, Myung Kim, $\mathrm{MD}^{2}$, Sung Yong Cho, $\mathrm{MD}^{3}$ and Seung-June Oh, $\mathrm{MD}^{2}$

${ }^{1}$ Seoul National University Bundang Hospital, Seongnam; ${ }^{2}$ Seoul National University Hospital, Seoul; ${ }^{3}$ SMG-SNU Boramae Medical Center, Seoul

(Presented by: Seong Jin Jeong)

Introduction and Objectives: The changes of bladder and urethral functions after radical prostatectomy (RP) have not been extensively studied. Using a large scale database from SEOUL (SNU-Experts-Of-Urodynamics-Leading) Study Group in Korea, we compared bladder and urethral functions following RP between men with and without urinary incontinence.

Methods: Since July 2004, we have prospectively collected data on urodynamics in 303 patients with lower urinary tract symptoms (LUTS) following RP at three affiliated hospitals of SEOUL Study Group. After excluding 35 patients with neurogenic abnormality, pelvic irradiation after surgery, or a history 
of surgery on the lower urinary tract, 268 men were evaluated. We compared urodynamic findings following RP between men who had LUTS with urinary incontinence (post-prostatectomy incontinence (PPI) group: $\mathrm{n}=150$ ) and those had LUTS without urinary incontinence (non-PPI group: $n=118$ ).

Results: The mean age at surgery was $66.3( \pm 8.3)$ years and mean age at an urodynamic study was $68.2( \pm 6.8)$ years. By surgical approach, 128 (47.8\%) patients underwent open retropubic RP, 7 (2.6\%) laparoscopic RP, and 133 (49.6\%) robotassisted RP. Overall, reduced bladder compliance $(\leq 20 \mathrm{ml} /$ $\mathrm{cmH2O})$ was shown in $73(27.2 \%)$ patients and $84(31.3 \%)$ patients had idiopathic detrusor overactivity. Patients in PPI group were older $(p=0.001)$ at an urodynamic study and had lower maximum urethral closing pressure $(p<0.001)$ and shorter functional urethral length $(p=0.035)$ compared to those in non-PPI group. In addition, bladder capacity and detrusor pressure during voiding were significantly lower in PPI group. The percent of patients with detrusor underactivity was not different, while bladder outlet obstruction was more prevalent in non-PPI group (Table). Fourteen (9.3\%) patients of PPI group did not demonstrate urine leakage, while 40 (33.9\%) patients of non-PPI group showed urine leakage.

Conclusion: About one-quarter and one-third of patients with LUTS following RP had reduced bladder compliance and detrusor overactivity in the urodynamic assessment. Urinary incontinence following RP might be associated with both impairment of urethral function and lower bladder capacity during storage phase.

Clinical Relevance: Male Incontinence

\begin{tabular}{|c|c|c|c|}
\hline \multicolumn{4}{|c|}{ Toble. Results secording to the presence of urinary incontinenee } \\
\hline & PPI group & Noa,prt proup & provive \\
\hline Wumber of patients & 150 & 118 & \\
\hline Age at Ured, numics ot & $69.4=60$ & $657+7.4$ & 0.001 \\
\hline 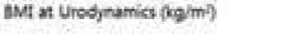 & $247+28$ & $259=116$ & 0.282 \\
\hline \multicolumn{4}{|l|}{ Free ureflowmetry } \\
\hline Mavimal flow rase (mi/hec) & 15.50 & $143+20$ & a.1s9. \\
\hline Pot-noid widuat utich imbi & 212 it th & $221+267$ & OMas \\
\hline \multicolumn{4}{|l|}{ Unethral pesckure profilometry } \\
\hline Maximum sloing pensure (omitio) & $602 \div 25.5$ & $7010=195$ & $\$ 0.001$ \\
\hline Funtiond profie ingth (mm) & $464+16.4$ & $511+214$ & o.as \\
\hline \multicolumn{4}{|l|}{ Filling opstometry } \\
\hline Fint desine so vold (m) & $2016+853$ & $221.4+81.4$ & a.037 \\
\hline Nermal detire te vad (mil) & $2769+126$ & $120:=1054$ & 0.001 \\
\hline strong deshe to void $(m i)$. & $341.9+1604$ & $344.6=1008$ & 6.001 \\
\hline Diduced bludder tentabon & $5(22 x)$ & $7(5)$ & $0.00 \%$ \\
\hline Compliance $\$ 20 \mathrm{~m} / \mathrm{am} H \mathrm{j}$ & $4(217 \mathrm{w})$ & $10(25,40)$ & assa \\
\hline Inoluntery detruser contraction & $49(327)$ & $35(29.7 \times)$ & 0.691 \\
\hline ALPp leak positive & 136 (907) & 40 (3) 9.4) & *0.001 \\
\hline Leak pressure iomityol & $1221+543$ & $147.8 \times 35.1$ & 0.001 \\
\hline \multicolumn{4}{|l|}{ Velding wpsometry } \\
\hline Pdergmax $(0=H ; O)$ & $247+147$ & $150+14.9$ & soucoi \\
\hline Cpening pressure (mmit,o) & $25.5 \times 112$ & $30.5,141$ & $-0,051$ \\
\hline Mavimal fow ous inlites & $127+8 s$ & $11.6 \times 75$ & o.ose \\
\hline Poat-wold tridual uhe inll & stit: 62 & $145+1154$ & o.sie \\
\hline 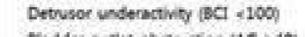 & $94 \cdot 62.74)$ & $75(636 \%)$ & Q.993 \\
\hline 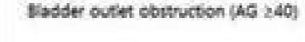 & $6.4000)$ & $15,127 \%$ & aons \\
\hline
\end{tabular}

\section{Poster \#M22}

OUTCOME OF SACRAL NEUROMODULATION (SNM) FOR REFRACTORY OVERACTIVE BLADDER (OAB) IN MEN WITH PRIOR HISTORY OF TRANSURETHRAL RESECTION OF PROSTATE (TURP)

Stephen Mock, MD, David J. Osborn, MD, Anne Scott, MD, Melissa Kaufman, MD, Roger R. Dmochowski, MD, W. Stuart Reynolds, MD

Vanderbilt University, Nashville, TN

(Presented by: Stephen Mock, MD)
Introduction and Objectives: Lower urinary tract symptoms (LUTS) are a common problem affecting men. TURP is an effective therapy if medical management fails. While $O A B$ symptoms is relieved by TURP as well in a majority, refractory cases exist that can have an adverse impact on quality of life. SNM has proven efficacy in refractory $\mathrm{OAB}$ and urinary urge incontinence (UUI) but there is scant data available in the post TURP setting. The aim of this study is to determine the outcome of SNM on post TURP refractory OAB and UUI.

Methods: A total of 490 patients underwent SNM between 2000 and 2012. One hundred and twenty six (26.4\%) were male. Within this, 38 (30.1\%) men had a prior history TURP. Urinary morbidity was recorded using the International prostate symptom score (IPSS) pre SNM and at last follow-up. Pre TURP IPSS was not available in a majority. Success was defined as improvement in IPSS of 6 or greater. Failure was defined as IPSS improvement less than 6 , worsening of IPSS, or explantation of device due to lack of efficacy.

Results: Twenty patients were eligible for analysis. Median age at SNM was 60.5 years old. Average number of TURPs was 1.7. Median time from last TURP to SNM was 40 months (range 8 to 160 months). Median follow-up after SNM was 29 months (range 2 to 106 months). Detrusor over-activity (DO) was demonstrable in 10 patients. No patient had bladder outlet obstruction. Median pre SNM IPSS was 26.5 (range 7 to 32). Median IPSS at last follow-up was 22 (range 3 to 35). Six of twenty (30\%) men had success from SNM. IPSS at last follow-up was lower for responders than non-responders (7 vs. 29, $\mathrm{p}<0.05)$ Univariate analysis for variables that may predict success was not significant for age, pre SNM IPSS, history of diabetes, history of spinal surgery, or presence of DO on UDS. Presence of neurological disease was significant for response to SNM $(75 \%$ vs. $19 \%, p=0.03)$ as was history of a psychiatric disease ( $55.6 \%$ vs. $10 \%, p=0.02)$

Conclusion: Refractory OAB post TURP is not uncommon. SNM yielded poor results in this setting and may be that this represent a fairly complex cohort; a majority had multiple interventions prior to initial consultation and there was a long protracted course from TURP to SNM. The presence of neurological or psychiatric disease was a predictor of success of SNM in post TURP patients.

Source of Funding: None

Clinical Relevance: LUTS / Voiding Dysfunction

\section{Poster \#M23}

POSTERIOR TIBIAL NERVE STIMULATION (PTNS) FOR TREATMENT OF NON-NEUROGENIC DETRUSOR OVERACTIVITY AND CHRONIC PELVIC PAIN

Riyad Almousa, MD, Mohammed Ali Aggamy, MD, Adel Aldayel, MD, Mohammed Gomha, MD

King Fahad Specialist Hospital Dammam

(Presented by: Riyad Almousa, MD)

Introduction \& Objectives: To assess the efficacy of Posterior Tibial Nerve Stimulation (PTNS) in treatment of lower urinary tract filling symptoms (urgency, frequency, urge incontinence) and chronic pelvic pain in patient with Non Neurogenic Detrusor Overactivity and/or chronic pelvic pain unresponsive to medical therapy.

Material \& Methods: It is a prospective single centre study. Between March 2010 -February 2012,30 patients with Detrusor overactivity (urgency, frequency with/without incontinence) and/or chronic pelvic pain unresponsive to medical therapy underwent PTNS therapy using Urgent PC kits weekly 
for a total of 12 sessions (30 minutes sessions each). Patients who were considered as success completed another twice/ month sessions for three months then once/month sessions for another 6 months (total of 12 months therapy). All patients had baseline investigations. Each patient filled a voiding diary, quality of life questionnaire and pain scale at the beginning of therapy, and after 12 weeks. The analyzed variables included: daytime and nighttime voiding frequency, voiding volume, number of and estimated amount of urine leak episodes and attacks of pelvic pain. These variables were recorded in a data capture forms using SPSS 14 program. Data were retrieved from standard voiding diary (Arabic form) as recommended by ICS guidelines. Our primary objective outcome was at least $50 \%$ reduction in one or more variables in addition to patient satisfaction to consider therapy as successful.

Results: All 30pts completed at least the initial 12 sessions. No one stopped therapy due to side effect or pain related to the treatment.10 males,20females(1:2). Age range 19yrs-80yrs (mean age 32). Mean voids per day decreased from11.3to7.1 (-37.1\%). After treatment,63\%rated themselves as much better or better in term of pelvic pain; more than $68 \%$ completely or somewhat satisfied. Nocturia episodes were significantly reduced post therapy (mean $=-0.70$ vs. -0.32 episodes/night; $\mathrm{P}=.05$ )There were greater reductions in maximum urgency attacks/day (mean $=-0.44$ vs. $-0.12 ; \mathrm{P}=.02$ )

Conclusion: This study demonstrates that (PTNS) could be considered as a safe and effective therapy in Non Neurogenic detrusor overactivity and/or chronic pelvic pain patients who failed medical treatment. Cost effectiveness and long term effects need to be evaluated with longer follow-up and higher number of patients to assess the feasibility of considering PTNS therapy as an alternative treatment to medical therapy.

Clinical Relevance: Neuromodulation

\section{Poster \#M24}

\section{INTRAVESICAL ONABOTULINUMTOXIN-A INJECTIONS: CLINICAL IMPLICATIONS OF INJECTION PARAMETERS}

Sara Lenherr, MD, Duncan Morhardt, MD, PhD, Heather Crossley MS, Anne Cameron, MD, Ann Oldendorf, MD, John Stoffel, MD, J Ouentin Clemens, MD MS

University of Michigan, Ann Arbor, MI

(Presented by: Sara Lenherr, MD)

Introduction: OnabotulinumtoxinA (BTXA) injection is frequently used to manage neurogenic detrusor overactivity (NDO). Despite published trial protocols, large amounts of variability in injection parameters exist. Our aim was to determine whether certain injection parameters, such as BTXA concentration and number of injection sites, affects clinical outcomes.

Methods: A retrospective chart review was performed of patients who underwent BTXA injection in our center from May 2002 to September 2013 with 8 surgeons. Information on the indication, dose, concentration, number of injection sites, and adverse events were collected. Procedural failures were defined by the following criteria: the need for repeat injection, increase in oral medication regimen or an additional procedure within 3 months of BTXA injection. Injections to the external urethral sphincter or bladder neck were excluded. Statistical analysis was performed with Fischer's exact test.

Results: One-hundred forty-four unique patients had a diagnosis of NDO and underwent at total of 425 BTXA injections; specifically 6, 113 and 295 injections were performed with 100U, 200U and 300U of BTXA, respectively, in varying concentrations and numbers of injection sites. Of the 425 total injections, 47\% were trigone-sparing intradetrusor injections. While number of BTXA units was specified for all injections, only $57 \%(n=242)$ of the injections also listed the dilution volume and number of sites. For analysis of success, injections were limited to those with standard injection variables, as listed in Table. Successful therapeutic effect was reported in $78 \%(n=171)$ of those injections. In contrast, $12 \%(n=26)$ were failed injections. The remainder of injections $(n=23)$ did not have complete follow-up for analysis. Statistical analysis showed that concentration and number of sites did not impact efficacy of $200 \mathrm{U}$ or $300 \mathrm{U}$ injections ( $\mathrm{p}>0.05$ ). There were a total of 14 adverse events for all BTXA injections $(n=425)$ including 4 (1\%) post-procedure UTI/urosepsis and $3(<1 \%)$ gross hematuria requiring clot evacuation.

Conclusion: For patients with NDO, intravesical BTXA concentration and number of injection sites did not affect clinical outcome.

Clinical Relevance: Neurogenic Bladder

\begin{tabular}{|c|c|c|c|}
\hline & $\begin{array}{l}\text { Failed treatment } \\
N=26(12 \%)\end{array}$ & $\begin{array}{l}\text { Successful treatment } \\
\qquad N=171(78 \%)\end{array}$ & p-value \\
\hline \multicolumn{4}{|l|}{$100 U$ BTXA } \\
\hline 10 units per site (100U/ $10 \mathrm{cc}$ in 10 sites) & 0 & $2(100 \%)$ & - \\
\hline \multicolumn{4}{|l|}{$200 U$ BTXA } \\
\hline 10 units per site $(200 \mathrm{U} / 20 \mathrm{cc}$ in 20 sites $)$ & $6(18 \%)$ & $27(82 \%)$ & 1.0 \\
\hline 20 units per site (200U/ $10 \mathrm{cc}$ in 10 sites) & $6(19 \%)$ & $25(81 \%)$ & \\
\hline \multicolumn{4}{|l|}{$300 U$ BTXA } \\
\hline 10 units per site ( $300 \mathrm{U} / 30 \mathrm{cc}$ in 30 sites) & $3(7.7 \%)$ & $36(92.3 \%)$ & 0.55 \\
\hline 30 units per site (300U/ $10 \mathrm{cc}$ in 10 sites) & $11(12 \%)$ & $81(88 \%)$ & \\
\hline
\end{tabular}

\section{Poster \#M25}

\section{OUTCOME OF SACRAL NEUROMODULATION (SNM) IN POST RADICAL PROSTATECTOMY REFRACTORY OVERACTIVE BLADDER (OAB)}

Stephen Mock, MD, David J. Osborn, MD, Anne Scott, MD, Melissa Kaufman, MD, Roger R. Dmochowski, MD, W. Stuart Reynolds, $M D$

Vanderbilt University, Nashville, TN

(Presented by: Stephen Mock, MD)

Introduction and Objectives: Radical prostatectomy is an effective treatment for localized prostate cancer but can result in long term complications such as urinary incontinence and erectile dysfunction. While post prostatectomy incontinence is usually stress predominate, urge associated incontinence (UUI) can be present. SNM has proven efficacy in refractory OAB and UUI but there is scant data available in the post radical prostatectomy setting. The aim of this study is to determine the outcome of SNM on post prostatectomy refractory $\mathrm{OAB}$ and UUI.

Methods: A total of 490 SNM were performed between 2000 and 2012. One hundred and twenty six $(26.4 \%)$ were male. Of this, $13(10.3 \%)$ men had a prior history of either open radical retropubic prostatectomy (RRP) or robotic assisted laparoscopic prostatectomy (RALP). The indications for SNM were refractory UUI in all cases. Urinary morbidity was recorded using the International prostate symptom score (IPSS) pre SNM and at last follow-up. Success was defined as improvement in IPSS of 6 or greater. Failure was defined as IPSS improvement less than 6 , worsening of IPSS, or explantation of device due to lack of efficacy.

Results: Ten patients were eligible for analysis. Median age at SNM was 70 years old. Eight men had RRP and 2 had RALP. Median time from prostatectomy to SNM was 73.5 months (range 22 to 178 months). Median follow-up after SNM was 22 
months (range 1 to 64 months). Five of 10 men reported clinical stress urinary incontinence (cSUI) in addition to UUI. Urodynamic evaluation was available in 8 of 10 patients. Detrusor over-activity was demonstrable in all patients and 2 had SUI. Seven of ten $(70 \%)$ men had success from SNM. Responders had a higher pre SMN IPSS than non-responders (21 vs. 13, $p=0.03$ ). IPSS at last follow-up was lower for responders than nonresponders but did not meet significance ( $11 \mathrm{vs.} 18, \mathrm{p}=0.08$ ). Univariate analysis for variables that may predict success was not significant for age, presence of diabetes, history of psychiatric disease, history of spinal surgery, history of neurological disease, or presence of cSUI.

Conclusion: Refractory $\mathrm{OAB}$ post radical prostatectomy is not common. SNM in these cases provide efficacy similar to that of the non prostatectomy population. The presence of severe symptoms may be a factor in predicting outcome. Though the study was small in number, there is a dearth of available literature on this subject.

Clinical Relevance: LUTS / Voiding Dysfunction

\section{Poster \#M26}

\section{ADVANCE MALE SLING: PRE-OPERATIVE PAD WEIGHT AS A PREDICTOR OF SURGICAL OUTCOME}

Casey Kowalik, MD, Christopher Lebeis, MD,

Arthur Mourtzinos, MD, MBA

Burlington, $M A$

(Presented by: Casey Kowalik, MD)

Introduction: The AdVance male sling has been advocated for use in patients with mild to moderate urinary incontinence. Patients with severe urinary incontinence (24-hour pad usage $\geq 6$ per day or pad weight $\geq 400$ grams) have had reported cure following sling placement. The aim of this study is to determine whether pre-operative pad weight is an indicator of surgical outcome.

Methods: We prospectively evaluated 56 patients undergoing placement of an AdVance male sling for urinary incontinence following prostate surgery. Cure was defined as no pad usage or wearing one pad for security only as it remained dry. Improvement was defined as $\geq 50 \%$ reduction in pad weight. Failure was defined as $<50 \%$ decrease in pad weight or the need for salvage therapy. Patients were assessed by pad count, pad weight, and self-assessment through IIO and UDI scores at baseline and during follow-up, as well as reporting a percentage improvement following surgery. One patient was excluded as no post-operative pad weight was available.

Results: At a median follow-up of 33 months, 21 patients (38\%) had pad weight 0-100 grams (g), 21 (38\%) had pad weight between 101-200g, 9 (16\%) with pad weight between 201$300 \mathrm{~g}$, and 4 patients (7\%) with pad weight $>300 \mathrm{~g}$. Of the 21 patients with pad weight $<100 \mathrm{~g}, 95 \%(n=20)$ were cured following sling placement. Of the patients not cured, median pre-operative pad weight was $252 \mathrm{~g}$ versus $98 \mathrm{~g}$ in those who were cured. The likelihood of sling failure increased significantly with pre-operative pad weight exceeding $200 \mathrm{~g}$ ( $p$ $=0.0067$ ) with a $54 \%$ failure rate (7/13). In patients with preoperative pad weights $>200 \mathrm{~g}$, post-operative IIO and UDI scores were significantly different from patients with pad weight $<200$ g with $p=0.02$ and $p=0.01$, respectively. Patient reported percentage of improvement was also significantly different between patients with pad weight $<200 \mathrm{~g}$ and those with pad weight $>200 \mathrm{~g}(\mathrm{p}=0.02)$. Five of the 13 patients with pre-operative pad weights $>200$ grams went on to have salvage procedure compared to 1 patient in the group with pad weight $<200$ g.

Conclusion: Patients with pad weight $>200$ g were more likely to fail and reported less subjective improvement compared to patients with pad weight $<200 \mathrm{~g}$. When offering the AdVance male sling to patients with pre-operative pad weight $>200$ grams, patients should be counseled regarding the increased rate of failure.

\section{Poster \#M27}

\section{ASSESSING VARIABILITY OF THE 24-HOUR PAD WEIGHT TEST IN MEN WITH POST-PROSTATECTOMY INCONTINENCE}

Rena D. Malik, MD, Joshua A. Cohn, $\mathbf{M D}^{1}$, Paula Fedunok MMS, PA-C $C^{1}$, Doreen E. Chung, $M^{2}$ and Gregory T. Bales, $\mathrm{MD}^{1}$

${ }^{1}$ University of Chicago Medical Center, Chicago, IL; ${ }^{2}$ Mount Sinai Hospital, University of Chicago Medical Center, Chicago, IL (Presented by: Rena D. Malik, MD)

Introduction: The surgical management of post-prostatectomy incontinence (PPI) is challenging. The 24-hour pad weight test is commonly used to objectively quantify post-prostatectomy incontinence. The overall gram weight amount of leakage may help guide male sling (MS) versus artificial urinary sphincter (AUS) surgical intervention. Previous literature has suggested that men with $<100 \mathrm{~g} /$ day and $>400 \mathrm{~g}$ /day leakage are best suited for MS and AUS, respectively. The pad weight test's reliability and variability in the post-prostatectomy patient has not been evaluated

Methods: 24 patients who underwent radical prostatectomy were prospectively enrolled. Patients demonstrated persistent stress urinary incontinence without significant urge urinary incontinence symptoms or previous history of incontinence surgery. Eligible men performed 24-hour pad weight tests along with a short survey documenting activity level and number of pads used on 3 consecutive alternating days.

Results: In general, pad weights over three days were wellcorrelated for each patient (ICC 0.85 [95\% CI 0.73-0.93], $p<0.001)$. However, based on previous literature, treatment may have been altered in $50 \%(12 / 24)$ of patients depending on the day 24-hour pad weight was collected. The mean change in leakage for a one-point increase in activity level was $120.4 \mathrm{~g}$ (95\% CI -81.6-159.1, $\mathrm{p}<0.001)$.

Conclusion: Patients may have an altered treatment recommendation (MS vs. AUS) based on daily variation in 24-hour pad weight. Variation in pad weights is associated with changes in activity level. It is important to quantify patient's activity level on the day of a 24-hour pad weight with the expectation that pad weight can increase significantly with increase in activity. Multiple 24-hour pad weight tests may also be beneficial. Further studies are needed to better characterize PPI and stratify patients for surgical treatment.

Clinical Relevance: Male Incontinence

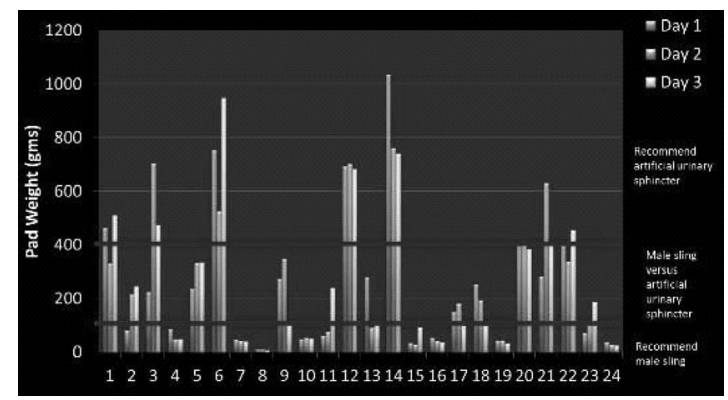




\section{Poster \# M28}

SYMPTOM PERSISTENCE IN A COMMUNITY COHORT OF WOMEN WITH INTERSTITIAL CYSTIITIS/BLADDER PAIN SYNDROME (IC/BPS): 3, 6, 9, AND 12 MONTH FOLLOW UP FROM THE RICE COHORT

Anne M. Suskind, $M D, M S^{1}$, Sandra H. Berry, $M A^{2}$, Marika J. Suttorp, $\mathrm{MS}^{2}$, Marc N. Elliott, PhD, $\mathrm{MA}^{2}$ and J. Ouentin Clemens, MD, $\mathbf{M S}^{1}$

${ }^{1}$ University of Michigan, Ann Arbor; ${ }^{2}$ RAND, Santa Monica, CA (Presented by: Anne M. Suskind, MD, MS)

Introduction and Objectives: To describe persistence of interstitial cystitis/bladder pain syndrome (IC/BPS) symptoms and to identify factors associated with this persistence in a population-based cohort over the course of 12 months.

Methods: A probability sample of US women was identified through a two-stage telephone screening process using the RICE high sensitivity case definition. A randomly selected subgroup of these women $(n=508)$ were enrolled in a longitudinal study where they were interviewed about their symptoms at baseline, three, six, nine and 12 months. Bivariate and multivariate linear regression analyses were performed to determine predictors of persistence of symptoms over all four waves of data collection.

Results: A total of 436 women (86\%) provided non-missing data in all 4 waves and were included in the analysis. The mean age of women at baseline was 47.5 years, $79 \%$ were white and $73 \%$ had at least some college education. Forty-one percent of women met the RICE high sensitivity case definition for IC/BPS at baseline and in all 4 waves, and an additional 21\% met the definition at baseline and in 3 waves. Selfpast continuity of symptoms at baseline was a strong predictor of symptom persistence over 12 months $(p<0.01)$. Younger age and higher scores on the O'Leary-Sant Interstitial Cystitis Symptom Index at baseline were also significant predictors of persistence (p's $<0.01$ ), while lower levels of education were associated with less persistence $(p=0.02)$. Degree of pelvic pain at baseline was not an independent predictor of persistence of IC/BPS symptoms $(p=0.25)$.

Conclusions: We have previously reported that $6.53 \%$ of U.S. women report symptoms of IC/BPS based on the RICE high sensitivity definition. The current analysis demonstrates that the majority of these women report that their symptoms persisted across 3 to 4 waves at three-month intervals over a 12 month period of time. These women tended to be younger and tended to report both a history of continuity of symptoms and higher severity of symptoms at baseline.

Funding: NIDDK U01DK070234-01 and NIH/NIDDK Grant T32 DK07782

Clinical Relevance: IC and Pelvic Pain-UTI / Inflammatory

\section{Poster \#M29}

FACTORS THAT INFLUENCE THE DECISION FOR SURGERY IN PATIENTS WITH BENIGN PROSTATIC HYPERPLASIA: ARTIFICIAL NEURAL NETWORK ANALYSIS

Myong Kim, $\mathrm{MD}^{1}$, Changwon Yoo, $\mathrm{PhD}^{2}$, Jae-Seung Paick, $M D, \mathrm{PhD}^{1}$ and Seung-June $\mathrm{Oh}, \mathrm{MD}, \mathrm{PhD}^{1}$ ${ }^{1}$ Department of Urology, Seoul National University Hospital, Seoul, Korea; ${ }^{2}$ Department of Biostatistics, School of Public Health, Florida International University, Florida

(Presented by: Myong Kim, MD)

Objectives: Besides absolute surgical indications, there are substantial gray zone in relative surgery indication in the treatment for LUTS/BPH. There are many factors that influence surgical decision in real life clinical practice. We aimed to identify predictive factors for surgical decisions for BPH by establishing statistical models based on the accumulated data from expert urologists.

Methods: The database of 1,089 consecutive patients with LUTS/BPH who were treated by the two physicians (SJO, JSP) from Oct 2004 to Dec 2012, was retrospectively reviewed. Among them, patients with UTI, neurogenic bladder, previous genitourinary (GU) surgery, GU malignancy, or incomplete data set were excluded. International Prostatic Symptom Score (IPSS), free flow uroflowmetry (UFM), post-void residual volume (PVR), prostate specific antigen (PSA), total prostate volume (TPV) and urodynamic study (UDS) parameters were selected for two prediction models: logistic regression (LR) model and artificial neural network (ANN) model. For ANN model, patients were randomly divided into training (70\%) and testing (30\%) sets.

Results: A dataset of 702 patients was finally analyzed. Mean age was 66.7 ( $\pm 7.1, S D$ ) years and TPV, PSA, IPSS total, IPSS QoL, Omax, PVR and bladder outlet obstruction index (BOOI) were $49.2( \pm 23.3) \mathrm{ml}, 2.76( \pm 3.50) \mathrm{ng} / \mathrm{ml}, 18.2( \pm 7.7), 4.0( \pm 1.1), 11.9$ $( \pm 5.8) \mathrm{ml} / \mathrm{sec}, 59.8( \pm 78.7) \mathrm{ml}$ and $35.3( \pm 23.9)$. Among them, 414 patients (58.9\%) ultimately received BPH surgery. In LR model, TPV $(\beta=0.025)$, IPSS OoL $(\beta=0.395)$, Omax $(\beta=-0.040)$, BOOI $(\beta=0.024)$, physician $(\beta=-1.403)$ were predictive variables. In ANN model, the predictive performance was the highest at 12 hidden nodes. The overall sensitivity, specificity and accuracy of LR model for surgical decision prediction were $84.3 \%, 51.3 \%$ and $71.1 \%$, and those of ANN model were $88.4 \%$, $41.3 \%$ and $69.7 \%$, respectively. Area under ROC curve of both model was same (0.730).

Conclusion: Our results demonstrated that BOOI, TPV and IPSS OoL were the most predictive factors for surgical decision for BPH patients in real life practice. Further studies including more broad variables are needed to establish clearer guideline for decision in LUTS/BPH patients.

\section{Poster \# M30}

IC / BPS PATIENTS WITH BLADDER MUCOSAL CRACKS: WHO ARE THEY AND CAN THEY BENEFIT FROM CORTICOSTEROID TREATMENT?

Darlene Morrissey, DO, Dominique El-Khawand, MD, Peter O'Hare, III, MD, Lauren Rittenberg, MPH, DO and Kristene Whitmore, $\mathrm{MD}$

Drexel University College of Medicine, Philadelphia, PA

(Presented by: Darlene Morrissey, DO)

Introduction: There is little data on the etiology of bladder mucosal cracks in patients with interstitial cystitis/bladder pain syndrome (IC/BPS). The primary objective of this study is to evaluate the effectiveness of treating bladder mucosal cracks with submucosal Kenalog injections.

Methods: This is a retrosepective cohort of patients with IC/BPS and bladder mucosal cracks found at the time of bladder hydrodistension from December 2009 to June 2012. A 200mg Kenalog suspension was injected submucosally into the base and edges of the cracks. Data collected at baseline and 12 weeks postoperatively included demographics, urodynamic findings, validated questionnaires including the interstitial cystitis symptom and problem indicies (ICSI/ICPI), the female sexual function index (FSFI), the pelvic floor impact questionnaire (PFIO) for the bladder and the visual analog scale for pain associated with daily activity (VAS-D). 
Results: A total of 100 patients were included. Sixty percent $(n=60)$ of the patients were sexually active with $36 \%(n=22)$ reporting dyspareunia and a mean FSFI score of $24.95(n=45$; $\mathrm{SD} \pm 19.1$ ). Pre-operative questionnaires also revealed a baseline mean ICPI of $10.44(n=64 ; S D \pm 4.1)$, ICSI of $10.11(n=64$; $\mathrm{SD} \pm 4.7)$ and bladder PFIO of $46.4(\mathrm{n}=40 ; \mathrm{SD} \pm 32.2)$. A total of 69 patients underwent urodynamics pre-operatively with a mean bladder capacity of $429 \mathrm{ml}(S D \pm 214 \mathrm{ml})$ and $45 \%(n=32)$ had a diagnosis of sensory urgency. Mean maximum anesthetic cystometric capacity (MACC) was $788.8 \mathrm{ml}$ (SD \pm 246.6$)$. Compared to baseline, the mean VAS-D score showed a nonstatistically significant decrease from 5.1 to 4.0 at 12 weeks post-op ( $p=0.435)$ while there was a significant decrease in urinary frequency from a mean of 11.7 to 9.1 daily episodes $(p=0.05)$ and nocturia from a mean of 3 to 1.6 nightly episodes $(p=0.008)$. There was a negative correlation between VAS-D and MACC (Rho 0.34; $p=0.003$ ). Conversely, a postive correlation was found between ICPI score and pre-operative maximal urethral closure pressure (Rho $0.4 ; p=0.02$ ).

Conclusions: This subset of IC/BPS patients who have bladder mucosal cracks appear to have moderate symptom severity scores. The use of intramural corticosteroid injections may be beneficial to symptom control and improvement in the quality of life of patients with IC/BPS by decreasing urinary frequency and nocturia.

Clinical Relevance: IC and Pelvic Pain-UTI / Inflammatory

\section{Poster \#M31}

\section{OFFICE-BASED TRANSVAGINAL VESICOVAGINAL FISTULA REPAIR}

Jeffrey Tomasini, MD, Nissrine Nakib, MD

University of Minnesota, Minneapolis, MN

(Presented by: Jeffrey Tomasini, MD)

Introduction: Vesicovaginal fistulas (VVF) can develop following obstetric trauma, pelvic or vaginal surgery, or radiation therapy. They result in urinary incontinence and recurrent urinary tract infections. Conservative therapy is often not curative, and surgical resection of the fistula tract can be invasive. We present two cases of VVF treated with local injection of an acellular matrix product in an office-based setting.

Objectives: To report the outcomes of transvaginal VVF repair with MatriStem porcine urinary bladder micromatrix (ACell Inc., Columbia, MD, USA) in an office-based setting. No financial funding was utilized in this case report.

Methods: Retrospective chart review was performed on two patients with a single, small $(3 \mathrm{~mm})$ vesicovaginal fistula that developed following gynecologic surgery. Patients underwent injection of the vesicovaginal fistula tract and surrounding bladder mucosa in an office setting.

Results: Two patients underwent above described procedure in clinic. Cystography 3 weeks after the procedure demonstrated closure of the fistula tract. A single-dye pad test was performed to confirm these findings given small fistula size and was negative in both patients. Both women had resolution of continuous urinary incontinence.

Conclusions: MatriStem micromatrix appears to be a promising product in the treatment of small vesicovaginal fistulas, and a potential alternative to more invasive procedures. Additionally, it can safely and readily be performed in an office-based setting.

Clinical Relevance: Reconstruction

\section{Poster \#M32}

\section{OUTCOMES OF SACRAL NEUROMODULATION FOR} OVERACTIVE BLADDER IN OCTOGENERIAN FEMALES

Eugene W. Lee, $\mathrm{MD}^{1}$, Justina Tam, $\mathrm{BS}^{2}$, Chong H. Choe, $\mathrm{MD}^{1}$, Una Lee, $M^{1}$, Alvaro Lucioni, $M^{1}$ and Kathleen Kobashi, $M^{1}$ ${ }^{1}$ Virginia Mason Medical Center, Seattle, WA; ${ }^{2}$ Stony Brook University School of Medicine, Stony Brook, NY

(Presented by: Eugene W. Lee, MD)

Introduction: Elderly women have a high incidence of overactive bladder (OAB) refractory to medical therapy. Clinicians may be reluctant to offer sacral neuromodulation (SNM) particularly in octogenarians, due to the comorbidities and perceived potential for difficulty understanding the device. We investigated outcomes of SNM in women $\geq 80$ years of age.

Methods: From 2000 to 2013, 24 octogenarians with OAB underwent stage I implantation of the Interstim SNM device and were followed in a prospective longitudinal database. Per standard convention, we defined success of stage I as greater than 50\% improvement in incontinence episodes and/or pad use after the testing period, and these patients underwent placement of an implantable pulse generator (IPG). Patients were followed postoperatively at office visits and with mailed questionnaires at 6 and 12 months, then yearly thereafter. The primary outcome was the rate of stage I success, and secondary outcomes were complication rate and patientreported improvement/satisfaction.

Results: 24 octogenarians were identified with median age 84.5 years (range 80-89). Based on a median Charlson comorbidity index of 5 (range 4-8), average 10 year expected life expectancy was 22\%. Mean BMI was 27.8 (range 16-36), $25 \%$ were diabetic, and 3 patients had suffered from stroke with no other neurologic diseases. 35\% had detrusor overactivity and $50 \%$ had urgency-predominant mixed incontinence. 2 were deceased at last follow-up. 18 (75\%) patients experienced stage I success with greater than $50 \%$ improvement in $\mathrm{OAB}$ symptoms and had an IPG placed. At mean follow-up of 14 months (range 1-58), 11 of the 18 (61\%) had continued success. There were no Clavien grade 3 complications. 3 (17\%) patients lost therapeutic effect of which 2 regained efficacy after lead revision. Of 9 patients returning the PGI-I questionnaire at mean follow-up of 18 months, 7 (78\%) reported that their condition was improved, and 2 reported no change. 56\% of patients reported satisfaction.

Conclusions: In our series of SNM in octogenarians, which to our knowledge is the largest reported in the literature, there was a high rate of stage I success with no major complications. Moreover, a majority report improvement and satisfaction at a mean follow-up of 18 months. Our findings demonstrate good results in this population and therefore advanced age should not preclude consideration of SNM as a treatment option in the elderly.

Clinical Relevance: Neuromodulation

\section{Poster \#M33}

OPEN VERSUS ROBOTIC-ASSISTED LAPAROSCOPIC AUGMENTATION ILEOCYSTOPLASTY AND MITROFANOFF APPENDICOVESICOSTOMY (RALIMA) FOR THE MANAGEMENT OF NEUROGENIC BLADDER IN OLDER CHILDREN AND ADOLESCENTS

Joshua Cohn, MD, Prithvi Murthy, BS, Pankaj Dangle, MD, Gregory Bales, MD, Mohan Gundeti, MD

University of Chicago, Chicago, IL

(Presented by: Joshua Cohn, MD) 
Introduction and Objectives: Augmentation ileocystoplasty (AI) may be required for refractory neurogenic bladder. We aimed to compare perioperative and long-term outcomes associated with an open versus minimally-invasive approach to AI in older children and adolescents.

Methods: All patients undergoing RALIMA or open AI within our institution between 2/08 and 10/12 were reviewed. Data obtained on each patient included baseline demographics, urodynamics, perioperative and hospital data, and short, intermediate, and long-term outcomes.

Results: The RALIMA cohort consisted of 15 patients and the open 14. Median follow-up was longer for the RALIMA group (43.7 vs. 18.1 months, $p=0.06$ ). The RALIMA cohort was significantly older ( 11.5 vs. 7.3 years, $p=0.05$ ) but did not differ significantly with respect to gender, preoperative DLPP or percent of expected bladder capacity (expected $=$ age $/ 2+6$ ) (Table). There were no significant differences in concomitant procedures performed. Mean operative time (incision to closure) was significantly longer in the RALIMA cohort (700 vs. 388 minutes, $p<0.001$ ). 2 RALIMAs were converted to open (Monti channel creation). There was a trend towards decreased length of stay and IV morphine use in the RALIMA cohort (6.8 vs. 15.6 days, $p=0.08 ; 1.0$ vs. $2.5 \mathrm{mg} / \mathrm{kg}, \mathrm{p}=0.21$ ). Both groups achieved significant increases in bladder capacity. In the first 30 days postoperatively, significantly fewer patients experienced a complication of any severity in the RALIMA cohort ( $40 \%$ vs. $86 \%, p=0.02$ ), but there was no significant difference observed beyond 30 days. Since surgery, 7 RALIMA (47\%) and 10 open patients $(63 \%)$ have required minor procedures for stoma revision or cystoscopy, and $4(27 \%)$ and $2(14 \%)$, respectively have required major procedures (RALIMA: 3 bladder neck closures, 1 cystectomy; open AI: 1 ex-lap, 1 takedown of colon and bladder stoma).

Conclusion: RALIMA is associated with increased operative time but may decrease 30-day morbidity without apparent compromise in long-term outcomes, and its use should be considered in adults. However, long-term morbidity remains significant, and patients should be counseled regarding the likelihood of need for future procedures regardless of approach. Clinical Relevance: Neurogenic Bladder
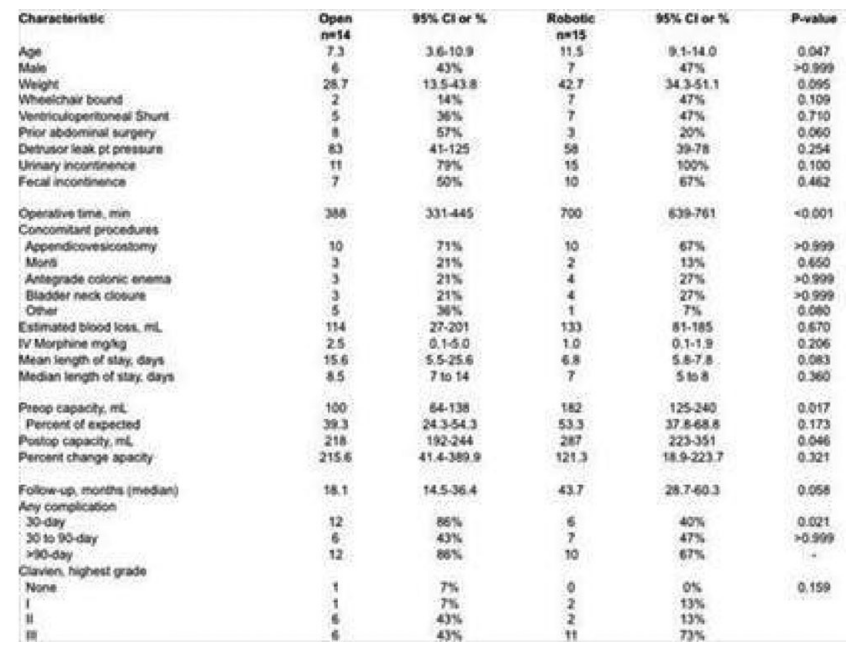

Poster \#M34

CONCOMITANT AUTOLOGOUS FASCIA PUBOVAGINAL SLING AT THE TIME OF TRANSVAGINAL MESH REMOVAL RESULTS IN DECREASED PAD USAGE VERSUS DELAYED AUTOLOGOUS SLING IN PATIENTS WITH MESH RELATED COMPLICATIONS
Taylor Vaughan, MD, S. Walker Nickles, MD, Eric Rovner, MD Medical University of South Carolina, Charleston, SC (Presented by: Taylor Vaughan, MD)

Introduction and Objectives: Patients with persistent incontinence or complications after transvaginal (TV) mesh placement are presenting to urologists with increasing frequency. Many of these patients desire TV mesh removal and further anti-incontinence intervention. We compared performing autologous fascia pubovaginal sling (AFPVS) concomitantly with mesh excision, versus delaying AFPVS after TV mesh excision.

Methods: We retrospectively analyzed patients with history of TV mesh and subsequent excision who had AFPVS from 2007 through March 2013. Indications for AFPVS included obstruction $(n=14)$, unimproved incontinence $(n=37)$, mesh extrusion $(n=10)$, or erosion $(n=12)$. All patients underwent video urodynamics (VUDS), cystoscopy, pad quantity and 24 hour pad weights. Quality of life (QOL) was assessed using a 7 point, subjective Likert scale.

Results: 39 patients met inclusion criteria. 26 patients underwent AFPVS concomitantly with TV mesh removal, and 13 patients underwent delayed AFPVS at a median 7 months (range 3 to 22 months) after TV mesh removal. Patients in the concomitant group were significantly older vs those in the delayed group (mean 59 vs 47 years, $p<0.05$ ). There was no difference in number of prior TV mesh procedures (1.4 vs 1.5, $p=0.71$ ). Preoperative dyspareunia, pad number, pad weight, and VUDS parameters were also statistically similar. Preoperative erosion into the GU tract was less common in the concomitant vs the delayed group ( $19 \%$ vs $54 \%, \mathrm{p}<0.05$ ); however, mesh extrusion rates were similar (23\% vs $31 \%$, $\mathrm{p}=0.62$ ). Concomitant AFPVS patients were less likely to require Martius flap intraoperatively vs delayed patients $(0 \%$ vs $31 \%, p<0.01)$. Concomitant AFPVS patients experienced greater relative pad reduction vs delayed ( $92 \%$ vs $71 \%, p<0.05)$ with similar rates of patients requiring clean intermittent catheterization ( $31 \%$ vs $23 \%, p=0.63$ ). Intraoperative complication rates ( $8 \%$ vs $15 \%$ ) and need for further GU procedures (23\% vs $31 \%$ ) were also similar. Subjective postoperative OOL was similar with median response being "delighted"in both groups.

Conclusions: In our series, AFPVS was a successful option for patients with persistent incontinence or complications after TV mesh placement. Those undergoing concomitant repair had a significantly lower likelihood of requiring Martius flap and had a significantly greater reduction in relative pad usage vs those undergoing delayed autologous sling, while avoiding an additional anesthetic.

Clinical Relevance: Reconstruction

\section{Poster \#M35}

\section{6-YEAR TRENDS IN VAGINAL MESH COMPLICATION AT A TERTIARY ACADEMIC CENTER}

Austin Younger, MD, Lara Maclachlan, MD, Kelly Johnson, MD, Justin Ellett, MD, Gini Ikwuezunma, BS, Michelle Koski, MD, Ross Rames, MD, Eric Rovner, MD

Medical University of South Carolina, Charleston, SC (Presented by: Austin Younger, MD)

Introduction and Objectives: Until recent years, the number of surgeries utilizing transvaginal mesh had been steadily increasing. The FDA statements in 2008 and 2011 regarding complications of transvaginal mesh may have likely slowed 
the growth of such implantation cases. However, it is unclear whether surgeries for mesh explantation are increasing. The objective of this study is to evaluate for trends in mesh removal surgery over the last 6 years.

Methods: We retrospectively analyzed 191 patients who underwent explantation of transvaginal mesh from 8/2007 until 8/2013. Indications for mesh removal, operative approach and need for subsequent surgeries were investigated.

Results: Indications for transvaginal mesh removal overlapped in many patients and included: bladder outlet obstruction $(65 \%)$, erosion (15\%), exposure (30\%), pain (39\%), recurrent urinary tract infections (22\%) and de novo lower urinary tract symptoms (11\%). Regardless of the indication, we observed an increasing trend in the number of mesh explantation procedures performed at our institution. In addition, 38 of 191 patients $(20 \%)$ required subsequent genitourinary procedures following mesh explantation and of these 21 out of 38 (55\%) had presented with erosion or mesh exposure.

Conclusions: This study demonstrates a steadily rising incidence of surgical intervention for mesh related complications over the past 6 years at a tertiary care center. There was a near doubling of cases observed after the FDA released an updated statement regarding mesh safety in July of 2011. This trend could be related to changes in referral patterns, an increased awareness and recognition of potential mesh complications, an actual increase in mesh surgery implantation, an actual increase in mesh complications or other factors.

Clinical Relevance: Female Urology-including Incontinence

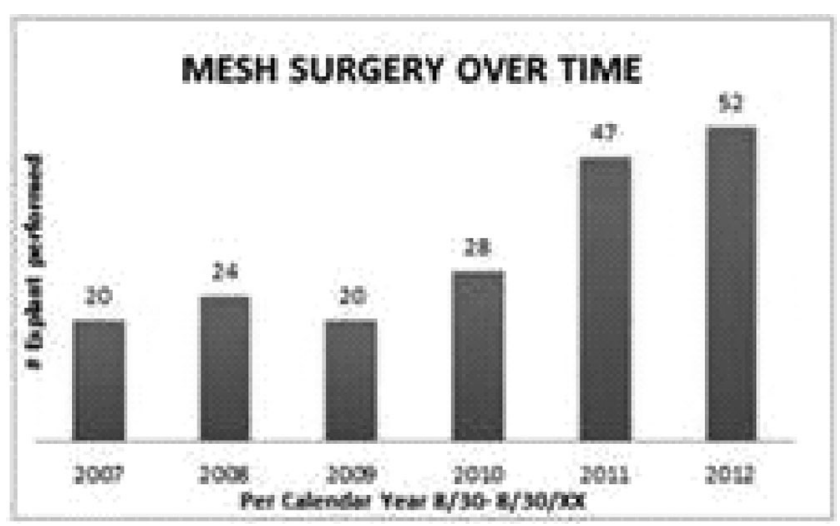

Poster \#M36

WHO REPORTS SLING AND MESH COMPLICATIONS TO THE UTILIZATION OF THE MANUFACTURER AND USER FACILITY DEVICE EXPERIENCE (MAUDE) DATABASE?

Kristina R. Tzartzeva, Syed A. Hussain, Emily C. Rosenfeld, Chasta D. Bacsu, MD, Jack C. Hou, MD, Alana Christie, Philippe Zimmern, MD

UT Southwestern Medical Center, Dallas, Texas

(Presented by: Philippe Zimmern, MD)

Introduction: The Manufacturer and User Facility Device Experience (MAUDE) database originated in 1991 and is known for its underreporting and difficult access[1]. Mandatory reporting is expected from the industry to allow for fast discovery of medical device malfunctions. Physicians should report as well, either directly or via their industrial partners. In the wake of escalating number of reports prompting 2 recent FDA notifications, we searched for who is reporting information into the system.
Methods: From 2008 to 2012, reports on vaginal mesh and sling complications in the MAUDE database were extracted and analyzed for the reporter occupation and type of device. In addition, the submission process was studied by recording the time required to submit a short series of 11 recent complication referrals.

Results: From a total of 15,452 mesh or sling submitted reports (SR), little change was noted in the number of submissions between 2008-2011. In 2012, the SR numbers increased exponentially, reaching 11,710 . When a reporter occupation was provided, the ratio of lawyers to physicians was 3:1 in 2012 (Figure 1). Ethicon, AMS, and Boston Scientific had the largest numbers of SRs. TVT and Prolift were associated with the greatest number of SRs. After going through the online submission orientation, our average submission time for each report was 16'32"' (11'40 to 20'31).

Conclusions: Physicians are increasingly reporting device complications but less so than lawyers, possibly due to the time-consuming process.

1. FDA U.S. Food and Drug Administration. 05.02.2013 06.09.2013]; Available from: http://www.fda.gov/MedicalDevices/DeviceRegulationandGuidance/PostmarketRequirements/ ReportingAdverseEvents/ucm127891.htm

\section{Number of Reports Submitted Yearly by Selected Sources, 2008-2012}

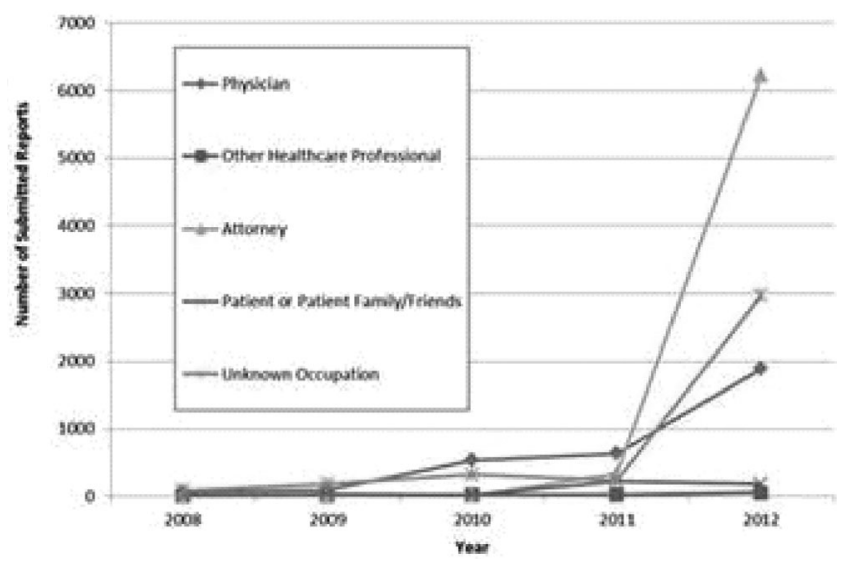

Poster \#M37

BUCCAL MUCOSAL GRAFT URETHROPLASTY FOR THE TREATMENT OF URETHRAL STRICTURE IN THE NEOPHALLUS

Joseph J. Pariser, MD, Joshua A. Cohn, MD, Gregory T. Bales, MD Section of Urology, University of Chicago Medical Center, Pritzker School of Medicine, Chicago, IL

(Presented by: Joseph J. Pariser, MD)

Introduction and Objectives: There are multiple techniques described for neophallus reconstruction for gender reassignment or after traumatic or surgical loss of the phallus. At our institution, the preferred method is by radial forearm free flap. Urethral strictures are not uncommon in these patients. Our preferred approach to strictures refractory to endoscopic management is buccal mucosal graft urethroplasty similar to that used in complex anterior urethral strictures in the standard male phallus. This approach has not previously been well-described in the radial forearm flap neophallus. Herein, we present our experience. 
Methods: Patients who underwent buccal urethroplasty by a single surgeon for urethral stricture in a neophallus between March 1998 and April 2013 were identified. All urethroplasties were performed in a similar manner, using a free buccal mucosal graft ventral onlay following excision of the stricture. Results: The study population consisted of 9 patients. Median followup was 496 days (range 20-). In 7 of 9 patients, neophallus constructions occurred at our institution. The mean age at urethroplasty was 41 years (range 27-56). One patient underwent creation of neophallus following traumatic injury while all others were performed in association with gender reassignment. In all patients, strictures were identified at the anastomotic site to the native urethra. 6 of 9 patients underwent endoscopic management in the form of internal urethrotomy or dilation as first line therapy. Mean stricture length was $3.8 \mathrm{~cm}$ (range 2-6). Following urethroplasty, 4 of 9 patients underwent repeat procedures for treatment of recurrent urethral stricture. 9 total repeat stricture procedures were performed in these 4 patients, including 8 internal urethrotomies and 1 re-do urethroplasty. No patients suffered from any major complications from the procedure.

Conclusion: There is limited experience with urethral strictures in the neophallus with no optimal intervention. Buccal graft urethroplasty affords a safe approach, but recurrences are not uncommon. Modifications in surgical procedures need to be examined and may hopefully achieve more durable, reproducible results.

Clinical Relevance: Reconstruction

\section{Poster \#M38}

YOUTUBE AS A SOURCE FOR VAGINAL MESH INFORMATION

Sarah A. Mitchell, Areeba Sadiq, Nirit Rosenblum, Victor W. Nitti, Benjamin M. Brucker

New York University Langone Medical Center, New York, NY (Presented by: Sarah A. Mitchell)

Introduction and Objectives: Social media networks and websites are an important source of healthcare information exchange. However, the quality of data presented on popular online forums, such as YouTube, is not regulated. Some studies suggest information available online, particularly on controversial topics, may disperse inaccurate information and contribute to public confusion. The goal of this study is to examine information available in YouTube videos on a controversial urologic topic, pelvic organ prolapse (POP) repairs with mesh.

Methods: A keyword search of "vaginal POP repair with mesh"was performed and the first 100 search results examined on $7 / 29 / 13$. Videos not in English, lacking spoken words, or duration $>10$ minutes were excluded. Video characteristics including source (legal, medical, other), number of views, time online and duration were recorded. The content of each video was assessed in the following domains: description of POP, management of POP, explanation of the 2011 FDA Safety Communication, and balanced presentation of information. Scores were assigned based on a series of objective statements made in the video in each domain. Scores were compared in each domain, and significance was assessed by t test.

Results: 51 videos were excluded, and the remaining 49 were viewed. The sources were $69 \%$ legal firm, $24 \%$ medical institution, and $6 \%$ other. The former 2 groups were compared. Legal videos were newer (online for 14.2 vs 27.6 months, $p<0.01$ ), shorter (99 vs 234 seconds, $p<0.01$ ), and trend toward fewer views per month posted (140 vs $279, p=0.20$ ).
The content assessment revealed significantly higher scores in the legal videos for explaining the 2011 FDA Safety Communication (1.5 vs $0.5, p=0.02$ ) and a strong trend toward lower scores in description of POP (0.9 vs $1.8, p=0.05)$. No significant difference between sources was noted in management of POP (1.4 vs $1.8, p=0.27$ ) or balanced presentation (0.4 vs 0.7 , $\mathrm{p}=0.44)$.

Conclusions: The majority of information available in YouTube videos on the topic of vaginal POP repair with mesh is recent, short, and published through legal services that outline the 2011 FDA Safety communication but contain less comprehensive descriptions of POP compared to other videos. However, low overall scores in all domains studied, regardless of source, demonstrate lack of content. This raises questions about the utility of YouTube as a source of information for patients.

Clinical Relevance: Female Urology-including Incontinence

\section{Poster \#M39}

\section{TRENDS IN THE MANAGEMENT OF ELDERLY PATIENTS WITH PELVIC ORGAN PROLAPSE}

Tina Schubert, Melissa A. Laudano, MD, Fujun Zhao, MD, Bilal Chughtai, MD, Richard K. Lee, MD

Department of Urology, Weill Medical College of Cornell

University, New York, NY, USA

(Presented by: Tina Schubert)

Introduction and Objectives: Pelvic organ prolapse (POP) is a common condition in aging women. POP can be treated via surgical or nonsurgical means. We aim to assess nationwide trends in the management of patients treated for POP.

Methods: An analysis of the 5\% Medicare Public Use Outpatient Files (years 2001, 2004, 2007-11) was performed to assess changes in the utilization of transvaginal or transabdominal prolapse repair surgery vs. pessary to treat POP. Patients were identified using International Classification of Diseases, 9th Edition (ICD-9) and the Current Procedure Terminology (CPT) codes. Statistical analyses, including the Fisher and $\chi^{2}$ tests as well as multivariate regression analyses, were performed using SAS 9.3 (SAS Institute Inc., Cary, NC) and SPSS v20 (IBM Corp., Armonk, NY).

Results: A total of 608,680 patients with POP were identified. The percentage of patients undergoing outpatient treatment increased from $7.9 \%$ to $25.3 \%$ over the study period (See Table, $p<0.001$ ). The use of surgery increased from $52.3 \%$ to $73.9 \%$, while the use of pessary decreased correspondingly $(p<0.001)$. Logistic regression analysis showed that older women with POP (OR $=0.59-0.79, \mathrm{p}<0.001)$ were less likely to be treated surgically as were black women compared to whites or other minorities (OR-0.71 vs. $1-1.2, p<0.001$ ).

Conclusions: The treatment of POP has changed over time. A greater percentage of patients are now being treated surgically instead of with pessary. Older women and blacks with POP tend to be treated less often with surgery compared to more conservative measures.

Clinical Relevance: Pelvic Organ Prolapse

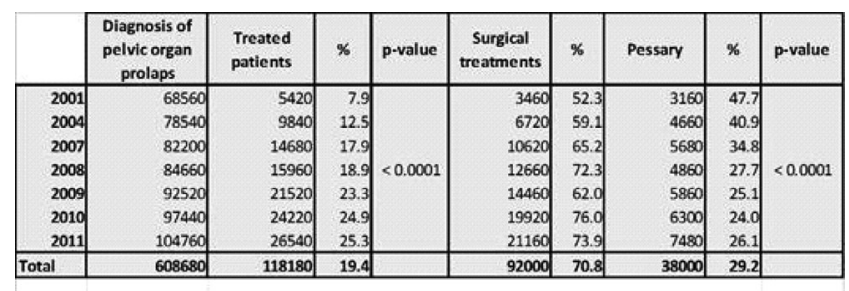

Table: Diagnosis of pelvic organ prolapse and treatment rates 2001-2011 
Poster \#M40

\section{PREVALENCE OF DEFECATORY DYSFUNCTION IN PATIENTS WITH PELVIC ORGAN PROLAPSE: A COMPARTMENT PERSPECTIVE}

Jameca Price, $\mathrm{MD}, \mathrm{MPH}^{1}$, Kara Petrashek, $\mathrm{MD}^{2}$, Sarah McAchran, $\mathrm{MD}^{3}$ and Cynthia Brincat, $\mathrm{MD}, \mathrm{PhD}^{4}$ ${ }^{1}$ Oregon Health \& Science University, Portland, OR; ${ }^{2}$ Medical College of Wisconsin, Milwaukee, WI; ${ }^{3}$ University of Wisconsin Department of Urology; ${ }^{4}$ Loyola University, Chicago, IL (Presented by: Sarah McAchran, MD)

Introduction \& Objectives: The relationship between anatomy and function in the posterior compartment is poorly understood. Our primary aim was to assess the prevalence of defecatory dysfunction (DD) in a urogynecologic population. Our secondary aim was to identify a correlation between DD and Pelvic Organ Prolapse Quantification (POP-O) Bp point $\geq 0$. Methods: We assumed a baseline prevalence of DD in our population to be $20 \%$ based on the literature. We calculated that a sample of 208 subjects with 125 cases $(B p \geq 0)$ and 83 controls were required to detect an increased prevalence of DD at $45 \%$ with $80 \%$ power. ICD -9 codes for prolapse were used to identify subjects seen at an outpatient female urology and urogynecology clinic between 9/2011 and 12/2012. Charts were abstracted for: patient demographics, Bp measurement, and symptoms of defined as any combination of constipation, vaginal splinting to defecate, or sensation of incomplete defecation.

Results: 390 charts were reviewed. 258 subjects met inclusion criteria. Of these, 151 had a $\mathrm{Bp} \geq 0$ and 107 had a $\mathrm{Bp}<0$. There were no statistically significant differences in age, BMI, \#visits, prior hysterectomy or surgery status between groups. In the 151 patients with $\mathrm{Bp} \geq 0,90$ had DD and 61 did not. In the 107 patients with $\mathrm{Bp}<0,41$ had $\mathrm{DD}$ and 66 did not. Of the 258 patients, 131 patients had DD and 127 did not. The only statistically significant difference between those two groups was a slight difference in age. Patients without DD had a distribution of $\mathrm{Bp}$ measurements consistent with those reported in the literature. However, in the DD group, there was more severe posterior compartment prolapse (Figure 1). On multivariate logistic regression analysis only $\mathrm{Bp} \geq 0$ correlated with $\mathrm{DD}$. The odds ratio estimate for $\mathrm{Bp} \geq 0$ in patients with $\mathrm{DD}$ was 2.40 (confidence limits 1.40-4.12).

Conclusions: Our findings suggest that there is a higher prevalence of $\mathrm{DD}$ in the population of patients referred to specialized female pelvic floor clinics than in the general population ( $49 \%$ vs. $20 \%$ ). An even greater prevalence is seen in those patients with a $B p \geq 0$ (59.7\%). We found a positive association between $\mathrm{DD}$ and more severe posterior compartment prolapse as assessed by POP-O measurements.

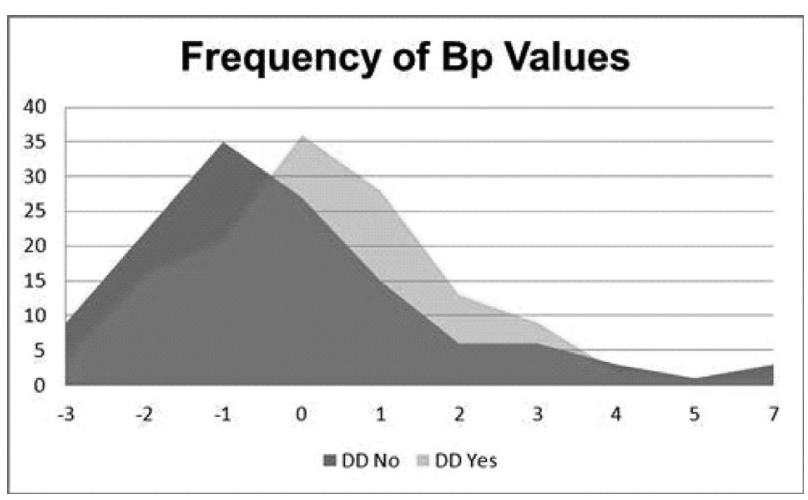

\section{Poster \#M41}

PATIENT WILLINGNESS TO ALLOW HANDS-ON TRAINING FOR POST-GRADUATE LEARNING OF NEW SURGICAL TECHNIOUUES

Emily C. Rosenfeld ${ }^{1}$, Jennifer M. Wimberly, $\mathbf{M D}^{1}$, Alana Christie ${ }^{1}$, Kristina R. Tzartzeva ${ }^{1}$, Syed A. Hussain ${ }^{1}$, Chasta D. Bacsu, $\mathrm{MD}^{1}$, Jack C. Hou, $\mathrm{MD}^{1}$ and Philippe Zimmern, $\mathrm{MD}^{2}$

${ }^{1}$ UT Southwestern Medical Center; ${ }^{2}$ UT Southwestern Medical Center, Dallas, Texas

(Presented by: Philippe Zimmern, MD)

Introduction and Objective: To assess patient willingness to allow hands-on training from the surgeon of record to supplement the current "observership"model when learning new techniques.

Methods: After receiving IRB approval, a survey was administered by neutral third parties to patients in 2 separate outpatient settings. Exclusion criteria included a sub-6th grade reading level, non-English speakers, and pregnancy. Demographic data included age, gender, and race. The survey had three components: the REALM-SF, STAI-X2, and a specifically designed Observer Questionnaire (OQ) with free space for comments. The OO included two questions of interest (Table 1). Results: 99 patients at Location I and 100 patients at Location II met inclusion criteria. $91.9 \%$ of patients at Location I and $82 \%$ at Location II would consent to hands-on training. In regards to current methods of training, responses were: $61 \%$ cadaver lab (A), 63\% training video/reading material (B), $62 \%$ observation without direct contact $(C)$, and $73 \%$ observation with direct trainee contact (D). Age $(p=.41)$ and gender $(p=.42)$ did not significantly affect response, nor did an occupational background in health care ( $p=.55$, surveyed in Location II only). Scores on REALM-SF and STAI-X2 also did not significantly affect responses at either location. The majority of explanations for declining hands-on contact cited unease due to history of past surgical complications.

Conclusion: Supplementing the current "observership" model utilizing "on the job"training can be acceptable to the majority of patients given strict boundaries including informed patient consent, the surgeon of record remaining fully in charge, and the surgeon trainee having tested credentials

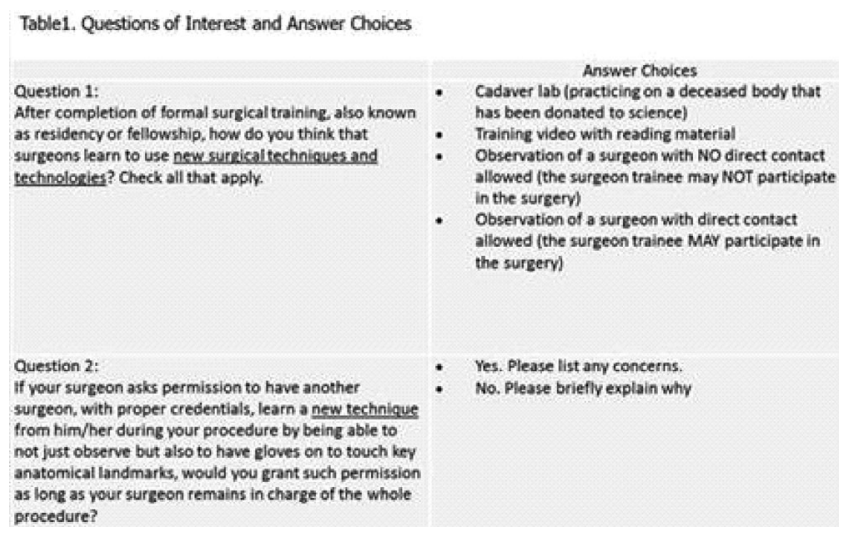

\section{Poster \#M42}

GENERATIONAL AWARENESS OF ERECTILE DYSFUNCTION (ED) AND PELVIC FLOOR DISORDERS (PFD)

Lee A. Richter, $\mathrm{MD}^{1}$, Robert E. Gutman, $\mathrm{MD}^{2}$, Eshetu Tefera $\mathrm{MS}^{3}$, Allison Estep, $\mathrm{BS}^{2}$ and Cheryl B. Iglesia, $\mathrm{MD}^{2}$ 
${ }^{1}$ Medstar Washington Hospital Center/Georgetown University School of Medicine; ${ }^{2}$ Medstar Washington Hospital Center/ Georgetown University School of Medicine, Washington, DC; ${ }^{3}$ Medstar Health Research Institute, Washington, DC (Presented by: Lee A. Richter, MD)

Introduction and Objectives: $\mathrm{ED}$ and $\mathrm{PFD}$ (pelvic organ prolapse (POP), urinary incontinence (UI), fecal incontinence (FI)) negatively impact quality of life, generate significant health-care costs, and increase with age. Approximately $38 \%$ of women between 60-79 years have a symptomatic PFD, similar to the $44 \%$ of men age 60-69 with ED. Despite high prevalence, the general public is poorly informed about PFD as compared to ED. This is the first study to assess Generation X/Y men and women's baseline knowledge of PFD and ED, and to determine whether there are discrepancies in their understanding of the natural history of these conditions.

Methods: An anonymous survey was distributed to community dwelling men and women between the ages of 18-40 using a validated questionnaire to assess familiarity with the epidemiology, pathogenesis, diagnosis, and treatment of POP and UI (Prolapse and Incontinence Knowledge Ouiz), as well as ED. Additional questions captured demographic data, assessed understanding of disease prevalence, and identified sources of knowledge.

Results: Of 226 male and female respondents, $66 \%$ were female and $34 \%$ were male. The majority $(70 \%)$ had completed at least some graduate school. There was a significant difference in baseline knowledge of these conditions, with both men and women scoring highest on the ED and lowest on the POP sections. Men scored significantly higher than women on the ED questions; however, a significant difference in knowledge between men and women was not found for the UI or POP questions (Table 1). Higher education level was associated with a significant increase in knowledge in all domains: UI, POP, and ED. Men and women tended to underestimate the prevalence of PFD and ED, and were worse at estimating the prevalence of PFD as compared to ED.

Conclusion: Both men and women have a worse understanding of POP, as compared to UI or ED, despite high education levels. Generation X/Y women in our study are more familiar with the etiology, prevalence, and treatment for ED than for POP.

Clinical Relevance: Female Urology-including Incontinence

Table 1: Percent Correct
\begin{tabular}{|l|l|l|l|}
\hline & Men & Women & P-value \\
\hline Urinary Incontinence & $87 \% \pm 22$ & $85 \% \pm 20$ & 0.24 \\
\hline $\begin{array}{l}\text { Pelvic Organ } \\
\text { Prolapse }\end{array}$ & $74 \% \pm 34$ & $73 \% \pm 29$ & 0.23 \\
\hline Erectile Dysfunction & $91 \% \pm 16$ & $86 \% \pm 19$ & 0.03 \\
\hline
\end{tabular}

\section{NON-MODERATED POSTERS}

\section{Poster \# NM1}

POSTOPERATIVE VOIDING DYSFUNCTION AND PREDICTORS OF POSTOPERATIVE URINARY RETENTION FOLLOWING PELVIC RECONTSRUCTIVE SURGERY

Kelly Jirschele, $\mathrm{DO}^{1}$, Miriam Seitz, $\mathrm{MD}^{1}$, Alexis Tran, $\mathrm{DO}^{1}$, Lopa Pandya, $\mathrm{MD}^{2}$, Ying Zhou, $\mathrm{PhD}^{3}$

and Adam Gafni-Kane, $\mathrm{MD}^{4}$

${ }^{1}$ University of Chicago/NorthShore Evanston, IL; ${ }^{2}$ University of Chicago Chicago, IL; ${ }^{3}$ NorthShore Center for Biomedical and Research Informatics Evanston, IL; ${ }^{4}$ NorthShore University HealthSystem Evanston, IL

(Presented by: Kelly Jirschele, DO)
Introduction and Objectives: Pelvic organ prolapsed (POP) and stress urinary incontinence are common conditions. Approximately $11 \%$ of women will have surgery for these in their lifetime. Risk factors discussed prior to surgery include voiding dysfunction and postoperative urinary retention (PUR). PUR is the inability to completely empty the bladder after surgery and rates vary greatly. Lack of consistent definitions of voiding dysfunction and PUR make comparison of studies challenging. PUR is bothersome to patients, resulting in decreased patient satisfaction. Although studies suggest variables to predict voiding dysfunction after surgery no consistent predictors exist. The objective of our study was to determine the incidence and risk factors for PUR following pelvic reconstructive surgery. Methods: We conducted a retrospective cross-sectional study. After IRB approval, a list of consecutive surgical patients from $7 / 1 / 11-6 / 30 / 12$ was created from surgical calendar. Electronic medical records were reviewed for demographic, physical and urodynamic information. Chi-square or Fisher's exact, two sample T or Wilcoxon rank sum tests and multivariable logistic regression were used to analyze data.

Results: 418 patients underwent pelvic reconstructive surgery, 396 with complete data were analyzed. 164 (41\%) patients had PUR. 311 (78\%) had a midurethral sling (MUS). Women who had no sling $(O R=0.48 p=0.04)$ or retropubic sling $(O R=0.93 p$ $=0.29$ ) were less likely to develop PUR compared to transobturator sling. Patients who had uterosacral $(O R=2.55 p$ $=0.09)$ and sacrospinous $(\mathrm{OR}=4.41 \mathrm{p}<0.0001)$ apical suspensions were more likely to develop PUR compared to no apical suspension.

Conclusions: $41 \%$ of our patients had PUR. None of the urodynamic parameters reviewed predicted PUR. The odds for developing PUR were different among types of surgical procedures. Our data suggests patients planning apical suspension are more likely to develop PUR than those patients without concomitant apical suspension. MUS is more likely to cause PUR. Our analysis was unable to highlight any clear predictors of PUR; rather it suggests that certain combinations of surgeries will be more likely to result in PUR. This information, with our center's PUR rate of $41 \%$, will be helpful in patient counseling.

Clinical Relevance: Pelvic Organ Prolapse

\section{Poster \# NM2}

\section{POST OBSTRUCTIVE DIURESIS AS A SEOUELA OF URINARY RETENTION: A THING OF THE PAST?}

Steven Weissbart, MD, Rajiv Jayadevan, BA, Karl Coutinho, MD, Luke Hermann, MD and Neil Grafstein, MD Icahn School of Medicine at Mount Sinai, New York, NY (Presented by: Steven Weissbart, MD)

Introduction and Objectives: Postobstructive Diuresis (POD) is a potentially life-threatening sequela of urinary retention (UR). Given the risk of POD, physicians caring for patients with UR often check serial serum chemistries to screen for this potential complication and may recommend hospital admission after bladder drainage. The true incidence of POD is unknown and studies investigating its incidence are outdated, with the majority of data published in and prior to the 1970's (1). We investigate the incidence of POD in patients with UR presenting to the Emergency Room (ER) in 2013.

Methods: From 1/2013 to 9/2013, the records of 168 patients presenting to the ER with UR were identified by chief complaint, diagnosis code and chart review. Parameters analyzed for these patients included: duration of UR, volume 
of retention, serum electrolytes, admission status, urine output and whether a practitioner diagnosed the patient with a POD. For our analysis, we classified a patient as having POD when they met either of the following criteria: (1) practitioner diagnosed patient with POD or (2) patient had electrolyte imbalance consistent with POD (hypernatremia, hyponatremia or hypokalemia) and a daily urine output $>4000$ cc.

Results: Mean age of the 168 patients was 72 years (SD 14) and $86 \%$ were male. Mean duration of UR was 26.7 hours (SD 70) and mean volume of retention was $822 \mathrm{~mL}$ (SD 559). Initial electrolytes were checked in 95 patients (55\%) and their mean serum creatinine was $1.01 \mathrm{MG} / \mathrm{DL}$ (SD 2.5). 45 patients (28\%) had subsequent electrolyte levels checked and their mean serum sodium and potassium levels were $137 \mathrm{MEO} / \mathrm{L}$ (SD 6.8) and 4.4 MEQ/L (SD 0.9), respectively. 130 patients (77\%) were discharged home with an indwelling catheter, had no follow-up diagnosis of POD and did not represent to the ER for electrolyte imbalance. 38 patients (23\%) were admitted to the hospital and none met the criteria for a diagnosis of POD.

Conclusions: Currently, the incidence of POD in patients with UR seems to be exceedingly low. No patient in our series met the criteria for clinically significant POD. Although serial monitoring of electrolytes and hospital admission may be a common tenet in the care of patients after relieving UR, this convention needs reconsideration. The incidence of POD may have decreased over the last decades as patients are seeking earlier medical attention.

(1) Narins R. Post-obstructive diuresis: a review. J Am Geriatr Soc 1970;18:925-36.

Clinical Relevance: LUTS / Voiding Dysfunction

\section{Poster \# NM3}

\section{VOIDING COMPLAINTS IN PATIENTS PRESENTING WITH PELVIC PAIN}

Michael Ehlert, $\mathrm{MD}^{1}$, Larry Sirls, $\mathrm{MD}^{1}$, Donna Carrico, NP, $\mathbf{M S}^{2}$, Emily Dove-Medows, CNM, $\mathrm{MSN}^{2}$, Jason Gilleran, $\mathbf{M D}^{1}$, Jamie Bartley, $\mathrm{DO}^{1}$, Janice Tomakowsky, $\mathrm{PhD}, \mathrm{MPH}^{2}$, Jen Carty, $\mathbf{M A}^{3}$ and Kenneth Peters, $\mathbf{M D}^{1}$

${ }^{1}$ William Beaumont Hospital, Royal Oak, Michigan; ${ }^{2}$ Beaumont Women's Urology Center, Royal Oak, Michigan; ${ }^{3}$ Wayne State University Dept of Psychology, Detroit, Michigan

(Presented by: Michael Ehlert, MD)

Introduction: Women with pelvic pain are heterogeneous and presenting symptoms may not reflect primary organ pathology. The objectives of this study were to determine associations among clinic-demographic variables, and voiding symptoms in women with high pain scores vs. low pain scores who present to a multidisciplinary women's urology clinic.

Methods: A retrospective chart review of consecutive women presenting from July 2012 to April 2013. Data collected include demographics, symptom surveys, past medical history, physical exam findings and final diagnosis codes (ICD-9). Women with higher pelvic pain scores, defined a priori as self-reported severity of $\geq 3 / 10$ in any domain (overall, bladder, vulvar, pelvic), were compared to those with lower pain scores, $<3 / 10$. Voiding symptoms were quantified with Overactive Bladder Questionnaire Short Form (OABq-SF) and Pelvic Floor Distress Inventory (PFDI 20) scores and sub-scores.

Results: 199 women were identified. Of 190 of women with valid pain scores, 103 (52\%) had high pain scores. This group was younger ( 45 yrs. vs. 54 yrs., $p<0.001$ ), more likely to abstain from alcohol (55\% vs. 37\%, $p=0.013$ ), have irritable bowel syndrome (33\% vs. $19 \%, p=0.026)$ and have more pelvic
(1.66 vs. 1.01, $p=0.029$ ) and urologic (0.49 vs. $0.15, p=0.017$ ) surgeries, including surgical menopause $(60 \%$ vs. $30 \%, p$ $=0.004)$. The high pain score group reported less stress incontinence ( $36 \%$ vs. $51 \%, p=0.04$ ), but more sensation of incomplete bladder emptying ( $50 \%$ vs. $23 \%, p=<0.001$ ). The groups did not differ in the incidence of urinary frequency, urgency with leakage or OABq symptom severity score. The high pain score group had a higher Pelvic Organ Prolapse Distress Index (POPDI 6) (9.5 vs. 6.1, $p=>0.001$ ), PFDI 20 summary score (25.8 vs. $17.7, \mathrm{p}=<0.001$ ), a lower (worse) transformed Health Related Ouality of Life score (61.9 v. 72.3, $\mathrm{p}=0.013)$ and were more likely to have a history of anxiety ( $51 \%$ vs. $32 \%, p=0.009$ ) and depression ( $43 \%$ vs. $22 \%, p=0.003$ ). They were also least likely to be given a urologic diagnosis (23\%) versus a gynecologic or pelvic pain diagnosis (31\% and $46 \%$, $\mathrm{p}=<0.001)$.

Conclusions: Women presenting with higher pelvic pain scores are significantly younger, have had more urologic and gynecologic procedures, have more pelvic organ distress and worse quality of life than women with lower pelvic pain scores. They report less irritative voiding symptoms and are less likely to be assigned a urologic diagnosis.

Clinical Relevance: IC and Pelvic Pain-UTI / Inflammatory

\section{Poster \# NM4}

\section{LONG-TERM OUTCOMES FOR 532NM LASER PROSTATECTOMY FOR BPH}

Charles Osterberg, $\mathrm{MD}^{1}$, Ashley Winter, $\mathrm{MD}^{2}$,

Aaron Bernie, $\mathrm{MD}^{2}$, Daniel Lee, $\mathrm{MD}^{2}$ and Benjamin Choi, $\mathrm{MD}^{2}$

${ }^{1}$ Cornell, NY, NY; ${ }^{2}$ Cornell, NY, NY

(Presented by: Charles Osterberg, MD)

Introduction and Objective: Photovaporization of the prostate (PVP) is an effective option for the treatment of lower urinary tract symptoms. However, long-term outcomes have not been closely evaluated.

Methods: Retrospective analysis was performed on 155 consecutive men who underwent PVP using the GreenLight ${ }^{\mathrm{TM}}$ laser system (American Medical Systems, Inc., Minnetonka, MN) with follow-up for at least 24 months. Data was collected on patient demographics, International Prostate Symptom Score (IPSS), maximum flow rate (Qmax), postvoid residual (PVR) and prostate volume were recorded.

Results: Of the 155 patients with follow-up for at least 24 months, 30 men had follow-up for at least 60 months. Of these patients, the median IPSS score was 18, median PVR $103 \mathrm{~mL}$, and median Omax $7 \mathrm{~mL} / \mathrm{s}$. Sixteen percent of the patients were in urinary retention preoperatively, and 33\% had a positive preoperative urine culture. The median energy was $190 \mathrm{~kJ}$ with a median lasing time of 48.6 minutes. Postoperatively, $9 \%$ eventually developed a UTI, with one developing urosepsis that required hospitalization. Two percent developed postoperative urinary retention with a reoperation rate of $1.3 \%$. The median improvement of voiding symptoms (IPSS) was 12 points, with a median improvement of $5 \mathrm{~mL} / \mathrm{s}$ in the Omax and median decrease of $103 \mathrm{~mL}$ in the PVR. of non-obese patients were significantly older (71 vs. 69, $p=0.02$ ), and had lower preoperative Omax rates (7 vs. 8.5, $\mathrm{p}<0.01$ ) than obese patients. For the 30 patients with follow-up for at least 60 months, the median IPSS was 7 (IOR 4-11), median PVR $5 \mathrm{~mL}(5-32)$ and median Omax $10 \mathrm{~mL} / \mathrm{s}(6-15)$ at last follow-up.

Conclusions: PVP is a safe and effective treatment for BPH. The response to treatment is durable, even during long-term follow 
up of more than five years, with relatively low rates of complications.

\section{Clinical Relevance: BPH}

\begin{tabular}{|c|c|}
\hline \multicolumn{2}{|l|}{ Long-term outooness using PVP } \\
\hline \multicolumn{2}{|l|}{ Varitable } \\
\hline N & 155 \\
\hline Modian ags, yours (IQR) & $70(63-75)$ \\
\hline Proopenative urinary refention, $N(\%)$ & $25(16.1 \%)$ \\
\hline Modian IFSS scorc, (IQR) & $18(13-26)$ \\
\hline Modian proopentive Qmax, ml/s (IQRe) & $7(5-10)$ \\
\hline Median preoperative PVR, ml, (IOR) & $103(50-220)$ \\
\hline Median Prostate volume, cm3 (IQR) & $47(30-75)$ \\
\hline \multicolumn{2}{|l|}{ Operative } \\
\hline Median operating room time, min (IQR) & $67.5(60-98)$ \\
\hline Modian lacing time, min (IQR) & 48.6(34.6-68) \\
\hline Enerpy used, lJ (IQR) & $190(130-244)$ \\
\hline Modian Nunber of fibers thed & $1(1-2)$ \\
\hline \multicolumn{2}{|l|}{ Postoperative } \\
\hline Postoperative relention, N(\%) & $3(2.0 \%)$ \\
\hline Reoperation, N(\%) & $2(1.3 \%)$ \\
\hline Postoperative UTi, N(\%) & $14(9.1 \%)$ \\
\hline Median postopentive IPSS, (IQR) & $5(3-9)$ \\
\hline Modian podtoperative Qmax, ml/s (IQR) & $12(8-17)$ \\
\hline Modian postoperative PVR, ml., (IQR) & $5(3-15)$ \\
\hline \multicolumn{2}{|c|}{ Improvement in postoperntive conpared to properative: } \\
\hline $\begin{array}{l}\text { IPSS, Modian (IQR) } \\
\text { Qmax, Median (IOR) }\end{array}$ & $\begin{array}{l}-12(18-6) \\
+5(1-10)\end{array}$ \\
\hline PVR, Madian (IOR) & $-103(208-38)$ \\
\hline
\end{tabular}

\section{Poster \# NM5}

DOES THE INTERSTITIAL CYSTITIS SYMPTOM AND PROBLEM INDEX RELIABLY MEASURE OVERACTIVE BLADDER SYMPTOMS?

Jason Gilleran, $\mathrm{MD}^{1,2}$, Kim A. Killinger, $\mathrm{MSN}^{1}$, Jamie Bartley, $\mathrm{DO}^{1}$, Judith A. Boura, $\mathrm{MS}^{1,2}$ and Kenneth M. Peters, $M^{1,2}$

${ }^{1}$ Beaumont Health System, Royal Oak, MI; ${ }^{2}$ Oakland University William Beaumont School of Medicine, Rochester, MI

(Presented by: Jason Gilleran, MD)

Introduction and Objectives: The validated Interstitial Cystitis Symptom and Problem Index (ICSI-PI) reliably measures symptoms and associated bother in patients with interstitial cystitis. We evaluated whether the ICSI-PI reliably measures overactive symptoms in patients with $\mathrm{OAB}$ with/without incontinence (OAB wet/dry).

Methods: Adults in our prospective observational neuromodulation study were evaluated. Inclusion criteria were $\mathrm{OAB}$ wet/dry and staged lead and generator implant. Subjects completed the ICSI-PI and Overactive Bladder Questionnaireshort form $(\mathrm{OAB}-\mathrm{q})$ at three, six and 12 months post implant. The ICSI-PI is comprised of a symptom index (IC-SI) and bother index (IC-PI), which assess voiding symptoms and pain. The $\mathrm{OAB}-\mathrm{q}$ contains symptom severity (SS) and health related quality of life (HROOL) scales. Descriptive statistics were performed for sample characteristics. At each time point, ICSI and OAB-q SS responses, and IC-PI and OAB-q HROOL responses were compared with Spearman's Correlations in patients that completed both measures.

Results: Of 215 patients (mean age $65.1 \pm 14.2$ years), $80 \%$ were female, $94 \%$ were caucasian, $67.4 \%$ had attended at least some college, $24.5 \%$ had an income $>\$ 70,000 /$ year and $84 \%$ had OAB wet. As shown in the table below, at each time point there was a strong correlation between IC-SI and OAB-q SS scores. Additionally, IC-PI and OAB-q OOL scores were strongly negatively correlated indicating that higher symptom bother negatively impacted HRQOL.

Conclusions: Even though pain is not a component of the OAB syndrome, the ICSI-PI appears to reliably measure voiding symptoms and bother in patients with $\mathrm{OAB}$.
Funding: MPURE (Philanthropy)

Clinical Relevance: LUTS / Voiding Dysfunction

Table. Comparison of Questionnaire Scales.

\begin{tabular}{|l|c|c|c|c|}
\hline & \multicolumn{3}{|c|}{$\begin{array}{c}\text { Symptom Severity } \\
\text { (IC-sI and OAB-q SS) }\end{array}$} & \multicolumn{2}{c|}{$\begin{array}{c}\text { Symptom Bother and HR QOL } \\
\text { (IC PI and OAB-q HRQOL) }\end{array}$} \\
\hline Time P oint & $N$ & $\begin{array}{c}\text { Correlation } \\
\text { Coefficient } \\
0.604\end{array}$ & $N$ & $\begin{array}{c}\text { Correlation } \\
\text { Coefficient } \\
-0.600\end{array}$ \\
\hline 3 months & 190 & 190 & -0.773 \\
\hline 6 months & 129 & 0.748 & 139 & -0.699 \\
\hline 12 months & 113 & 0.793 & 129 & -0.759 \\
\hline
\end{tabular}

\section{Poster \# NM6}

\section{LASERVAPORIZATION OF THE PROSTATE WITH THE 180-W XPS-GREENLIGHT LASER IN PATIENTS WITH ONGOING PLATELET AGGREGATION INHIBITION AND ORAL ANTICOAGULATION}

Malte Rieken, $\mathrm{MD}^{1}$, Daniel Lee, $\mathrm{MD}$, Fujun Zhao, $\mathrm{MD}^{1}$, Heike Pueschel, $\mathbf{R N}^{2}$, Bilal Chughtai, $\mathbf{M D}^{1}$, Steven Kaplan, $\mathbf{M D}^{1}$, Richard Lee, $\mathrm{MD}^{1}$, Alexander Bachmann, $\mathrm{MD}^{2}$ and Alex Te, $\mathrm{MD}^{1}$

${ }^{1}$ Cornell, NY, NY; ${ }^{2}$ University Hospital Basel

(Presented by: Daniel Lee, MD)

Introduction and Objectives: Laservaporization of the Prostate with the Greenlight laser is an established option for the treatment of benign prostate enlargement. The physical properties of the laser make it ideal for the use in patients with increased risk of bleeding. We aimed to characterize the safety and efficacy of the current 180-W XPS-Greenlight laser for the use in patients with ongoing platelet aggregation inhibition and oral anticoagulation.

Methods: Retrospective analysis of data from 401 patients who underwent PVP with the 180-W XPS-laser between 07/ 2010 and 06/2013 at two centers. Intraoperative data, postoperative functional results and postoperative complications were compared between patients with and without use of platelet aggregation inhibition and/or oral anticoagulation (PAI/OC).

Results obtained: Of 401 patients, Aspirin, Clopidogrel and oral anticoagulation were used in 160 (39.9\%), 37 (9.2\%) and 59 (14.7\%) patients, respectively. A single drug, two-drug and three-drug combination was used in 155 (38.7\%), 40 (10.0\%) and $7(1.7 \%)$ of the cases. Patients with the use of PAI/OC were older (74.0 vs. 69.0 years; $p=0.003$ ) than patients not using these drugs, whereas the rate of preoperative retention was comparable (35.1\% vs. $34.7 \%, p=0.92)$. Median lasing time (40.0 vs. $35.0 \mathrm{~min} ; \mathrm{p}=0.11$ ) and number of fibers used (1.0 vs. 1.0; $p=0.49$ ) were comparable between patients using and not using PAI/OC. In contrast, median applied energy was higher in patients using PAI/OC (337 vs. $272 \mathrm{~kJ} ; \mathrm{p}=0.01$ ). Postoperatively, micturition symptoms (IPSS, OoL) and voiding parameters (Omax, postvoid residual volume) improved in both groups of patients. During a maximum follow-up of two years, patients with and without use of PAI/OC did not show any significant differences in the rate of postoperative urinary tract infection $(2.5 \%$ vs. $6.0 \%$; $p=0.08)$, postoperative retention $(6.9 \%$ vs. $4.5 \% ; p=0.30)$, urethral stricture $(1.5 \%$ vs. none, $p=0.09)$ and reoperation $(2.0 \%$ vs. $1.0 \% ; p=0.42$ ).

Conclusions: Laservaporization of the prostate with the 180-W XPS-laser is a safe and effective minimal-invasive treatment option for patients with ongoing platelet aggregation inhibition and/or oral anticoagulation. 
Clinical Relevance: LUTS / Voiding Dysfunction

\begin{tabular}{|c|c|c|c|}
\hline Varisble & $\begin{array}{c}\text { Wilhout anticosgulation/ } \\
\text { autiplatelet }\end{array}$ & $\begin{array}{l}\text { With anticospulation/ } \\
\text { antiplatelet }\end{array}$ & P-value \\
\hline$N(\%)$ & $199(49.6 \%)$ & $202(50,456)$ & \\
\hline Median agc, years & 6 & 74 & $\infty 01$ \\
\hline Preoperative ariusry retention, $N(\boldsymbol{w})$ & $\theta(34.7 \%)$ & $7(35.1 \%)$ & 0.92 \\
\hline Modinn IPSS soore, (CQR) & $21(14-25)$ & $19(14-23)$ & 0.03 \\
\hline $\begin{array}{l}\text { Modian proopenative Qmax, ml/s } \\
\text { (IOR) }\end{array}$ & $79(5.2-10.2)$ & $8.7(5-10.2)$ & 0.93 \\
\hline Modian prooperative PVR, mL (OQR) & $150(69-350)$ & $127.5(80-300)$ & 0.15 \\
\hline \multicolumn{4}{|l|}{ Oxrative } \\
\hline Medien loring time & 35 & 40 & 0.11 \\
\hline Finer [a unod, is & 272 & 337 & 0.01 \\
\hline Number of fibers used & 1.0 & 1.0 & 0,49 \\
\hline \multicolumn{4}{|l|}{ Potoperative } \\
\hline Pondoperative fetertion, $\mathrm{N}(\%)$ & $9(4.5 \%)$ & $14(6.56)$ & 03 \\
\hline Reopenation, $\mathrm{N}(\mathrm{W})$ & $2(1.006)$ & $4(2006)$ & 0.42 \\
\hline Podoperative UTI, N(86) & $12(6.0 \%)$ & $5(25 \%)$ & 0.08 \\
\hline Pootopenative urethnil stricture, $N(\%)$ & $3(1.5 \%)$ & 0 & 0.09 \\
\hline Medim poeteperative IPSS, (MQR) & $9.5(5-17)$ & $8(s-15)$ & 030 \\
\hline $\begin{array}{l}\text { Median postoperntive } \mathrm{Qmax}, \mathrm{m} / \mathrm{s} \\
\text { (IQR) }\end{array}$ & $2.4(6.2-17.8)$ & $11.7(7.1-18.2)$ & 0.34 \\
\hline $\begin{array}{l}\text { Medisan postopentive PVR, mLl, } \\
\text { (GQR) }\end{array}$ & $36(0-9)$ & $41(0-117)$ & 0.21 \\
\hline
\end{tabular}

\section{Poster \# NM7}

\section{THE USE OF THE T1470 SUPERPULSE DIODE LASER IN THE LOWER URINARY TRACT}

Mike Amirian, $\mathrm{MD}$, Alana Murphy, $\mathrm{MD}^{1}$, Patrick Shenot, $\mathrm{MD}^{2}$ and Akhil Das, $\mathbf{M D}^{1}$

${ }^{1}$ TJU, Philadelphia, PA; ${ }^{2}$ TJU, Philadelphia, PA

(Presented by: Mike Amirian, MD)

Introduction and Objectives: Laser technology has changed the endoscopic surgical management of the lower urinary tract. The Diode laser initially used only for the ablation of the prostate, we describe the first experience of the T-1470 Diode laser (Convergence, CA) for enucleation of the prostate and for the treatment of non-malignant lower urinary tract disease.

Methods: 40 patients underwent endoscopic treatment of their lower urinary tract disease with T-1470 Diode laser with an end-firing 600 Micron fiber. Laser energy settings were adjusted to type of procedure. 35 men were treated for Benign Prostatic Hyperplasia (BPH), four men underwent a laser sphincterotomy for Detrusor External Sphincter Dyssynergia (DESD) and two patients were treated for hemorrhagic cystitis. Patients with BPH had urodynamic evidence of Bladder Outlet Obstruction (BOO) prior to treatment. The four patients that had a sphincterotomy had urodynamic evidence of DESD prior to their procedure.

Results: 35 men ages (55-84) had a diode enucleation of the prostate and 29 of these procedures required a transurethral soft-tissue morcellator. In these patients, preoperative IPSS were 21.8 with average peak urinary flow of $4.8 \mathrm{ml} / \mathrm{sec}$. 18/35 of the BPH patients had urinary retention preoperatively. The largest prostate gland treated was 182 grams. Postoperatively at three months, IPSS were 4.7, OOL of 1.6 and peak urinary flow rates of 19.7. Two patients were diagnosed with Gleason $3+3$ adenocarcinoma. Average catheter time was 21.8 hours. Of the four patients in the sphincterotomy group, all were done as an outpatient, none required transfusion and all were with condom catheters within 14 days. The two patients with hemorrhagic cystitis resolved after treatment with 24 hours of catheterization.

Conclusion: Our initial experience with the T-1470 diode laser suggests it is a safe and effective laser for the lower urinary tract. This laser can cut, ablate and coagulate tissue with minimal depth of penetration into tissue. Further studies will help us determine eventual use of this laser in the urinary tract.

Clinical Relevance: LUTS / Voiding Dysfunction

\section{Poster \# NM8}

\section{CHRONIC URINARY SYMPTOMS SECONDARY TO GENITOURINARY TUBERCULOSIS (GU TB) IN PATIENTS IN THE USA: FICTION OR FACT?}

Majid Mirzazadeh, MD and Jacqueline Burnell, BS Wake Forest University School of Medicine, Winston Salem, NC (Presented by: Majid Mirzazadeh, MD)

Introduction: $\mathrm{TB}$ is the second most killing infectious agent worldwide. 9,951 new cases of TB have been reported in the USA in 2012. GU TB consists $5 \%$ of extra pulmonary cases. GU TB, "The Great Imitator," is difficult to diagnose. Most often, it presents with chronic urinary symptoms.

Method: With good knowledge and experience in GU TB presentations and high index of suspicions, we prospectively looked for any possible cases in our patients since 2008. We especially looked for any patient with chronic urinary symptoms of unknown origin. We collected demographic profile, clinical presentations and treatment outcomes of all diagnosed cases.

Results: Seven patients had confirmed diagnosis of GUTB in our center since 2008, including two cases of disseminated military TB from intravesical BCG instillations. In the GUTB group, there were two males and three females, aging from 54 to 72 (mean $62+/-7.8)$. The two males were Hispanic immigrants. The three female patients (Two African American, one Caucasian) were nonimmigrant. The most common symptoms included dysuria (4/5), frequency (4/5), nocturia (3/5), urgency (2/5) and incontinence (2/5). None had generalized TB symptoms. Symptoms started a long time before diagnosis in all patients. Labs showed sterile pyuria (5/5), hematuria (5/5), gross in two and microscopic in three cases and an elevated ESR $(2 / 2)$. Imaging revealed reduced renal function in renogram (4/4), hydronephrosis (4/5), ureteral stricture (4/5), calcification of kidney (4/5), fistula (3/5), decreased bladder capacity (3/5) and renal atrophy $(2 / 5)$. Diagnosis was confirmed with positive urine culture $(4 / 5)$ and pathology $(1 / 5)$. In disseminated BCG infection cases, difficult catheter insertion prior to development of disseminated TB was the highlight of history in both. Symptoms included fever, night sweats, cough and weight loss in both patients. Lower urinary tract symptoms were also present in both cases. CXR demonstrated miliary TB in both. Both patients were treated without any sequalae.

Conclusion: GU TB still exists in US patients with any ethnicity, with or without immigration history. Patients usually present with long standing urinary symptoms. With nonspecific symptoms, a high index of suspicion and experience is required for timely diagnosis and management. Complications may persist even with proper treatment. To prevent disseminated BCG infection, any kind of difficult catheterization should preclude from intravesicular BCG installation.

\section{Poster \# NM9}

OUALITY OF LIFE IN NEUROGENIC BLADDER PATIENTS AFTER SUPRAPUBIC TUBE PLACEMENT.

Rebecca S. Lavelle, MD, Chasta Bacsu, MD, Louise A. Gliga, BS and Gary E. Lemack, MD

University of Texas Southwestern Medical Center, Dallas, Texas (Presented by: Rebecca S. Lavelle, MD) 
Introduction and Objectives: Suprapubic catheterization (SPC) can be an effective means of managing complex patients with neurogenic bladder (NGB) conditions in which other options are unfeasible or impractical. SPC is often chosen by the patient and the clinician to improve effective bladder emptying and quality of life (OoL). This study aims to evaluate patient satisfaction in patients with NGB who have elected to undergo SPC, as well as assess adverse events (AEs) related to the procedure.

Methods: This is a retrospective chart review utilizing data from an IRB approved database of patients with a variety of NGB conditions followed by a single surgeon from $1 / 1 / 2003$ to $6 / 30 / 2013$. Patients who underwent SPC placement (all done under anesthesia) were invited to complete a validated, single item Patient Global Impression of Improvement (PGI-I) questionnaire. Success or positive response was defined as 1 or 2 on a scale of 1-7. Demographic information and AEs (Clavien classification IIIb or greater) were collected from the database. Patients were excluded from questionnaire mailing if they were not English speaking or deceased, though were included in assessment of adverse events.

Results: Of the 128 patients who underwent SPC, 105 patients (64 female, 41 male) fit the inclusion criteria and were sent the PGI-I questionnaire. Response rate was 44.8\% (47/105). Mean age at the time of SPC placement was 54.9 years. Mean follow up was 25.1 months (1-120). Overall, 53 pts had MS, 26 had SCI, four had PD and 22 had other neurologic disorders (cerebrovascular accident, cerebral palsy, brain tumor, seizure disorder, transverse myelitis). Overall, success was seen in $41 / 47$ patients (87.2\%). Only 6.4\% (3/47) patients reported a negative PGI-I (score 5-7/7). An early postoperative complication (within 30 days of surgery) was seen in 1/128 patients $(0.08 \%)$ and consisted of bowel perforation requiring small bowel resection.

Conclusions: SPC is an effective solution in many carefully selected patients with NGB conditions. Over $85 \%$ considered the SPC to have improved their urological quality of life with a mean follow-up of over two years. Adverse events are rare, though can be particularly serious in this group of neurologically impaired patients.

Financial Funding: Departmental

\section{Poster \# NM10}

\section{CHARACTERIZATION OF MULTIPLE SCLEROSIS PATIENTS BASED ON THE ACTIONABLE BLADDER SYMPTOM AND SCREENING TOOL (ABSST)}

Areeba Sadiq, $\mathbf{M S}^{1}$, Margarita Aponte, $\mathbf{M D}^{1}$, Ilya Kister, $\mathbf{M D}^{2}$, Carrie Sammarco ${ }^{2}$, Victor Nitti, MD $^{1}$ and Benjamin Brucker, $\mathrm{MD}^{1}$

${ }^{1}$ Department of Urology, New York University Langone Medical Center, New York, NY; ${ }^{2}$ Neurology, New York University Langone Medical Center, New York, NY

(Presented by: Margarita Aponte, MD)

Introduction: The Actionable Bladder Symptom and Screening Tool (ABSST) is a validated screening tool to be used in patients with multiple sclerosis (MS) to screen for Neurogenic overactive bladder symptoms (NDO) that are amenable to treatment. This simple 8-item questionnaire aids in identifying patients that need a referral to a urologist. Although up to $80 \%$ of patients diagnosed with MS experience these symptoms, evaluation and treatment are significantly under accessed. The objective of this study is to characteristics a sample of MS patients that would be recommended for urologic evaluation based on the ABSST.

Methods: This was a prospective observational study. One hundred patients diagnosed with MS, but currently not seeing a urologist, were enrolled from an MS Center. Patients completed the ABSST short form and general demographic questions. An ABSST score $\geq 3$ met criteria for recommending further urologic evaluation. X2tests were used for comparison of categorical variables.

Results: Patient's mean age was 44.5 years and average time since diagnosis was 10.4 years. There were 79 women and 21 men. Ethnicities included 45\% Caucasian, 21\% African American, 21\% Hispanic, 2\% Asian and 11\% Other/Multiracial. 27 patients had an ABSST score $>3$. When compared to patients with an ABSST $<3$, those with an ABSST $>3$ had a significant difference in level of education ( $p<0.05)$, type of mobility used $(p<0.05)$ and employment status $(p=0.005)$. See Figure 1. Patients with an ABSST $>3$ were more likely to have seen a doctor in the past for bladder problems ( $52 \%$ vs. $27 \%$; $p=0.019$ ), more frequently for urinary incontinence (19\%) and urinary tract infections (13\%). They were also more likely to be using medications for bladder symptoms ( $23 \%$ vs. $4 \%$; $p=0.004)$ or to have used them in the past ( $42 \%$ vs. $15 \%$; $p=0.004$ ).

Conclusions: The differences observed in MS patients who score positive on the ABSST may represent the progressive nature of the disease and its association with NDO. These findings highlight the importance of continued screening for NDO in patients with MS. The ABSST is a valuable simple tool for providers to efficiently identify and refer MS patients with urological symptoms for further evaluation and treatment.

Clinical Relevance: Neurogenic Bladder

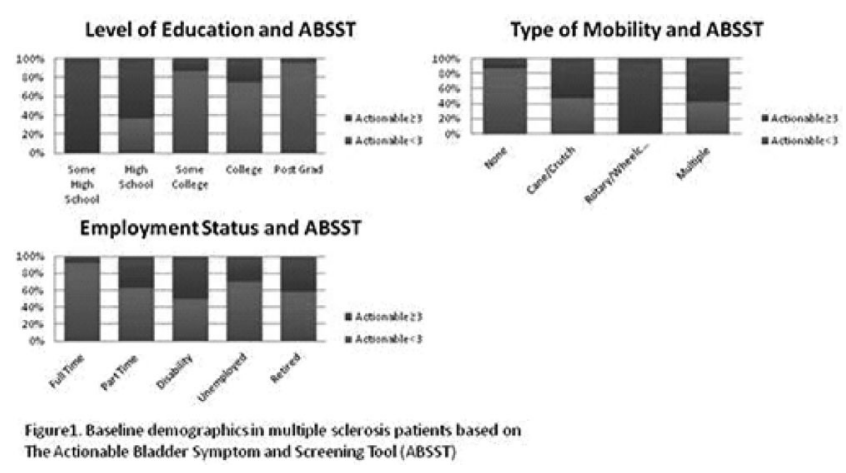

\section{Poster \# NM11}

ACTIONABLE: A SIMPLE AND EFFECTIVE WAY OF ASSESSING LOWER URINARY TRACT DYSFUNCTION IN PATIENTS WITH MULTIPLE SCLEROSIS

Margarita Aponte, $\mathrm{MD}^{1}$, Areeba Sadiq, $\mathrm{MS}^{1}$, Jennifer Kalina ${ }^{2}$, Nirit Rosenblum, $M^{1}$, Victor Nitti, $M^{1}$

and Benjamin Brucker, $M^{1}$

${ }^{1}$ Department of Urology, New York University Langone Medical Center, New York, NY; ${ }^{2}$ Department of Neurology, New York

University Langone Medical Center, New York, NY

(Presented by: Margarita Aponte, MD)

Introduction and Objectives: The Actionable Bladder Symptom and Screening Tool (ABSST) is a validated tool used in patients with multiple sclerosis (MS) to screen for Neurogenic overactive bladder symptoms (NDO) in need of urologic referral. The objective of this study is to correlate the ABSST with several questionnaires including the Overactive Bladder 
Questionnaire (OABq), Patient Global Impression of Severity (PGI-S), International Consultation on Incontinence Questionnaire (ICIO), and Medical Epidemiology and Social Aspects of Aging (MESA).

Methods: This was a prospective observational study. 100 patients diagnosed with MS, not currently seeing a urologist, were enrolled from an MS center. Patients filled out a validated short form of the ABSST, the OABq short form, the PGI-S, the ICIO and the MESA. An ABSST score $>3$ met criteria for urologic referral and was considered a positive screening test. OAB subscale scores grouped responses related to symptom bother and Ouality of life (HROL), which was further subdivided into HROL-Coping, HROL-Sleep or HROL-Emotions. A high score for the symptoms subscale indicated worse symptoms and a low score of the quality of life subscales indicated worse quality of life. The MESA scores grouped responses that characterized either urge or stress incontinence. There were no subscales for PGIS or ICIO. Mean questionnaire scores were compared between patients who screened positive or negative for the ABSST using one-way ANOVA and X2 tests.

Results: Of 100 patients, 27 patients had a positive ABSST. Patients with a positive ABSST had a significant difference in the mean scores of the following subscales: OABq Symptoms (57.4 vs. $15.0 ; p=0.000)$; OAB-Total HRQL (50.4 vs. 89.9; $\mathrm{p}=0.000)$; OAB-HRQL Cope (43.4 vs. 89.4; $\mathrm{p}=0.000)$; OABHROL Sleep (42.5 vs. 84.2; $\mathrm{p}=0.000$ ); OAB-HRQL Emotion (62.1 vs. 93.7; $p=0.000$ ); MESA-Urge Incontinence (40.4 vs. 12.3; $\mathrm{p}=0.000)$; MESA-Stress Incontinence (33.8 vs.15.0; $\mathrm{p}=0.000$ ); and ICIO-SF (8.2 vs. 2.3; $p=0.000)$. There was a significant relationship between PGIS Score and a positive ABSST ( $p$ $=0.000$ ).

Conclusions: The ABSST is a valuable simple tool for identifying patients in need of further urological evaluation. A positive screening response on the ABSST correlates and captures the severity of symptoms, impact on quality of life and classifications of both urge or stress incontinence across several overactive bladder and urinary incontinence questionnaires.

Clinical Relevance: Neurogenic Bladder

\section{Poster \# NM12}

\section{LOWER URINARY TRACT PHENOTYPE IN MEN AND WOMEN} WITH MULTIPLE SCLEROSIS

Ariana Smith, $\mathrm{MD}^{1}$, Tom Bavaria, BS ${ }^{1}$, Mary Wang, MSN, CRNP ${ }^{1}$, Diane Newman, DNP, CRNP ${ }^{1}$, Michael Ruggieri, $\mathrm{PhD}^{2}$ and Malykhina Anna, $\mathrm{PhD}^{1}$

${ }^{1}$ Division of Urology, University of Pennsylvania, Philadelphia, PA; ${ }^{2}$ Division of Anatomy and Cell Biology, Temple University, Philadelphia, PA

(Presented by: Ariana Smith, MD)

Introduction: Lower urinary tract symptoms (LUTS) are prevalent among men and women with multiple sclerosis (MS). Symptoms may be obstructive (hesitancy, weak/interrupted stream, incomplete emptying, retention), overactive (frequency, urgency, urgency incontinence, nocturia) or both. Effective treatment paradigms are lacking in this complex population. Phenotyping may provide insight into pathophysiology of LUT disease in MS, disease progression, novel therapeutics and response to therapy.

Methods: Patients with MS and LUTS were recruited from urology and neurology. Participants were asked to complete several measures assessing demographics, physical function and voiding and bowel function (Urinary Distress Inventory, American Urological Association Symptom Score (AUASS),
MESA Questionnaire, Short Form Health Survey (SF-36), International Consultation on Incontinence Questionnaire Bowel, Expanded Disability Status Scale and Functional System Scale). Data was managed using REDCap.

Results: 20 men and women with MS and LUTS were included in the pilot analysis. Mean age was 46 years with $80 \%$ Caucasian and 20\% African American. Mean time since MS diagnosis was 13.1 years and mean time since LUTS began was 8.8 years. $20 \%$ experienced LUTS prior to MS diagnosis or symptoms. $60 \%$ described their initial bladder symptom as overactive and $40 \%$ as obstructive. $75 \%$ experienced urinary frequency, urgency and urgency incontinence and 80\% nocturia. 50\% endorsed difficulty voiding and incomplete emptying. $90 \%$ reported some urinary leakage. Average AUASS was 17 and $80 \%$ were dissatisfied. Bowel function was reported as difficult, requiring straining in $60 \%$ and requiring digital manipulation in $25 \%$. General health was reported as very good in $40 \%$ and good/fair in $60 \%$. $75 \%$ were unable to participate in moderate activity, $60 \%$ were unable to climb one flight of stairs and $70 \%$ were unable to bathe and dress themselves.

Conclusions: Overactive and obstructive LUTS were common among patients with MS. LUTS began on average 4.3 years after MS diagnosis with 20\% reporting LUTS as their initial presentation. LUTS did not discreetly fall into obstructive or overactive categories but rather fell on a spectrum with varying degrees of each. Patients with greater physical function impairment appeared to suffer from greater LUTS and bowel dysfunction. $80 \%$ of patients reported dissatisfaction with voiding symptoms. Further study in this complex population is needed.

Funding: P20 DK097819-01

Clinical Relevance: LUTS / Voiding Dysfunction

\section{Poster \# NM13}

\section{PREDICTING WHICH PATIENTS WILL ONLY UNDERGO A} SINGLE ONABOTULINUMTOXINA INJECTION: RESULTS FROM A TERTIARY REFERRAL CENTER.

Rebecca S. Lavelle, MD, Burhan Coskun, MD, and Gary E. Lemack, MD

University of Texas Southwestern Medical Center, Dallas, Texas (Presented by: Rebecca S. Lavelle, MD)

Introduction and Objectives: OnabotulinumtoxinA (BTX) detrusor injection is an effective treatment for patients with neurogenic detrusor overactivity (NDO) and overactive bladder (OAB). Still, not all patients request repeat injections. We sought to identify patient characteristics associated with the decision to not request repeat BTX injections.

Methods: We analyzed demographic (including questionnaire) and urodynamic (UDS) data from an IRB approved database of patients with neurogenic bladder conditions and OAB. Patients were excluded for: prior inclusion in a BTX trial $(n=6)$, deceased from unrelated causes $(n=2)$, initial injection within the last 9 months $(\mathrm{n}=27)$.

Results: From January 2009 through August 2013, 109 unique patients underwent a total of 206 injections. All patients were invited to call for repeat injection any time three months after the original injection. Of the 74 patients (59 NDO, $15 \mathrm{OAB}$ ) who met inclusion criteria, 56 (76\%) underwent or have scheduled repeat injections (RI) and 18 (24\%) had a single injection only (SI). In the SI group, the indications for injection were $\mathrm{OAB}$ (44.4\%) and NDO (55.6\%), and the corresponding values in the RI group were OAB (12.5\%) and NDO (87.5\%), $(p=0.0065)$. Of the SI patients with $\mathrm{OAB}$, four of eight patients had secondary 
diagnoses: three with prior prostate cancer therapy, one with Painful Bladder Syndrome (PBS). Of the RI patients with OAB, three of seven had secondary diagnoses (prior urologic surgery or pelvic radiation). The most common reasons for a SI were insufficient response (66.7\%) or dissatisfaction due to de novo clean intermittent catheterization (CIC) (16.7\%). Of the 12 with insufficient response, six required other intervention (catheterization, augmentation, cystectomy). De novo CIC was required in $27.8 \%$ of those in the SI group and $3.6 \%$ in the RI group $(p=0.0079)$. Baseline UDS and questionnaire data, shown in Table 1, did not differ between groups.

Conclusions: In our experience, patients with refractory complex forms of OAB were more likely to have a SI only. Insufficient response and de novo CIC were the most common reasons for SI. Neither UDS nor questionnaire data were predictive of a single BTX injection.

Financial Funding: None

Clinical Relevance: LUTS / Voiding Dysfunction

Table 1: Patient Characteristics and Urodynamic Data in BTX Single Injection (SI) vs. Repeat Injection (RI)

\begin{tabular}{|l|l|l|l|}
\hline & $\begin{array}{l}\mathrm{SI} \\
(\mathbf{n}=18)\end{array}$ & $\begin{array}{l}\mathrm{RI} \\
(\mathbf{n}=56)\end{array}$ & P value \\
\hline Age, yrs, mean \pm SD & $56.5 \pm 14$ & $59.9 \pm 18.5$ & 0.42 \\
\hline Gender & & & 0.82 \\
\hline Male, $\mathbf{n}(\%)$ & $5(27.8 \%)$ & $15(26.8 \%)$ & \\
\hline Female, $\mathbf{n}(\%)$ & $13(72.2 \%)$ & $41(73.2 \%)$ & \\
\hline Diagnosis & & & 0.0065 \\
\hline NDO, $\mathbf{n}(\%)$ & $10(55.6 \%)$ & $49(87.5 \%)$ & \\
\hline OAB, $\mathbf{n}(\%)$ & $8(44.4 \%)$ & $7(12.5 \%)$ & \\
\hline De novo CIC, $\mathbf{n}(\%)$ & $5(27.8 \%)$ & $2(3.6 \%)$ & 0.0079 \\
\hline$\%$ Detrusor Overactivity & $68.8 \%$ & $77.3 \%$ & 0.52 \\
\hline MCC, mL, mean \pm SD & $215 \pm 173.8$ & $238.5 \pm 162.9$ & 0.76 \\
\hline Reflex volume, mL, mean \pm SD & $136.7 \pm 96.7$ & $175 \pm 160.6$ & 0.77 \\
\hline pdetMax, cmH2O, mean \pm SD & $57.5 \pm 28.5$ & $52 \pm 35.9$ & 0.68 \\
\hline OABq-SF, Score (6-36), mean \pm SD & $21.1 \pm 9.8$ & $24.9 \pm 8.4$ & 0.25 \\
\hline PGIS, Score (1-4), mean \pm SD & $3.6 \pm 0.7$ & $3.5 \pm 0.7$ & 0.45 \\
\hline
\end{tabular}

\section{Poster \# NM14}

\section{INCIDENCE OF ADVERSE EVENTS AFTER GREATER THAN 360 CUMULATIVE UNITS OF BOTULINUM TOXIN GIVEN WITHIN A THREE MONTH TIME PERIOD}

Nitya Abraham, $M D$, Umaphorn Nuanthaisong, $M^{1}$ and Howard Goldman, $\mathbf{M D}^{2}$

${ }^{1}$ Cleveland Clinic, Cleveland, Ohio, Navamindradhiraj University, Bangkok, Thailand; ' ${ }^{2}$ Cleveland Clinic, Cleveland, Ohio

(Presented by: Nitya Abraham, MD)

Introduction and Objective: The manufacturer of Botox recommends no more than 360 cumulative units within a three month interval. Higher doses of Botox within three months can result in spread of toxin effect, potentially causing dysphagia and paralysis of respiratory muscles. The aim of this study was to determine the occurrence of all adverse events, especially life-threatening adverse events, after the injection of a cumulative dose of more than 360 units Botox within a three month interval.

Methods: This is a retrospective cohort study of patients who received more than 360 units Botox within a three month interval, with at least one urologic indication for injection between $1 / 1 / 2002$ to $1 / 1 / 2013$. The rate of any adverse event up to eight days post-injection, and life-threatening adverse events up to 90 days post-injection was compared between injection sessions meeting the dosage guidelines and injection sessions exceeding the dosage guidelines.

Results: 13 patients met study criteria. There were no adverse events after injection sessions falling within manufacturer recommended dosage guidelines. There were six adverse events out of a total of 135 (4.4\%) injection sessions that exceeded manufacturer recommended dosage guidelines. These adverse events were minor and eventually resolved. There were no life-threatening adverse events in either group. See Table 1.

Conclusion: High cumulative doses of Botox were tolerated in a retrospective cohort of patients undergoing injection for multiple indications. There were no life-threatening adverse events. Prospective studies are necessary to redefine the maximum cumulative dosage of Botox that can be safely administered within a certain time period.

Funding: None

Clinical Relevance: Neuromodulation

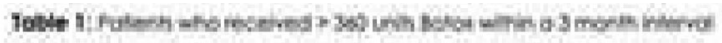

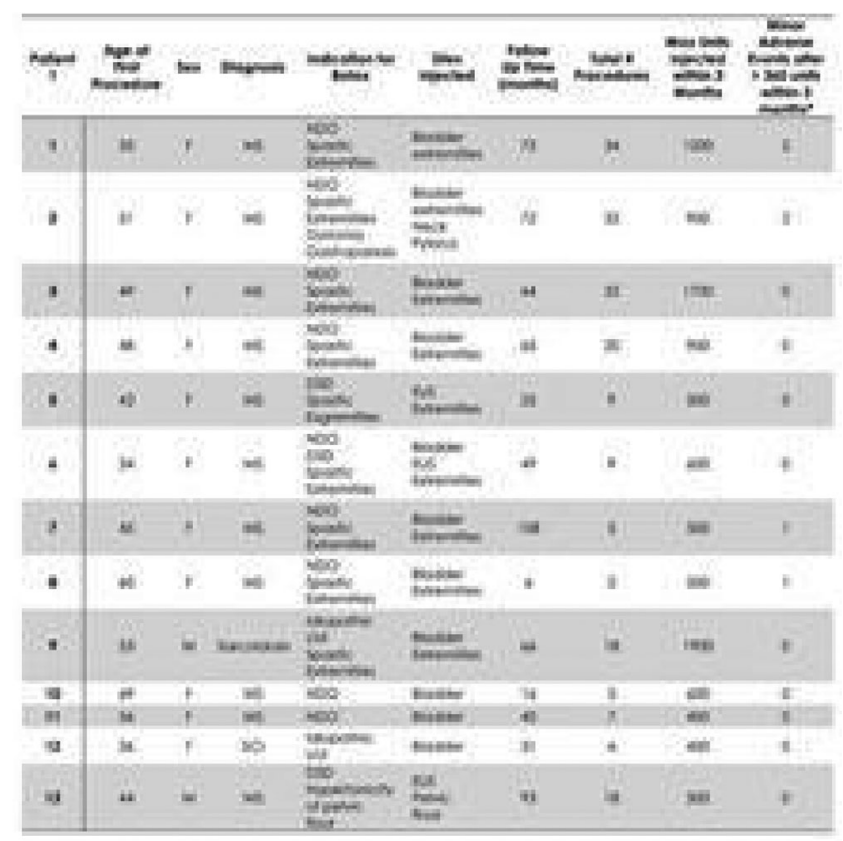

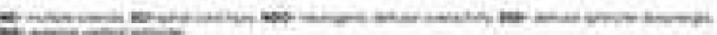

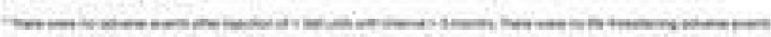

\section{Poster \# NM15}

BOTULINUM TOXIN A: THE SHIFT TO A MINIMALLY INVASIVE MANAGEMENT OF NEUROGENIC BLADDERS

Michael Aberger, MD, Bradley Wilson, MD, Arther Andrew, MD and Priya Padmanabhan, MD, MPH University of Kansas

(Presented by: Michael Aberger, MD)

Introduction: The key in management of neurogenic dysfunction of the lower urinary tract is the maintenance of low storage pressures. Augmentation cystoplasty (AC) has traditionally been used for creation of a high capacity, low-pressure reservoir. The FDA approved Botulinum toxin A (BTX-A) in August of 2011 for the treatment of neurogenic detrusor over activity (NDO). Multiple studies demonstrate BTX-A effectiveness in neurogenic bladders with significant improvements in incontinence episodes and urodynamic parameters. Our study reviews how the advent of BTX-A has changed modern treatment of NDO. 
Methods: We performed a retrospective review from 20032013 of patients seen in our urology practice with anticholinergic refractory NDO that underwent AC or cystectomy with urinary diversion. We analyzed their charts and testing data to see how many patients would have met the indication for BTXA. Furthermore, we compared our practice since the incorporation of BTX-A to see how our treatment of NDO has changed. Results: 59 patients met inclusion criteria for our study. 34 patients underwent open reconstructive surgery for NDO. The remaining 25 patients have been managed with BTX-A detrusor injections. Review of the 34 patients that underwent open surgery revealed 21 (62\%) would have met indication for BTX-A injections. Additionally, 30 of the 34 patients that underwent reconstructive surgery had surgery within the first eight years of the study resulting in nearly four open surgeries for NDO per year. After the FDA approval of BTX-A 2011 for NDO, the number of open reconstructive surgeries at our institution reduced to less than two per year. During that same time period an average of 12 patients per year were managed with BTX-A. Of the 34 reconstructive patients, $14(41 \%)$ had a complication with 10 (29\%) requiring a repeat procedure. Conversely, only one BTX-A patient (4\%) had a complication for which further intervention was required.

Conclusions: Anticholinergic refractory NDO is a complex disorder that impairs quality of life and can threaten the upper urinary tracts. BTX-A has become an effective minimally invasive alternative to urinary reconstruction. This study demonstrates how the addition of BTX-A has positively evolved the care of NDO patients.

Clinical Relevance: Neurogenic Bladder

\section{Poster \# NM16}

\section{COMPLETELY INTRACORPOREAL ROBOTIC-ASSISTED LAPAROSCOPIC ILEOVESICOSTOMY IS ASSOCIATED WITH EARLIER RETURN OF BOWEL FUNCTION}

MaryEllen T Dolat, MD, Joseph R. Habibi, MD, Greg Wade, MD, Blake W. Moore, MD, Lance J. Hampton, MD

and Adam P. Klausner, MD

Virginia Commonwealth University, Richmond, VA

(Presented by: MaryEllen T Dolat, MD)

Objectives: Ileovesicostomy has been used as a treatment for neurogenic bladder dysfunction in individuals unwilling or unable to perform intermittent catheterization. The open technique is associated with post-operative complications including wound infection, urethral incontinence and extended length of hospital stay (LOS). Using the technique of intracorporeal laparoscopic bowel-to-bowel anastomosis may improve post-operative recovery and complications. However, there is sparse literature describing this technique. Our goal is to report initial results for intracorporeal laparoscopic bowelto-bowel anastomosis robotic-assisted as compared to open ileovesicostomy.

Methods: A retrospective review of fifteen open and four robotic ileovesicostomy procedures performed between September 2005 and April 2011 was performed. Demographic data, intraoperative/postoperative data and follow up data were obtained. Intraoperative/postoperative parameters consisted of operative time, estimated blood loss (EBL), complications, return of bowel function and LOS. Follow up data included continence, urodynamic studies and length of follow up.

Results: A total 19 ileovesicostomies were performed (15 open, four robotic). Age, BMI and pre-operative urodynamics were similar between groups. Bowel function returned earlier in the robotic group ( 9 days vs. 3.8 days, $p=0.02$ ). However, operative time (252 min vs. $290 \mathrm{~min}, \mathrm{p}=0.28$ ), estimated blood loss ( $218 \mathrm{~mL}$ vs. $131 \mathrm{~mL}, \mathrm{p}=0.15$ ), LOS ( 16 vs. 8 days, $\mathrm{p}=0.30$ ) and follow-up (38 months vs. 26 months, $\mathrm{p}=0.16$ ) were similar between open vs. robotic groups, respectively. There were no major intraoperative complications in either group. In the robotic group, one female patient with known urethral erosion had continued urethral incontinence requiring periurethral bulking and subsequent sub-urethral sling with resolution of symptoms. One male patient had elevated residual volumes $(300 \mathrm{ml})$ and cystoscopic evaluation demonstrated a small caliber vesicostomy. Due to the patient's concern about potential infection, an open conversion to ileal conduit was performed 16 months after his initial surgery.

Conclusion: The outcomes for open and robotic-assisted ileovesicostomies performed were similar. However, completely intracorporeal robotic-assisted laparoscopic ileovesicostomy may be associated with earlier return of bowel function.

Clinical Relevance: Neurogenic Bladder

\section{Poster \# NM17}

\section{SEX DIFFERENCES IN URINARY SYMPTOMS IN MULTIPLE SCLEROSIS}

John Stoffel, MD, Daniela Wittman, LMSW, Joseph Papin, BS, Anne Cameron, MD, Ouentin Clemens, MD, Chang He, PhD, John Wei, MD and Aruna Sarma, PhD

University of Michigan, Ann Arbor, MI

(Presented by: John Stoffel, MD)

Introduction: Studies have demonstrated sex differences in disease progression among multiple sclerosis (MS) patients. We reviewed data from multiple sclerosis patients (MS) to identify sex differences in prevalence of urinary complaints, impact of symptoms and bladder physiology.

Methods: We conducted a retrospective chart review of men and women with multiple sclerosis and refractory urinary symptoms who underwent urodynamic testing (UDS) at a referral neuro-urology clinic over a five year timeframe (20072012). Type and impact of urinary symptoms were assessed at index urodynamic visit through review of chief complaints, the AUA Symptom Index, the Incontinence Severity Index (ISI) and urodynamic studies. Urodynamic findings of detrusor overactivity (DO), detrusor external dyssynergia (DSD) and low compliance $(<20 \mathrm{cc} / \mathrm{cm} \mathrm{H20})$ were considered dichotomous variables. We tested for associations between sex and urinary variables (presenting chief complaint, UDS findings, post void residual, AUA/ISI symptom scores).

Results: Clinical and UDS data from 118 unique MS patients (35 male, 83 female) were reviewed. Both groups were similar in age, BMI, length of time with MS, prevalence of chronic urinary tract infections, use of intermittent catheterization and indwelling catheters. More men (27\%) reported obstructive symptoms as the most bothersome urinary problem, compared to women $(10 \%)(p=0.05)$ although urinary frequency/urgency was the most common most bothersome complaint overall (men 62\%, women 71\%, $\mathrm{p}=0.30$ ). Both groups reported similar quality of life impact from urinary symptoms based on total AUA (male 19, female 17, $\mathrm{p}=0.44$ ), bother $(4.1,3.9, \mathrm{p}=0.77$ ), and ISI $(14,18, p=0.10)$ scores. On urodynamic studies, men had significantly higher voiding pressures compared to women

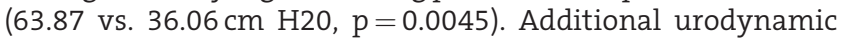
findings are summarized in Table 1. 
Conclusions: In this cohort of MS patients, both men and women were impacted by urinary symptoms but a higher percentage of men reported obstructive symptoms as the most bothersome complaint. Men also had higher voiding pressures on urodynamic testing.

Clinical Relevance: Neurogenic Bladder

\begin{tabular}{|c|c|c|c|}
\hline & Male $(n=35)$ & Female $(n=83)$ & P \\
\hline PVR before study (mean $\leftleftarrows s e)$ & $153.00 \pm 19.90$ & $125.30 \pm 15.96$ & 0.31 \\
\hline Capasity (mean $\neq$ re) & $299.83 \div 23.15$ & $277.16 \pm 16.84$ & 0.41 \\
\hline Low Compliance $(n, k)$ & $4(11.43 \%)$ & $7(8.43 \%)$ & 0.73 \\
\hline With $D O(n, 96)$ & $20[57.14 \%)$ & $44[53.01 \%]$ & 0.68 \\
\hline With DSD $(n, \%)$ & $10(28.57 \% 6)$ & $18(21.69 \%)$ & 0.42 \\
\hline With Atonic Bladder $(n, 36)$ & $7(20.00 \%)$ & $25(30.49 \%)$ & 0.24 \\
\hline With inconthence (sUl of urge) $(\mathrm{n}, 4$ ) & $6(18.1886)$ & 28 (33.73\%) & 0.10 \\
\hline Pdet (mean \pm se) & $63.87 \pm 7.66(n=23)$ & $36.06 \pm 5.60(n=42)$ & $0.0045^{\circ}$ \\
\hline
\end{tabular}

\section{Poster \# NM18}

CHANGES IN BLADDER MANAGEMENT IN PATIENTS WITH NEUROGENIC BLADDER WITH FDA APPROVAL OF ONABOTULINUMTOXINA: A RETROSPECTIVE REVIEW

Goldmark Ellen, MD, Benjamin Yuh, MD

and Ginsberg David, MD

USC, Los Angeles, CA

(Presented by: Goldmark Ellen, MD)

Introduction and Objective: Patients with NGB can present with a variety of urologic manifestations, often requiring complex surgical reconstruction. The FDA approval of onabotulinumtoxinA (BoNT-A) in August of 2011 provided an alternative, less-invasive option for patients with neurogenic detrusor overactivity (NDO) that failed more conservative therapy. We hypothesized the use of BoNT-A would alter the number and type of procedures performed.

Methods: We retrospectively reviewed a single-surgeon's experience with NGB patients at a large rehabilitation hospital. Two similar eight month time periods were compared: one prior to and one following the FDA approval of BoNT-A for NDO in August 2011. Data obtained included demographics, urodynamic outcomes and treatments. Outside of clinical trials, BoNT-A was not used for NGB at our institution prior to its FDA approval.

Results: During the eight months prior to the approval of BoNTA (January - August 2011), 18 patients (12 males, six females, mean age 28.6) underwent a total of 25 reconstructive procedures (see table). Neurologic conditions were: 14 SCI (4 cervical, 10 thoracic) and one each- transverse myelitis, multiple sclerosis (MS), cerebral palsy and spina bifida (SB). Seventeen months after FDA approval of BoNT-A (January - August 2013) seven patients (five males, two females, mean age 39.6) underwent a total of 10 surgeries (see table). Neurologic conditions were: three thoracic SCI, one Arnold Chiari, two MS and one SB. As noted in the table, a significant decrease in the number of bladder augmentations and abdominal stomas were found post-BoNT-A approval. In addition, a greater percentage of the procedure done post-BoNT-A approval were for a poor outlet $(5 / 10=50 \%)$ compared to pre-approval $(8 / 25=32 \%)$.
Conclusion: Introduction of BoNT-A for the treatment of patients with NDO has and will continue to decrease the number of invasive bladder reconstructive surgeries done in patients with NGB. Procedures needed to address a suboptimal outlet and construct an abdominal stoma should not be impacted by BoNT-A. Bladder augmentation will likely be reserved for patients who continue to have urinary incontinence and/or poor compliance after failed BoNT-A treatment. Clinical relevance: Neurogenic Bladder

\begin{tabular}{|l|c|c|}
\hline Procedures & Pre-Approval & Post-Approval \\
\hline $\begin{array}{l}\text { Total Lower Urinary Tract } \\
\text { Reconstructive Procedures }\end{array}$ & 25 & 10 \\
\hline Augment & 9 & 2 \\
\hline Abdominal stoma & 7 & 2 \\
\hline Outlet enhancing procedures & 8 & 5 \\
\hline Cystectomy & 1 & 1 \\
\hline BoNT-A injection & 0 & 16 \\
\hline
\end{tabular}

\section{Poster \# NM19}

\section{FISTULA CARE USING A COLLABORATIVE APPROACH IN SOUTHWESTERN UGANDA}

Erin Mellano, $\mathrm{MD}^{1}$, Musa Kayondo, $\mathrm{MD}^{2}$, Tamara Grisales, $\mathbf{M D}^{1}$, Amy Stenson, $\mathbf{M D}^{3}$, Una Lee, $\mathbf{M D}^{4}$, Michael Margolis, $\mathbf{M D}^{1}$ and Christopher Tarnay, $\mathbf{M D}^{1}$

${ }^{1}$ University of California, Los Angeles (UCLA) David Geffen School of Medicine, Los Angeles, CA; ${ }^{2}$ Mbarara University of Science and Technology (MUST), Mbarara, Uganda; ${ }^{3}$ Oregon Health and Science University, Portland, OR; ${ }^{4}$ Virginia Mason Medical Center, Seattle, WA

(Presented by: Erin Mellano, MD)

Introduction and Objective: Our objective is to report on the results of a collaborative fistula care program between physicians from the United States and from Southwestern Uganda. Fistulae from traumatic obstetrical injuries in developing countries are a global health problem due to a lack of access to health care. An estimated 5\% of Ugandan women suffer from obstetrical fistulae, with 1,900 new cases each year. In 2007, a non-profit organization, Medicine for Humanity, partnered with physicians at UCLA and with those at Mbarara University of Science and Technology (MUST) Hospital in Uganda to establish a sustainable surgical training and educational fistula care program.

Methods: A retrospective review of the hospital charts of patients treated at twice-annual surgical fistula camps. Treatment teams consisted of UCLA and MUST surgeons, fellows, residents, anesthesiologists, nurses and educators. Data was available between March 2010 and September 2013. Data abstracted included demographics, operative reports and postoperative course.

Results: Two-hundred and eighteen women underwent surgery for complex pelvic floor disorders. 125 of these women underwent surgery for a genitourinary fistula alone. Of these 125 women, four were lost to follow-up and 20 recently had surgery within the last month and are not included. Mean age was 31.7 years (15-70 SD $+/-12$ years). Average distance traveled to MUST was $47.5 \mathrm{~km}$ (1-310 km). Mean parity was 3.1 (1-12 SD + /-2.6). Peripartum risk factors were: obstructed labor $83 \%$ (115/138), cesarean section 60\% (82/138) and fetal stillbirth 71\% (98/138). Prior to presentation, mean duration of incontinence was 6.1 years (1 month- 45 years). Results from the 101 patients with follow-up data after surgical fistula repair using the Waaldjik classification system were: 46 vesicovaginal (type I), 48 urethrovaginal (24 type IIA, 24 type 
IIB), two ureterovaginal and five patients with complex fistulae. A total of 93/101 (92\%) women were dry from the fistula after surgery. 61 patients were dry after one surgery and 32 were dry after more than one repair. Eight patients were not cured.

Conclusions: We present the results from three years of a joint program to treat urogenital fistulae. Fistula cure rates of over $90 \%$ demonstrate effective surgical correction of a dramatically undertreated condition through collaborative efforts. Continued focus on training local physicians and medical educators to raise awareness and to battle this epidemic are essential.

Clinical Relevance: Reconstruction

\section{Poster \# NM20}

\section{SEEKING THE TRUTH ABOUT PRIMARY ELECTIVE CESAREAN SECTION AND ITS PROTECTIVE EFFECT}

Colby Perkins, BA, Karyn S. Eilber, MD, Kimberly D. Gregory, MD, MPH, Jennifer T. Anger, MD, MPH

Cedars-Sinai Medical Center, Los Angeles CA

(Presented by: Colby Perkins, BA)

Introduction and Objectives: Studies that evaluated pelvic floor outcomes associated with cesarean section (CS) have varying results. These conflicting results may stem from confounding factors, namely grouping of elective and emergency CS data together and including multiparous and primiparous women in the same data set. We performed a comprehensive literature review to understand the pelvic floor outcomes for primiparous women who received a primary elective CS, emergency CS or who gave birth vaginally.

Methods: Using PubMed and the Cochrane Review, we searched for articles that analyzed data on delivery methods among primiparous women. The following outcomes were evaluated: urinary incontinence (UI), fecal incontinence (FI) and pelvic organ prolapse (POP). Figure 1.

Results: Of the 14 studies included in our review, eleven evaluated UI outcomes, six included outcomes data on FI and no articles were located on POP. Nine studies had short-term follow-up of $<1.5$ years and four studies had follow-up $>1.5$ years and one study had an unknown timeline. For UI, rates after elective CS ranged from 0-33\%, emergency CS 6.5-27.1\% and VB $10.3-54 \%$. Ten of eleven studies found that CS (both elective and emergency) is protective against developing UI compared to VB, two of which had long-term follow-up. Five studies found the risk of developing UI in a primiparous woman after an elective CS versus emergency CS to be statistically similar. Three UI studies found elective CS to provide a statistically significant protective benefit against developing UI compared to emergency CS, two of which say that the CS must be elective in order for the protective benefits against UI to be achieved. For FI, rates after elective CS ranged from $0-51 \%$, emergency CS $0-51 \%$ and VB $4-47.8 \%$. However, five of the six studies on FI show a protective effect of CS, two of which say the rate of FI following elective and emergency CS is statistically similar.

Conclusions: Among primiparous women, CS may be protective against UI and FI. However, there is significant variability in the prevalence of UI and FI between the studies. There is a dearth of data regarding the effect of elective CS on POP among primiparas.

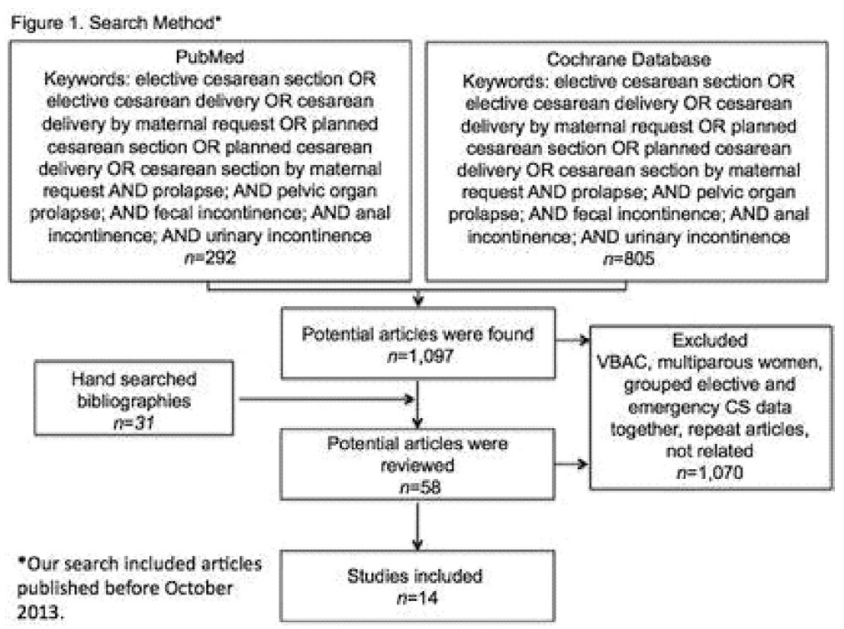

Poster \# NM21

SUCCESS OF AUTOLOGOUS PUBOVAGINAL SLING FOLLOWING FAILED MIDURETHRAL SLING

Jaclyn Milose, MD, John Stoffel, MD, J. Quentin Clemens, MD, MSci and Anne Pelletier-Cameron, MD

University of Michigan

(Presented by: Jaclyn Milose, MD)

Introduction: There is no consensus on the management of stress incontinence status post failed synthetic midurethral sling. It has generally been our practice to perform autologous pubovaginal slings (APVS), however, little empirical work has been performed to assess the efficacy.

Methods: We identified 130 patients who underwent APVS following one or more failed synthetic midurethral sling from 2000 to 2012, 121 of which had follow up. We performed bivariate analysis for factors associated with failure of APVS. Results: Of our patient population 52 (40\%) women had a prior retropubic synthetic sling, 46 (35.4\%) had a transobturator, 13 (10\%) had a ProteGen ${ }^{\circledR}$ sling, four (3.1\%) had a mini sling and seven (5.4\%) had a bone anchored sling. 99 patients (76.2\%) had one prior synthetic sling while 30 patients $(23.1 \%)$ had two or more prior procedures including one with nine prior procedures. Synthetic sling failure was secondary to recurrent or persistent stress incontinence alone in 81 women $(62.3 \%)$, mixed incontinence in three (2\%), recurrent stress incontinence following sling incision for retention in 10 (7.7\%) and infection/ erosion/fistula in 36 (27.7\%). 49 (37.7\%) patients required excision of their synthetic sling removed prior to the autologous sling and $29(22.3 \%)$ required it at the same time as the APVS for either exposure, erosion, pain or retention. On preoperative FUDS (fluoroscopic urodynamics), 61 patients (46.9\%) had urethral hypermobility, 35 (26.9\%) had cystocele, $16(12.3 \%)$ had detrusor overactivity and 46 (35.4\%) had an open bladder neck at rest. The overall patient reported success or improvement rate was $93.4 \%$ (52 had complete resolution of incontinence, 13 reduced leakage, 38 no SUI but persistent UUI, 10 retention). The rate of urgency/urge incontinence following PVS was $31.4 \%$ and the rate of retention was $8.3 \%$. The patients who required mesh removal did not appear to have worse outcomes than those who did not (rate of cure/improvement $85.9 \%$ vs. $88 \% p=0.71$ ). None of the factors examined were associated with sling failure.

Conclusion: APVS is very effective in the management of stress incontinence following failure of a synthetic midurethral sling. 
Even in complex patient populations who have required prior mesh excision, it is a successful treatment for stress incontinence.

Clinical Relevance: Female Urology-including Incontinence

\section{Poster \# NM22}

THE VALUE OF RETROPUBIC SLINGS FOLLOWING FAILED PRIOR ANTI-INCONTINENCE PROCEDURES

Michael Aberger, $\mathrm{MD}^{1}$, William Parker, $\mathrm{MD}^{1}$, Alexander Gomelsky, $\mathbf{M D}^{2}$ and Priya Padmanabhan, MD, MPH ${ }^{1}$

${ }^{1}$ University of Kansas; ${ }^{2}$ Louisiana State University

(Presented by: Michael Aberger, MD)

Objectives: Mid-urethral slings (MUS) are considered first-line surgical treatment of stress urinary incontinence (SUI). However, there is a paucity of data regarding the use of retropubic slings for patients who failed a prior sling. This study compares the outcomes of retropubic mid-urethral slings (SPARC) to autologous pubovaginal rectus fascial slings (pvs) for sling refractory incontinence.

Methods: A retrospective review of 213 consecutive patients undergoing placement of SPARC $(n=152)$ or pvs $(n=61)$ by two fellowship trained urologists for prior failed sling surgery was conducted over an eight-year time span. Mean follow-up was 29 months (range 3 to 93). Pre- and post-operative pad use was recorded for all patients in addition to completion of four validated questionnaires pre- and post-operatively: Stressrelated leak, Emptying ability, Anatomy, Protection, Inhibition, Quality of life, Mobility and Mental status Incontinence Classification System (SEAPI-OMM), Incontinence Impact Questionnaire (IIO-7), Urogenital Distress Inventory (UDI-6) and Visual Analog Score (VAS).

Results: The overall subjective cure rate was $61.1 \%$. 27 total patients $(12.7 \%)$ required additional anti-incontinence procedures and the incidence of de novo urgency was 9.9\% (21 patients). Short-term (<30days) urinary retention was the most common complication recorded (4.04\% of all patients). The number of pads used per day reduced from a mean of 3.26 to a mean of $0.77(p<0.0001)$ post-operatively. A statistically significant improvement between pre- and post-operative SEAPI total, IIQ-7, UDI-6 and VAS was demonstrated ( $p$ $<0.0001)$. Sub-analysis comparing the SPARC to the rectus pvs used for secondary repair demonstrated no significant differences in subjective cure rates, post-operative pad use, SEAPI total, IIO-7, UDI-6 or VAS between groups (Table 1).

Conclusions: The widespread use of surgical slings for SUI has led to an increase in the absolute number of patients who fail their primary procedures. Secondary repair with a retropubic MUS is as durable and effective in improving OoL as the rectus pvs. This provides invaluable information for patient education and surgical planning for refractory stress incontinence.

Clinical Relevance: Female Urology-including Incontinence

\begin{tabular}{|c|c|c|c|}
\hline $175 x$ & AHAt & An & Philut \\
\hline 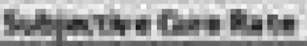 & 6st & 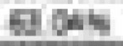 & $9 \mathrm{~s}$ \\
\hline 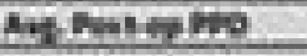 & 14 & 417 & 81 \\
\hline 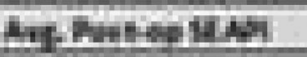 & 14 & 111 & PH \\
\hline Athats Bat & 218 & 213 & 로노 \\
\hline 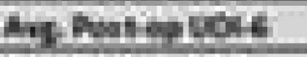 & Eth & 111 & 1日a \\
\hline Aratian & 14. & 811 & 87 \\
\hline
\end{tabular}

\section{Poster \# NM23}

\section{PROSPECTIVE EVALUATION OF PATIENTS' KNOWLEDGE AND PERCEPTIONS OF MESH}

Christopher Tenggardjaja, MD and Howard Goldman, MD

Cleveland Clinic, Cleveland, $\mathrm{OH}$

(Presented by: Christopher Tenggardjaja, MD)

Introduction: In 2011, the U.S. Food and Drug Administration (FDA) released an updated safety communication regarding the use of transvaginal mesh for pelvic organ prolapse (POP) and stress urinary incontinence (SUI). Anecdotally, patients presenting for opinions regarding surgical treatment of incontinence/prolapse often have misconceptions regarding the use of synthetic mesh. Our objective is to prospectively evaluate patients' understanding of mesh using a questionnaire based survey.

Methods: A 25 question based survey was prospectively administered to new female patients presenting to a single academic institution specialty clinic. Patients were invited to participate if they were being seen for either SUI or POP. Established patients were excluded from participation. Demographics and responses were recorded and analyzed. Statistics were utilized Chi-square and Fisher's exact test when appropriate for categorical variables.

Results: Surveys were administered to patients from March 2013 to October 2013. A total of 188 surveys were collected. The typical presenting patient was 60 years old, Caucasian (87\%), married (62\%), with at least a high school education (77\%) and not currently employed (55\%). The majority of patients $(60.1 \%)$ surveyed were being evaluated for urinary incontinence which had not been previously treated. Patients were not necessarily aware of different types of incontinence. 97 patients (52\%) obtained most of their information about mesh from television. The majority of patients said they were unsure if mesh used for SUI was different than that used for POP (62\%), were aware of the FDA safety communication (61\%) and believed there was a recall of mesh (51\%). In patients who were aware of the FDA announcement, 57/97 (75\%) believed there was a recall $(p=0.04)$. For patients who were unsure about different types of mesh use in SUI/POP surgery, they were also unsure or likely to believe there was a recall $(p<0.001)$. Patients exhibited a bimodal distribution when asked about their anxiety of mesh being used in surgery and their concern about mesh use.

Conclusion: The majority of new patients presenting to a specialty female pelvic medicine clinic derive most of their information about mesh from television. Interestingly, those aware of the FDA statement understood that to mean there had been a recall of transvaginal mesh. Furthermore, while many patients had significant concerns about mesh use during surgery, many did not.

Clinical Relevance: Pelvic Organ Prolapse

\section{Poster \# NM24}

WHO ARE "THEY"?: THE EFFECT OF MEDIA ON FLUID INTAKE AMONG WOMEN WITH OAB

Lauren N. Wood, $M^{1}$, Jennifer T. Anger, $M D, M P H^{1}$, Ronit Lyon, $\mathrm{BA}^{2}$ and Karyn S. Eilber, $\mathrm{MD}^{1}$

${ }^{1}$ Cedars-Sinai Medical Center, Los Angeles, CA; ${ }^{2}$ David Geffen School of Medicine at UCLA, Los Angeles, CA

(Presented by: Lauren N. Wood, MD)

Introduction and Objectives: It is a commonly held belief that high volume water intake is beneficial for one's health. 
However, increased fluid intake exacerbates overactive bladder (OAB) symptoms. We sought to determine perceptions regarding fluid intake among women with symptoms of $O A B$ and identify the source of their information.

Methods: Women with symptoms of OAB were identified by chief complaint of frequency, urgency and/or urge incontinence in a tertiary female pelvic medicine practice. Patients were contacted via telephone and administered a survey regarding perceptions of optimal daily water intake, possible benefits of drinking water and where this information was obtained (Figure 1).

Results: Of the 35 women who completed the survey, the majority ( 25 women $=71 \%$ ) believed it was necessary to drink six to eight or more glasses of water daily. Twenty-two (62\%) responders cited the media (news/magazines/television/ books) as the main source of information. Nine (25\%), five $(14 \%)$ and six (17\%) women cited a physician, nutritionist or dietician, and friends or family as providing this information. Two individuals (5\%) referenced a personal trainer. When asked directly whether a physician instructed them to drink a specific number of glasses of water daily, 11 women (31\%) responded "yes." When asked what the health benefits of drinking large quantities of water are, 20 women (57\%) responded "flushing," "cleansing" or "detoxifying" the body.

Conclusions: Women with $\mathrm{OAB}$ falsely believe that drinking large amounts of water is needed for good health. The majority of women obtain their information about water intake outside the healthcare system. Patient re-education regarding fluid intake is an important element of treatment for women with $\mathrm{OAB}$.

Clinical Relevance: Female Urology-including Incontinence

\begin{tabular}{|c|c|}
\hline 1) & What is the optinal anount of water you need to drink to be neathy? \\
\hline 2) & How much water should you dink every day? \\
\hline 3) & Where did you get this intom ation? \\
\hline 4) & Did a physician tell you to dink a specific number of glasses of water? \\
\hline 5) & $\begin{array}{l}\text { Did someone other than a physician telil you to drink a specific number of gasses of } \\
\text { water? }\end{array}$ \\
\hline & How many glasses of water do you drink a day? \\
\hline 7) & How many glasses of cther fluids such as coffee, tea, juice or soda do you dnrk a day? \\
\hline B) & Do you know any health benefits of drinking targe quantities of water? \\
\hline
\end{tabular}

\section{Poster \# NM25}

ADDITION OF A DEFECOGRAPHY PHASE TO DYNAMIC PELVIC MRI ENHANCES DETECTION AND VISUALIZATION OF PELVIC ORGAN PROLAPSE

Hina Arif Tiwari, MD, Bobby Kalb, MD, Joel Funk, MD, Diego Martin, MD, PhD and Christian Twiss, $M D$ University of Arizona College of Medicine, Tucson, AZ (Presented by: Christian Twiss, MD)

Introduction: Dynamic Pelvic MRI (DPMRI) remains an emerging diagnostic modality in the evaluation of Pelvic Organ Prolapse (POP), but study quality remains variable, and is typically dependent upon performance of a proper valsalva maneuver during the dynamic phase of the procedure. We sought to determine whether the addition of a defecography phase (DP) to routine DPMRI would increase its clinical utility over standard valsalva maneuver (VM) in the evaluation of POP.

Methods: Fifty-six female patients undergoing preoperative evaluation for surgical correction of symptomatic POP were evaluated by DPMRI utilizing a protocol incorporating both VM and DP. Ultrasound gel was injected into the vagina and rectum followed by: (1) steady state free precession images in the sagittal plane; (2) dynamic cine acquisitions during VM; and (3) dynamic cine acquisitions during DP. All images were separately reviewed in a blinded fashion by two radiologists experienced in DPMRI. The HMO system was used for quantification of pelvic floor descent. Inferior descent of the ano-rectal junction, bladder base and vaginal vault was recorded in all patients, utilizing the pubococcygeal line (PCL) as a fixed landmark. IRB approval was obtained for the study. Results: DP produced a greater degree of pelvic organ descent compared to VM for all recorded measures in all 56 patients. $100 \%(56 / 56)$ demonstrated rectocele, $71 \%(40 / 56)$ cystocele and $71 \%(40 / 56)$ vaginal vault descent on DP, compared with $86 \%$ (48/56), $14 \%(8 / 56)$ and $43 \%(24 / 56)$ on VM, respectively. The average descent of the bladder base was $3.4 \mathrm{~cm}$ with DP, compared to $0.4 \mathrm{~cm}$ with VM $(p<0.01)$. Average descent of the vaginal vault was $5.1 \mathrm{~cm}$ with DP, compared to $3.5 \mathrm{~cm}$ with VM $(p<0.01)$. Average rectal descent was $5.5 \mathrm{~cm}$ with DP, compared to $4.6 \mathrm{~cm}$ with VM $(p<0.01)$.

Conclusion: DPMRI utilizing a defecography phase demonstrates pelvic organ prolapse better than DPMRI utilizing valsalva maneuver alone. Pelvic organs may show mild prolapse or even appear normal with valsalva maneuver alone, but may demonstrate marked prolapse during the defecography phase.

Clinical Relevance: Pelvic Organ Prolapse

\section{Poster \# NM26}

URINARY NEUROTROPHIC PEPTIDES IN POSTMENOPAUSAL AGE-MATCHED WOMEN WITH AND WITHOUT OVERACTIVE BLADDER

Gina Northington, $\mathrm{MD}, \mathrm{PhD}^{1}$, Jon Pennycuff, BS, $\mathrm{MPH}^{1}$, Stacey Schutte, $\mathrm{PhD}^{1}$, Deborah Karp, $\mathrm{MD}^{1}$, Catherine Hudson, $\mathrm{MD}^{1}$ and Anna Malykhina, $\mathrm{PhD}^{2}$

${ }^{1}$ Emory University, Atlanta, GA; ${ }^{2}$ University of Pennsylvania, Philadelphia, PA

(Presented by: Catherine Hudson, MD)

Objectives: Few studies examining urinary neuropeptides as biomarkers for overactive bladder (OAB) have included agematched controls. The purpose of this study was to compare the expression of urinary brain derived nerve factor (BDNF) and Substance $\mathrm{P}(\mathrm{SP})$ in age-matched postmenopausal women with and without $O A B$.

Methods: After IRB approval, postmenopausal women over the age of 50 with and without $O A B$ were enrolled. OAB symptoms were assessed using the $O A B-q$, a validated questionnaire that reliably discriminates between women with and without $O A B$. Women were excluded if they had a UTI, neurologic disorder, malignancy, renal disorder, diabetes, received treatment for $O A B$ or were taking estrogen. All women were required to complete three questionnaires upon enrollment: The OAB-O, IIO-7 and PFDI-20. Demographic and clinical data were recorded. Enzyme-linked immunosorbent assay (ELISA) was used to determine the expression of urinary neuropeptides which were normalized to creatnine. Ouestionnaire scores and urinary neuropeptides were compared between women with and without OAB. Multivariate models were developed to determine independent risk factors for increased BDNF or SP. Results: Fifty five similarly aged women were included in this pilot study: 23 with $\mathrm{OAB}$ (age $63.3+/-8.6 \mathrm{yrs}$ ) and 32 controls (age $63.7+/-9.2), P=0.85$. Women with $\mathrm{OAB}$ had a mean $(+/-$ SD) score $(\max 100)$ on the OAB-q of $57.6+/-21.2$ vs. $9.1+/-$ 8.9 for the controls $(p<0.0001)$. Scores on the IIQ-7 and UDI-6 were also significantly lower in the controls (all $p<0.0001$ ). The $\mathrm{OAB}$ group consisted of significantly more African Americans 
(24\% vs.13\%, $\mathrm{P}=0.01)$, less women with pelvic pain $(29 \%$ vs. $53 \%, P=0.05)$, and less women with prior pelvic surgery (11\% vs. 36\%, p <0.01). There were no significant differences in BMI, smoking and parity. Mean +/-SEM urinary expression of BDNF and SP were not significantly different between the $\mathrm{OAB}$ and control groups $(4.13+1.0$ vs. $3.8+0.8 \mathrm{pg} / \mathrm{ml}$, $\mathrm{P}=0.8$ and $0.3+0.06$ vs. $0.5+0.11, \mathrm{P}=0.2$, respectively). On multivariate analysis increasing age was independently associated with urinary BDNF $(\mathrm{P}=0.02)$ while pelvic pain $(P=0.01)$ and smoking $(P=0.02)$ were associated with urinary SP.

Conclusions: In similarly aged postmenopausal women, BDNF and SP may not serve as discriminatory biomarkers for OAB. Demographic and clinical factors should be considered in the evaluation of novel urinary biomarkers for OAB.

Clinical Relevance: Female Urology-including Incontinence

\section{Poster \# NM27}

\section{BIRTH METHOD CHOICES, COMPLICATIONS, AND LONG- TERM SEOUELAE: ARE WOMEN FULLY INFORMED?}

Colby Perkins, BA, Jennifer T. Anger, MD, MPH, Kimberly D. Gregory, MD, MPH and Karyn S. Eilber, MD Cedars-Sinai Medical Center, Los Angeles CA

(Presented by: Colby Perkins, BA)

Introduction and Objectives: Approximately 1\% of US women had an elective cesarean section (CS) in 2011-2012, despite $21 \%$ of U.S. obstetricians (OBs) recommending an elective CS for themselves or their partners, citing concern for urinary and fecal incontinence and pelvic organ prolapse (Wax, 2005). As $83 \%$ of women obtain pregnancy-related health information from the internet (Lagan, 2010), we evaluated the ability of internet resources to inform women of their birth method choices, which in turn may shed some light on the disparity between patient elective CS rates and OB personal preference for elective CS.

Methods: For this descriptive study, we searched Google for patient health education sites and evaluated the top 20 hits for each search. We also evaluated the pregnancy-related content of the top nine websites for general health (Consumer and Patient Health Information Section (CAPHIS), 2013). See Figure 1.

Results: Of the 49 sites identified, we evaluated 27 health websites. Ninety-three percent (25/27) of the sites mentioned risks associated with CS. Seventy-eight percent (21/27) of the sites included information about immediate complications (tears, episiotomies) of vaginal birth (VB) on their main pregnancy page. Of the sites that included complications of VB, $43 \%(12 / 21)$ of them acknowledged the potential for pelvic floor issues such as urinary incontinence and pelvic organ prolapse on their main pregnancy page. Only $22 \%$ (6/27) of the sites mention fecal incontinence as long-term complication of VB on their main pregnancy page. Twenty-six percent (7/27) of sites present elective CS as a potential birth option on their main pregnancy page.

Conclusions: Using internet resources alone, data does not appear to present a balance of information regarding risks of different delivery methods. Risks of VB appear to be minimized, and few sites acknowledge elective CS as a birth option. More research is needed regarding what women want and need to know for effective shared decision making pertinent to childbirth.

Clinical Relevance: Pelvic Organ Prolapse

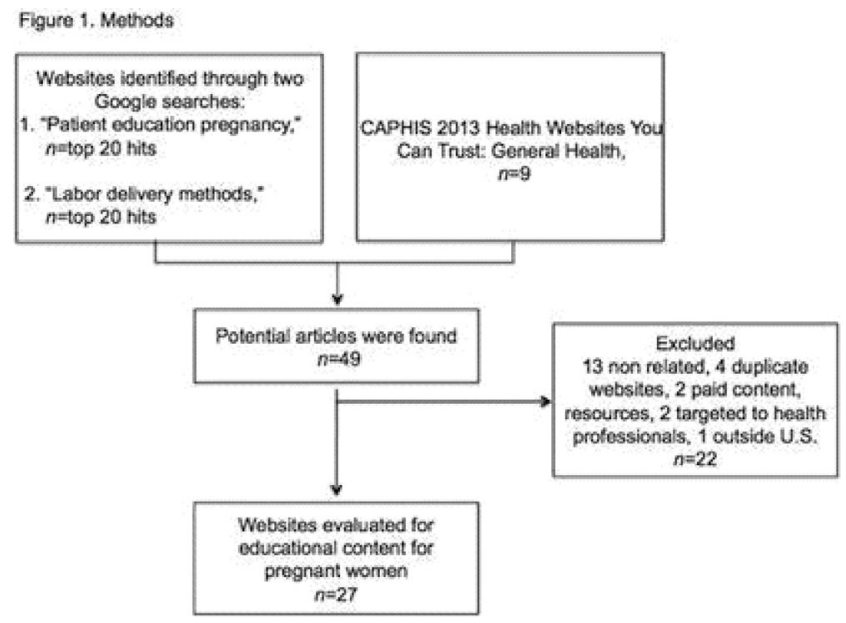

Poster \# NM28

IMPROVEMENT EVALUATION AFTER MIDURETHRAL SLING: WHAT IS THE CORRELATION BETWEEN UROLOGIST PERCEPTION AND UDI-6?

Paholo Barboglio Romo, $\mathrm{MD}, \mathrm{MPH}^{1}$ and Veronica Triaca, $\mathrm{MD}^{2}$

${ }^{1}$ Dartmouth-Hitchcock; ${ }^{2}$ Concord Hospital

(Presented by: Paholo Barboglio Romo, MD, MPH)

Introduction and Objectives: The UDI-6 is a composite of irritative, stress incontinence (SUI) and obstructive symptom subscales. We hypothesize that physicians underestimate patient's urinary symptoms and quality of life (OoL).

Methods: IRB approved, single surgeon's retrospective review of a cohort of 275 women undergoing mid-urethral sling. Selfreported pre and postoperative questionnaires (UDI-6) were available for review. All women had demonstrable SUI in the office and MUI diagnosis was established clinically. We assessed urologist perceived improvement with chart reviews. We looked at UDI-6 items 3 and 4 to address the SUI symptoms. Urologist assessment at one year were dichotomized to "not improved" (0) or "improved" (1) on the areas of SUI and MUI. UDI-6 answers were categorized as No/Not at all/Slightly as "improved" (1) and answers Moderately/Greatly as "not improved" (0). Clinical significance was based on the Urinary Incontinence Network parameter of at least $70 \%$ decrease in UDI-6 total score. Pearson's correlation coefficient was used to correlate urologist assessment of symptoms with UDI-6 items \#3 (SUI) and \#4 (small leak). A coefficient of 1 suggests a very strong correlation.

Results: Data was available for 209/275 patients who underwent DUPS for MUI $(n=157)$ or SUI $(n=52)$. Mean age was 57 years (23-87). Urologist's perceived improvement at one year was $>90 \%$ ( $p=0.87)$. Improvement $(>70 \%)$ on UDI-6 total score was $71 \%$ in MUI and $79 \%$ in genuine SUI $(p=0.373)$. However, in specific subscale items \#3 and \#4, improvement (>70\%) became significantly higher $(p<0.001)$ in the MUI. There was a moderate correlation between the urologist's assessments and women with improvement ( $>70 \%)$ in the UDI-6 total score in both MUI $(r=0.39)$ and genuine SUI $(r=0.38)$ groups. The correlation was strong for items \#3 $(r=0.61)$ and \#4 $(r=0.55)$ in the MUI group and was strong for $\# 4(r=0.55)$ in those with pure SUI. The correlation was even stronger $(r=0.88)$ with item\#3.

Conclusions: Urologist reported post-operative improvement at one year correlates moderately with patient's total UDI-6 scores in women who underwent DUPS. Although the use of 
UDI-6 specific subscales has not been validated, our data suggests that these maybe a more specific tool to evaluate patient's post-operative outcomes.

Clinical Relevance: Female Urology-including Incontinence

\section{Poster \# NM29}

\section{MESH PERFORATION AFTER PELVIC SURGERY: TROCAR INJURY MATTERS}

Catherine Harris, $\mathrm{MD}^{1}$, David Osborn, $\mathrm{MD}^{2}$,

Stephen Mock, $\mathrm{MD}^{2}$, Melissa Kaufman, $\mathrm{MD}^{2}$, Nicholas Smith ${ }^{2}$, Roger Dmochowski, $\mathbf{M D}^{2}$ and W. Stuart Reynolds, $\mathbf{M D}^{2}$

${ }^{1}$ Nashville, TN; ${ }^{2}$ Nashville, TN

(Presented by: Catherine Harris, MD)

Objective: To evaluate technical and patient factors that impact the development of mesh perforation compared to exposure in patients after synthetic mesh mid-urethral sling or pelvic organ prolapse kit placement utilizing a trocar.

Methods: A retrospective review of consecutive patients undergoing surgery for mesh perforation or mesh exposure at a single institution between 2003-2012. Mesh perforation was defined as mesh within the lumen of the bladder or urethra, with exposure defined as mesh visible through separated vaginal epithelium. Variables between patients with mesh perforation and mesh exposure were compared with chisquared tests. The risk of mesh perforation over exposure was analyzed with multivariate logistic regression, adjusting for age, body mass index (BMI), smoking status, diabetes, type of sling placed, type of surgeon and trocar injury at the time of mesh placement.

Results: Eighty-three women were identified, 28 with mesh perforation and 55 with mesh erosion. The average age was 49.8 years. Median time from mesh placement to revision was 32.5 months (IOR 38.5). Patients' average BMI was 29.2, 30\% were smokers and $13 \%$ were diabetic. Ten (42\%) patients in the perforation group and two (4\%) patients in the exposure group had a trocar removed and replaced for being too close to the bladder or urethra at the time of mesh placement (Chi-Squared $p<0.001$ ). Voiding complaints were the presenting symptom in 15 of the 28 patients with mesh perforation compared to one patient with mesh exposure (Chi-Squared $p<0.001)$. After multivariate logistic regression analysis, trocar injury (OR 15.7, 95\% CI 2.25-109.25, p 0.005) and diabetes (OR 10.5, 95\% CI 1.2290.3 , p 0.032) were associated with an increased risk of mesh perforation. BMI (OR 0.87, 95\% CI 0.77-0.98, p=0.024) was associated with a decreased risk of mesh perforation. Smoking status, the type of performing surgeon and transobturator versus retropubic trocar placement were not found to be significant risk factors for mesh perforation.

Conclusions: Our analysis suggests the risk for mesh perforation after a pelvic support procedure is increased in patients with diabetes and trocar injury at the time of the procedure and inversely related to BMI. Trocar injury at the time of pelvic surgery has not been considered to increase the risk of an adverse outcome; however, our study suggests that this may not be the case.

Source of Funding: None

Clinical Relevance: Female Urology-including Incontinence

\section{Poster \# NM30}

ENDO-LUMINAL ULTRASOUND FOR EVALUATION OF FEMALE URETHRAL DIVERTICULA COMPARED WITH MRI
Michael J. Amirian, MD, Alana M. Murphy, MD, Patrick J. Shenot, MD and Akhil K. Das, MD (Presented by: Michael J. Amirian, MD)

Introduction and Objectives: Although the true prevalence is unknown, female urethral diverticula (UD) is reported to occur in up to 1 to $6 \%$ of adult females. It carries a significant amount of morbidity and distress to the patient if misdiagnosed, and as a result managed inappropriately. Numerous modalities have been used in an attempt to image UD, but no imaging technique is considered the gold standard. Magnetic resonance imaging (MRI) is often used and offers high resolution images in a noninvasive approach. However, obtaining an MRI is not feasible in many instances relating to the very high cost and certain contraindications. This may result in a delay in diagnosis and increasing morbidity for the patient. Ultrasound has been shown to be a sensitive and inexpensive imaging modality in various platforms. We aim to evaluate the accuracy, benefits and versatility of endo-luminal ultrasound of the urethra with a catheter-based transducer in the diagnosis and management of UD compared to MRI.

Methods: At the time of surgical repair of UD, the urethra was catheterized directly with a $6.2 \mathrm{Fr}(12.5 \mathrm{MHz})$ catheter-based ultrasound transducer to generate 360 degree transaxial realtime images (see Image 1). These images were used in identification of the UD intraoperatively by the surgeon. These patients had confirmed UD on preoperative MRI during initial work up.

Results: The accuracy of catheter-based endo-luminal ultrasound in identifying UD was compared to preoperative MRI in three female patients. In all three instances, endo-luminal ultrasound was diagnostic in recognizing UD that had been confirmed on MRI. In addition, intraoperative monitoring by the surgeon allowed for refining of the surgical approach during the case.

Conclusions: Endo-luminal ultrasound is a valuable and accurate diagnostic tool for UD compared to MRI. In addition, it can offer realtime imaging assistance in the operating room to facilitate surgical repair. Endo-luminal ultrasound may also offer a more rapid and lower cost alternative to MRI in the office setting for prompt identification of UD.

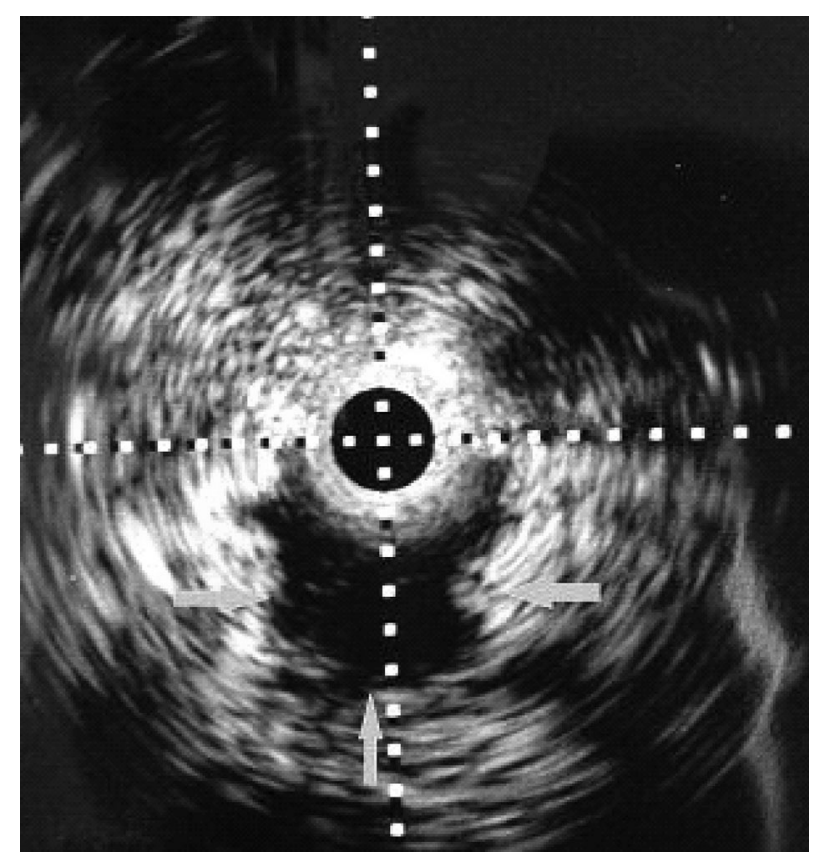




\section{Poster \# NM32}

\section{PATIENT EXPOSURE TO PELVIC ORGAN PROLAPSE SURGERY ON YOUTUBE}

Natasha Ginzburg, MD, Peter O'Hare, MD, Darlene Morrissey, DO and Kristene Whitmore, MD

Drexel University College of Medicine, Philadelphia, PA (Presented by: Natasha Ginzburg, MD)

Introduction and Objectives: Patient access to online related health information has exploded via contact with websites, blogs, social media and video outlets. Many technically focused surgical videos are also available for both physician and patient viewing. Pelvic organ prolapse is unique in its sensitive nature, graphic videos and current legal controversies. We analyzed the currently available videos on YouTube to determine their character, popularity and relevance.

Methods: A cross-sectional analysis of YouTube was conducted by using the search terms "pelvic organ prolapse surgery," "cystocele surgery" and "rectocele surgery". The matching videos in each category were examined and sorted by relevance, length and popularity. Videos with fewer than five views were excluded. The themes of the videos were classified as educational (main focus on patient education), technical/ surgical (focus on technique, intended for practitioners), marketing/advertising, legal or other.

Results: A YouTube search was performed using the terms above returning 3,950 videos. After filtering by relevance and excluding all videos with less than five views, removing duplicates; a total of 579 videos were examined, sorted by length and popularity. 230 videos that were classified as "other" were excluded, leaving 349 videos. Patient educational videos accounted for 142 videos (40.7\%), while there were 121 surgical technique videos (34.7\%). Legal videos accounted for 54 (15.5\%), while direct marketing accounted for the remaining 32 (9.2\%). Total video views were $5,247,501$. Of the total views, $1,243,727$ (23.7\%) were educational videos, 3,481,140 (66\%) surgical/technical videos, 92,837 (1.8\%) legal videos and 429,797 (8.2\%) direct marketing videos.

Conclusions: Given the extensive number of YouTube video views, patients are clearly gaining access not only to material intended for the lay person, but also to surgical and technical videos demonstrating live procedures, including laparoscopic and vaginal. These patients are additionally exposed to legal advertisements with graphic visual descriptions of risks of these procedures. Despite the large number of these legal videos, they are still the minority of views, suggesting that many visitors are not interested. Now, more than ever, it is imperative that the practitioner is aware of these information sources in order to communicate effectively and provide the patient with informed surgical consent utilizing AUGS guidelines.

Clinical Relevance: Pelvic Organ Prolapse

\section{Poster \# NM33}

\section{RISK FACTORS FOR PERIOPERATIVE COMPLICATIONS AFTER UROGYNECOLOGIC SURGERY}

Tatiana Catanzarite, MD, Umang Jain, BS ${ }^{1}$, Margaret Mueller, $\mathbf{M D}^{2}$, Aksharananda Rambachan, BA $^{1}$, John Kim, $\mathbf{M D}^{1}$ and Kimberly Kenton, $\mathrm{MD}, \mathbf{M S}^{2}$

${ }^{1}$ Northwestern University Feinberg School of Medicine, Department of Surgery, Chicago, IL; ${ }^{2}$ Northwestern University Feinberg School of Medicine, Department of OB/GYN, Chicago, IL (Presented by: Tatiana Catanzarite, MD)
Objective: To determine rates of and risk factors for complications after prolapse and incontinence procedures utilizing the ACS-NSOIP database.

Methods: We performed a retrospective review of the NSOIP database between 2006 and 2010 for all patients undergoing urogynecologic procedures. Patients were identified using the Current Procedural Terminology (CPT) codes for suburethral sling, vaginal vault suspension, anterior/posterior/enterocele/ paravaginal repair, anal sphincteroplasty, fistula repair, insertion/revision of vaginal graft, Interstim implantation, urethral procedures, sacral colpopexy, colpectomy, colpocleisis and vaginal, laparoscopic and abdominal hysterectomy with any concomitant urogynecologic procedure. Primary outcomes of interest were 30-day complication rates. Patient demographics, comorbidities, and procedural variables were tracked. Bivariate and multivariate analyses were performed to identify risk factors for complications.

Results: 19,174 patients underwent urogynecologic procedures within the NSOIP database between 2006 and 2012. Procedures by type are listed in Table 1 . The overall complication rate was $6.09 \%$. The most common complications were urinary tract infection (3.87\%), surgical site infection (1.19\%) and blood transfusion (0.69\%). There were eight deaths, for a mortality rate of $0.04 \%$. Patient-related and procedural characteristics associated with an increased rate of complications on bivariate analysis were evaluated on multivariate regression. After multivariate regression, the variables that emerged as independent risk factors for complications included steroid use (OR 1.79, CI 1.22-2.62, $p=0.003$ ), wound infection (OR 2.1, CI 1.14-3.87, $p=0.017$ ), ASA level above 3 (OR 1.43, CI 1.231.66, $\mathrm{p}<0.001$ ), longer operative time (OR 1.003, CI 1.002$1.003, p<0.001$ ), concomitant procedures (OR 1.16, CI 1.009$1.32, \mathrm{p}=0.037$ ) and resident involvement (OR 1.195, CI 1.041.38, $\mathrm{p}=0.013$ ).

Conclusions: Complication rates after female pelvic organ prolapse and incontinence procedures are low and mortality is rare. We have identified factors that independently correlate with complications, providing important data for surgeons as they counsel patients and consider treatment alternatives.

\begin{tabular}{|c|c|c|c|}
\hline Thes of worpery & CPICAdh & 4 & in-1a174: \\
\hline $\begin{array}{l}\text { Whinaraplical } \\
\text { repuln }\end{array}$ & 9262,3720 & $d x$ & 2460 \\
\hline $\begin{array}{l}\text { Mis apieal } \\
\text { ropant }\end{array}$ & STats & $3 \$ 1$ & 3090 \\
\hline Ablominal & sines: & 36: & Toth \\
\hline $\begin{array}{l}\text { Sala-spetife } \\
\text { repaini }\end{array}$ & 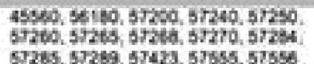 & $46 \mathrm{n}$ & $2100 \%$ \\
\hline $\begin{array}{l}\text { Suspemsios of } \\
\text { uhenes }\end{array}$ & SQ100, 8410 & 48 & 00 st \\
\hline $\begin{array}{l}\text { Younal mas } \\
\text { phetmetht }\end{array}$ & $526 ?$ & 10 & 054 \\
\hline Yoginal moak & 5216, 57\% & 38 & 0954 \\
\hline $\begin{array}{l}\text { Ineonainumst } \\
\text { prosedures }\end{array}$ & 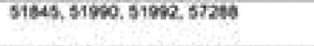 & Tont & $2 \mathrm{si}$ \\
\hline Fitule repain & 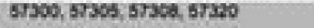 & 12) & acos \\
\hline $\begin{array}{l}\text { Aad ipsineser } \\
\text { proesdurtie }\end{array}$ & 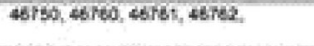 & कot & $360 \mathrm{~h}$ \\
\hline $\begin{array}{l}\text { Unteral } \\
\text { procedures }\end{array}$ & 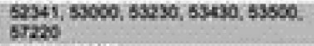 & 110 & $0.97 \%$ \\
\hline $\begin{array}{l}\text { inplantotes of } \\
\text { neureditimulator }\end{array}$ & ents, usin & 11 & bots \\
\hline $\begin{array}{l}\text { Wopladi closwere } \\
\text { procedure }\end{array}$ & 5710,5710 & 351 & Qden \\
\hline \multicolumn{4}{|l|}{ 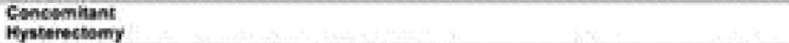 } \\
\hline vegina & 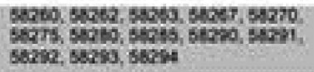 & mh & 14.5T/4 \\
\hline Asdominal & 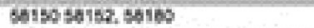 & tor: & $2 \tan$ \\
\hline Lipzesesple & 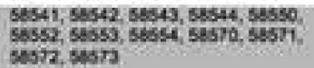 & 1510 & rapes \\
\hline
\end{tabular}




\section{Poster \# NM34}

\section{DETERMINANTS OF EFFICIENCY IN ROBOTIC SURGERY}

Lauren N. Wood, $\mathrm{MD}^{1}$, Juzar Jamnagerwalla, $\mathrm{MD}^{1}$, Ken Catchpole, $\mathrm{PhD}^{1}$, Catherine Bresee, $\mathrm{MS}^{2}$, Bruno Gross, $\mathrm{BA}^{3}$, Stephanie D. Chu, $\mathrm{MD}^{4}$, Karyn S. Eilber, $\mathrm{MD}^{1}$

and Jennifer T. Anger, $\mathrm{MD}, \mathrm{MPH}^{1}$

${ }^{1}$ Cedars-Sinai Medical Center, Los Angeles, CA; ${ }^{2}$ Cedars-Sinai Biostatistics \& Bioinformatics Research Center; ${ }^{3}$ Texas A\&M Health Science Center College of Medicine; ${ }^{4} U C L A$, Los Angeles, CA

(Presented by: Lauren N. Wood, MD)

Introduction and Objectives: Robotic surgery has a variable learning curve with multiple factors potentially affecting operative times. We sought to measure effect of patient, provider and system-related variables on operative time.

Methods: Retrospective data was collected over a 3.5 year period on 1,099 patients undergoing 11 robotic surgeries by 23 urologists and gynecologists at Cedars-Sinai Medical Center in Los Angeles. Data included patient age, BMI, comorbidities, operative time, surgeon volume and type of robot (da Vinci Standard vs. da Vinci S System). Analyses were performed by linear regression modeling.

Results: Average procedure time was $4.87+/-1.33$ hours. Surgeons performed an average of $49+/-83$ surgeries (range 1 to 362 , median $=8$ ). The upper 25 th percentile of surgeons by volume performed 60 or more procedures, and the lower 25 th percentile performed four or less. Patients with $25<\mathrm{BMI}<30$ had procedure times $0.14+/-0.7$ hours longer vs. BMI $<25$ $(p=0.040)$. Surgical time for obese patients $(B M I>30)$ was 0.35 $+/-0.08$ hours longer vs. BMI $<25(p<0.001)$. Diabetes was also associated with increased procedure time, but age and heart disease were not. After performing a subset analysis of surgeons who performed 20 or more surgeries $(n=10)$, the average procedure time for the first 10 surgeries was $4.82+/-$ 2.83 hours and for the last 10 was $5.03+/-2.90$ hours. The upper $(n=5)$ and lower $(n=6) 25$ th percentile of surgeons had an average procedure time of $5.31+/-0.74$ hours and $4.36+/-$ 0.77 hours, respectively $(p=0.067)$. After accounting for covariates such as robot type and BMI, high volume surgeons took $0.90+/-0.44$ hours longer than low volume surgeons $(p=0.042)$. The average time for the da Vinci Standard was 4.70 $+/-1.12$ hours ( $n=639$ procedures) and for the da Vinci $S$ was $5.10+/-1.55$ hours $(n=493$ procedures $)(p<0.001)$. After accounting for surgeon volume, BMI, department and procedure type, the first generation da Vinci Standard had procedure times $0.35+/-0.08$ hours shorter than the second generation da Vinci S ( $p<0.001)$.

Conclusions: Unexpectedly, surgeon operative times did not tend to improve over time, and operative times were not significantly different between high and low volume surgeons. After overcoming learning curves, surgeons may have an inherent "pace" that substantially affects operative time. Clinical Relevance: Female Urology-including Incontinence

\section{Poster \# NM35 \\ RECURRENT STRESS URINARY INCONTINENCE AFTER TRANSVAGINAL MESH REVISION: A COMPARISON OF TREATMENT PARADIGMS}

Elizabeth Brown, MD, MPH, Ryan Krlin, MD and J Christian Winters, MD

Louisiana State University, New Orleans, LA

(Presented by: Elizabeth Brown, MD, MPH)
Introduction and Objectives: Transvaginal mesh placement for the management of stress urinary incontinence (SUI) can result in complications such as pain, obstruction and erosion (3-35\%.) Mesh removal or revision may result in the recurrence of SUI. Many patients require secondary treatment of SUI. In order to decrease the occurrence of recurrent SUI and the need for secondary procedures, we are examining the effect of transvaginal mesh revision with concomitant urethral bulking agent injection. The objective is to determine if this practice reduces the incidence of secondary SUI and the need for retreatment.

Methods: A retrospective chart review was performed to stratify patients undergoing transvaginal mesh sling revision into those patients who underwent transvaginal mesh revision alone and those who underwent transvaginal mesh revision and a prophylactic urethral injection to prevent SUI. SUI outcomes and the need for retreatment were analyzed between groups to determine if there was a treatment effect.

Results: A total of 41 patients met inclusion criteria with an average follow-up of 11 months. 35 patients (85\%) were managed by mesh revision alone. 9/35(25.7\%) reported no incontinence after mesh revision alone. 26/35 (74.3\%) reported incontinence after mesh revision. Of these patients, 13 have not required a secondary procedure. 13/35 (37.1\%) have required a secondary procedure after mesh revision alone. Three (8.5\%) patients underwent sling (two fascial and one midurethral) placement and required no further intervention; 10 (28.5\%) underwent urethral bulking agent injection, with $70 \%$ of these requiring at least one further procedure. Six patients (14.6\%) elected to undergo prophylactic bulking agent injection at the time of mesh revision $-/ 6(66.7 \%)$ of these patients have no recurrent SUI, $2 / 6$ (33.3\%) have minimal SUI, and 100\% have required no further procedures.

Conclusions: The incidence of recurrent incontinence after transvaginal mesh excision is high. Our clinical experience has shown that a sling may be more effective as a secondary procedure for recurrent SUI. Prophylactic urethral bulking agent injection appears to reduce the rate of secondary incontinence.

Clinical Relevance: Female Urology-including Incontinence

\section{Poster \# NM36}

MANAGEMENT OF URINARY FISTULAS DUE TO MESH SLING SURGERY

Gabriel Mekel, $\mathbf{M D}^{1}$ and Jerry Blaivas, $\mathbf{M D}^{2}$

${ }^{1}$ Institute for Bladder and Prostate Research, New York, NY;

${ }^{2}$ Weill Cornell Medical College, New York, NY

(Presented by: Gabriel Mekel, MD)

Introduction and Objectives: Urinary fistulas are rare complications after sling surgery, but there is a paucity of information about their diagnosis and management in the literature. The purpose of this study is to provide a detailed analysis of the management of urinary fistulas that occurred after mesh sling surgery.

Methods: This is a retrospective review of consecutive patients referred because of complications after mesh sling surgery who were found to have urinary fistulas. Electronic medical records and billing records were searched for prior mesh surgery and urinary fistulas. The following data was analyzed: type of sling, mode of presentation, the time interval from the mesh surgery to the appearance of symptoms and diagnosis and clinical and operative findings. Symptomatic outcomes were assessed by the Patient Global Impression of Improvement (PGI-I). 
Surgical outcomes of the fistula repair were based on history and exam.

Results: Nine women were identified (mean age 60; range 3770). Five had more than one fistula. There were a total of seven urethrovaginal fistulas, five vesicovaginal fistulas, one enterovesical fistula and one ureterovaginal; seven patients underwent fistula repair with removal of the entire vaginal portion of the sling (6), Martius flap (6), omental flap (1) and autologous pubovaginal sling (7). Of the remaining two patients, one underwent primary continent urinary diversion and the other is pending surgery. The time interval from mesh placement to onset of symptoms and to fistula repair ranged from 0-10 months (mean 2,9 months) and 4-36 months (mean 15 months), respectively. Follow up range was 1-7 years (mean 3 years). All patients presented with stress incontinence (SUI), four with overactive bladder (OAB), two with voiding symptoms (VS) and one with pain. Five patients had polypropylene slings, two polytetrafluoroethylene, one polyester and one cadaveric. Only two fistulas were visible on exam. Tissue ingrowth into the sling was found in $6 / 8$ patients. All patients in whom the sling was removed had a successful repair (6); in one this was not done and it failed. A successful outcome with respect to symptoms was achieved in 5/7 (SUI), 2/4 (OAB), 2/2 (VS) and 1/1 (pain).

Conclusion: Repair of urinary fistulas complicating sling surgery is daunting. Even in patients with successful repair, almost one third and half of them will have persistent SUI and OAB.

Clinical Relevance: Female Urology-including Incontinence

\section{Poster \# NM37}

\section{THE EFFECT OF VAGINAL ESTROGEN ON URINARY NERVE GROWTH FACTOR AND SYMPTOMS IN WOMEN WITH OVERACTIVE BLADDER}

Jon Pennycuff, BA, $\mathrm{MPH}^{1}$, Stacey Schutte, $\mathrm{PhD}^{1}$, Deborah Karp, $\mathrm{MD}^{1}$, Catherine Hudson, $\mathrm{MD}^{1}$, Anna Malykhina, $\mathrm{PhD}^{2}$ and Gina Northington, $\mathrm{MD}, \mathrm{PhD}^{1}$ ${ }^{1}$ Emory University, Atlanta, GA; ${ }^{2}$ University of Pennsylvania, Philadelphia, PA

(Presented by: Catherine Hudson, MD)

Objectives: Vaginal estrogen therapy has been used as a therapy for overactive bladder (OAB) in postmenopausal women and has been shown to improve lower urinary tract symptoms. Estrogen's effect on neural function is one mechanism by which vaginal estrogen may improve symptoms. The purpose of this pilot study was to determine changes in urinary nerve growth factor (NGF) and OAB symptoms in women after six weeks of treatment with vaginal estrogen.

Methods: Eighteen postmenopausal women over the age of 50 with symptoms of $O A B$ were recruited to participate in this study. OAB symptoms were assessed using the symptom severity subscale of the $O A B-q$, a validated questionnaire that reliably discriminates between women with and without OAB. Women were excluded if they had a UTI, neurologic disorder, malignancy, renal disorder, diabetes, had been treated for OAB (anticholinergics or neuromodulation) or were taking estrogen within three months of enrollment. All women were required to complete two questionnaires upon enrollment - the OAB-q and Pelvic Floor Distress Inventory (PFDI-20) - and treated with vaginal estrogen for six weeks following enrollment. At six weeks, urinary NGF and symptoms were measured. Enzyme-linked immunosorbent assay was used to determine the expression of urinary NGF and creatnine (expressed as mean +/-SEM pg/ml). Pre-and post-treatment questionnaire scores and urinary NGF were compared.

Results: Mean (+/-SD) ages and BMI were $64+/-7$ and $28+/-$ 7 , respectively, at baseline. Mean questionnaire scores for $\mathrm{OAB}-$ $q$ and PFDI 20 at base line were, $30+/-11(\max 100)$ and 116 $+/-34$ (max 300), respectively. Eight women had complete data at follow up. Urinary NGF protein were significantly higher post-treatment with vaginal estrogen when compared to pre-treatment $(15.9+/-5.0$ vs. $3.1+/-1.6 \mathrm{pg} / \mathrm{ml}, \mathrm{P}=0.007$, respectively). Urinary and pelvic floor symptoms measured on the OAB-q and the PFDI-20 were significantly lower after six weeks of estrogen therapy compared to pre-treatment scores (all $\mathrm{p}<0.006$ ).

Conclusions: This pilot study of postmenopausal women suggests that one mechanism by which vaginal estrogen may exert an effect on the urinary tract is by upregulating urinary NGF. Larger prospective studies are required to fully elucidate the mechanisms of vaginal estrogen on bladder function.

\section{Poster \# NM38}

WITHDRAWN

\section{Poster \# NM39}

\section{SURGICAL MANAGEMENT OF URETHRAL PROLAPSE:} EXPERIENCE AT THE UNIVERSITY OF MICHIGAN

Tola Oyesanya, BS and Anne Pelletier Cameron, MD

Department of Urology, University of Michigan Health System, Ann Arbor, MI

(Presented by: Tola Oyesanya, BS)

Introduction and Objectives: Urethral prolapse and caruncles are typically asymptomatic physical exam findings reported mainly in young girls and post-menopausal women. The condition is thought to be idiopathic, but may be attributed to episodic increases in intra-abdominal pressure, such as coughing or constipation. Most patients remain asymptomatic and usually do not seek medical care until symptoms such as bleeding, pain, voiding dysfunction or dysuria develop. Few case studies have explored clinical outcomes after surgical excision. Here we present a case series describing 20+ years of experience in surgical management of urethral prolapse at a tertiary care referral center. The objective is to characterize the demographics, clinical presentation and surgical outcomes of patients who present with urethral prolapse.

Methods: Retrospective review of medical records from female patients who underwent surgery for a symptomatic urethral prolapse or caruncle from June 1995 to March 2013.

Results: Average age of the 23 patients was 35.7 years (3.081.0). Nine of the 23 patients were premenarchal and seven had reached menopause. Three patients were status post excision of urethral prolapse with new recurrence of symptoms. In pediatric patients, three of 23 patients had a positive history of constipation and/or straining; five patients had history of chronic cough. Ten patients underwent conservative management with exogenous estrogen prior to surgery, with an average of three months of conservative treatment. Surgical technique typically involved placement of absorbable sutures while applying traction to the prolapsed mucosa, then amputation of the urethral prolapse in quadrants. Interrupted 4-0 Vicryl sutures were used to re-approximate the mucosa to the surrounding epithelium. Post-operatively, three patients had temporary lower urinary symptoms/bleeding; one patient required a visit to the emergency room. Long term, two patients had recurrence of urethral prolapse and one patient developed a urethral stricture. 
Conclusions: Surgical excision of urethral prolapse in symptomatic individuals is a safe procedure. Patients tend to do well postoperatively, but recurrence of prolapse is a possibility of which patients must be counseled.

Clinical Relevance: Female Urology-including Incontinence
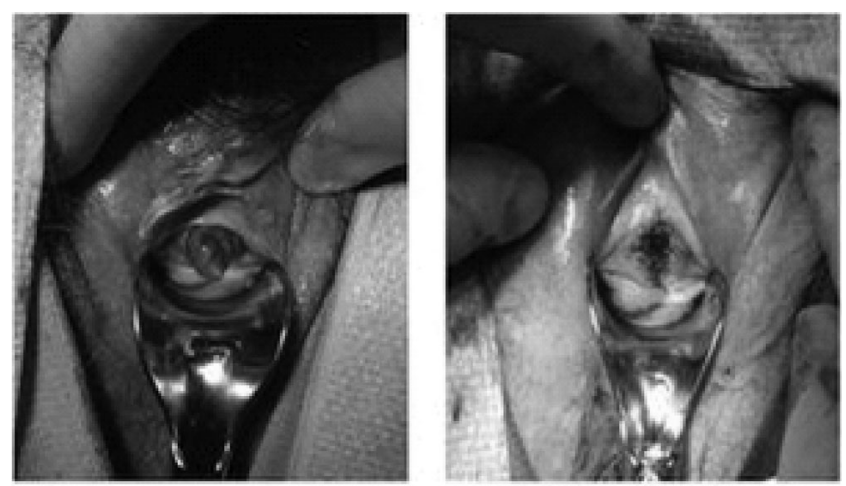

Figure 1. Urethral prolapse in a healthy. postmenopausal woman, shown here preoperatively (left) and immediately postoperatively (right).

\section{Poster \# NM40}

TRANSURETHRAL RADIOFREOUENCY COLLAGEN DENATURATION IN THE TREATMENT OF MILD TO MODERATE FEMALE STRESS URINARY INCONTINENCE: TWELVE-MONTH INTERIM ANALYSIS

James Lukban, $\mathrm{DO}^{1}$, Peter Takacs, $\mathrm{MD}^{2}$, Monisha Crisell, $\mathrm{MD}^{3}$, Douglas Van Drie, $\mathrm{MD}^{4}$, Denise Elser, $\mathrm{MD}^{5}$, Phillip Bressman, $\mathrm{MD}^{6}$, Royce Adkins, $\mathrm{MD}^{6}$ and Randall Kahan, $\mathbf{M D}^{7}$

${ }^{1}$ Division of Urogynecology, Eastern Virginia Medical School; ${ }^{2}$ Division of Urogynecology, Eastern Virginia Medical School, Norfolk, VA; ${ }^{3}$ Tri Valley Urology Medical Group, Murrieta, CA; ${ }^{4}$ Female Pelvic Medicine \& Urogynecology, Grand Rapids, MI; ${ }^{5}$ Women's Health Institute of Illinois, Oak Lawn, IL; ${ }^{6}$ Tennessee Women's Care, Nashville, TN; ${ }^{7}$ WomanCare, Arlington Heights, IL

(Presented by: James Lukban, DO)

Introduction and Objectives: Women with anatomic stress urinary incontinence may also have a measure of bladder neck funneling, with ultrasound confirmation of its presence found to be associated with a lower maximal urethral closure pressure, a smaller area under the urethral pressure profile curve, a lower leak point pressure and a larger volume of leakage on pad testing. Radiofrequency (RF) collagen denaturation has been found to reduce tissue compliance, with a potential role in reducing bladder neck funneling in patients with stress urinary incontinence (SUI). We sought to determine the efficacy of RF employing the Lyrette device (Verathon Inc., Bothell, Washington, USA) in women with mild to moderate SUI.

Methods: On-going, prospective, 36-month, open-label, multi-center clinical study enrolling women with anatomic SUI and urodynamic indices of Valsalva Leak-point Pressure (VLPP) $>90 \mathrm{~cm} \mathrm{H2O}$ and Maximum Urethral Closure Pressure $(\mathrm{MUCP})>45 \mathrm{~cm} \mathrm{H} 2 \mathrm{O}$. Intervention was in the form of transurethral RF energy delivered to the bladder neck and proximal urethra at 65 degrees Celsius to a total of 36 foci. Primary endpoint was the percentage of patients deemed to be "dry" at 12 months as defined by $<1$ leak on three-day diary or $<1$ gram of leak on one-hour pad weight test (PWT). Secondary endpoints included the proportion of patients with $>50 \%$ decrease on PWT; cough stress test (CST); quality of life (QoL) measures including the Incontinence Quality of Life (I-OOL) Ouestionnaire and Urogenital distress Inventory (UDI-6); immediate post-treatment pain as per the Visual Analog Scale (VAS) (1 minimum -10 maximum); and patient satisfaction.

Results: Fifty-two patients underwent treatment and had follow-up through 12 months. Subjects were analyzed by Intent-to-Treat. Dry rate was $82 \% ; 73 \%$ had a $>50 \%$ decrease on PWT; and $71 \%$ exhibited a negative CST. Seventy-nine percent had an improvement in I-OoL, with significant improvement $(\mathrm{P}<0.0001)$ observed in mean UDI-6 scores. No significant post-treatment pain was recorded, with a mean VAS score of $1.7+1.8$. Seventy-four percent were "very" or "somewhat" satisfied and 88\% would "refer this treatment to a friend". No serious adverse events were recorded.

Conclusions: At 12 months, treatment of women with mild to moderate SUI employing Lyrette yielded favorable efficacy, improvement in OoL and high patient satisfaction. Funding: Verathon Inc., Bothell, Washington, USA

\section{Poster \# NM41}

\section{PATHOLOGIC OUTCOMES FOLLOWING URETHRAL DIVERTICULECTOMY IN WOMEN}

Melissa Laudano, $\mathrm{MD}^{1}$, Asha Jamzadeh, $\mathrm{BS}^{2}$, Dean Elterman, $M^{2}$, Stephan Seklehner, $M^{2}$, Richard Lee, $\mathrm{MD}, \mathrm{MBA}^{2}$, Renuka Tyagi, $\mathrm{MD}^{2}$, Brian Robinson, $\mathrm{MD}^{4}$, Steven Kaplan, $\mathrm{MD}^{2}$, Alexis Te, $\mathbf{M D}^{2}$ and Bilal Chughtai, $\mathbf{M D}^{2}$

${ }^{1}$ Department of Urology, Weill Medical College of Cornell University, New York, NY; ${ }^{2}$ Department of Urology, Weill Medical College of Cornell University, New York-Presbyterian Hospital, New York, NY; ${ }^{3}$ Department of Urology,

Landesklinikum Baden-Mödling, Baden, Austria; ${ }^{4}$ Department of Pathology \& Laboratory Medicine, Weill Medical College of Cornell University, New York-Presbyterian Hospital, New York, NY

(Presented by: Melissa Laudano, MD)

Introduction and Objectives: Although most urethral diverticula in women are benign, there is a subset of patients who develop malignant changes. Limited studies report the pathologic findings associated with this relatively rare entity. In this study, we describe the clinicopathologic findings of women who underwent urethral diverticulectomy.

Methods: A consecutive series of 29 women who underwent surgical resection of a urethral diverticulum were identified between 1992 and 2013. Clinical and radiographic data was collected by retrospective review of patient medical records. All pathological slides were re-reviewed by a single urologic pathologist.

Results: The mean age of women was $44.7( \pm 14.5)$ years. Of the 14 women with clinical data, nine $(64 \%)$ presented with urgency, seven (50\%) with urinary frequency, three (21\%) with urinary incontinence and three (21\%) with dysuria. Mean diverticular size, measured as the greatest diverticular dimension radiographically, was $2.3( \pm 1.4) \mathrm{cm}$. Although one patient (3\%) had invasive adenocarcinoma on final pathology, the remaining 28 cases (97\%) demonstrated benign features. The most common findings were inflammation (55\%) and nephrogenic adenoma (21\%). One woman (3\%) had a leiomyoma associated with the diverticulum, and seven (24\%) had no significant pathologic changes aside from the diverticulum itself (See Table). 
Conclusions: Limited data exist regarding the rates of various clinicopathologic findings associated with urethral diverticula. Although most urethral diverticula in women are benign, there is a subset of patients who develop malignancy in association with the diverticulum. In this series, $97 \%$ of cases had a benign histology. These findings are important when counseling patients regarding treatment options.

\begin{tabular}{|c|c|c|}
\hline & 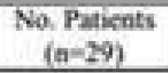 & $\%$ \\
\hline \multicolumn{3}{|l|}{ Type of tining } \\
\hline Squamous cpithalium & 10 & $34 \%$ \\
\hline Urothelinm & 9 & II: \\
\hline Mod unothethal ind squimous & 7 & 24 \\
\hline No lining identificd & 2 & $7 \%$ \\
\hline Ficat nal metaplain & 1 & $3 \%$ \\
\hline \multicolumn{3}{|l|}{ Pathologle findiage } \\
\hline Fiterminition & 16 & 35 \\
\hline Chronik & 12 & $41 \%$ \\
\hline Acuise and cluboic & 4 & $14 \%$ \\
\hline Noned Jnemurkable & 7 & $24 \%$ \\
\hline Nophrogenie adenoma & 6 & 2I\% \\
\hline Srombl calcilisation & 4 & $14 \%$ \\
\hline Ulecration & 3 & 107 \\
\hline Leiomyoma & $\mathrm{I}$ & $3 \%$ \\
\hline Tavasve denochretnans & 1 & 37 \\
\hline
\end{tabular}

\section{Poster \# NM42}

IMPACT OF TRANSDERMAL OXYBUTYNIN (OXYTROL) ON OUALITY-OF-LIFE IN WOMEN WITH OVERACTIVE BLADDER: A POST HOC, POOLED ANALYSIS OF TWO PLACEBOCONTROLLED TRIALS

Shuchean Chien, Krishna Jhaveri, Alankar Gupta and Rajesh Mishra

(Presented by: Shuchean Chien)

Introduction and Objective: Safety and effectiveness of transdermal oxybutynin (Oxytrol) has been thoroughly assessed and clinically proven. However, direct evidence of patient's healthrelated quality of life (HROoL) improvement as related to clinical efficacy has not been established. This analysis assesses the correlations between HROoL and Oxytrol treatment efficacy in women with OAB.

Methods: Data from two randomized, double-blind, placebocontrolled trials in 453 women with OAB were pooled for the analyses. Patients received Oxytrol or placebo for 12 weeks while underwent a simultaneous behavioral therapy. Patient's average urinary frequency (UF), urinary incontinence (UI) and urinary volume (UV) were measured based on voiding diary. HROoL was self-rated using a validated Incontinence Impact Questionnaire (IIO), which assesses emotional wellbeing, physical, social and travel functionality/quality. Treatment benefits of each of the four endpoint measures (UI, UF, UV and total IIO scores) were ranked as no improvement, marginal, minimal, moderate, substantial and complete recovery based on percent change from baseline (\%CFB). Patient's HROoL improvement (\%CFB of total IIO scores) and Oxytrol treatment efficacy (\%CFB of UI, UF and UV) are considered well correlated if $|r| \geq 0.3$ and $p \leq 0.05$.

Results: Patients in both treatment groups had similar demographics, baseline OAB and HROoL conditions. Significantly greater treatment benefits were observed in women treated with Oxytrol $(\mathrm{N}=223)$, as compared to those in placebo $(\mathrm{N}=230)$. Greater percentage $(\%)$ of women treated with Oxytrol had moderate, substantial or full improvement $(\%$ CFB: $\geq 30 \%$ to $100 \%)$ in UI ( $81 \%$ vs. $73 \%, p<0.01$ ), UV (39\% vs. $20 \%, p<0.0001$ ), HROoL ( $64 \%$ vs. $52 \%, p<0.02$ ); and a less significant difference in UF ( $24 \%$ vs. $21 \%, p=0.07)$. Patient's HROoL improvement correlated well with UI and UF reduction (UI: $r=0.390, p<0.0001$; UF: $r=0.300, p<0.0001$ ); whereas the correlation with the UV increase was significant but marginal $(\mathrm{r}=0.121, \mathrm{p}=0.02)$.

Conclusions: In these pooled placebo controlled trials, the observed $\mathrm{OAB}$ symptom reduction correlated well with patient's self-assessed improvement in HROoL. This underscores the clinical benefit of Oxytrol treatment on quality of life being related to effective symptom control.

Clinical Relevance: Female Urology-including Incontinence

\section{Poster \# NM43}

\section{EFFICACY OF BULKING AGENTS IN PATIENTS WITH PERSISTENT LEAKAGE AFTER A MID-URETHRAL SLING.}

Luis Espaillat-Rijo, $\mathrm{MD}^{1}$, Orawee Chinthakanan, $\mathrm{MD}^{2}$, Vivian Aguilar, $\mathbf{M D}^{2}$ and G. Willy Davila, $\mathbf{M D}^{2}$

${ }^{1}$ Cleveland Clinic Florida; ${ }^{2}$ Cleveland Clinic Florida, Weston, FL (Presented by: Luis Espaillat-Rijo, MD)

Objective: To assess the efficacy of bulking agents [Macroplastique $(\mathbb{C}$ (MPO), Uroplasty, Minneapolis, USA] in patients with persistent leakage after mid-urethral slings.

Introduction: Mid-urethral tapes/slings are used in the treatment of stress urinary incontinence (SUI) with high success rates. They have replaced the Burch colposuspension as the gold standard for SUI treatment. But what happens to those patients who have persistent leakage? Repeat slings and office therapy bulking agents have been common treatment options however only scant data is available describing their outcomes.

Methods: IRB approval was obtained and the institutional Urogynecologic database was queried to identify patients who were treated with MPO after having been previously treated with a suburethral sling. Demographic information, surgical history, urodynamics parameters, associated morbidity, subjective and objective data were collected and analyzed. The primary outcome was patient reported improvement on a standardized, validated improvement satisfaction questionnaire (ISS), and success was defined as greatly improved or cured at the patient's last follow-up visit. Data was analyzed by the second author using chi-square test for categorical data, and t-test for continuous data.

Results: A total of 43 patients were identified as having had a failed sling and subsequently underwent MPO injections in the office, three were lost to follow-up. The mean age was $70.3 \pm 11.42$ years, the mean follow-up time after the first MPO injection was $48.5 \pm 47.7$ weeks and the median number of injections was one (range 1-10). Mean volume of MPO injected per session was $3.8 \mathrm{ml}$. (range 2.5-6). At their last visit, 28 (70\%) reported improvement and 12 (30\%) did not improve (Table 1). Hypermobility was a statistically significant negative predictor for subjective success with MPQ, in line with available literature.

Conclusion: Bulking agents may be beneficial in patients with prior sling failure. Patients with ISD and no hypermobility are most likely to benefit. More research is needed to determine individual parameters associated with this beneficial effect. 
Clinical Relevance: Female Urology-including Incontinence

$\begin{array}{ccc}\text { Cured or Improved } & 28 & 70 \\ \text { Not improved } & 12 & 30\end{array}$

\section{Poster \# NM44}

\section{CONCURRENT EXCISION OF MID-URETHRAL SLING DURING REPEAT MID-URETHRAL SLING IS NOT ASSOCIATED WITH CONTINENCE OUTCOMES}

Robert Chan, $\mathrm{MD}^{1}$, Jason Scovell, $\mathrm{BS}^{2}$, William Johnson, $\mathrm{BA}^{2}$, Rose Khavari, $\mathrm{MD}^{1}$ and Danielle Antosh, $\mathrm{MD}^{3}$

${ }^{1}$ Department of Urology, Houston Methodist Hospital, Houston, TX; ${ }^{2}$ Baylor College of Medicine, Houston, TX; ${ }^{3}$ Department of Obstetrics and Gyncology, Houston Methodist Hospital,

Houston, TX

(Presented by: Robert Chan, MD)

Introduction: The role of excision prior to a repeat midurethral synthetic sling for recurrent or persistent stress urinary incontinence is unknown. The purpose of our study was to evaluate if excision of a prior sling during repeat midurethral sling placement is associated with greater success or failure.

Methods: Retrospective chart review was performed for all women who had undergone a sling procedure at our institution between 2009 and 2013. We identified patients receiving repeat mid-urethral slings and evaluated outcome by quantifying the number of pads used before and after surgery. Cure was defined as no pads, improvement as less pads and failure as the same or increased amount of pads. Success was defined as either cure or improvement. Success and failure were evaluated for women who had their prior sling excised or left in place. Medians are reported, and Mann-Whitney was calculated for significance $(p<.05)$.

Results: 30 women with prior mid-urethral sling underwent a repeat mid-urethral sling for primary failure or recurrence. 17 initial slings were excised (none for extrusion or erosion), and 13 were left in place. There was no difference in success between women who had their sling excised or left in place (88.2\% vs. $100 \%, p=0.49)$, and cure rates were similar $(76.5 \%$ vs. $76.9, p=1.00)$. Women whose sling was excised or left in place were similar in age (56.3 y vs. $64.5 \mathrm{y}, \mathrm{p}=0.08$ ) and follow-up (15.6 w vs. $16.8 \mathrm{w}, \mathrm{p}=0.60$ ), but women with sling excision had a lower BMI (24.6 vs. 29.2, $\mathrm{p}=0.02$ ).

Conclusion: Excision was not associated with improved success in women who underwent a repeat mid-urethral sling. Clinical Relevance: LUTS / Voiding Dysfunction

\section{Poster \# NM45}

COMPARISON OF SUCCESS AND COMPLICATION RATES OF THE DISTAL URETHRAL POLYPROPYLENE SLING (DUPS) AND THE MINIARC SLING FOR FEMALE STRESS INCONTINENCE

Anthony Dyer, MD, Arthi Satyanarayan, BA, Joel Funk, MD and Christian Twiss, MD

University of Arizona College of Medicine, Tucson, AZ (Presented by: Anthony Dyer, MD)

Objective: To retrospectively compare outcomes and postoperative complications between the retropubic Distal Ure- thral Polypropylene sling (DUPS) and MiniArc sling for treatment of female stress urinary incontinence (SUI).

Methods: Charts were retrospectively reviewed and 119 patients who underwent a DUPS or MiniArc sling by a single surgeon (C.T.) with a minimum of six months follow-up were identified. Surgical complications were recorded. Patient selfreported SUI outcomes were assessed by the validated Incontinence Symptom Severity Index (ISS) and the Urogenital Distress Inventory-Short Form (UDI-6). Self-reported success was defined as none or mild SUI symptoms or bother on the ISS and UDI-6, respectively. Self-reported pad use was also used as a separate indicator of success. When assessing pad use, success was defined as 0-1 pad per day (ppd) with no history of subsequent SUI intervention. Continuous data was analyzed by 2-tailed t-test and categorical data by 2-tailed Fischer's exact test.

Results: Of the 119 patients, 55 and 64 patients underwent DUPS and MiniArc sling, respectively. Mean follow-up for the entire cohort was 12.9 months, 15.6 months DUPS vs. 10.5 months MiniArc $(p<0.001)$, and the mean ages were 60.3 years DUPS vs. 61.8 years MiniArc $(p=0.61)$. No significant difference was found in vaginal deliveries, concurrent pelvic surgeries, baseline pad use or baseline self-reported symptom severity. Overall success was $93.3 \%$ when assessed by self-reported pad use; $96.4 \%$ (53/55) DUPS vs. 93.3\% (58/64) MiniArc $(p=0.28)$. Mean postoperative ppd was 0.22 ppd DUPS vs. $0.72 \mathrm{ppd}$ MiniArc $(p=0.06)$, and the percentage reduction in ppd was 91.5\% DUPS vs. $79.6 \%$ MiniArc $(p=0.09)$. Overall success as assessed by the ISS was $84 \%(42 / 50) ; 85.7 \%(18 / 21)$ DUPS vs. $82.7 \%(24 / 29)$ MiniArc $(p=1.0)$. Overall success as assessed by UDI-6 was also $84 \%$ (42/50); 80.9\% (17/21) DUPS vs. 86.2\% (25/ 29) MiniArc $(p=0.71)$. Postoperative urinary retention requiring intervention was 5.9\% (7/119) for the entire cohort; $9.1 \%$ (5/ 55) DUPS vs. 3.1\% (2/64) MiniArc $(p=0.25)$. Other complications related to sling surgery were $8.4 \%(10 / 119)$ for the entire cohort; $14.6 \%$ (8/55) DUPS vs. 3.1\% (2/64) MiniArc ( $p=0.04)$.

Conclusions: The DUPS and MiniArc urethral slings have similar success rates and overall low complication rates. However, the DUPS was noted to have higher complication rates and a trend toward higher rates of urinary retention as compared to the MiniArc sling.

Clinical Relevance: Female Urology-including Incontinence

\section{Poster \# NM46}

THE MEDICOLEGAL AND ECONOMIC IMPLICATIONS OF FDA COMMUNICATIONS REGARDING THE USE OF TRANSVAGINAL MESH

Marc Colaco, MD, MBA, Austin Hester, MD, Jason Sandberg, MD, Jayadev Mettu, MD and Gopal Badlani, MD

Department of Urology, Wake Forest Health Sciences, WinstonSalem, NC

(Presented by: Marc Colaco, MD, MBA)

Introduction and Objectives: Although the use of transvaginal mesh placement has long been considered an option for the treatment of pelvic organ prolapse (POP), recent developments have brought this practice under controversy. Several studies have questioned the efficacy and safety of mesh usage, and the FDA has made multiple communications regarding the potential complications of mesh usage. While the implications for clinical practice are still hotly debated, these developments also have medicolegal and economic implications. Thus, the purpose of this study is to analyze the product liability 
litigation rate for two large mesh manufactures and to correlate these findings to FDA communications as well as other possibly related factors.

Methods: Retrospective analysis was performed using legal case listings from the New Jersey judiciary Multicounty Litigation Center regarding mass tort for all pelvic mesh related cases filed prior to August 2013. This database was chosen as both Bard, Inc. and Ethicon Endo-Surgery, Inc. are based in New Jersey and thus New Jersey is the jurisdiction for these mass torts. Data was then compared to FDA communication releases and any landmark legal findings to determine correlation. Statistics were analyzed via parametric methods using Statsplus ${ }^{\circledR}$ software.

Results: A total of 4,385 Ethicon cases and 811 Bard cases were identified in this review. Rates of case filing did not significantly correlate to either FDA communication. Significant increases in filing were seen, however, in correlation with major verdicts decided in plaintiffs' favors: mean number of cases filed within three months of either landmark court case was significantly higher than at all other times during this period (273 versus 67, $\mathrm{p}<0.01$ ).

Conclusion: Device related lawsuits are an unfortunate reality of modern medicine, and awareness of liability is vital to the medical practice. Our results show that in regards to pelvic mesh related lawsuits, case filings are more related to court decisions than to FDA communications. These findings may reflect the impact of financial incentives within the medicolegal arena and demonstrate the need for physicians to keep abreast of current and ongoing legal precedence.

Clinical Relevance: Pelvic Organ Prolapse

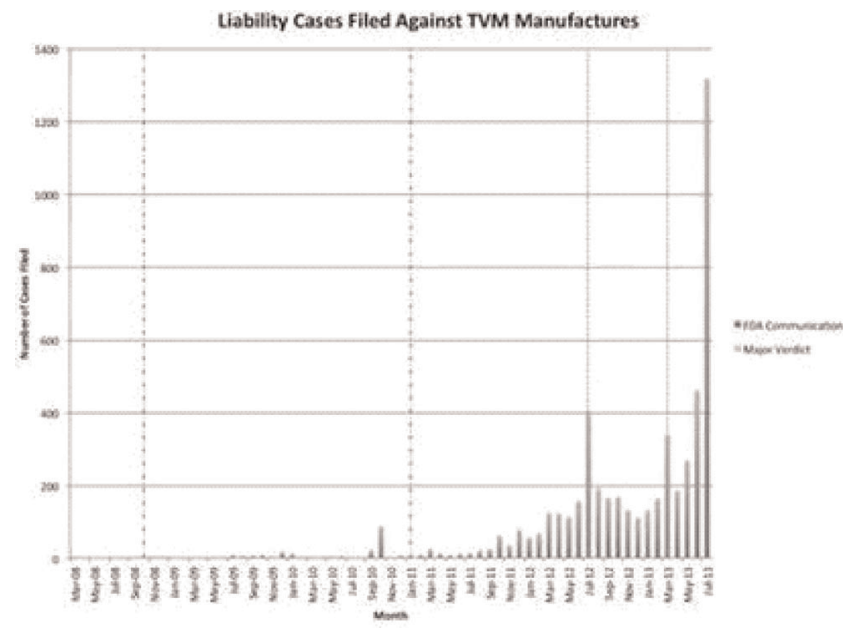

Poster \# NM47

PESSARY USE FOR SYMPTOMATIC PELVIC ORGAN PROLAPSE (SYMP POP) IN PATIENTS $\leq 65$ YEARS: FEASIBILITY RETROSPECTIVE STUDY

Constanza Ralph, $\mathrm{MD}^{1,2}$, Simone Pohlhammer, $\mathrm{MD}^{3}$, Bernardita Blümel, $\mathrm{MD}^{3}$ and Javier Pizarro-Berdichevsky, $\mathrm{MD}^{1,2,4}$

${ }^{1}$ Hosp. Dr. Sotero del Rio; ${ }^{2}$ Division de Obstetricia y Ginecologia, Facultad de Medicina, Pontificia Univ. Catolica de Chile; ${ }^{3}$ Hosp. Dr. Sotero del Rio, Santiago, Chile; ${ }^{4}$ Glickman Urology Institute, Cleveland Clinic, Clevaland, Ohio

(Presented by: Javier Pizarro-Berdichevsky, MD)

Introduction: Pessaries for symp POP could be offered as first line treatment option. Minimal data exist on its use in patients' $\leq 65$ years. Our aim is to evaluate the pessary feasibility management in this subgroup of patients.

Methods: Retrospective cohort study of patients with symp POP $\leq 65$ years managed with pessaries at the urogynecology unit of Dr. Sotero del Rio, public hospital in Santiago, Chile between July 2009 and June 2013. Inclusion criteria were $\leq 65$ years with symp POP $\geq$ stage II who underwent pessary fitting. Demographic, clinical characteristics and follow-up (FU) were analyzed. Patient Global Improvement Index (PGI-I) was used as a subjective outcome. The study protocol was approved by our institution's IRB.

Results: In the period, 49 of 179 patients (27.3\%) who underwent pessary fitting were $\leq 65$ years. In 39 of the 49 (79.5\%) the fitting was successful, but only 26 (66\%) finally used the pessary. Demographic and FU are described in Table 1. In the group that used the pessary, 13 (50\%) were sexually active. 13 patients $(50 \%)$ reported adverse effects, vaginal discharge was the most frequent $(69 \%)-6(23 \%)$ abandoned the treatment for several reasons (vaginal discharge, bleeding or pessary expulsion sensation). The PGI-I results were available in 17 of 26 patients (65\%). 15 (88\%) of those patients noted subjective improvement. In an analysis of worst-case scenario where all the patients who did not respond worsen, subjective improvement rate would be $57 \%$.

Conclusions: Pessary use in patients $\leq 65$ years could be an option as a first step therapeutic approach. After nine weeks of follow up (and up to 90 weeks), 76.9\% of the patients that used the pessary were still under this treatment. $88 \%$ of the patient's report subjective improvement. Therefore, pessaries use should not be restricted only to patients $>65$ years as in the most prevalent current practice. Prospective studies with higher sample size are needed to evaluate this subgroup, aiming to reduce possible bias intrinsic to a retrospective cohort.

Clinical Relevance: Pelvic Organ Prolapse

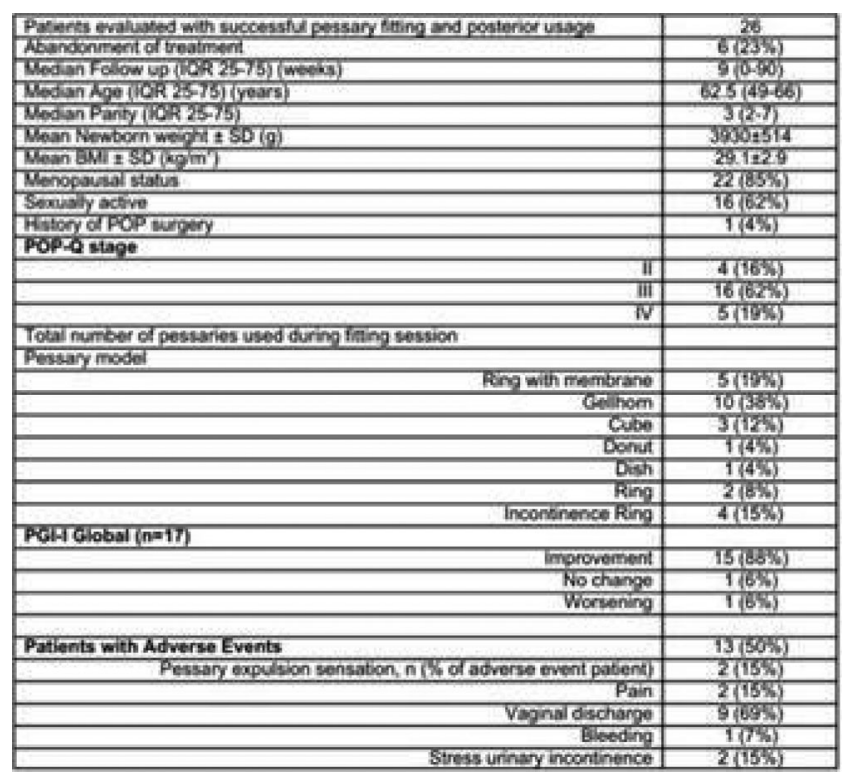

\section{Poster \# NM48}

\section{VALIDATION OF A PORCINE SIMULATOR FOR ENDOSCOPIC INJECTION OF A URETHRAL BULKING AGENT}

Tandis Soltani, Jessica Hammett, MD, Claudia Perez, Rebecca Do, Hanul Choi, Mina Samaan and Gamal Ghoniem Department of Urology, University of California, Irvine (Presented by: Jessica Hammett, MD) 
Introduction: Endoscopic injection of a urethral bulking agent (UBA) is a widely utilized treatment option for stress urinary incontinence. Acquiring an effective technique for UBA injection prior to patient application is a cost effective and patient centered objective that can be achieved with the use of simulators to augment trainee education. In this study, we aim to demonstrate face validity for a porcine simulator of endoscopic UBA injection.

Methods: Porcine bladders were mounted onto a modified hysteroscopy training model and a total of $1 \mathrm{~mL}$ of a surgical lubricant and methylene blue mixture was injected into the 3 , 6 , and 9 o'clock positions of the proximal urethra using a 22French injectable cystoscope. Pictures were taken prior to and after injection to compare coaptation, and following injection the bladder and urethra were dissected to determine the position of the UBA. An expert in female pelvic medicine graded participants on surgical technique and coaptation of the urethra on a scale of 1 to 5. Additionally, participants were timed from procedure start to finish.

Results: Three female pelvic medicine fellows and four urology residents participated in this study. The fellow's technique was rated significantly higher, 4.167 , than the residents, 3.125 $(p=0.0215)$. The coaptation score was higher for the fellow's, 4.5, compared to the residents, 3.625, and approached but did not reach significance $(p=0.065)$. Similarly, the amount of time from procedure start to finish approached but did not reach statistical significance with the fellows requiring an average of four minutes and the residents requiring an average of 3.125 minutes $(p=0.655)$. All participants graded the model as valuable and authentic.

Conclusion: Endoscopic injection of UBA can be effectively simulated with porcine bladders. Furthermore, it provides a unique opportunity to evaluate a trainee's technique and skill level prior to the operating room. Future research into the porcine simulator of endoscopic UBA injection is needed with larger participant numbers to further validate the simulator. Funding: No financial funding was received for this abstract.

\section{Poster \# NM49}

\section{ANALYSIS OF LUTS AND DYSPAREUNIA IN PATIENTS UNDERGOING TRANSVAGINAL MESH REMOVAL FOR MESH EXPOSURE AND EROSION}

Lara MacLachlan, MD, Justin Ellett, MD, Kelly Johnson, MD, Gini Ikwuezunma, MD, Michelle Koski, MD, Ross Rames, MD and Eric Rovner, MD

Charleston, SC

(Presented by: Lara MacLachlan, MD)

Introduction and Objectives: Transvaginal mesh has been commonly used in the operative management of stress urinary incontinence (SUI) and pelvic organ prolapse. Vaginal mesh exposure and mesh erosion into the urinary tract is a complication seen with these surgeries. We reviewed our experience with lower urinary tract symptoms (LUTS) and dyspareunia outcomes following mesh explantation over a seven-year period in those patients who underwent surgery for mesh removal.

Methods: A retrospective review of patients who underwent explantation of vaginal mesh from 2007 to 2013 was conducted. A total of 191 patients were identified of which 77 patients had evidence of vaginal mesh exposure and/or mesh erosion into the urinary tract. Other indications for mesh explantation included bladder outlet obstruction, pain and dyspareunia. The following symptoms were evaluated at baseline and at three months follow-up: irritative symptoms (frequency, urgency and nocturia = IS), obstructive symptoms (hesitancy, straining, incomplete emptying and positional voiding $=$ OS), dyspareunia, urge urinary incontinence (UUI) and SUI.

Results: The most common presenting symptoms for the entire cohort were IS (76.6\%) and OS (76.5\%). Of the cohort, 53 patients had vaginal mesh exposure, 19 had mesh erosion into the urinary tract and five had both. Patients presenting with vaginal mesh exposure did not differ in the array of presenting symptoms as compared with those patients with mesh erosion. The group of patients who had neither mesh exposure nor erosion had a significant improvement of all their symptoms at three months $(p<0.03)$. Of the patients with vaginal mesh exposure, there was a statistical improvement in all of their symptoms, with the exception of SUI. The patients with mesh erosion had a significant improvement in OS $(p<0.001)$ and dyspareunia ( $p=0.008$ ), but no improvement in IS, UUI, or SUI. Conclusions: This study demonstrates improvement in symptoms for those patients undergoing mesh removal surgery who do not have mesh exposure or erosion. However, for those patients with mesh erosion into the urinary tract there is comparatively less success in improving their urinary symptoms.

Clinical Relevance: Female Urology-including Incontinence

\begin{tabular}{lccc} 
& $\begin{array}{c}\text { No Mesh } \\
\text { Exposure or } \\
\text { Erosion } \\
(\mathrm{n}=113)\end{array}$ & $\begin{array}{c}\text { Mesh Exposure } \\
(\mathrm{n}=53)\end{array}$ & $\begin{array}{c}\text { Mesh Erosion } \\
(\mathrm{n}=\mathbf{2 4})\end{array}$ \\
\hline $\begin{array}{c}\text { Presenting Symptoms: } \\
\text { Irritative }\end{array}$ & $97(85.8 \%)$ & $40(75.5 \%)$ & $19(79.2 \%)$ \\
Obstructive & $78(69.0 \%)$ & $21(39.6 \%)$ & $11(45.8 \%)$ \\
Dyspareunia & $41(36.3 \%)$ & $36(67.9 \%)$ & $14(58.3 \%)$ \\
UUI & $66(58.4 \%)$ & $31(58.5 \%)$ & $14(58.3 \%)$ \\
SUI & $49(43.4 \%)$ & $28(52.8 \%)$ & $12(50.0 \%)$ \\
3 Months F/U: & & & \\
Irritative & $67(59.3 \%)$ & $24(45.3 \%)$ & $14(58.3 \%)$ \\
Obstructive & $29(25.7 \%)$ & $5(9.4 \%)$ & $5(20.8 \%)$ \\
Dyspareunia & $12(10.6 \%)$ & $8(15.1 \%)$ & $6(25.0 \%)$ \\
UUI & $45(39.8 \%)$ & $17(32.1 \%)$ & $15(62.5 \%)$ \\
SUI & $49(43.4 \%)$ & $28(52.8 \%)$ & $12(50.0 \%)$ \\
& & &
\end{tabular}

\section{Poster \# NM50} RECRUITMENT AND COST-EFFECTIVENESS OUTCOMES OF A
MASS MAILING RCT FOR PREVENTION OF URINARY INCONTINENCE

Diane Newman, DNP, ANP-BC ${ }^{1}$, Keri Kirk, $M^{2}$, Rebecca Kimmel, $\mathrm{BA}^{3}$, Janis Miller, $\mathrm{PhD}^{2}$, MaryAnn DiCamillo, BSN ${ }^{3}$, Marty Davis-Merritts, BA $^{2}$, K Rentenbach, $\mathbf{M S N}^{2}$, Mary Wang, $\mathbf{M S N}^{3}$, Kristy Keyock, $\mathbf{M S N}^{3}$ and Carolyn Sampselle, $\mathrm{PhD}^{2}$

${ }^{1}$ Urology, University of Pennsylvania, Philadelphia, PA;

${ }^{2}$ University of Michigan; ${ }^{3}$ University of Pennsylvania

(Presented by: Diane Newman, DNP, ANP-BC)

Introduction and Objectives: The Translating Unique Learning for Incontinence Prevention study enrolled women to determine whether a group class or a take-home DVD of behavioral techniques is more effective in primary prevention of urinary incontinence (UI). A reactive mass mailing recruitment was used for cost-effectiveness. Objectives were to examine the success of a telephone pre-screening method for potential enrollment in a primary prevention study, to determine the cost-effectiveness of mailings and to examine potential barriers.

Methods: Recruitment was via mass mailings broadly distributed using a commercial mailing list (50,000/site). An invitation letter was mailed to ethnically and racially diverse 
residents. A reactive approach was used. Woman was subsequently contacted by telephone for pre-screening.

Results: Nine mass mailings of at least 8,000 pieces ( $N$ $=83,500$ ) were sent; 2,230 respondents were pre-screened by telephone. Participants were women $55+$ yo with no UI or mild UI symptoms. Forty-eight percent $(n=1066)$ of respondents passed the pre-screen survey (see Table 1).

Conclusions: Mailing produced a diverse sample of women (see Figure 1) that were screened at a baseline evaluation visit. Mass mailings were found to be a cost-effective manner in which to recruit women for a primary prevention study.

Table 1: Top reasons for Telephone Pre-screening Failure

Southeast Michigan site: Reasons for Pre-screen Failure $(\mathrm{n}=1090)$

ICIO too high: 207 (32\%)

No longer Interested in participating: 131 (20\%)

No longer interested:125 (19\%)

Previous treatment for UI: 81 (12\%)

Bladder control medications: 28 (4\%)

Philadelphia, Pennsylvania site: Reasons for Pre-screen Failure $(n=1040)$

ICIO too high: 177 (36\%)

No longer Interested in participating: 75 (15\%)

Previous treatment for UI: 46 (9\%)

Not 55 years old: 44 (9\%)

History of neurologic disease: $32(6 \%)$

Compared to literature, expected recruitment response was $1 \%$ to $3 \%$. (see Table 2 ).

Table 2: Mailing Costs Per Participant

Southeast Michigan (42,000)

Mailing Costs

Cost/participant USD11.68

Philadelphia $(41,500)$

$\$ 16,500 \$ 20,412$

USD18.00/USD15.86**

\section{Poster \# NM51}

\section{URINARY RETENTION PREDICTS FAILURE TO VOID FOR} URODYNAMICS

Daniel Swanson, $\mathbf{M D}^{1}$ and Kamran Sajadi, $\mathbf{M D}^{2}$

${ }^{1}$ OHSU Department of Urology, Portland, Oregon; ${ }^{2}$ Portland, Oregon

(Presented by: Daniel Swanson, MD)

Introduction and Objectives: Patients often cannot void during urodynamic pressure-flow studies. Failure to Void for Urodynamics (FTVU) is a diagnostic dilemma because there is no way to distinguish an acontractile detrusor due to the artificial nature of the study versus an underlying pathophysiologic process; however, pressure-flow studies are the gold standard for diagnosing lower urinary tract obstruction. We describe the prevalence of and identify risk factors for FTVU.

Methods: We performed a retrospective review of all adults undergoing multichannel urodynamics from January 2012 through July 2013, excluding those with spinal cord injury or myelomeningocele. FTVU was defined as inability to void with the urodynamic catheter in place and inability to generate any detectable rise in detrusor pressure for the pressure-flow study. Voiders were compared to FTVU with regards to prior lower urinary tract surgery, neurological and psychiatric comorbidities, lower urinary tract medications, urinary retention and maximal cystometric bladder capacity (MCC) during the filling cystometrogram. Nominal variables were compared with $\chi 2$ test and continuous variables were compared with two-sided Student's t-test.

Results: Of 130 patients (48\% male), 46 (35\%) were unable to void (FTVU), which was similar in men and women (33\% versus $37 \%, p=0.64)$. Of these, seven (18\%) were able to void after the urodynamic catheter was removed. Age was similar in those with FTVU ( $54 \pm 18$ years) versus voiders ( $57 \pm 16$ years, $p=0.82$ ). Patients with urinary retention $(25 \%)$ were more likely to have FTVU than patients without urinary retention (59\% versus $28 \%, p=0.001$ ). Patients with recent exposure to antimuscarinic medications were less likely to have FTVU (14\% versus $41 \%, p=0.004)$. Men with FTVU had higher MCC than voiders $(497 \pm 214 \mathrm{~mL}$ versus $357 \pm 163 \mathrm{~mL}, p=0.005)$, however, there was no such difference in women. FTVU was more frequent with a self-reported history of anxiety only in men, but not significantly (58\% versus $27 \%$ without anxiety, $\mathrm{p}=0.08)$. On multiple logistic regression, the only predictors of FTVU were retention (OR 3.2, 95\% CI 1.3-8.0) and antimuscarinic medication use (negative predictor, OR $0.2,95 \% \mathrm{CI}$ 0.1-0.8).

Conclusions: Patients with urinary retention are less likely to void for pressure-flow urodynamic studies. Strategies to increase their likelihood of voiding for the study or alternative methods to diagnose or exclude obstruction are needed.

Funding: None.

Clinical Relevance: LUTS / Voiding Dysfunction

\section{Poster \# NM52}

URINARY INCONTINENCE IN DEMENTIA PATIENTS IS CAUSED BY DETRUSOR OVERACTIVITY RATHER THAN COGNITIVE DYSFUNCTION

Myong Kim, $\mathrm{MD}^{1}$, Seong Jin Jeong, $\mathrm{MD}, \mathrm{PhD}^{2}$, Sung Yong Cho, $\mathrm{MD}, \mathrm{PhD}^{3}$, Jae-Seung Paick, $\mathrm{MD}, \mathrm{PhD}^{1}$ and Seung-June $\mathrm{Oh}, \mathrm{MD}, \mathrm{PhD}^{1}$

${ }^{1}$ Department of Urology, Seoul National University Hospital, Seoul, Korea; ${ }^{2}$ Seoul National University Bundang Hospital, Seongnam, Korea; ${ }^{3}$ SMG-SNU Boramae Medical Center, Seoul, Korea

(Presented by: Myong Kim, MD)

Objectives: It has been reported the incidence of urinary incontinence (UI) in dementia patients is high. However, there has been little information regarding urodynamic findings in dementia. It is also obscure if the UI was originated from cognitive dysfunction or neurologic deficit. We tried to find out the urodynamic findings and whether cognitive dysfunction may affect the UI.

Methods: We retrospectively reviewed the medical records and urodynamic study (UDS) results of dementia patients between October 2004 and June 2013. To identify risk factors for UI, we correlated the presence of UI with the UDS findings and the Mini Mental State Examination (MMSE) score, which is known as representing degree of cognitive dysfunction.

Results: We identified 61 verified dementia patients. Their mean age was $72.3( \pm 6.0, \mathrm{SD})$ years and 35 patients $(57.4 \%)$ were males. There were 25 vascular dementia (41.1\%), 20 Alzheimer's disease (32.8\%), 16 others (26.1\%) including parkinsonism-progression, trauma-induced and frontotemporal type. Median scale of Clinical Dementia Rating was 1.0 (range 0.5 to 3.0) and mean score of MMSE was 20.2 ( \pm 6.3 ). 41 patients (75.4\%) had urgency and 32 patients (52.5\%) had urgency UI. Filling cystometry showed normal in 27 patients (44.3\%) and detrusor overactivity (DO) in 34 patients (55.7\%). Multivariate logistic regression analysis revealed that low 
MMSE score $(\beta=1.368, p=0.024)$ was the only independent predictive factor for UI. Age $(p=0.168)$, gender $(p=0.600)$, DO $(p=0.187)$ or subtypes of dementia $(p=0.400)$ did not show any significance (Table).

Conclusions: Our data showed UI in dementia patients was mainly affected by the low MMSE score, suggesting their UI may be caused by cognitive dysfunction rather than DO.

\begin{tabular}{lcccccc}
\multicolumn{8}{l}{ Table. Predictive factors for UI (by multiple logistic regression) } \\
\hline & $\beta$ & p-value & OR & \multicolumn{2}{c}{$95 \% \mathrm{Cl}$} \\
\cline { 5 - 7 } \cline { 5 - 6 } & 0.833 & 0.168 & 2.300 & 0.704 & Upper \\
\hline Gender (fermale) & 0.351 & 0.600 & 1.420 & 0.382 & 5.272 \\
DO in UDS & 0.864 & 0.187 & 2.373 & 0.658 & 8.551 \\
MMSE score (<20) & 1.368 & 0.024 & 3.928 & 1.193 & 12.935 \\
Alzheimer's disease & -0.578 & 0.400 & 0.561 & 0.146 & 2.155 \\
\hline
\end{tabular}

\section{Poster \# NM53}

\section{DETRUSOR FORCE IN WOMEN. EVALUATION FROM} MATHEMATICAL MODELING OF PRESSURE-FLOW STUDIES

Françoise Valentini, MD, PhD, Pierre Nelson, $\mathrm{PhD}^{1}$, Philippe Zimmern, $\mathrm{MD}^{2}$ and Gilberte Robain, $\mathrm{MD}, \mathrm{PhD}^{1}$

${ }^{1}$ ER6-Université Pierre et Marie Curie (Paris 06) France;

${ }^{2}$ UTSouthwestern, Dallas TX

(Presented by: Françoise Valentini, MD, PhD)

Introduction and Objective: Evaluation of detrusor force in women is a difficult challenge. Our hypothesis was that female voidings were governed by similar parameters than male voidings: the detrusor force and a "urethral resistance". Our objectives were, using the VBN mathematical model of micturition (1) to analyze pressure-flow (P-F) data, to evaluate the detrusor force $(\mathrm{k})$ in women and to search for a correlation with a "urethral resistance" (U) simulated by an obstruction.

Methods: P-F studies of non-neurogenic women referred for evaluation of lower urinary tract dysfunction (LUTD) were retrospectively analyzed. Criteria for inclusion were P-F tracings providing maximum flow rate Omax and detrusor pressure at Omax (pdet.Omax) without significant contribution of abdominal pressure $(< \pm 3 \mathrm{~cm} \mathrm{H2O}$ between onset of flow and Omax), an initial bladder volume (Vini) $>100 \mathrm{~mL}$ and a non-interrupted flow. VBN simulations needed to know Vini and the urethral catheter diameter. Evaluated parameters were $\mathrm{k}$ (without unit) and $\mathrm{U}$ (unit $\mathrm{cm} \mathrm{H2O).} \mathrm{Standard} \mathrm{values} \mathrm{were}$ $\mathrm{k}=1.0$ and $\mathrm{U}=0$.

Results: The population comprised of 154 women (mean age $58.8 \pm 17.0 \mathrm{y})$. Two sub-groups were identified based on the feasibility of the standard VBN (A: 125 women, mean age $58.0 \pm 17.2 \mathrm{y}$ ) or not (B: 29 women, mean age $62.1 \pm 15.5 \mathrm{y}$ ). In sub-group $\mathrm{A}$, the VBN parameter $\mathrm{k}$ and $\mathrm{U}$ were identified. $\mathrm{k}$ range was [0.14-1.55] and U range [0.0-73.0 cm H2O] with a significant correlation $(p<.0001)$ between $k$ and $U$ (Fig): $\mathrm{k}=.259+.015^{*} \mathrm{U}(\mathrm{R} 2=.723)$. That result was independent of the main complaint. On the opposite, there was a great scattering when a correlation was search between $\mathrm{k}$ and age or maximum urethral closure pressure. Sub-group B was characterized by low pdet.Omax-high Omax (funnelling of both bladder neck and urethra?).

Conclusions: The VBN analysis can evaluate the detrusor force in women who void without major straining efforts with a non-interrupted flow. The detrusor force is smaller than in men [1] and the range less spread out. As in men, there is an adjustment of the detrusor force to compensate a "urethral resistance." Further study will be devoted to analyze the mechanisms underlying the voidings at low pressure.

\section{1- NAU 2000;19:153-76}

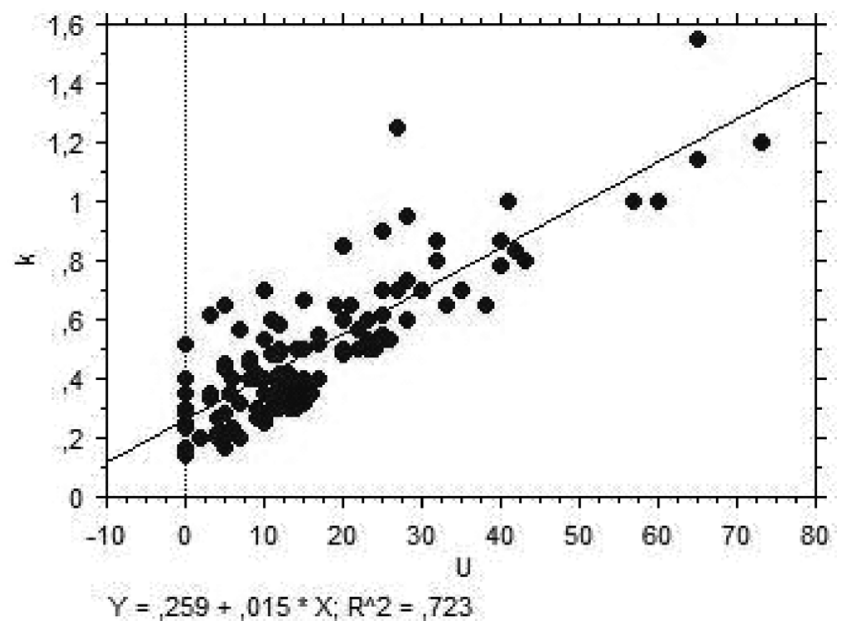

Poster \# NM54

USEFULNESS OF ICE WATER TEST (IWT) TO UNMASK DETRUSOR OVERACTIVITY (DO)

Karine Loiseau, MD, Françoise A Valentini, MD and Gilberte Robain, MD, PhD

Paris, France

(Presented by: Karine Loiseau, MD)

Introduction and Objective: Ice Water Test (IWT) is 56 years old. Initially, it was used to discriminate between upper (UMN) and lower motoneuron (LMN) lesions in patients with neurogenic urinary symptoms. (1) Recently it has been demonstrated as positive in various clinical conditions; and (2) Today IWT is usually performed to unmask detrusor overactivity (DO) when conventional cystomanometry (CCMM) is unable to give a reliable conclusion about the detrusor behavior. Our purpose was to retrospectively analyze IWT in patients whom CCMM was not contributory to assess detrusor behavior.

Methods: IWT of 125 patients were analyzed. Detrusor function was identified as normal (despite complaint of urgency), unspecified or underactive during CCMM. IWT was performed after CCMM. Starting from bladder empty, $250 \mathrm{ml}$ of saline at $\leq 4^{\circ} \mathrm{C}$ were instilled at $100 \mathrm{~mL} / \mathrm{min}$. Filling was followed by a waiting time of $1 \mathrm{~min}$. Pressures and flow rate were recorded during filling and waiting time (3). IWT was defined as positive if a non inhibited detrusor contraction (NIDC) $\geq 15 \mathrm{cmH2O}$ with leakage occurred within $3.5 \mathrm{~min}$ (filling plus waiting time), intermediate in case of NIDC without leakage and negative in case of absence of any detrusor contraction.

Results: The population consisted of 92 women ( $58.5 \pm 17.3 y)$ and 33 men $(56.5 \pm 14.2 y$. Main complaints were mixed or urge incontinence (48/125) and chronic retention (47/125). Eightyeight patients had a history of neurological disease. From CCMM, detrusor behavior was founded normal for 40 patients, unspecified for 68 and underactive for 17. IWT was positive for 16 patients and intermediate for seven. Patient's diagnosis is detailed in figure.

Conclusions: IWT allows unmasking DO in patients with history of neurological disease whose CCMM gave no reliable conclusion on the detrusor behavior. IWT is simple, easy to perform and not money consuming. Thus, IWT appears as a valuable complement of urodynamics and must be performed when the CCMM of a patient complaining of urgency leads to an ambiguous conclusion. In our results, we unmask 23/125 
(18.4\%) DO. A prospective study is necessary to confirm these findings.

References: 1. J Urol 1999; 162: 1890-6; 2. J Urol 2010;183:1686-92; 3. Br J Urol 1994; 73: 498-503.

Clinical Relevance: Neurogenic Bladder

\section{Patient's diagnosis with IWT (+)/(i)}

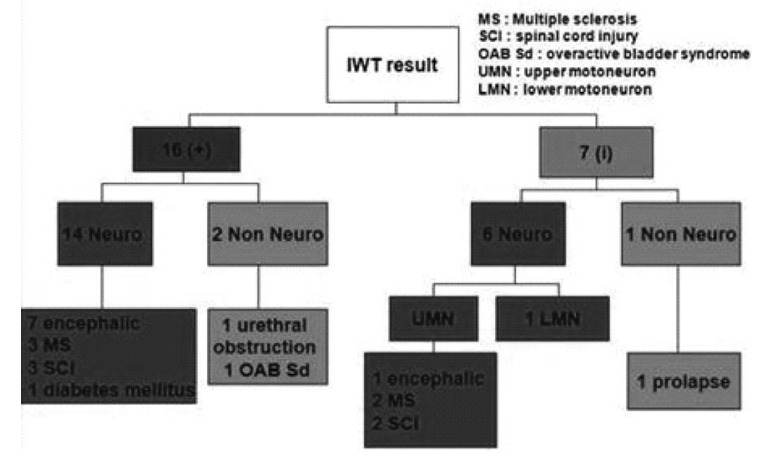

Poster \# NM55

URODYNAMIC PARAMETERS ASSOCIATED WITH THE RESOLUTION OF OVERACTIVE BLADDER SYMPTOMS AFTER ANTERIOR VAGINAL WALL PROLAPSE REPAIR

Chong Choe, $\mathrm{MD}^{1}$, Justina Tam, $\mathrm{BS}^{2}$, Eugene Lee, $\mathrm{MD}^{1}$, Kathleen Kobashi, $\mathbf{M D}^{1}$ and Alvaro Lucioni, $\mathbf{M D}^{1}$

${ }^{1}$ Virginia Mason Medical Center, Seattle, WA; ${ }^{2}$ Stony Brook

University School of Medicine, East Setauket, NY

(Presented by: Chong Choe, MD)

Objective: The purpose of this study was to determine which urodynamic (UDS) parameters were associated with the resolution of overactive bladder (OAB) symptoms after anterior pelvic organ prolapse (POP) repair.

Methods: All patients who underwent anterior POP repair with preoperative UDS evaluation and preoperative and postoperative Urogenital Distress Inventory (UDI-6) were eligible for the study. Two groups were stratified according to questions 1 and 2 of the UDI- 6 to assess for OAB symptoms. Both groups had OAB symptoms prior to POP repair. Postoperatively, group A were "not at all" bothered by OAB symptoms. Group B were "slightly," "moderately" or "greatly" bothered by OAB symptoms. The preoperative UDS parameters were then analyzed.

Results: 45 patients met our inclusion criteria. 12 patients (26.7\%) met criteria for group A and 33 patients (73.3\%) met criteria for group B. Group A had an average Baden-Walker grade of 2.23 (sd 0.93) and group B had an average BadenWalker grade of 2.21 (sd 0.90) for the anterior compartment. The average number of days the UDI- 6 questionnaires were completed after surgery for group A and B were 321 and 270 days, respectively. The average preoperative and postoperative combined score of questions 1 and 2 of the UDI- 6 for group A was 4.58 and 2.00, respectively. The average preoperative and postoperative combined score of questions 1 and 2 of the UDI-6 for group B was 5.15 and 4.58, respectively. The UDS parameter which was statistically different between group A and B was detrusor pressure (Pdet) at maximum flow (Omax) at 28.40 vs. $18.35 \mathrm{~cm} \mathrm{H2O}(\mathrm{p}<0.05)$, respectively.

Conclusions: The only UDS parameter associated with resolution of OAB symptoms after anterior POP repair was Pdet at Omax. We can speculate that this could be secondary to correction of anatomic obstruction after anterior POP repair. Further study is needed in a larger group of patients to confirm our findings.
Source of Funding: None.

Clinical Relevance: Urodynamics

\section{Poster \# NM57}

\section{URODYNAMICS: A POOR PREDICTOR OF REPEAT ONABOTULINUMTOXIN A INJECTION}

Dhruti Patel, MD, Elizabeth Ferry, MD, Anne Sammarco, MD, MPH, Sangeeta Mahajan, MD and Adonis Hijaz, MD Urology Institue, University Hospitals Case Medical Center, Cleveland, Ohio

(Presented by: Dhruti Patel, MD)

Introduction and Objectives: Urinary incontinence due to detrusor overactivity (DO) has multiple management strategies; one of which is intradetrusor injection of Onabotulinumtoxin A. It has been shown to be a highly effective treatment for refractory DO but little is known about how to predict response to injection and how long that response will last. Additionally, urodynamic parameters have evaluated change in bladder capacity and decreased detrusor pressure. However, these changes not been correlated to length of response. The objective is to evaluate the relationship of urodynamics parameters, time interval to repeat Onabotulinumtoxin A injection and number of repeat therapies required in adults.

Methods: A retrospective chart review was conducted in all patients who underwent Onabotulinumtoxin A detrusor injections for DO between January 1, 2008 and January 1, 2013. 53 charts were reviewed with respect to urodynamics, number of detrusor injections and time between detrusor injections.

Results: Peak amplitude of DO during urodynamics compared to time to re-injection was analyzed using linear regression and though not significant, did show a trend toward longer time to re-injection as peak amplitude of DO increased. Using a chi-square analysis to evaluate number of DO episodes during urodynamics and the need for re-injection $p=0.143$. Bladder capacity, volume at the start of DO and leakage during urodynamics were all not significant predictors of need for re-injection.

Conclusions: In a small cohort of patients with refractory detrusor overactivity who underwent Onabotulinumtoxin A injection for management, parameters of urodynamics were not able to predict need for re-injection, though number of episodes of DO during urodynamics did trend toward significance.

Financial Disclosure: None.

Clinical Relevance: LUTS / Voiding Dysfunction

\section{Poster \# NM58}

\section{SALVAGE TRANSOBTURATOR SLING PLACEMENT AFTER INITIAL FAILED SLING FOR MALE STRESS URINARY INCONTINENCE}

Edward Martinez, BS, Jack Zuckerman, MD, Brooke Edwards, MD, Katherine Henderson, BS and Kurt McCammon, MD

Eastern Virginia Medical School, Norfolk, VA

(Presented by: Jack Zuckerman, MD)

Objectives: To evaluate the outcome of patients treated with a salvage AdVance male sling after a failed primary transobturator sling placement.

Methods: Retrospective review of patients treated at our center with a primary and subsequent salvage AdVance sling. Success 
was defined as a dry safety pad or no pads (cured), or greater than $50 \%$ improvement in pads used per day and patient satisfaction (improved). Early primary sling failures $(<6$ months) were compared with late ( $\geq 6$ months) failures with regards to continence outcomes.

Results: We identified 18 patients who underwent a salvage AdVance sling at our institution. Overall success was $72 \%$ at six months post-op and $56 \%$ at a mean follow up of 17.5 months, including $50 \%$ and $39 \%$ of patients who were dry at those same time periods. Patients failing early following their primary sling $(n=10)$ enjoyed improved outcomes with salvage sling placement compared to patients who failed late $(n=8)$ following the primary sling. At six months, more patients in the late primary failure group were cured ( $75 \%$ vs $30 \%, p=0.031)$. These improved cure rates remained significant through final follow up with cure rates of $63 \%$ and $20 \%$, respectively $(p=0.041)$.

Conclusions: Salvage AdVance male sling is a viable treatment option after a failed primary sling procedure, especially in patients who demonstrated a prolonged efficacy period prior to primary sling failure.

Clinical Relevance: Male Incontinence

\section{Poster \# NM59}

\section{DURABLE OUTCOMES USING ADVANCE TRANSOBTURATOR} MALE SLINGS FOR MILD STRESS URINARY INCONTINENCE.

Jack Zuckerman, MD, Brooke Edwards, MD and Kurt McCammon, MD

Eastern Virginia Medical School, Norfolk, VA

(Presented by: Jack Zuckerman, MD)

Objectives: To evaluate our extended outcomes placing AdVance transobturator male slings in men with mild stress urinary incontinence (SUI).

Methods: Retrospective review of prospectively maintained database. We included men who received an AdVance sling for SUI and who used two pads per day or less preoperatively. Patients with less than 24 months of follow up were excluded. Success was defined as cured (no pads or a dry safety pad) or improved ( $>50 \%$ improvement in pad counts). All patients requiring secondary procedures were considered failures.

Results: Twenty-nine patients met our inclusion criteria and were included in the study. Median follow-up for the cohort was 44 months (mean 41 months, range 24-68 months). Overall success was $79 \%$, including $69 \%$ who were cured and $10 \%$ who were improved. Complications were similar to prior reports including urinary retention in $10 \%$ and scrotal pain and a UTI in one patient each. There were no delayed complications, including no mesh erosions or infections.

Conclusions: The AdVance transobturator male sling appears to have durable results in patients with mild incontinence without the development of delayed complications.

Clinical Relevance: Male Incontinence

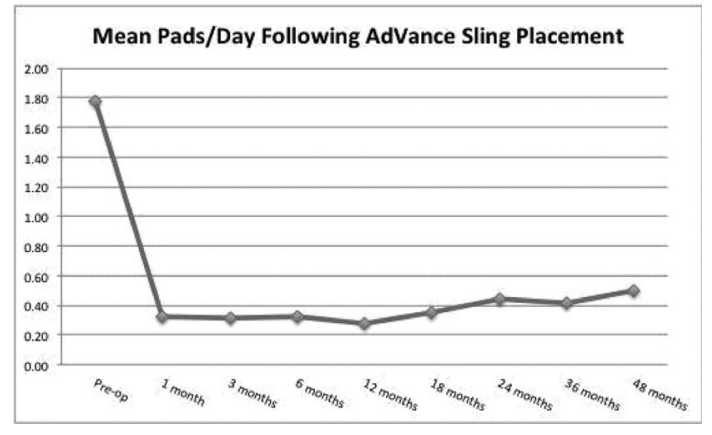

\section{Poster \# NM60}

AUS EROSION IN PATIENTS AFTER RADICAL PROSTATECTOMY WITH AND WITHOUT SALVAGE RADIATION

\section{Amanda Hird, BSc and Sidney Radomski, MD, FRCSC}

University of Toronto, Division of Urology, Toronto Western

Hospital, Toronto, Canada

(Presented by: Sidney Radomski, MD, FRCSC)

Introduction and Objectives: In the last 10 years there has been an increase in the number of patients undergoing radiation after radical prostatectomy (RP). Significant stress incontinence after RP is not uncommon and an artificial urinary sphincter (AUS) is the gold standard of treatment. Our objective was to assess if radiation after RP has increased the rate of erosion and infection in men who have had an AUS in the last 10 years.

Methods: We retrospectively examined 118 patients from December 2001 to January 2012 who underwent a RP with or without postoperative radiation and subsequently had an AUS implanted. We divided the patients into two cohorts, those with AUS implantation from December 2001-December 2006 $(\mathrm{n}=36)$ and those between January 2007-January 2012 (n $=82$ ). We reviewed all patient records for age, cuff size implanted, history of postoperative radiation, previous incontinence surgery, revisions and complications (erosion/infection).

Results: The mean age was similar between groups, 67 years (range: 52-82) in Group 1 and 67 years (range: 50-82) in Group $2(p=0.980)$. The number of patients treated with postoperative radiation was similar between Groups (36\% vs. 32\%, $p=0.640$, respectively). There was no difference in the incidence of erosion between Groups 1 and 2 in those treated with and without radiation ( $p=1.0$ and $p=0.87$, respectively). Similarly, the incidence of infection was similar between Groups 1 and 2 in patients treated with and without radiation $(p=0.305$ and $p=0.359$, respectively). However, the relative risk of erosion was significantly higher overall in those who had radiation compared to those that did not (RR 4.05, 95\% CI 1.115.3).

Conclusions: At our center over the last 10 years, there has not been an increase in the number of patients undergoing AUS after RP and radiation. The incidence of erosion and infection has not increased over the last 10 years. However, as documented in past reports, the relative risk of erosion remains higher in patients who have had radiation.

Funding: No financial funding.

\section{Poster \# NM61}

SURGEON'S IMPRESSION OF URETHRAL STUMP LENGTH DOES NOT CORRELATE WITH URINARY INCONTINENCE AFTER ROBOTIC PROSTATECTOMY

Daniel Hoffman, MD and Ricardo Sánchez-Ortiz, MD

University of Puerto Rico, San Juan, PR

(Presented by: Daniel Hoffman, MD)

Introduction and Objectives: Occasionally during robotic prostatectomy (RP) the urethral stump appears very short and completely retracts into the perineum after division. While it is logical to assume that men with a poor stump would have more incontinence (SUI) and perhaps should receive more postoperative pelvic floor training, the correlation between the surgeon's impression of the urethra and SUI has not been extensively studied. 
Methods: Our cohort consisted of 300 consecutive men who underwent RP by one surgeon and had follow up of $\geq 12$ months (mo). Urethral stump length was subjectively described in each operative note as "good" or "poor." A poor stump was defined as one disappearing into the perineum and barely visualized even despite perineal pressure. The urethral description was correlated with SUI after one year (yr.), time to continence and variables in our prospective database. Continence was defined as zero pads after one yr. Statistical analysis was performed with SPSS.

Results: Of our cohort, 8.7\% (26/300) of men had a urethral stump subjectively described as poor by the surgeon. Patients with a poor stump exhibited a trend for a longer time to continence (6.7 vs. 5.2 mo., $p=0.25$ ) and a higher risk of SUI ( $10.3 \%$ vs. $8.1 \%, p=0.72)$ but these were not statistically significant. Men with a poor stump had a higher mean body mass index (BMI) (29.7 vs. 27.4, $p=0.05)$, higher estimated blood loss (EBL) (242 vs. $242 \mathrm{ml}, \mathrm{p}=0.001$ ) and longer operative times (203 vs. 185 minutes, $p=0.018$ ). In multivariate analysis, only BMI (Odds ratio (OR): 1.13, 95\% confidence intervals (CI): 1.03 to $1.25 \mathrm{p}<0.014)$ and EBL (OR: $1.01,95 \%$ CI 1.005 to 1.015 $\mathrm{p}<0.003)$ correlated with a poor stump. Subjective urethral length did not correlate with age (58.9 vs. 57.6 yr.), prostate weight (43.6 vs. 45.9 grams), bladder neck contracture (0 vs. $1.1 \%$ ), anastomotic leak (4\% vs. $1.8 \%$ ), median follow-up (26.6 vs. $25.1 \mathrm{mo}$.), length of stay, intraoperative or postoperative complications, prior history of voiding dysfunction, diabetes, pathologic stage or history of a positive biopsy at the apex. Conclusions: Men who were characterized as having poor urethral stump at the time of RP were more likely to have a higher BMI, longer operative times and a higher EBL. The surgeon's subjective impression of the urethra was not predictive of the likelihood of developing postoperative SUI. Funding: None.

Clinical Relevance: Male Incontinence

\section{Poster \# NM62}

\section{IMPACT OF OBESITY ON ARTIFICIAL URINARY SPHICNTER FOR THE TREATMENT OF POST-PROSTATECTOMY INCONTINENCE}

Robert Chan, $\mathrm{MD}^{1}$, Chris Graziano, $\mathrm{MD}^{2}$, Jason Scovell, $\mathrm{BS}^{2}$ and Timothy Boone, $\mathrm{MD}, \mathrm{PhD}^{3}$

${ }^{1}$ Department of Urology, Houston Methodist Hospital, Houston, TX; ${ }^{2}$ Baylor College of Medicine, Houston, TX; ${ }^{3}$ Houston Methodist Hospital, Houston, TX

(Presented by: Robert Chan, MD)

Introductions: Obesity can result in worse continence outcomes for certain anti-incontinence procedures such as the male sling. We reviewed the impact of obesity on outcomes of artificial urinary sphincter (AUS) placement for post-prostatectomy incontinence.

Methods: We analyzed the records of 67 male patients during a 17 year period between 1993 to 2009. Patients were stratified by body mass index (BMI). There were 25 patients in the normal group with $\mathrm{BMI}<25$ ), 24 patients in the overweight group with BMI 25 to 30 and 18 patients in the obese cohort with $B M I \geq 30$. Mean age was 69, 66 and 66 years in the normal, overweight and obese groups, respectively. All patients underwent an initial artificial urinary sphincter placement for post-prostatectomy incontinence. Long-term follow-up was obtained through office examination. Medians are reported, and Kruskal-Wallis and chi-square were calculated for significance $(\mathrm{p}<0.05)$.
Results: The mean time from prostatectomy to AUS placement was 49, 40 and 57 months with mean follow-up of 46, 31 and 46 months in the normal, overweight and obese groups. AUS placement resulted in a median decrease of daily pads in normal (4.0), overweight (4.0) and obese men (3.0). Patient BMI did not affect pad use changes $(p=0.54)$. The complication rate was $4 \%$ (one cuff erosion), $12.5 \%$ (three infections) and $5.6 \%$ (one reservoir infection) in the normal, overweight and obese groups, and was not associated with BMI $(p=0.49)$

Conclusions: Obesity did not significantly impact continence parameters after artificial urinary sphincter placement for post-prostatectomy incontinence. In light of available data in literature, AUS should be preferred over male sling in the obese patient population.

Clinical Relevance: Reconstruction

\section{Poster \# NM63}

IMPACT OF MALE URETHRAL SLING EXPLANT VERSUS REVISION IN PATIENTS REOUIRING SECONDARY ARTIFICIAL URINARY SPHINCTER PLACEMENT DUE TO FAILED PRIMARY SLING IN PATIENTS TREATED FOR PROSTATE CANCER

Nilay Gandhi, MD and E. James Wright, MD

The James Buchanan Brady Urological Institute and Department of Urology, The Johns Hopkins School of Medicine, Baltimore, $M D$

(Presented by: Nilay Gandhi, MD)

Introduction and Objectives: Urethral sling placement is commonly used over artificial urinary sphincter for incontinence following prostate cancer treatment, however, many patients require additional therapy. We report our clinical experience assessing urethral sling explant versus sling revision at the time of subsequent artificial sphincter placement following sling failure.

Methods: 22 patients with stress incontinence following prostate cancer treatment underwent AMS 800 artificial urinary sphincter placement after failed urethral sling between November 2006 and July 2013. The AdVance ${ }^{T M}$ sling was used in 14 patients, while eight had an InVance ${ }^{\mathrm{TM}}$ sling. The sling explant and sling revision groups were both composed of 11 patients. Pre- and post-operative evaluation included demographic variables, pre-sphincter pad use, comorbidities, operative time, blood loss, cuff selection, urethral circumference, complication rate and time to initial sphincter revision.

Results: No statistical difference was noted between the explant and revision groups in demographics, pre-sphincter pad use (average 4.9 vs. 4.5), operative time (193 minutes vs. $188)$, estimated blood loss ( 90.9 vs. 75$)$, urethral circumference (6.2 for both), cuff size (4.5) or patients needing revision (6 vs. 4). The type of initial urethral sling used $(p<0.01)$ and proximal cuff placement (100\% explant group vs. $90.9 \%$ revision, $\mathrm{p}=0.04$ ) were noted to be significant. The overall sphincter revision rate was $45.5 \%$ (10 of 22 patients, 55\% explant group vs. $36.4 \%$ revision). Five patients required cuff replacement for atrophy ( 3 in explant group vs. 2 revision) and two patients in the sling explant group required sphincter explant due to infection. With 6.75 years follow-up, the patients undergoing sling explant were found to have a significantly faster time to initial AUS revision (average 123.33 days vs. 996.5, $\mathrm{p}<0.01$ ). The explant group had two patients requiring additional revisions, both having previously received adjuvant radiation therapy. No differences were noted in terms of prostate cancer treatment or prostatectomy technique. 
Conclusions: Patients requiring artificial urinary sphincter after failed urethral sling tend to have a faster time to initial sphincter revision of any cause if the existing urethral sling is explanted rather than revised. Further studies are warranted to assess the impact sling explant has on subsequent sphincter revision rates and long-term continence.

Financial Funding: None.

\section{Poster \# NM64}

\section{TOWARDS A NEW NOMOGRAM AND AN INDEX FOR FEMALE OBSTRUCTION}

Françoise Valentini, MD, PhD and Pierre Nelson, $\mathrm{PhD}$

ER6-Université Pierre et Marie Curie (Paris 06)

(Presented by: Françoise Valentini, MD, PhD)

Introduction and Objectives: Evaluation of obstruction in women remains controversial. Some cutoff values (1) and nomogram (2) have been proposed but without consensus as evaluation of detrusor pressure is difficult. The VBN mathematical micturition model (3) allows evaluating both detrusor contractility (k) and urethral obstruction (gamma) from UDS recordings. Our purpose was to build a nomogram in the plane [pdet.Omax-Omax] allowing an evaluation of the urethral obstruction without any computation.

Methods: Using the VBN model, theoretical computations of intubated flow (catheter $7 F$ ) were performed for various values of initial bladder volume (Vini) [200-600mL], $\mathrm{k}$ [0.25-1.5] and gamma [0-60 cmH2O]. For $n$ values of each parameter $n 3$ (ncube) computations were necessary. Then the curves pdet. Omax (Qmax) were plotted for various values of gamma.

Results: (Fig)

1-The curves pdet.Omax (Omax) were linear of equation pdet. Omax $=\mathrm{A}+\mathrm{B}^{*}$ Omax

2-For the different Vini, it was observed that A and B did not depend of Vini.

3-The straight lines were close to parallel lines with $B=0.52$.

4-The straight lines were regularly spaced out (A was a linear function of gamma).

So, a new index was defined: $A=$ pdet.Omax $-0.52^{*}$ Omax, called WOI (woman obstruction index) with gamaÿ $=(1.16 \mathrm{~A}$ -11.13). Comparison of a posteriori was made between gamma obtained from VBN computation (UDS recordings) and from nomogram (pdet.Omax and Omax). For 125 women without prolapse but with LUTD error was $<5 \%$ : Three examples on Fig (green $=\mathrm{DO}$, black $=$ ISD, blue $=$ normal UDS).

Conclusions: Mathematical modeling allows to build a nomogram for evaluation of urethral obstruction in woman. In addition, an obstruction index for woman (WOI), similar to BOOI in man, is defined. Further studies will be to investigate female bladder outlet obstruction in large populations.

1-Urology 2004;64:675-80; 2-NAU 2000;19:553-64; 3-NAU 2000;19:153-76.

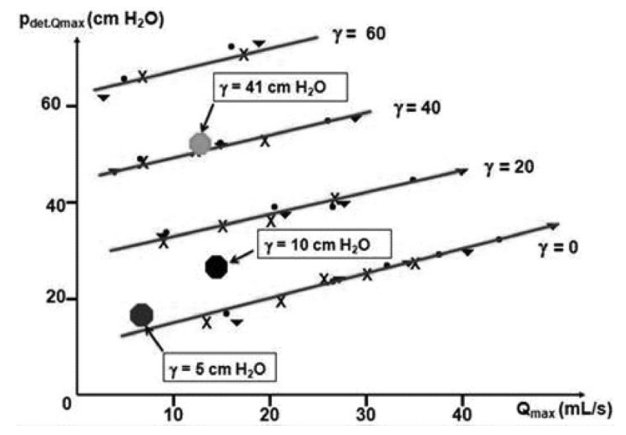

\section{Poster \# NM65}

IS THERE A URODYNAMIC PARAMETER ASSOCIATED WITH DETRUSOR AFTER-CONTRACTION? NEW INSIGHT.

Françoise Valentini, $\mathrm{MD}, \mathrm{PhD}$, Brigitte Marti, $\mathrm{PT}^{1}$, Gilberte Robain, $\mathrm{MD}, \mathrm{PhD}^{2}$ and Pierre Nelson, $\mathrm{PhD}^{2}$

${ }^{1}$ Hôpital Saint Antoine Paris 75012, France; ${ }^{2}$ ER6-Université Pierre et Marie Curie (Paris 06), France

(Presented by: Françoise Valentini, MD, PhD)

Introduction and Objective: Detrusor after-contractions (DAC) are non common in adults (1). Both definition and significance remain discussed. Our purpose was to carry out an analysis of the urodynamic parameters in voidings with DAC and, using the VBN model (2), to make simulations of pathophysiological conditions able to explain both voiding phase and DAC.

Methods: Among a database of patients referred for voiding dysfunction, 60 DAC (5.7\%) were observed during UDS (53 women, seven men). Cystometry was performed with a $7 \mathrm{~F}$ triple lumen catheter allowing the recording of urethral pressure. Criteria for DAC were: post void residual $<30 \mathrm{~mL}$ and increase of detrusor pressure (pdet) $>10 \mathrm{~cm} \mathrm{H2O}$. VBN model was used for analysis of recordings and simulations.

Results: 1-UDS: Onset of DAC (ODAC) occurred during the end of voiding: OODAC $=7.3 \pm 5.7 \mathrm{~mL} / \mathrm{s}$ (while Omax $=18.9 \pm 8.2 \mathrm{~mL} / \mathrm{s}$; $\mathrm{p}<.0001)$. ODAC was associated to an inversion of the slope of pdet curve without any perturbation in flow curve in $47 / 60$. The bladder volume at ODAC was $17.9 \pm 15.4 \mathrm{~mL}$ (i.e. bladder collapse condition). The pressure amplitude of DAC (pDAC) was significantly higher than pdet.Omax: $82.3 \pm 57.4$ vs. $33.1 \pm 16.6 \mathrm{~cm}$ $\mathrm{H} 2 \mathrm{O}$ ( $\mathrm{p}<.0001)$. DO was found in $32 / 60$ (53\%) patients and BOO in $10 / 60$ (17\%). Urgency-frequency was the complaint for $43 / 60$ (72\%) patients. No change of activity of both urethra and rectum was observed during DAC. 2-VBN: Until ODAC voiding was always restored by VBN analysis. After ODAC, the flow curve was restored using the same VBN mechanical parameters (detrusor force and urethral parameter) as in the initial phase of voiding but the increase of pdet could not be restored. The brisk change of pdet slope without any flow perturbation could not be explained by the VBN model or almost spherical bladder model. Neither hypothesis of a great (abnormal) detrusor force or an abdominal straining only effective after voiding nor that of abnormal nervous control before the end of voiding allowed restoring the recorded curves (pressure, flow rate and DAC).

Conclusions: The onset of DAC is the more significant phenomenon. DAC is not associated with BOO but more probably with urgency-frequency syndrome or DO. That latest phenomenon occurring with concomitant bladder wall collapse could lead to a concentration of stresses around the transducer similar to that observed at the tip of a nail. DAC appears as the result of local conditions in an almost empty bladder and thus of weak clinical significance.

1-BJU Int 2002;90:286-93; 2-NAU 2000;19:153-76

\section{Poster \# NM66}

INCREASEING ABDOMINAL PRESSURE DURING BLADDER FILLING IN PEDIATRIC VS ADULT URODYNAMICS

Gillian Wolff, $\mathrm{MD}^{1}$, David Crawley, $\mathrm{MD}^{1}$, Eric Nelson, $\mathrm{MD}^{2}$ and Philip Smith, $\mathbf{M D}^{1}$

${ }^{1}$ Division of Urology, University of Connecticut Health Center, Farmington, CT; ${ }^{2}$ Department of Urology, Connecticut Children's Medical Centerpartment of Surgery, University of Connecticut Health Center, Department of Urology, Connecticut Children's Medical Center

(Presented by: Gillian Wolff, MD) 
Introduction: Central to the treatment of lower urinary tract dysfunction is ensuring a low-pressure reservoir. Impaired compliance with high detrusor pressures is ultimately associated with impaired renal function. Detrusor pressure (Pdet) is the internal bladder pressure attributable to bladder wall tension and, by standard convention, is cystometrically approximated as the measured bladder pressure (Pves) minus an estimation of abdominal pressure (Pabd). Only Pves is directly measured. As an indirect measure of abdominal pressure, Pabd may not accurately reflect the true extrinsic pressure component of Pves. This discrepancy affects the Pdet calculation and can thus yield an erroneous risk assessment. In our practice we have observed a steady rise in Pabd inconsistent with physiologic processes. This could result in falsely reassuring Pdet values. We sought to evaluate the prevalence and severity of this phenomenon in children and adults.

Methods: Sequential urodynamic studies were examined from our pediatric and adult urodynamics units. For each tracing, the Pdet was calculated by standard convention. The change in Pves between the start and end of filling was calculated ( $\Delta$ Pves). This value was subtracted from the standard Pdet for a value labeled "Gap." Gap distributions were determined in pediatric, adult and overall populations. Chi-square analysis was applied to high-risk pressures ( $>=40 \mathrm{~cm} / \mathrm{w})$.

Results: 158 pediatric and 138 adult urodynamic tracings were examined. Median ages were 17 (range 0.3-92) overall; 9.6 (0.321) children and 59 (19-92) adults. Overall median Pdet, $\Delta$ Pves and gap were 6 (2-16), 10 (4-22) and 3 (0-9). In all age groups, Pdet significantly differed from $\Delta$ Pves $(p<0.0001)$, and was significantly greater in children than in adults $(p=0.0008)$. Assuming $\triangle$ Pves is a true indicator of risk, as a detector of "at risk" pressures in children, standard Pdet has a sensitivity and specificity of $88 \%$ and $89 \%$ in children, and positive and negative predictive values of $48 \%$ and $98 \%$.

Conclusions: An artificial depression of Pdet due to steady rise in Pabd was common in our population (18\%). In this pediatric population, Pdet had a less than $50 \%$ predictive value of finding a significant loss of compliance. This effect was rare in our adult population. We conclude that caution should be exercised when determining risk to renal function based on standard Pdet calculations in pediatric urodynamics.

Clinical Relevance: Urodynamics

\section{Poster \# NM67}

\section{URODYNAMIC FINDINGS IN PATIENTS WITH STRESS URINARY INCONTINENCE: A COMPARISON OF DIABETIC VERSUS NONDIABETIC FEMALES}

Rena D Malik, MD, Joshua A Cohn, MD $^{1}$, Sarah L Garvey ${ }^{2}$, Gregory T Bales, $\mathbf{M D}^{1}$ and Doreen E Chung, $\mathbf{M D}^{3}$

${ }^{1}$ University of Chicago Medical Center, Chicago, IL; ${ }^{2}$ Mount Sinai Hospital, Chicago, Il; ${ }^{3}$ University of Chicago Medical Center \& Mount Sinai Hospital, Chicago, IL

(Presented by: Rena D Malik, MD)

Introduction: Urodynamic testing (UDS) is often not performed for isolated stress urinary incontinence (SUI) prior to surgery. Urodynamic findings in females with diabetes mellitus (DM) have not been well-studied. There is controversy as to whether or not classic "diabetic cystopathy" exists and most studies on this topic are not contemporary. The objective was to compare urodynamic findings in diabetic and nondiabetic females presenting with SUI.

Methods: A single center UDS database from 2010 to 2013 was searched for females with complaint of SUI and retrospectively reviewed. Studies were performed according to International Continence Society Standards. For tests of significance, Fisher's exact and Students t tests were performed.

Results: 221 Female patients with SUI underwent UDS from September 2010 to August 2013. Of those, 66 (30\%) patients had DM. Diabetic patients were older (58 years vs. 49 years, $p<0.01)$ and had a higher BMI (34 vs. 31 p < 0.01). Indications for UDS were similar in both groups. Nondiabetics presented with nocturia more often ( $25 \%$ vs. $6 \%, p<0.01)$. Women with DM had significantly higher volume at first sensation, (190 vs. $127 \mathrm{~mL}, p=0.024$ ), increased capacity ( $421 \mathrm{vs.} 507 \mathrm{~mL}, p=0.03$ ), increased compliance (125 vs. $165 \mathrm{~mL} / \mathrm{cmH} 2 \mathrm{O}, \mathrm{p}=0.05)$, decreased detrusor pressure (Pdet) at maximum flow (Omax) (29 vs.36 cmH2O, p=0.04) and increased post void residual (PVR) (26 mL vs. $49 \mathrm{~mL}, \mathrm{p}=0.04)$. (Table 1 ).

Conclusions: In this contemporary series of females with SUI and DM, diabetic females did indeed demonstrate findings characteristic of diabetic cystopathy on UDS, specifically, decreased sensation, increased bladder capacity, decreased Pdet at Omax and elevated PVR. Further studies are required to better examine the clinical significance of these findings and its impact on therapeutic interventions for SUI.

Funding Sources: None

Clinical Relevance: Female Urology-including Incontinence

\begin{tabular}{|c|c|c|c|}
\hline & $\begin{array}{c}\text { NonDiabetics } \\
(n=155)\end{array}$ & $\begin{array}{c}\text { Diabetics } \\
(n=66)\end{array}$ & P Value \\
\hline Mean First Sensation \pm SD $(\mathrm{mL})$ & $127 \pm 129$ & $190 \pm 181$ & 0.024 \\
\hline Mean Capacity \pm SD $(\mathrm{mL})$ & $421 \pm 245$ & $507 \pm 315$ & 0.032 \\
\hline Mean Compliance $\pm \mathrm{SD}(\mathrm{mL} / \mathrm{cmH} 2 \mathrm{O})$ & $125 \pm 109$ & $165 \pm 188$ & 0.054 \\
\hline Mean Qmax (mL/s) & $18 \pm 12$ & $21 \pm 15$ & 0.112 \\
\hline Mean Pdet at Qmax $(\mathrm{cmH} 2 \mathrm{O})$ & $36 \pm 26$ & $29 \pm 19$ & 0.038 \\
\hline Mean Max Pdet $(\mathrm{cmH} 2 \mathrm{O})$ & $56 \pm 35$ & $46.9 \pm 29.3$ & 0.087 \\
\hline Involuntary Contractions ( $\%$ ) & $42(28)$ & $20(31)$ & 0.623 \\
\hline Stress Incontinence $(\%)$ & $92(62)$ & $43(69)$ & 0.346 \\
\hline PVR $\pm S D(m L)$ & $26 \pm 62$ & $49 \pm 103$ & 0.039 \\
\hline
\end{tabular}

\section{Poster \# NM68}

\section{PUDENDAL VS SACRAL NEUROMODULATION FOR PELVIC PAIN}

Kenneth M. Peters, $\mathbf{M D}^{1,2}$, Kim A. Killinger, $\mathbf{M S N}^{1}$, Judith A. Boura, $\mathbf{M S}^{1,2}$, Jason Gilleran, $\mathbf{M D}^{1,2}$ and Jamie Bartley, $\mathrm{DO}^{1}$

${ }^{1}$ Beaumont Health System, Royal Oak, MI; ${ }^{2}$ Oakland University William Beaumont School of Medicine, Rochester, MI

(Presented by: Kenneth M. Peters, MD)

Introduction and Objectives: Chronic pelvic pain (CPP) can improve after sacral neuromodulation, but lead implant at the pudendal nerve may be more effective because of increased afferent stimulation. We evaluated CPP/voiding in patients implanted with a pudendal vs. sacral lead for chronic neuromodulation.

Methods: Adults in our prospective observational neuromodulation study were evaluated. Inclusion criteria were pre-implant CPP $\geq 4$ (0-10; none to severe) on visual analog scale (VAS) and successful staged lead and generator implant. Post-implant, VAS pain scores were reported at three, six, 12 and 24 months. Changes in voiding were measured with 
Validated Interstitial Cystitis Symptom/Problem Indices (ICSIPI). Global Response Assessment (GRA) evaluated overall bladder symptoms. Data were examined with Pearson's Chisquare, Fisher's Exact and Wilcoxon rank tests, and repeated measures analyses.

Results: Of 82 patients (90\% female), 22 (26.8\%) had a pudendal and $60(73.2 \%)$ had a sacral lead placed. Demographics were similar between groups; 14 (64\%) pudendal patients had previously failed sacral stimulation. A higher proportion of pudendal had primary diagnosis of CPP (18.2\% vs. 5.1\%; $\mathrm{p}=0.022$ ) and a lower proportion had urge urinary incontinence (9.1\% vs. 30.5\%; $p=0.024)$. In the pudendal/sacral groups respectively, baseline VAS scores were $7.3 \pm 1.9$ and $6.8 \pm 1.8(p=0.31)$. In those providing three month data, a significantly higher proportion of pudendal patients still reported pain $(19 / 20$ vs. $32 / 48 ; p=0.014)$ and VAS scores were also significantly higher (6.0 vs. $3.3 ; \mathrm{p}=0.004)$. However, VAS scores did not differ between pudendal/sacral groups at any other time point and improved significantly over time in both $(p=0.003$ and $<0.0001$, respectively). ICSI-PI scores did not differ between groups at any time point and both also improved over time ( $p<0.0001$ for both). On the GRA, a lower proportion of pudendal patients reported moderately/markedly improved bladder symptoms at six months (6/15 vs. 31/ $43 ; \mathrm{p}=0.026$ ) but no significant differences were seen between groups at any other measured time point.

Conclusions: The majority of pudendal patients were refractory to sacral stimulation and had pain as their primary diagnosis. Although initially pain improved to a lesser degree in the pudendal group, both groups improved over time suggesting that pudendal neuromodulation may be effective for pelvic pain in the refractory patient.

Funding: MPURE (Philanthropy)

Clinical Relevance: IC and Pelvic Pain-UTI / Inflammatory

\section{Poster \# NM69}

\section{PUDENDAL VS SACRAL NEUROMODULATION FOR URINARY RETENTION}

Kenneth M. Peters, $\mathbf{M D}^{1,2}$, Kim A. Killinger, $\mathbf{M S N}^{1}$, Judith A. Boura, $\mathrm{MS}^{1,2}$, Jason Gilleran, $\mathrm{MD}^{1,2}$ and Jamie Bartley, $\mathrm{DO}^{1}$

${ }^{1}$ Beaumont Health System, Royal Oak, MI; ${ }^{2}$ Oakland University William Beaumont School of Medicine, Rochester, MI (Presented by: Kenneth M. Peters, MD)

Introduction and Objectives: Although sacral neuromodulation improves idiopathic retention, outcomes after stimulating the sacral roots via pudendal afferent pathways are unclear. We compared pudendal vs. sacral neuromodulation in patients with urinary retention.

Methods: Adults in our prospective observational neuromodulation study were evaluated. Inclusion criteria were primary or secondary urinary retention and staged lead and generator implant. Catheter use, voids/day and voided volume were evaluated with diaries between stages and at three, six, 12 and 24 months. Symptoms were also assessed with validated Interstitial Cystitis Symptom/Problem Indices (ICSI-PI) and Global Response Assessments (GRA). Data were examined with Pearson's Chi-square, Fisher's Exact and Wilcoxon rank tests, and repeated measures analyses.

Results: Of 45 patients (80\% female), 15 (33.3\%) had a pudendal and $30(66.7 \%)$ had a sacral lead placed. Demographics were similar between groups; $10 / 15$ (66.7\%) pudendal patients had previously failed sacral stimulation. Almost all in both pudendal and sacral groups reported prior intermittent and/or indwelling catheter use (14/15 and 35/45; $p=0.13)$; the proportions still catheterizing at each follow up were similar between groups. Average voids/day and voided volume did not change significantly over 24 months but groups' scores were comparable at each time point except for between stages where voids/day were lower in pudendal vs. sacral patients $(6.2 \pm 3.6$ vs. $8.7 \pm 3.0 ; p=0.039)$. On the GRA, although the number of patients were small at each follow up, similar proportions reported moderate/marked improvement in bladder symptoms, except at 12 months where $2 / 7(28.6 \%)$ of pudendal and $13 / 16$ (81.3\%) of sacral reported this level of improvement $(p=0.026)$. Composite ICSI-PI scores were also comparable between groups at each time point except for at 12 months where scores were significantly worse in the pudendal group (19.4 \pm 8.0 vs. $9.7 \pm 6.2 ; p=0.015)$. Over time, both pudendal and sacral groups had significant improvements in ICSI-PI composite scores $(p=0.0326$ and $p<0.0001$, respectively) and at each time point the majority in each group indicated satisfaction with treatment.

Conclusions: Even after prior sacral failure, patients with urinary retention may benefit from pudendal lead placement. More study of the impact of pudendal vs. sacral stimulation on urinary retention in larger samples is needed.

Funding: MPURE (Philanthropy)

Clinical Relevance: LUTS / Voiding Dysfunction

\section{Poster \# NM70}

URODYNAMIC SENSORY PARAMETERS DO NOT PREDICT STAGE I INTERSTIM SUCCESS FOR IDIOPATHIC OVERACTIVE BLADDER WITHOUT DETRUSOR OVERACTIVITY

Eugene W Lee, $\mathrm{MD}^{1}$, Justina Tam, BS${ }^{2}$, Chong $\mathrm{H}_{\text {Choe, }} \mathrm{MD}^{1}$, Alvaro Lucioni, $\mathrm{MD}^{1}$, Una Lee, $\mathrm{MD}^{1}$ and Kathleen Kobashi, $\mathbf{M D}^{1}$

${ }^{1}$ Virginia Mason Medical Center, Seattle, WA; ${ }^{2}$ Stony Brook University School of Medicine, Stony Brook, NY

(Presented by: Eugene W Lee, MD)

Introduction: Predictors of success of stage I sacral neuromodulation (SNM) for patients with refractory, idiopathic overactive bladder (OAB) are lacking. We previously showed that if detrusor overactivity (DO) was present, a lower volume at DO predicted SNM failure. However, a large proportion of women presenting with $\mathrm{OAB}$ do not demonstrate detrusor overactivity on urodynamics (UDS), suggesting a sensory etiology. We sought to determine if sensory UDS parameters predicted success of stage I interstim in women with $O A B$ without DO.

Methods: We reviewed all stage I interstim procedures with preoperative UDS for idiopathic OAB without DO from 2000 to 2013. By convention, success was defined as $>50 \%$ subjective improvement in incontinence episodes and/or pad use during the trial period. We compared stage I successes and failures in terms of demographic and clinical characteristics, as well as UDS sensory parameters including first sensation of filling (FSF), capacity and first sensation ratio (ratio of FSF to capacity) to determine if we could find any predictors of stage I success.

Results: Of 51 total patients identified who underwent stage I SNM with no evidence of DO on preoperative UDS, 38 were successful (75\%). Age, parity, diabetes, body mass 
index, smoking status and postmenopausal state were not significantly different between successes and failures. Mean FSF was slightly lower for failures $(77 \mathrm{~mL})$ versus successes $(88 \mathrm{~mL})$, but this was not significant. The average maximum capacity was also similar at $351 \mathrm{~mL}$ for successes versus $364 \mathrm{~mL}$ in failures. Mean first sensation ratio was also not significantly different ( 0.23 for successes versus 0.21 for failures).

Conclusions: UDS remain an important part of the diagnostic evaluation of the patient with refractory $O A B$. However, in our series of patients with idiopathic OAB without DO, UDS sensory parameters were not predictive of stage I SNM success.

Clinical Relevance: Urodynamics

\section{Poster \# NM71}

\section{NATIONAL TRENDS IN THE USAGE OF SACRAL NERVE} STIMULATION AMONG MEDICARE BENEFICIARIES

Melissa Laudano, $\mathbf{M D}^{1}$, Stephan Seklehner, $\mathbf{M D}^{2}$, Alexis Te, $\mathrm{MD}^{1}$, Steven Kaplan, $\mathrm{MD}^{1}$, Bilal Chughtai, $\mathrm{MD}^{1}$ and Richard Lee, $\mathbf{M D}^{1}$

${ }^{1}$ Department of Urology, Weill Medical College of Cornell University, New York, NY; ${ }^{2}$ Department of Urology, Landesklinikum Baden-Mödling, Baden, Austria

(Presented by: Melissa Laudano, MD)

Introduction and Objectives: Sacral nerve stimulation (SNS) (InterStim ${ }^{\mathbb{R}}$, Medtronic, Inc., Minneapolis, MN) has been used as a second-line therapy for both urinary and bowel control. There is limited data in the literature regarding trends of utilization of SNS to explore precisely who is treated with SNS. We sought to explore disparities in the utilization of SNS among Medicare beneficiaries.

Methods: A 5\% national random sample of all Medicare claims for 2001, 2004, 2007 and 2010 was queried to identify patients of interest. All patients with an International Classification of Diseases, 9th Edition (ICD-9) diagnosis code that was a potential indication for SNS system were included. Patients who underwent SNS implantation were identified with Current Procedure Terminology (CPT-4) codes. Statistical tests used included the $\chi^{2}$ and Fisher tests, as well as multivariate logistic regression analyses using SAS v9.3 (SAS Institute Inc., Cary, NC) and SPSS v20 (IBM Corp., Armonk, NY).

Results: A total of 3,198,300 patients were identified with a diagnosis that could potentially be treated with SNS. Of these patients, 14,780 patients underwent treatment with SNS $(0.46 \%)$. The percentage of patients diagnosed who ultimately underwent treatment increased from $0.02 \%$ to $0.75 \%$ ( $p<0.001$ ) over the 10-year study period. On logistic regression analysis, women $(O R=4.60, \mathrm{p}<0.0001)$ and patients aged less than 65 years $(\mathrm{OR}=1.00$, compared to older age groups $\mathrm{OR}=0.29-0.44$, $\mathrm{p}<0.0001$ ) were more likely to be treated with SNS. Non-white individuals $(O R=0.48, p<0.0001)$ and those living in the western U.S. (OR $=0.55, p<0.0001)$ were less likely to receive surgical treatment (see Table).

Conclusions: The usage of SNS among patients diagnosed with a potential indication has significantly increased among Medicare beneficiaries over a 10-year period. Patients were more likely to be treated with SNS if they were female, white, were $<65$ years of age and lived outside of the west coast of the US. Knowledge of these data might promote more equal access to SNS nationwide.

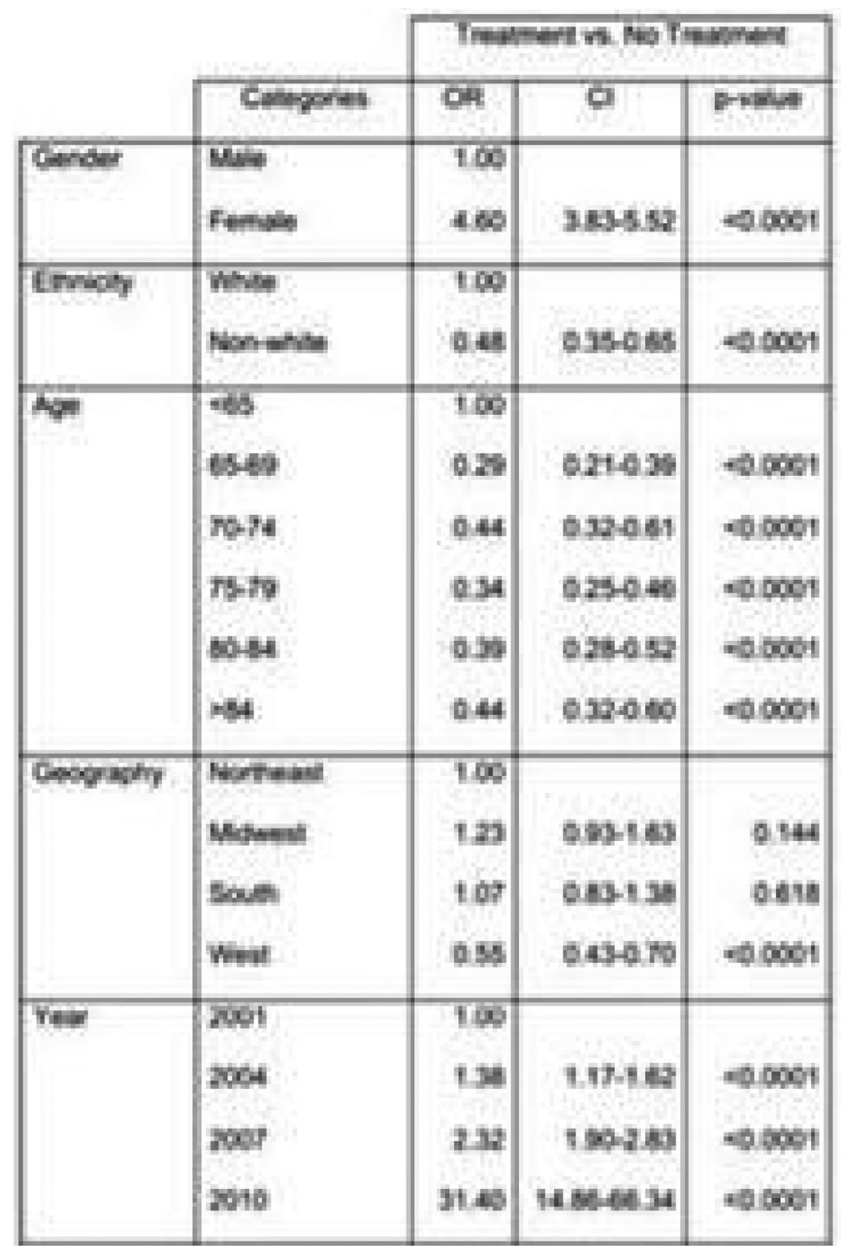

\section{Poster \# NM72}

PATIENTS UNDERGOING SACRAL NEUROMODUOLATION FOR URINARY SYMPTOMS UNDER-REPORT CONCOMITANT BOWEL SYMPTOMS TO THEIR UROLOGIST

Joceline Liu, $\mathbf{M D}^{1}$ and Stephanie Kielb, $\mathbf{M D}^{2}$

${ }^{1}$ Northwestern University, Feinberg School of Medicine;

${ }^{2}$ Northwestern University, Feinberg School of Medicine, Chicago, IL

(Presented by: Joceline Liu, MD)

Introduction and Objectives: Overactive bladder (OAB) symptoms are common, with a prevalence of $12 \%$ in men and women, with incidence increasing with age. Sacral nerve stimulation (SNS) is an FDA-approved device for treating both refractory urinary symptoms and fecal incontinence. In patients presenting to urology clinic for evaluation of refractory urinary symptoms, the history of concomitant bowel symptoms may make SNS a more attractive and comprehensive treatment plan. This study aims to examine the prevalence of concurrent bowel symptoms in patients undergoing SNS with a chief presenting complaint of urinary symptoms.

Methods: Ten patients undergoing SNS implantation with urinary complaints on presentation to urology clinic were prospectively enrolled and surveyed. Patients were questioned by the physician regarding urinary and bowel symptoms and pelvic pain. The patient also completed surveys including Incontinence Impact Questionnaire (IIO-7), Interstitial Cystitis 
Symptoms and Problem Indices (ICSI/ICPI), Fecal Incontinence Severity Index (FISI) and Wexner Constipation Questionnaire (WCO). Patient-reported symptoms were correlated with validated questionnaire results.

Results: Of 10 patients, two patients endorsed significant bowel symptoms with physician questioning in pre-operative clinical evaluation (20\%). With questionnaires (FISI, WCO), five patients $(50 \%)$ reported moderate bowel symptoms (mean FISI 12 , WCQ 11.75), and two additional patients (20\%) reported mild bowel symptoms (mean FISI 7, WCO 7). Of the two patients endorsing bowel symptoms to the physician, one scored moderate symptoms and the other, mild. $80 \%$ patients scoring moderate bowel symptoms on survey denied any bowel symptoms to the physician.

Conclusions: Our data suggests that patients with refractory urinary symptoms under consideration for SNS implantation may have underlying concurrent bowel symptoms that are not captured on preoperative evaluation despite detailed physician questioning. There may be a role for use of validated questionnaires to better characterize preoperative bowel and bladder symptoms prior to SNS and follow postoperative symptom control and improvement.

Supported by the Evergreen Invitational Women's Health Grants

Clinical Relevance: LUTS / Voiding Dysfunction

\section{Poster \# NM73}

\section{EFFICACY OF SACRAL NEUROMODULATION IN PATIENTS OVER THE AGE OF 70}

Chad Hubsher, MD, Robert Jansen, MD and Stanley Zaslau, MD West Virginia University, Morgantown, WV

(Presented by: Chad Hubsher, MD)

Introduction: Lower urinary tract dysfunction refractory to pharmacologic therapy frequently occurs in the elderly. However, reports that address the success of surgical intervention in this patient population are lacking. In an effort to determine the effectiveness of sacral neuromodulation in the elderly population, we report our long-term results, patient satisfaction and reoperation rates in this patient population.

Methods: We retrospectively reviewed patients at our institution that underwent InterStim ${ }^{\circledR}$ sacral neuromodulation implantation for lower urinary tract dysfunction at the age of 70 , or greater. We assessed the long-term outcome, device effectiveness and incidence and cause of surgical reintervention.

Results: Sacral neuromodulation devices were implanted in 23 patients who were over the age of 70 (mean age 76, range 70 to 82 years old) at date of implantation, and continue to follow up with us regularly. Indications for implantation were urinary urgency, frequency and/or urge incontinence in $74 \%$ of patients, and idiopathic urinary retention in $26 \% .13$ patients (57\%) continue to benefit from InterStim ${ }^{\circledR}$ sacral neuromodulation, with a mean follow up from implantation of 55 months. Four of these patients underwent revisions for battery failure and two were revised for lead migration following a fall. 10 patients (43\%) did not achieve the desired benefit of the implanted InterStim ${ }^{\circledR}$ device, which was subsequently explanted in six patients that remained surgical candidates.

Conclusion: Sacral neuromodulation implantation is a minimally invasive procedure with a perceived benefit in an elderly population. Patients should be counseled on the revision and explantation rates prior to undergoing surgery.

Clinical Relevance: Geriatric Urology

\section{Poster \# NM74}

PHOTOVAPORIZATION OF THE PROSTATE WITH THE 180W XPS GREENLIGHT ${ }^{\text {TM }}$ LASER FOR PATIENTS IN URINARY RETENTION

Daniel Lee, $M D$, Malte Rieken, $\mathrm{MD}^{1}$, Fujun Zhao, $\mathrm{MD}^{1}$, Heike Pueschel, $\mathrm{RN}^{2}$, Bilal Chughtai, $\mathrm{MD}^{1}$, Steven Kaplan, $\mathbf{M D}^{1}$, Alexis Te, $\mathrm{MD}^{1}$, Alexander Bachmann, $\mathbf{M D}^{2}$ and Richard Lee, $M D^{1}$

${ }^{1}$ Cornell, NY, NY; ${ }^{2}$ University Hospital Basel

(Presented by: Daniel Lee, MD)

Introduction and Objectives: Photovaporization of the prostate (PVP) with the Greenlight ${ }^{\mathrm{TM}}$ laser is an established treatment option for the treatment of male LUTS due to benign prostate enlargement. Despite the widespread use of the technique, data on outcomes in patients with preoperative urinary retention are scarce. We aim to compare intermediateterm results of patients with vs. without preoperative retention after 180W XPS-PVP.

Methods: A series of 401 patients who underwent PVP with the 180W XPS Greenlight ${ }^{\mathrm{TM}}$ laser (American Medical Systems, Inc., Minnetonka, MN) from July 2010 to June 2013 at two institutions was retrospectively studied. Perioperative parameters as well as postoperative functional results and complications were compared for those with vs. without preoperative urinary retention.

Results: Of 401 patients, 140 patients (34.9\%) were in retention at time of surgery. Patients in retention at time of surgery were older (75.5 vs. 71.1 years; $p=0.04$ ) and had a larger prostate volume ( 86.0 vs. $52.5 \mathrm{ml} ; \mathrm{p}<0.001)$ than patients without retention. Median operating time (70.0 vs. $60.0 \mathrm{~min} ; \mathrm{p}=0.002)$, lasing time ( 44.0 vs. $34.0 \mathrm{~min} ; \mathrm{p}=0.001)$, applied energy ( 376 vs. $261 \mathrm{~kJ} ; \mathrm{p}=0.001)$ and number of fibers used (1.5 vs. 1.0; $\mathrm{p}<0.001)$ was significantly higher in patients in retention. During a follow-up of two years, patients with preoperative retention had a significantly higher rate of postoperative retention $(10.0 \%$ vs. $3.4 \% ; p=0.01)$ and reoperation (3.6\% vs. $0.4 \% ; \mathrm{p}=0.02$ ) although the risk of postoperative urinary tract infection and urethral stricture was similar to patients without preoperative retention.

Conclusions: PVP with the 180W XPS Greenlight ${ }^{\mathrm{TM}}$ laser appears as a safe and effective treatment for patients with preoperative retention. Such patients required longer operative times and more re-intervention, but the risks of other complications were not increased.

Clinical Relevance: LUTS / Voiding Dysfunction

\begin{tabular}{|c|c|c|c|}
\hline Variable & No priar nelention & Redention & P-value \\
\hline $\mathrm{N}(\%)$ & $261(65.1 \%)$ & $140(34.9 \%)$ & \\
\hline Medisn abe, youn & 71.1 & 75.5 & 0.04 \\
\hline $\begin{array}{l}\text { Madian proctuate } \\
\text { volumis, con? }\end{array}$ & 525 & 86 & 00.01 \\
\hline $\begin{array}{l}\text { Madian IPSS scom, } \\
\text { (IOR) }\end{array}$ & $20(14-24)$ & $21(18-25)$ & $\infty 0.01$ \\
\hline $\begin{array}{l}\text { Median prooperative } \\
\text { Qmax, mils (IOR) }\end{array}$ & $8.6(5.5-11)$ & $6(4.7-9.7)$ & 0.02 \\
\hline $\begin{array}{l}\text { Median openting } \\
\text { room time, min }\end{array}$ & 60 & 70 & $<0.01$ \\
\hline Median laving time & 34 & 41 & $<0.01$ \\
\hline Energy used, kJ & 261 & 376 & $\infty .01$ \\
\hline Nunber of fibers usod & 1.0 & 1.5 & $\propto 0.01$ \\
\hline $\begin{array}{l}\text { Posteperative } \\
\text { retention, } \mathrm{N}(\%)\end{array}$ & $9(3.4 \%)$ & $14(10006)$ & 0.01 \\
\hline Reoperation, N(\%) & $1(0.4 \%)$ & $5(3.6 \%)$ & 0.02 \\
\hline $\begin{array}{l}\text { Median postoperative } \\
\text { Qmax, mL/s (IOR) }\end{array}$ & $12.1(7.1-19)$ & $8.9(5.2-14.7)$ & $<0.01$ \\
\hline $\begin{array}{l}\text { Modian pootloperative } \\
\text { PVR, mL. (OQR) }\end{array}$ & $26(0-81)$ & $65(20-158)$ & $<0.01$ \\
\hline
\end{tabular}




\section{Poster \# NM75}

\section{SAFETY AND EARLY OUTCOMES FOLLOWING THULIUM} VAPOENUCLEATION OF THE PROSTATE IN NORTH AMERICA

Shane Pearce, $M D^{1}$, Joseph Pariser, $M^{1}$, Olufenwa Famakinwa, $\mathbf{M D}^{1}$ and Doreen Chung, $\mathbf{M D}^{2}$

${ }^{1}$ Section of Urology, University of Chicago, Chicago, IL; ${ }^{2}$ Division of Urology, Mount Sinai Hospital, Chicago, IL

(Presented by: Shane Pearce, MD)

Introduction: Thulium VapoEnucleation (ThuVEP) has been studied in Asia and Europe and has been shown to be safe and effective even for larger prostates. The aim of this study was to describe initial experience with this technique in a North American (NA) patient population.

Methods: From December 2010 to September 2013, 18 men underwent ThuVEP using the CyberTM ${ }^{\mathbb{R}}$ laser. We performed a retrospective chart review of patient demographics, comorbidities, intraoperative parameters, complications, lab values and post-operative outcomes including maximum flow rate (Omax), post-void residual(PVR), International Prostate Symptom Score(IPSS) and quality of life(OoL) score. Patients were evaluated at one, three, six and 12 months. Statistical analysis was done using Wilcoxon signed ranks test.

Results: Mean age was $71 \pm 9$ years and prostate size $127 \pm 68$ cc's. Baseline mean IPSS was $20 \pm 10$, OoL $5 \pm 2$, PVR $428 \pm 282 \mathrm{~mL}, \mathrm{PSA} 6.8 \pm 5.2$ and $\mathrm{Omax} 1.6 \pm 2.3 \mathrm{~mL} / \mathrm{s}$. Indications for surgery included retention in 15 (83\%) patients. Median Charlson Comorbidity Index was 5(IOR 4-5.75) and 10 (56\%) patients were on anticoagulation. Mean laser time and energy used were $54 \pm 24 \mathrm{~min}$ and $285 \pm 149 \mathrm{~kJ}$. Mean operative time was $180 \pm 74 \mathrm{~min}$. Mean change in hemoglobin ( $\mathrm{Hb})$ was $-0.7 \pm 0.9 \mathrm{~g} / \mathrm{dL}$. No significant change in mean serum sodium was seen $(-0.7 \pm 0.4 \mathrm{mmol} / \mathrm{L}, \mathrm{P}=0.58)$. Most patients went home the day of surgery with mean hospital stay $1 \pm 1$ day. Mean catheter time was $5 \pm 3$ days. Complications occurred in 2 (11\%) patients. One patient developed clot retention requiring catheterization and bedside irrigation. One patient with cardiac comorbidity had blood transfusion for anemia although preoperative and postoperative Hb's were 8.4 and $8.8 \mathrm{~g} / \mathrm{dL}$. Improvements were seen in Omax, PVR, IPSS and OoL score at each time interval (Fig 1). Of 15 patients that initially presented in urinary retention, all with follow-up were voiding at last visit. Conclusions: In this initial NA study, ThuVEP appears to be a safe and effective treatment for BPH, even in patients with large prostates and high comorbidity. There were few complications, minimal hospitalization and outcomes were durable to 12 months. Longer follow-up and larger numbers are necessary to confirm these findings.

Funding Source: None

Clinical Relevance: LUTS / Voiding Dysfunction

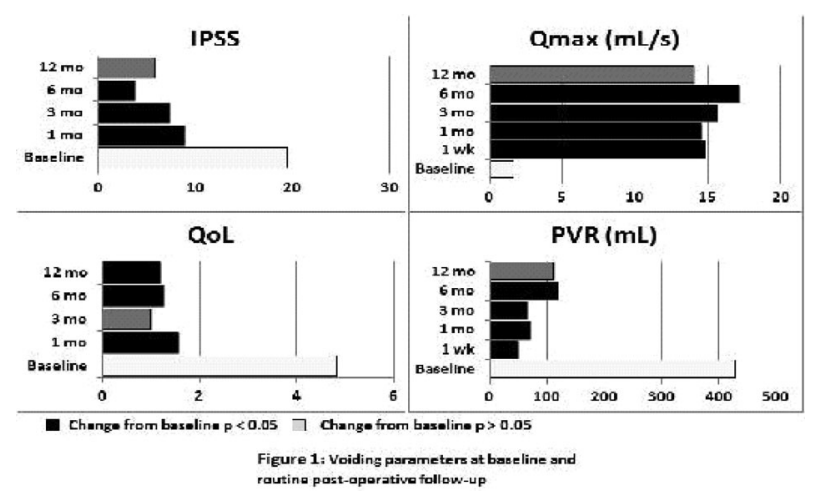

\section{Poster \# NM76}

\section{THE URINARY CATHETER-TO-TRANSURETHRAL RESECTION TIME IN MEN WITH URINARY RETENTION}

Katherine Brewer, MD, Steven Weissbart, MD, Wayland $\mathrm{Wu}, \mathrm{MD}$ and Neil Grafstein, MD

Icahn School of Medicine at Mount Sinai

(Presented by: Katherine Brewer, MD)

Introduction: Catheter associated urinary tract infections (CAUTI) are associated with significant patient morbidity and associated health care costs. As such, the Joint Commission on the Accreditation of HealthCare Organizations (JCAHO) has implemented a strategic plan to reduce CAUTI's by advising indwelling bladder catheters to be removed as soon as possible. In the inner-city community, one group of patients who temporarily require bladder catheterization are men with urinary retention/bladder outlet obstruction (BOO) who are pending Transurethral Resection of the Prostate (TURP). Within this patient population, one group of patients who have an elevated risk of prolonged catheterization are under-insured men with urinary retention. As such, we investigate the urinary catheter-to-Transurethral resection time in underinsured men with urinary retention/BOO, that is, the time interval a man spends with an indwelling urinary catheter pending a TURP.

Methods: The records of 374 men who underwent TURP from 1/ 2007-2/2013 at our urban hospital were reviewed. A member from our billing department was selected and asked to score insurance quality into two groups: insured and underinsured. The medical records of underinsured patients were investigated to determine the duration of time a patient spends with an indwelling for catheter. Only patients with a specific date of foley catheter insertion secondary to urinary retention were included in the cohort.

Results: 144 of 374 men (38\%) required bladder catheterization prior to TURP. 17/144 (12\%) were scored as being underinsured. The mean patient age at the time of TURP in these men was 70 (range 53-89). The mean catheter-to-Transurethral resection time in this group was 150 days (SD 195).

Conclusions: As prolonged bladder catheterization is associated with morbidity and unnecessary healthcare costs, further research is required to investigate ways of decreasing the urinary catheter to transurethral resection time. With the implementation of the affordable healthcare act, managed Medicaid enrollment is expected to increase, ultimately expanding the underinsured patient population. In the ever changing healthcare system, with its associated time and financial constraints, minimizing the catheter duration would be imperative and catheter-to-Transurethral resection time may be considered a metric of quality measure for patient care. Clinical Relevance: BPH

\section{Poster \# NM78 \\ OUTCOMES FOR 532NM LASER PROSTATECTOMY IN IMMUNOCOMPROMISED PATIENTS FOR BPH}

Ashley Winter, MD, Charles Osterberg, MD, Nicholas Hauser, MD, Daniel Lee, MD and Benjamin Choi, MD Cornell, NY NY

(Presented by: Ashley Winter, MD)

Introduction and Objective: We aimed to characterize the outcomes in photovaporization of the prostate (PVP) for those who are immunocompromised compared to those who are not. 
Methods: A series of 213 consecutive patients underwent PVP using the GreenLight ${ }^{\mathrm{TM}}$ laser system (American Medical Systems, Inc., Minnetonka, MN) from 2005 to 2012 and had data regarding immunocompetency. Immunocompromised (IC) status was considered if patients were on any immunosuppression therapy for organ transplants or had a diagnosis of HIV or diabetes. Data was collected on patient demographics, International Prostate Symptom Score (IPSS), maximum flow rate (Omax), postvoid residual (PVR) and prostate volume were recorded.

Results: Of the 213 patients, 90 men (42.2\%) were IC, with eight patients on immunosuppressive therapy for liver or kidney transplants, six patients on antiretroviral medications for HIV and 77 patients with diabetes. Within a median follow-up of 13.3 months (IOR 5-28.3), there were no significant differences in demographic baseline characteristics, except that IC had higher median IPSS scores (16 vs. $20, p=0.047$ ). The median laser time and energy used were not significantly different between the two groups. There were no significant differences in the rate of postoperative UTI (5.7\% vs. 3.3, p=0.7), however one IC patient was hospitalized for urosepsis. There were no differences in postoperative retention or reoperation rates. Both groups showed improvements in voiding parameters (Omax and PVR), but there were no differences in the relative amount of improvement. There was a significant improvement in voiding symptoms in the IC group compared to the nonimmunocompromised, with median improvement of 10 points on the IPSS scale (compared to $6, \mathrm{p}<0.01$ ).

Conclusions: In our series, PVP was safe and effective. Durable improvements in symptoms and objective parameters were achieved in immunocompromised patients with the laser systems, with similar rates of postoperative UTI.

Clinical Relevance: LUTS / Voiding Dysfunction

\begin{tabular}{|c|c|c|c|}
\hline \multicolumn{4}{|c|}{ 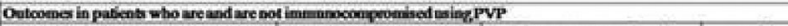 } \\
\hline Vuriable & 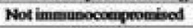 & Imimanoconpromibed & prelue \\
\hline N(6) & $123(57.5 \%)$ & $90(12.2 \%)$ & \\
\hline Molian agen youn (QgR) & $70(63-70)$ & $n(62.56 .9)$ & 0.83 \\
\hline Motian BMa, (OQRe) & $26(24.4-28.6)$ & $26.7(24.2-313)$ & 0.08 \\
\hline Proopendive urinany rotention, N( $)$ & $19(17.1 \%)$ & $7(11.796)$ & 0.19 \\
\hline 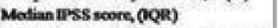 & $16(10-25)$ & $20(14-26)$ & 0.047 \\
\hline Motline proopendive Qmax, al $/ s(O Q R)$ & $75(5-10)$ & $7.2(5-11)$ & 0.98 \\
\hline Motian proopernative PVR, mL.(OQR) & $137(71-236)$ & $138.5(52.5-200)$ & 0.70 \\
\hline 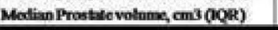 & $50.6(52.7-80)$ & $50(30.2-70)$ & o.ss \\
\hline \multicolumn{4}{|l|}{ Operative } \\
\hline 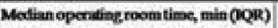 & $60(40-85)$ & $60(38-73)$ & 0.75 \\
\hline Mothan lasing time, mino (OQR) & $33(20-46)$ & $34,4(2 t-50)$ & 0.68 \\
\hline Pacrog wed, kJ (QQ⿱中⿰㇀丶㇀ & $177(98-271)$ & $156(104-238)$ & 0.23 \\
\hline Number offinen uned & 1.0 & 1.0 & 0.17 \\
\hline \multicolumn{4}{|l|}{ Postoprasve } \\
\hline Postopentive retention, $\mathrm{N}(\%)$ & 0 & $2(2.25)$ & \\
\hline Reoperation, N(W) & $2(1.00)$ & 0 & 0.78 \\
\hline Postopentive Un, N(G) & $7(5.796)$ & $3(3.3 \%)$ & 0.74 \\
\hline Motian poskependive IPSS, OQRe) & $9(6-12)$ & $s(3-9)$ & $\infty .01$ \\
\hline Modian poskepentive $\mathrm{Qmax}, \mathrm{mL} / \mathrm{s}$ & $13.5(9-18.2)$ & $12.3(8-15)$ & 0.67 \\
\hline Mothan postopenative PVR, miL(COQR) & $5(0-26)$ & & 0.19 \\
\hline \multirow{2}{*}{\multicolumn{4}{|c|}{ 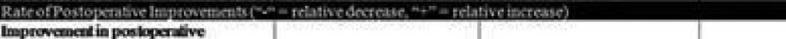 }} \\
\hline Improverned in pos icperitive & & & \\
\hline 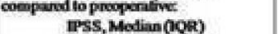 & & & \\
\hline Qmax, Motian (00\%) & $\cdot 8(1-\cdot 17)$ & $25(0-6)$ & 0.11 \\
\hline PVR, Medianegé) & $128(213-60)$ & $-118(288-39)$ & 0.51 \\
\hline
\end{tabular}

\section{Poster \# NM79}

EXPLORING POTENTIAL PREDICTORS OF HERNIA AFTER LAPAROSCOPIC PROCEDURES SUCH AS ROBOTIC ASSISTED RADICAL PROSTATECTOMY (RARP)

Jason Hafron, $\mathrm{MD}^{1,2}$, Scott Pew, BS ${ }^{2}$, Behdod Poushanchi, BS ${ }^{2}$, Jay Hollander, $\mathrm{MD}^{1,2}$, Kim A. Killinger, $\mathrm{MSN}^{1}$,

Mary Coffey, $\mathrm{PhD}^{1}$ and Kenneth M. Peters, $\mathbf{M D}^{1,2}$

${ }^{1}$ Beaumont Health System, Royal Oak, MI; ${ }^{2}$ Oakland University William Beaumont School of Medicine, Rochester, MI

(Presented by: Scott Pew, BS)
Introduction and Objectives: The long term incidence of hernia as a consequence of laparoscopic procedures is not well known. We explored incidence and predictors of hernia after robotic assisted radical prostatectomy (RARP).

Methods: Adult men that had RARP for prostate cancer between 2003 and 2012 were reviewed for medical/surgical data. Hernia type, location (body diagram) and repair were assessed via mailed survey. Hernia/no hernia groups were compared using Wilcoxon Rank Sum Test, Chi-square test and pooled t tests.

Results: Of 577 men (mean age $61.5 \pm 6.6$ years; median body mass index-BMI 27.2), 450/570 (79\%) had stage T2 cancer. Few had any intraoperative $(13 / 577 ; 2.3 \%)$ or postoperative complications (63/577; 11\%). At survey completion, time since RARP ranged from 0.6 to 10.6 years. Of 577, 93 (16.1\%) reported one $(n=82)$, two $(n=9)$ or three $(n=2)$ postoperative hernias at (range) three days to 9.06 years after RARP. In men that developed a hernia, 23 had a history of herniorrhaphy prior to RARP. 48/577 men (8\%) had developed at least one hernia at an incisional site (34 men indicated the umbilical region) that was diagnosed at (median) 1.2 years after RARP. 42/577 (7\%) developed at least one inguinal hernia. 57/93 men had surgical repair of 68 hernias; 23 umbilical, 36 inguinal, six other port and three "other location" hernias. Hernia/no hernia groups were compared on preoperative diabetes, smoking, prior abdominal surgery and herniorrhaphy, length of time since RARP, pathological stage, age, operative time, blood loss, drain type, intraoperative and postoperative complication and BMI, but no statistically significant differences were found. There was a tendency towards increasing hernia rates with worse ASA physical status classification score but this did not reach statistical significance $(p=.062)$. However, men that developed a hernia had a larger median prostate weight than those that did not develop a hernia (43.5 vs. 38 grams; $p<.001$ ). When men were grouped by incisional hernia vs. no hernia, prostate weight was again significantly different (median 45 gm vs. 38 gm; $\mathrm{p}=.001$ ).

Conclusions: Although hernia is a known consequence of laparoscopic procedures including RARP and sacral colpopexy, this analysis of over 550 surgeries failed to demonstrate any association with predicted risk factors such as diabetes, smoking and BMI. Further study into the cause of hernia after robotic procedures needs to be undertaken.

Funding: MPURE (Philanthropy)

Clinical Relevance: Geriatric Urology

\section{Poster \# NM80}

EFFICACY OF PERCUTANEOUS TIBIAL NERVE STIMULATION IN OCTOGENARIANS FOR OVERACTIVE BLADDER SYMPTOMS

Chris Tenggardjaja, MD, Adrienne Quirouet, MD and Howard Goldman, MD

Cleveland Clinic, Cleveland, Ohio

(Presented by: Chris Tenggardjaja, MD)

Introduction and Objectives: The prevalence of overactive bladder symptoms (OAB) has been described as high as $42 \%$ in some studies for patients over 75 years of age. Percutaneous tibial nerve stimulation (PTNS) has been utilized for treatment of OAB. No studies have evaluated its efficacy in the elderly. Our main objective is to evaluate the efficacy of PTNS in octogenarians.

Methods: At Cleveland Clinic from 2010 through 2012, patient data was collected in a prospective manner in a single staff member's clinic (HBG). After IRB approval, patients were 
identified for retrospective analysis by CPT codes 64566/64999. Exclusion criteria included patients who did not receive PTNS treatment or did not have OAB symptoms. Patient demographics included BMI, cystoscopic and urodynamic evaluation, prior incontinence surgeries and treatment. Daytime frequency was standardized as patient reported voids over a 16 hour period. Patient success was defined by improvement after a standard 12 week course of PTNS, continuation of maintenance therapy or subjective improvement on IIO-7/UDI-6 questionnaire scoring. Statistical analysis was performed using Fisher's exact and Mann Whitney tests.

Results: A total of 50 charts were reviewed for this study. 31 patients met inclusion criteria. 12 patients were 80 years or older (mean 84 years \pm 4.5 ) at PTNS treatment. The octogenarian group's symptoms included a median daytime frequency of 10 voids (range 5-16) and median nocturia of four voids (range 1-6). 8/12 patients underwent urodynamic evaluation and of these, $3 / 8$ patients (37.5\%) had detrusor overactivity. Patient comorbid status was characterized using BMI (median $27.8 \mathrm{~kg} /$ m $2 \pm 5.7)$ and median age-adjusted Charlson Comorbidity Index (CCI) (median $7 \pm 1.1$ ). $50 \%$ of octogenarians demonstrated improvement over a 12 week period of PTNS. BMI was the only significant difference between responders and non responders ( 24.2 vs. $32.8 \mathrm{~kg} / \mathrm{m} 2 \mathrm{p}=0.015)$. The significant difference between the octogenarian and an under 80 years of age cohort was CCI (median 7 vs $3 p<0.05$ ). Response to treatment was not statistically different among octogenarians vs. the younger cohort ( $50 \%$ vs $63 \% \mathrm{p}=0.7$ )

Conclusions: To our knowledge this is the first study to evaluate the efficacy of PTNS in octogenarians. PTNS can be used effectively in elderly patients with refractory $O A B$ symptoms. Further studies will be needed to evaluate prognostic indicators for effectiveness of PTNS in this population. Clinical Relevance: Geriatric Urology

\section{Poster \# NM81}

\section{THE USE OF ONABOTULINUMTOXIN A IN PATIENTS OVER THE} AGE OF 70

Anne Sammarco, MD, MPH, Elizabeth Ferry, MD, Dhruti Patel, MD, Adonis Hijaz, MD and Sangeeta Mahajan, MD

University Hospitals Case Medical Center

(Presented by: Anne Sammarco, MD, MPH)

Introduction and Objectives: Despite the accepted efficacy of intradetrusor injection of OnabotulinumtoxinA for detrusor overactivity (DO), little research exists on the effect of patient age with regards to treatment outcomes. Most research studies cut-off patient recruitment at 70 to 75 years old, and as a result, almost all of the studies demonstrating the excellent outcomes and low associated side effects of this treatment are in patients below this age range. To date, the outcomes and side-effect profile of intra-detrusor botulinum toxin injection for the treatment of DO in patients over the age of 70 years has not been examined. To evaluate the outcomes of patients over the age of 70 who have received intra-detrusor botulinum toxin injection for the treatment of DO as compared with patients under the age of 70 .

Methods: A retrospective chart review of all patients in our practice 18 years of age or older who received OnabotulinumtoxinA detrusor injections between January 1, 2008 and January 31, 2013, for the treatment of DO was conducted. Fifty charts were reviewed, 17 from patients over the age of 70 , and 33 charts representing patients between the ages of 18 and 69.
The Fisher's Exact Test was utilized to evaluate the data. Outcomes evaluated include subjective improvement of symptoms, post injection urinary retention and urinary tract infection within 30 days post-treatment.

Results: In the over 70 age group, $70.6 \%$ of patients experienced symptom improvement, as compared with $87.9 \%$ in the under 70 age group $(p=0.2420)$. Infection was recorded in $17.6 \%$ of those over 70 years old and in $21 \%$ of the under 70 age group $(p=0.5101)$. Retention was observed in $17.6 \%$ of patients over 70 and in $24.2 \%$ of those under 70 ( $p=0.7278)$.

Conclusions: There appears to be no significant difference in the outcome of intra-detrusor OnabotulinumtoxinA injections for the treatment of DO with regards to patient age, particularly in rates of efficacy, infection and urinary retention after the procedure. Our findings demonstrate that this therapy may be used without hesitation even in older patients over the age of 70 years.

Financial Funding: None.

\section{Poster \# NM85}

\section{ASSOCIATONS BETWEEN VALIDATED UROLOGY AND PSYCHOLOGY OUESTIONNAIRES IN A WOMEN'S UROLOGY CENTER PRACTİCE}

Michael Ehlert, $\mathrm{MD}^{1}$, Donna Carrico, NP, $\mathrm{MS}^{2}$, Janice Tomakowsky, $\mathrm{PhD}, \mathrm{MPH}^{2}$,

Emily Dove-Medows, CNM, MSN ${ }^{2}$, Jennifer Carty, $\mathbf{M A}^{3}$, Mark Lumley, $\mathrm{PhD}^{3}$, Larry Sirls, $\mathrm{MD}^{1}$, Jamie Bartley, $\mathrm{DO}^{1}$ and Kenneth Peters, $\mathrm{MD}^{1}$

${ }^{1}$ William Beaumont Hospital, Royal Oak, Michigan; ${ }^{2}$ Beaumont Women's Urology Center, Royal Oak, Michigan; ${ }^{3}$ Wayne State University Dept of Psychology, Detroit, Michigan

(Presented by: Michael Ehlert, MD)

Introduction and Objectives: Little is known about the relationship of the Overactive Bladder Questionnaire Short Form (OABq-SF) and Pelvic Floor Distress Inventory (PFDI-20) to the following validated psychology questionnaires: Pain Catastrophizing Scale (PCS), Generalized Anxiety Disorder-7 (GAD-7), Patient Health Questionnaire (PHO-8) and General Social Constraints Scale (GSC). The objective of this study is to present correlations between these questionnaires in women with a variety of urologic and pelvic pain conditions presenting to our multidisciplinary urology clinic.

Methods: A retrospective chart review was completed for patients initially seen from July 2012 to April 2013 in our women's urology center. Prior to their first visit patients are asked to complete the following questionnaires: OABq, PFDI, GAD-7, PHO-8, GSC and PCS (if pain was present). Questionnaire data was compiled and Pearson Correlation Coefficients were calculated for statistical significance using the PFDI and $\mathrm{OABq}$ total scores and subscales and the psychology questionnaire total scores.

Results: 175 of 199 women (88\% of total women seen) completed the GAD-7 and PHO-8 while 125 women (63\%) completed the GSC and PCS. Statistically significant correlations were found between the PFDI total score and all total scores on the psychological questionnaires $(p=0.05$, Table 1$)$. The transformed OABq health related quality of life score (HROOL) and all total scores on the psychology questionnaires were significantly negatively correlated $(p=0.01)$, where higher HROOL scores indicated better quality of life. The PHO8 was the only psychological questionnaire that was significantly correlated with the PFDI and all subscales, as well as the OAB q Symptom Severity and HROOL scores $(p<0.05)$. 
Conclusions: These correlations suggest that pelvic floor symptoms, overactive bladder symptoms and quality of life are significantly correlated with psychological variables such as pain catastrophizing, anxiety, social constraints and depression. This underscores the importance for a multidisciplinary approach to treatment. Further research is needed to develop implications for practice.

Funding: Philanthropic gift from Susan E. Cooper.

\begin{tabular}{|c|c|c|c|c|}
\hline $\begin{array}{l}\text { Table 1: } \\
\text { Pearson's Coefficients }\end{array}$ & $\begin{array}{c}\text { Catastrophizing } \\
\text { (PCS) }\end{array}$ & $\begin{array}{l}\text { Anxiety } \\
\text { (GAD 7) }\end{array}$ & $\begin{array}{l}\text { Depression } \\
\text { (PHQ 8) }\end{array}$ & $\begin{array}{c}\text { Social Constraints } \\
\text { (GSC) }\end{array}$ \\
\hline PFDI 20 & $248^{* *}$ & $.188^{*}$ & $.305^{\star *}$ & $.248^{\star *}$ \\
\hline POPDI 6 & $.305^{* *}$ & .135 & $.218^{*}$ & $.305^{\star *}$ \\
\hline CRADI 8 & .198 & .132 & $.238^{* *}$ & .198 \\
\hline \begin{tabular}{l|l} 
UDI 6 \\
\end{tabular} & .110 & .108 & $189^{*}$ & .110 \\
\hline OABq Symptom Severity & .070 & .146 & $.241^{*+}$ & .070 \\
\hline \multirow[t]{2}{*}{$\mathrm{OABq} \mathrm{HRQOL}$} & $-.304^{* *}$ & $-.294^{* *}$ & $-.424^{* *}$ & $-.304^{* *}$ \\
\hline & \multicolumn{4}{|c|}{${ }^{*} \mathrm{P}<0.01,{ }^{* *} \mathrm{P}<0.05$ (two tailed) } \\
\hline
\end{tabular}

\section{Poster \# NM86}

BULLYING AND ABUSE HISTORY: RELATIONSHIPS WITH PAIN, UROLOGIC SYMPTOMS, EXAM AND CO-MORBIDITIES

Michael Ehlert, $\mathrm{MD}^{1}$, Donna Carrico, NP, $\mathrm{MS}^{2}$,

Emily Dove-Medows, CNM, MSN $^{2}$, Marlene Seltzer, MD $^{1}$, Jamie Bartley, $\mathrm{DO}^{1}$, Jason Gilleran, $\mathrm{MD}^{1}$, Larry Sirls, $\mathrm{MD}^{1}$ and Kenneth Peters, $\mathbf{M D}^{1}$

${ }^{1}$ William Beaumont Hospital, Royal Oak, Michigan; ${ }^{2}$ Beaumont Women's Urology Center, Royal Oak, Michigan

(Presented by: Michael Ehlert, MD)

Introduction and Objectives: Nationally, 25\% of women report a history of abuse or bullying. To date there are no publications on bullying and urologic symptoms, and few relate to abuse and urologic symptoms. The objective is to describe abuse/ bullying history relationships with co-morbidities, pain levels, exam findings, voiding and pelvic floor symptoms in a women's urology center.

Methods: All new patients presenting to our center from July 2012 to April 2013 were included in this retrospective chart review. Patients completed an intake history form, pain ratings in several domains (overall, bladder, pelvic, vulvar on a 0-10 scale) and validated questionnaires prior to their visit. These were reviewed and updated in a 1:1 interview. Data was abstracted by four clinicians. Analyses of Variance (ANOVA) with post-hoc correction for multiple analysis and Pearson's correlation coefficients (ü) were calculated.

Results: 199 patients were identified. $24 \%$ of this group reported each a history of abuse $(\mathrm{N}=48)$ and being bullied $(\mathrm{N}=48) .52 \% \quad(\mathrm{~N}=104)$ reported a bullying history (being bullied, being a bully, witnessing bullying). Those with an abuse history and those abused with a bullying history had significantly higher pain scores in each domain (Table 1). Abuse or bullying history was not associated with being sexually active, OABq total or subscale scores, BMI, levator muscle pain or trigger points. Correlations with comorbidities are strong for smoking history ( $\ddot{u}=.220)$, anxiety $(\ddot{u}=.335)$, IBS $(\ddot{u}=.297)$ and depression $(\ddot{u}=.380)$. There was no significant difference related to bullying on the Pelvic Floor Distress Inventory (PFDI), but significant differences were seen in total score $(p=0.013)$, Pelvic Organ Prolapse Distress Index (POPDI-6), $(p=0.01)$ and ColoRectal-Anal Distress Inventory (CRADI-8) $(p=0.049)$ for those with a history of abuse compared to those without a history of abuse.

Conclusions: A history of bullying or abuse or both significantly correlates with pain scores, pelvic floor symptom distress and several comorbidities. A longitudinal prospective study of women bullied or abused prior to urologic/pain symptoms would contribute to research in this field. Funding: Philanthropic gift from Susan E. Cooper.

\begin{tabular}{|c|c|c|c|c|c|}
\hline Table 1: & $\begin{array}{c}\text { A: } \\
\text { +Bullying; } \\
\text { No Abuse }\end{array}$ & $\begin{array}{c}\text { B: } \\
\text { No Bullying; } \\
\text { Abuse }\end{array}$ & $\begin{array}{c}\text { C: } \\
\text { +Bullying; } \\
\text { +Abuse }\end{array}$ & $\begin{array}{c}\text { D: } \\
\text { No Bullying: } \\
\text { No Abuse }\end{array}$ & $\stackrel{P}{P}$ \\
\hline Overall Pain* & $2.86 \pm 0.382$ & $3.33 \pm 1.202$ & $5.49 \pm 0.534$ & $3.15 \pm 0.495$ & 0.001 \\
\hline Bladder Pain** & $1.42 \pm 0.332$ & $3.60 \pm 1.503$ & $3.97 \pm 0.602$ & $2.13 \pm 0.441$ & 0.001 \\
\hline Pelvic Pain ${ }^{4 \star *}$ & $2.32 \pm 0.421$ & $3.80 \pm 1.200$ & $4.94 \pm 0.552$ & $3.00 \pm 0.505$ & 0.004 \\
\hline
\end{tabular}

\section{Poster \# NM87}

\section{PELVIC PAIN: DO WOMEN WITH A PSYCHIATRIC DIAGNOSIS REPORT MORE PELVIC COMPLAINTS?}

\section{Erik Pattison, $\mathrm{MD}^{1}$, Paholo Barboglio, $\mathrm{MD}, \mathrm{MPH}^{2}$}

and Veronica Triaca, $\mathrm{MD}^{3}$

${ }^{1}$ Dartmouth-Hitchcock Medical Center; ${ }^{2}$ Dartmouth-Hitchcock Medical Center, Lebanon $\mathrm{NH} ;{ }^{3}$ Concord Hospital, Concord NH (Presented by: Erik Pattison, MD)

Introduction and Objectives: Female pelvic pain disorders are a heterogeneous group of diagnoses that present significant diagnostic and therapeutic challenges. A connection between such psychiatric conditions as anxiety and depression and chronic pelvic pain has been reported in the literature. Our objective was to discern if women with psychiatric illness report more pelvic complaints than those without.

Methods: An IRB-approved retrospective review was performed on a cohort of 246 women with the diagnosis of pelvic pain and/or dyspareunia. The Pelvic Floor Distress Inventory (PFDI) and the Pelvic Floor Impact Questionnaire (PFIO) and each of their subcategories were analyzed. Patient history and questionnaire data were extracted from initial visit encounter from the patient's chart. The rating of the pain was assigned based on PFDI-20 question \#20. Score answers of no (0), not at all (1) or somewhat (2) were designated "mild pain." Answers of moderately (3) and quite a bit (4) were designated "severe pain." Continuous data were analyzed using student's t-test, categorical data via the Pearson Chi-Square test and questionnaire outcomes via the Wilcoxon test.

Results: Pelvic pain and/or dyspareunia was present in 246 patients and complete questionnaire data was available for 89 . There was no significant difference between the groups with respect to age, BMI, GP status or menopausal status. Women with psychiatric comorbidity reported significantly more pelvic symptoms in each of the subcategories of the PFDI and PFIO questionnaires. With respect to pain severity, there were 44 with a higher degree of pain and 45 with a milder degree of pain. Women with more severe pain were more likely to have psychiatric illness when compared to those with a milder degree of pain (35/44 versus $25 / 45, p=0.041)$.

Conclusions: Our data indicate that women with psychiatric comorbidities do have a greater degree of pelvic complaints. Furthermore, women reporting severe pelvic pain are more likely to have a psychiatric illness. These findings emphasize the importance of awareness on the part of the physician of the increased likelihood of comorbid psychiatric illness in these patients. As a result, there may be potential for improved outcomes with adequate attention to both conditions. 


\section{Poster \# NM88}

PSYCHOLOGICAL DIFFERENCES AMONG WOMEN WITH PAIN, UROLOGIC, AND GYNECOLOGIC DIAGNOSES IN A WOMEN'S UROLOGY CENTER

Janice Tomakowsky, $\mathrm{PhD}, \mathrm{MPH}^{1}$, Jennifer Carty, $\mathrm{MA}^{2}$, Mark Lumley, $\mathrm{PhD}^{2}$, Donna Carrico, NP, $\mathrm{MS}^{1}$, Michael Ehlert, $\mathbf{M D}^{3}$, Emily Dove-Medows, CNM, MSN $^{1}$, Larry Sirls, $\mathrm{MD}^{3}$, Jamie Bartley, $\mathrm{DO}^{3}$, Jason Gilleran, $\mathrm{MD}^{3}$ and Kenneth Peters, MD $^{3}$

${ }^{1}$ Beaumont Women's Urology Center, Royal Oak, Michigan;

${ }^{2}$ Wayne State University Dept of Psychology, Detroit, Michigan;

${ }^{3}$ William Beaumont Hospital, Royal Oak, Michigan

(Presented by: Janice Tomakowsky, PhD, MPH)

Introduction and Objectives: The present study examined psychological differences among women presenting to a multidisciplinary urology clinic. Of particular interest was how depression, anxiety, social support coping and social constraint (feeling constrained in discussing one's problems) differed by diagnostic clusters reflecting the predominance of urogenital pain, urologic or gynecologic problems. Catastrophizing and pain severity were examined in patients with pain. Understanding psychological differences may prove useful in treatment planning for complex patients.

Methods: Women presenting to a women's urology specialty center from July 2012 to April 2013 completed questionnaires prior to their first clinical exam. Validated measures assessed anxiety, depression, seeking social support, social constraint, catastrophizing and pain (severity and interference with functioning). Patients were divided into pain, urologic or gynecologic groups, determined by primary ICD-9 diagnosis at first clinical evaluation. ANOVAs compared the diagnostic groups on the measures.

Results: 180 of 199 patients completed the questionnaires (90\%). As shown in Table 1, the Pain and Gynecologic groups were similar on most psychosocial measures, but had significantly higher levels of pain severity, pain interference with functioning, social constraint, catastrophizing, helplessness and/or emotional support seeking compared to the Urologic group. The three groups did not differ on anxiety and depression measures.

Conclusions: These findings suggest that patients differ psychologically by diagnostic clusters, though the differences are not reflected in greater anxiety or depression. Patients whose conditions are primarily distinguished by pain and gynecologic problems (who may also have pain) fare worse in terms of catastrophizing, helplessness, pain severity and interference with functioning than those with primary urologic diagnoses. Gynecologic patients also appear more socially constrained. These findings may have implications for treatment planning for each diagnostic cluster and suggest that further investigation into diagnostic differences is of merit in ensuring that the needs of this complex population are met. Clinical Relevance: Female Urology-including Incontinence

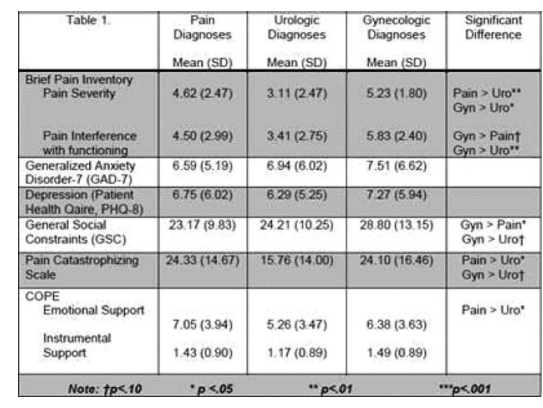

Poster \# NM89

\section{BLADDER PAIN SYNDROME/INTERSTITIAL CYSTITIS IN MALES: CLINICAL PRESENTATION AND CORRELATION BETWEEN SYMPTOMS, CYSTOSCOPIC AND URODYNAMIC FINDINGS.}

Fadi Sawaqed, MD and Jerzy Gajewski, MD FRCSC Department of Urology/Dalhousie University, Halifax/Canada (Presented by: Fadi Sawaqed, MD)

Introduction and Objectives: Bladder Pain Syndrome/Interstitial Cystitis (BPS/IC) is a chronic inflammatory condition of the urinary bladder that is predominantly present in females. Recent publications suggest that BPS/IC is more common in men than expected. It is possible that men with milder symptoms are typically underdiagnosed and often misdiagnosed with chronic nonbacterial prostatitis rather than with early BPS/IC. The aim of this study was review clinical presentation of BPS/IC in men and analyzed any correlations between the symptoms, cystoscopic and urodynamic findings.

Methods: A retrospective chart review was conducted for all male patients with the diagnosis of BPS/IC based on the European Society of the Study of Interstitial Cystitis (ESSIC) criteria between 1995 and 2012. Univariate Analysis of Variance was used to analyze patient's age and the severity of the presenting lower urinary tract symptoms (LUTS) and their correlation to the severity of the cystoscopic findings. The Spearman coefficient was used to define any correlation between the urodynamic parameters, LUTS and the cystoscopic findings.

Results: The study included 75 male patients with a mean age of 45.5 years (SD 12.3) (range 22-78). Suprapubic pain/ discomfort (SPP/D) was the only symptom that is significantly associated with the cystoscopic grade of BPS/IC $(p=0.030)$. There was a significant negative correlation between the maximal cystoscopic capacity and the severity of the incomplete emptying $(p=0.047)$. The maximal cystometric capacity significantly correlates with nocturia (Spearman's rho $=-.482$, $p=0.000$ ). No significant correlations were found between the urodynamic parameters and the cystoscopic findings.

Conclusion: Suprapubic pain/discomfort is the most important LUTS for making the diagnosis of BPS/IC in men. Severity of the SPP/D is significantly associated with degree of glomerulations on cystoscopy. Inverse association was found between the severity of incomplete emptying and the maximal cystoscopic capacity and severity of nocturia and the maximal cystometric capacity.

Financial Funding: None.

Clinical Relevance: IC and Pelvic Pain-UTI / Inflammatory

\section{Poster \# NM90}

\section{A NOVEL COMBINATION OF SURGICAL TECHNIOUES TO RESOLVE LOWER URINARY TRACT EROSION IN A SINGLE OPERATION: NEAR TOTAL TRANSVAGINAL MESH EXCISION, URINARY TRACT RECONSTRUCTION AND CONCOMITANT REPAIR WITH A BIOLOGICAL GRAFT}

Ketul Shah, MD, Andrew Windsperger, MD

and Brian Flynn, $\mathrm{MD}$

Denver, CO

(Presented by: Ketul Shah, MD)

Objectives: To present a single surgeon's management of lower urinary tract mesh erosion using a novel combination of 
surgical techniques to resolve lower urinary tract (LUT) erosion in a single operation. We describe our extensive repair that includes near total transvaginal mesh excision, urinary tract reconstruction and concomitant repair with a biological graft.

Methods: We retrospectively reviewed medical records of 189 patients undergoing transvaginal removal of polypropylene mesh. The focus of this study is 25 patients with polypropylene mesh erosion into the lower urinary tract. We excluded patients with erosion of other foreign bodies into the lower urinary tract $(n=11)$, or mesh that was removed due to isolated vaginal wall exposure $(n=164)$.

Result: 25 patients underwent surgical removal of mesh from the lower urinary tract through a transvaginal approach or combined transvaginal/abdominal approaches. The location of the erosion was the urethra in 14 (56\%) and the bladder in 11 (44\%). Median postoperative length of hospital stay was two (1-6) days. The mean follow up was 21 months all patients (100\%) had complete resolution of the mesh complication in a single operation. There were no major intraoperative complications. Of the patients with urethral erosion a concomitant pubovaginal sling with autologous rectus fascia was performed in 14 of $14 ; 10$ of 14 (71\%) patients were continent while three patients required re-operation due to urethral obstruction. Of the patients with bladder erosion, a concomitant pubovaginal sling with autologous rectus fascia was performed in eight of 11 (73\%); 10 of 11 (91\%) patients were continent while one patient required re-operation due to severe incontinence.

Conclusion: Our extensive repair that includes total or near total transvaginal mesh excision and urinary tract reconstruction can completely resolve lower urinary tract mesh erosion in a single operation. Concomitant pubovaginal sling can be safely and effectively performed at the time of mesh removal in efforts to treat existing stress urinary incontinence (SUI) or avoid future surgery for SUI.

Clinical Relevance: Reconstruction

\section{Poster \# NM91}

\section{SIMPLE VERSUS COMPLEX URETHRAL DIVERTICULUM:} PRESENTATION AND OUTCOMES

Walker Nickles, $\mathrm{MD}^{1}$, Gini Ikwuezunma, $\mathrm{BS}^{2}$, Lara MacLachlan, $\mathrm{MD}^{2}$, Ahmed El - Zawahry, $\mathrm{MD}^{3}$, Ross Rames, $\mathrm{MD}^{4}$ and Eric S. Rovner, $\mathrm{MD}^{4}$

${ }^{1}$ Medical University of South Carolina Charleston, SC; ${ }^{2}$ Southern Illinois University Department of Surgery, Division of Urology, Carbondale IL

(Presented by: Walker Nickles, MD)

Introduction: Urethral diverticulum (UD) may present with a wide range of symptoms and configurations. Though different surgical techniques may be needed for repair depending on the anatomy, it is unclear whether more complex anatomical configurations such as circumferential urethral diverticula (cUD) confer a worse prognosis as compared to simple urethral diverticula (sUD). We reviewed the presentation and operative outcomes of circumferential UD to that of simple UD in order to determine whether the anatomic configuration confers unique presenting symptoms or impacts the surgical outcome.

Methods: Following IRB approval, a retrospective review of patients who underwent transvaginal urethral diverticulectomy at a single institution over an eight year period was performed. A total of 43 patients were identified of which 11 were found to have circumferential urethral diverticula. These lesions were repaired using a previously described technique with complete division of the urethra to access the dorsally located portion of the UD, followed by end-to-end anastomosis for urethral reconstruction. A Martius flap and/or autologous fascial pubovaginal sling (aPVS) were performed as needed. Presenting symptoms and surgical outcomes were reviewed.

Results: Mean age was 51 years in the cUD vs. 52 yo in the sUD. Patients with cUD were more likely to present with SUI compared to those with sUD ( $90 \%$ vs $56 \%, p=0.04$ ) while there was not a statistical difference in the rate of pre-operative urgency, pelvic pain/dyspareunia, post-void dribbling and UTI. In regards to operative management, patients with cUD were significantly more likely to have a concomitant autologous fascial sling (72\% vs $37 \%, p=0.04)$. Post-operatively patients with cUD were more likely to have a UTI ( $27 \%$ vs. $3 \%, p=0.02)$ otherwise rates of post-operative SUI, urgency and retention were similar.

Conclusions: Patients with complex urethral diverticula are more likely to present with SUI and subsequently require an autologous sling at time of repair. Overall, urethral transection and end-to-end anastomosis for the repair of complex urethral diverticula is a feasible approach and results in similar clinical outcomes compared to the repair of simple urethral diverticula.

Clinical Relevance: Reconstruction

\section{Poster \# NM92}

\section{LONG TERM DATA FOR FREE: WHAT CAN BE GAINED FROM A} CLARITY REPORT IN EPIC?

Philippe Zimmern, $\mathbf{M D}^{1}$, Julia Nguyen ${ }^{2}$, Feras Alhalabi, $\mathbf{M D}^{2}$, Josh Youngblood ${ }^{2}$, Dharmendra Pal ${ }^{2}$ and Duwayne Willett ${ }^{2}$

${ }^{1} U T$ Southwestern Medical Center, Dallas, Texas; ${ }^{2} U T$

Southwestern Medical Center

(Presented by: Philippe Zimmern, MD)

Introduction: Electronic medical records (EMR) are here to stay and may have a role in clinical research [1]. Because of the cost, time involved and risk of errors linked to a database specialist transferring clinical data into databases, we studied several processes accessible by EMR using the EPIC system. Here we focused on EMR searches of patient lists against maintained databases.

Methods: With the assistance of our IT team, a Clarity report was prepared in EPIC to search for all patients who had undergone specific urological procedures. A variety of CPT codes were tested to evaluate the patient list's retrieval rate and its accuracy against existing databases going back to 2008. The hypothesis was that EPIC would be equal or superior to our maintained databases.

Results: Table 1 summarizes the findings for seven CPT codes in FPMRS. EPIC searches produced equal or superior listing of patients with the assigned CPT codes, except in cases when more than one CPT code was used or when the billing processed the procedure with the CPT code of interest not as the major CPT code for that case. Accuracy rate was high (>95\%). Clarity report with two CPT codes can be done and provided an excellent overlap in these more complex cases (i.e. CPT code 57287).

Conclusion: Clarity reports using EPIC do provide an equal or superior listing of patients compared to regularly maintained databases for the selected CPT codes in this study. 
Clinical Relevance: Reconstruction

\begin{tabular}{|c|c|c|c|c|}
\hline \multicolumn{5}{|c|}{ Sumumary of patient list searches from $1 / 08$ to $09 / 13$} \\
\hline CPT COOE & ACCESS DATABASE (N) & EPIC RETRIEVAL (N) & RETRIEVAL RATE \% & ACCURACY RATE $\%$ \\
\hline 572895184557240 Anterior Prolopse & 160 & 163 & 98 & 100 \\
\hline 57280 Mesh Sacrocolpopexy & 39 & 45 & 92 & 94 \\
\hline \$7282 Vaginal Voult Fixation & 11 & 13 & 100 & 100 \\
\hline 57250 Rectocele Repparf & 14 & 18 & 100 & 100 \\
\hline 52214 Fulguration Trigonitis & 279 & 302 & 92 & 95 \\
\hline 57287 Sing remoral & 201 & 230 & 87 & 87 \\
\hline 57287 Shing removal + 52214 fulguration & 40 & 45 & $8 s$ & 95 \\
\hline 57287 Sling removal +57283 fascial sling & 4 & 4 & 100 & 100 \\
\hline 57287 Sling removal +51715 injectoble & 20 & 20 & 100 & 100 \\
\hline 51715 Injectable agent & 66 & 6.5 & 100 & 100 \\
\hline
\end{tabular}

\section{Poster \# NM93}

URETHROLYSIS/SLING TAKE DOWN AFTER ROBOTIC SACROCOLPOPEXY \& CONCOMITANT MIDURETHRAL SINTETHIC SLING

Paholo Barboglio Romo, $\mathrm{MD}, \mathrm{MPH}^{1}$ and Veronica Triaca, $\mathbf{M D}^{2}$

${ }^{1}$ Dartmouth-Hitchcock; ${ }^{2}$ Concord Hospital

(Presented by: Paholo Barboglio Romo, MD, MPH)

Introduction and Objective: Concomitant mid-urethral synthetic sling (MUSS) at the time of pelvic organ prolapse (POP) surgery in women without preoperative stress urinary incontinence reduces the likelihood of occult urinary incontinence after reconstructive surgery but increases the likelihood of adverse events (Wei, N Engl J Med 2012). The aim of our study was to evaluate and analyze those women who required urethrolysis or sling take down after robotic sacrocolpopexy (RS) and concomitant MUSS.

Methods: IRB approved single surgeon's retrospective cohort study of women who had RS with concomitant MUSS from April 2010 to October 2012. Outcomes were analyzed at six months. Sling take down or urethrolysis was performed in those women who developed urinary retention requiring clean intermittent catheterization (CIC). We used PFDI-20 and PFIO7 short form questionnaires to evaluate patient subjective data. Pearson's X2, t-student test, Wilcoxon sum ranks test were used for the analysis.

Results: Complete follow up data at six months was available for 140 women. Six women developed urinary retention and underwent urethrolysis/sling take down. All of these stopped CIC and four women had at least 70\% improvement in either PFDI or PFIO-7 afterwards. Patients who required sling take down/urethrolysis had lower body mass index and had lower first sensation volume at the time of preoperatively urodynamics. There was no other significant difference when assessing other pre-op variables.

Conclusion: The rate of post-operative voiding dysfunction and the need for further surgery to address this is low when placing concomitant MUSS at the time of robotic sacrocolpopexy.

Clinical Relevance: LUTS / Voiding Dysfunction

\section{Poster \# NM94}

OUTPATIENT URETHROPLASTY PROVIDES GOOD OUTCOMES FOR URETHRAL STRICTURE REPAIR IN PATIENTS WITH A HISTORY OF FAILED HYPOSPADIAS REPAIR

Andrew Windsperger, $M D^{1}$, Ketul Shah, $M D^{2}$, Dmitriy Nikolavsky, $\mathbf{M D}^{3}$ and Brian Flynn, $\mathbf{M D}^{1}$

${ }^{1}$ University of Colorado, Aurora, CO; ${ }^{2}$ Ohio State University, Columbus, OH; ${ }^{3}$ SUNY Upstate, Syracuse, NY

(Presented by: Andrew Windsperger, MD)
Objectives: We present our experience with outpatient urethroplasty for urethral stricture in patients with failed hypospadias repair and discuss the outcomes.

Methods: We retrospectively reviewed the medical records of patients who had undergone urethroplasty at our institution over a 10 year period. Patients with a history of hypospadias and prior repair with recurrent stricture requiring urethroplasty were included. We excluded patients with other etiologies of urethral stricture as well as hypospadias patients who underwent non-standard urethral repair or repair for reasons other than stricture. Standardized preoperative and postoperative clinical and demographic data were recorded

Results: 204 patients were identified as having undergone urethroplasty at our institution. Of these patients, 29 patients with a history of hypospadias who underwent urethroplasty for urethral stricture repair were identified. Of 51 total procedures, 45 were performed on an outpatient basis and only four surgeries resulted in unplanned hospital admission. Overall, $98 \%$ of patients were discharged after $<23$ hours. 27 of 29 patients were available for follow-up, with overall successful repair of urethral stricture achieved in 23 of 27 patients (85\%). In 16 patients, a planned single-stage repair was performed. Eleven patients underwent multistage repair, and two patients underwent only the first stage of a multistage repair. Primarily successful repair occurred in 18/27 patients, including 6/9 patients who received multistage repair and 10/ 16 patients receiving single stage repair, which improved to 8/9 patients with multistage repair and $13 / 16$ repaired primarily after additional intervention. Persistent complications, defined as fistula, sexual dysfunction or mild cosmetic concerns, existed in five patients at the end of the follow-up period.

Conclusions: Repair of urethral stricture resulting from failed hypospadias reconstruction presents significant challenges for the reconstructive urologist, but repairs are successful in the majority of patients. Outpatient surgery is able to achieve success rates comparable to success rates of hypospadias repair reported in the literature.

Financial Funding: None

Clinical Relevance: Reconstruction

\section{Poster \# NM95}

\section{INFREOUENT COMPLICATIONS ASSOCIATED WITH RESERVOIRS OF UROLOGIC PROSTHETICS, A CASE SERIES AND LITERATURE REVIEW}

Majid Mirzazadeh, MD, Tao Cui, MS and Ryan Terlecki, MD Wake Forest School of Medicine, Winston-Salem, NC (Presented by: Majid Mirzazadeh, MD)

Introduction and Objectives: Complications related to inflatable penile prosthesis (IPP) and artificial urinary sphincter (AUS) reservoirs are rare, potentially life threatening and poorly described in the literature. As more devices are implanted the incidence of complications is likely to increase and it will be important to recognize the signs and symptoms of unusual reservoir related complications.

Methods: We present a case series of reservoir associated complications following IPP and AUS placement at our institution. We also reviewed all current accounts of reservoir associated complications in the literature.

Results: Three cases of rare reservoir associated complications treated in our institution are as follows: Case 1 is passing of an intact AUS reservoir per rectum, after erosion into the cecum, in a patient with a history of myelomeningocele and bladder augmentation. Case 2 involves an IPP reservoir causing vascular compression and leg edema, managed with open exploration 
and repositioning of the reservoir. Case 3 involves erosion of a retained IPP reservoir into the peritoneum and migration to a subhepatic area, which was then removed laparoscopically. Literature review yielded eight additional cases of intestinal erosions, 20 cases of bladder erosions and five cases of vascular compromise. Seven of the nine known cases of intestinal complications of reservoirs have occurred in patients with a history of major abdominal surgeries. Other infrequent complications such as injury to the ureters and inguinal herniation of the reservoirs have also been documented.

Conclusion: Complications involving urologic prosthesis reservoirs, although rare, can have serious implications for patients. A high index of suspicion and familiarity with treatment options are required in order to allow timely diagnosis and appropriate treatment. Patients with previous major abdominal surgeries seem to be more prone to intestinal complications of reservoirs and need special concern.

\section{Poster \# NM96}

\section{FAILURE OF THE INFLATABLE PENILE PROSTHESIS DUE TO ABNORMAL FOLDING OF A LOW PROFILE RESERVOIR}

Tao Cui and Ryan Terlecki

Wake Forest School of Medicine, Winston-Salem, NC (Presented by: Ryan Terlecki)

Introduction: The Conceal Low-Profile reservoir was first launched in February 2011 to much acclaim. Since then, the Conceal reservoir has gained popularity due to its claims of improved cosmesis and ease of implantation, particularly in patients where an ectopic placement is necessary. A literature review does not reveal any specific complications resulting from the conceal reservoir.

Methods: We present two cases of inflatable penile prosthesis failure due to improper folding of the conceal reservoir after implantation.

Results: Case 1 is a 45 year old patient who received an inflatable penile prosthesis and was unable to fully deflate the device postoperatively. CT scan revealed an abnormal fold in the reservoir, causing the reservoir to incompletely fill and empty. Case 2 is a 72 year old patient who's inflatable penile prosthesis did not fill fully. The prosthesis and reservoir was placed without incident and functioned adequately for one year before abruptly failing to fill. CT scan revealed the Conceal reservoir was folded in half, trapping the fluid in the top half. Conclusion: The Conceal reservoir has been generally well received and has been shown to be safe to be placed in either a retroperitoneal or an ectopic location. However, the flexible side walls and the low profile of the Conceal reservoir can cause the device to fold even a year after implantation and causes the IPP cylinders to inflate and deflate incompletely. Further study is needed to determine the incidence and mechanism of this novel complication.

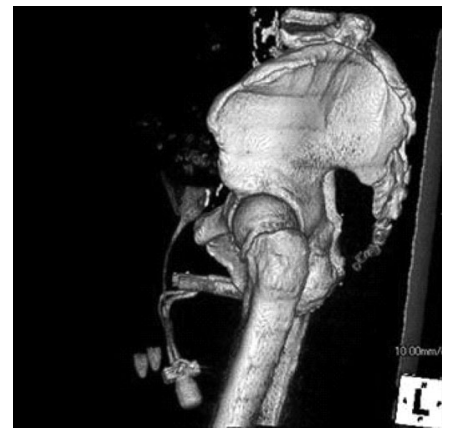

\section{Poster \# NM97}

\section{TRISMUS: AN UNCOMMON COMPLICATION OF BUCCAL} MUCOSAL GRAFTING

Natalie Gaines, $M D$, Joshua Demke, $M^{1}$ and Jonathan Vordermark, MD $^{2}$

${ }^{1}$ Texas Tech University Health Sciences Center Department of Surgery, Division of Facial Plastic and Reconstructive Surgery, Lubbock, Texas; ${ }^{2}$ Texas Tech University Health Sciences Center Department of Urology, Lubbock, Texas

(Presented by: Natalie Gaines, MD)

Introduction: Many modalities exist to create a neovagina, including buccal mucosal grafting (BMG). Only a few minor self-limited oral complications are reported. We report on a patient who underwent buccal mucosa vaginoplasty and developed severe oral scar contractures from her intraoral buccal cheeks to her lateral gingiva-alveolar mucosa with resultant difficult jaw opening, severe class II occlusion and mandibular growth impairment. Scar contracture release required more operations.

Methods: Patient is a female-identifying 16-year-old with ambiguous genitalia and clitoromegaly at birth; karyotype is a 46XY male pseudohermaphrodite. At age 12 she underwent BMG vaginoplasty with progressive development of severe oral contractures. Nine months later she underwent scar excision and Alloderm graft placement. On presentation to the Facial Plastic and Reconstructive surgeon at Texas Tech, she was 2.5 years post-op and her interincisal distance (IID) was $2.5 \mathrm{~cm}$. Repeat scar contracture release with Alloderm skin graft placement to buccal donor sites was done; repeat scar contracture on the right side was treated with local Kenalog injections.

Results: She was poorly compliant with aggressive jaw physiotherapy and six months later had repeat excision with buccal z-plasties to lengthen scarred buccal mucosa. Despite attempts at aggressive physical therapy, her IID decreased to $1.7 \mathrm{~cm}$. Seven months of marginal adherence to physical therapy improved IID to $2.4 \mathrm{~cm}$, although still with significant microstomia/trismus and overjet.

Conclusion: While BMG is associated with a low rate of complications, scar contractures are functionally problematic with mandibular growth restriction. This patient was poorly compliant with physical therapy, an additional challenge. Parents of children undergoing BMG must understand that, while generally safe, it can result in oral scarring. A paucity of literature exists regarding primary defect closure in BMG. Dublin et al. reported that at a mean follow-up of 20.9 mo., 32\% of patients who underwent BMG with primary defect closure for urethroplasty reported subjective tightness. Rourke et al. found that no difference in oral opening existed between urethroplasty patients who underwent BMG with primary closure versus healing by secondary intent after post-operative day 5. No literature addresses children or describes more objective data, such as comparing pre- versus post-operative IID. These areas could be addressed in future studies.

Clinical Relevance: Reconstruction

\section{Poster \# NM98}

ROBOTIC SACROCOLPOPEXY ONE YEAR OUTCOMES: SINGLE SURGEON'S REVIEW OF 127 CASES

Paholo Barboglio Romo, MD, $\mathrm{MPH}^{1}$ and Veronica Triaca, $\mathbf{M D}^{2}$ ${ }^{1}$ Dartmouth-Hitchcock; ${ }^{2}$ Concord Hospital

(Presented by: Paholo Barboglio Romo, MD, MPH) 
Objective: The aim of our study was to review subjective and objective outcomes including complications after robotic assisted laparoscopic sacrocolpopexy (RALS) for the repair of symptomatic pelvic organ prolapse (POP).

Methods: IRB approved, single site retrospective cohort study of women undergoing RALS with concomitant mid-urethral sling, with/without supracervical hysterectomy from April 2010 to October 2012. Outcomes were analyzed at 12 months. Anatomic success was determined by no anterior and apical POP larger than grade one. We used PFDI-20 and PFIQ-7 short form questionnaires to evaluate patient subjective data. We established a strict improvement of $>70 \%$ on questionnaire's total score to determine clinical improvement. Pearson's X2, tstudent test, Wilcoxon sum ranks test were used for the analysis.

Results: Complete follow up data was available for 127 of 140 women who underwent surgery. Eight women had recurrent anterior POP and one of these had associated apical POP at one year. Only one of these desired to undergo further reconstructive surgery. Clinical improvement was present in $72 \%$ by PFIO-7 and in $65 \%$ by PFDI-20. Mesh extrusion occurred in five patients (3.9\%). Other complications reported were bowel injury $(2.4 \%)$, readmission rate $(2.4 \%)$, wound infection $(1.6 \%)$, post-op hernia at port site $(1.6 \%)$ and urethrolysis (3.9\%).

Conclusion: RALS represents a safe and effective surgical therapy to manage symptomatic apical POP. Serious complications rate are low but not rare when assessing outcomes at one year.

Clinical Relevance: Pelvic Organ Prolapse

\section{Poster \# NM99}

\section{PREDICTORS OF REGRET AFTER COLPOCLEISIS FOR PELVIC ORGAN PROLAPSE: A SYSTEMATIC REVIEW}

Juzar Jamnagerwalla, $\mathbf{M D}^{1}$, Karyn S. Eilber, $\mathbf{M D}^{1}$, Samantha Jagannathan, BS ${ }^{2}$, Niv Hakami-Majd, BS ${ }^{3}$, Eugene Shkolyar, $\mathrm{BS}^{3}$ and Jennifer T. Anger, $\mathrm{MD}^{1}$

${ }^{1}$ Cedars-Sinai Medical Center, Los Angeles, CA; ${ }^{2}$ Saint Louis University School of Medicine; ${ }^{3}$ UCLA David Geffen School of Medicine, Los Angeles, CA

(Presented by: Juzar Jamnagerwalla, MD)

Objective: Colpocleisis is a safe and effective procedure to alleviate the symptoms of pelvic organ prolapse (POP) in women who are not sexually active (Fitzgerald et al.). With the goal of determining factors associated with regret after colpocleisis, we synthesized the available literature examining satisfaction and reasons for regret among women undergoing colpocleisis.

Methods: The review was conducted according to the recommendations of the MOOSE (Meta-Analysis of Observational Studies in Epidemiology) group. We performed a systematic review of PubMed and Medline to identify articles published between 1948 and 2013 using search terms "colpocleisis, obliterative and vaginal".

Results: Our literature search identified 182 titles, of which 42 were ultimately included in the review. Twenty-nine studies reported objective POP outcomes data with a pooled patient population of 1,714 patients. Objective POP success ranged from $89 \%$ to $100 \%$. Seventeen studies included satisfaction in their analysis, with a pooled patient population of 1,077 patients. The individual satisfaction reported in these studies ranged from $86.4 \%$ to $96 \%$. Twenty-four studies included data on urinary outcomes after colpocleisis. The rate of de novo stress urinary incontinence (SUI) in women who did not undergo concomitant sling ranged from 0-13\%. In women who had preoperative SUI with concurrent placement of midurethral sling, SUI was persistent in between $6-17 \%$ of women. Ten studies included regret in their analysis, with a pooled patient population of 415 patients. Thirty-one out of 415 women $(7.5 \%)$ at least partially regretted their decision. Frequent causes of regret included loss of sexual function (41\%) and worsening pre-operative urinary incontinence (31\%).

Conclusions: Though colpocleisis is associated with a high success and satisfaction rate overall, few studies exist that examine sources of regret. The data available suggests that regret is often due to worsening UI or remorse about loss of sexual function. Careful patient selection for colpocleisis may help predict who may be more likely to become sexually active again. In addition, patient age alone should not deter providers from placing a concomitant prophylactic sling at the time of colpocleisis.

Clinical Relevance: Female Urology-including Incontinence

\section{Poster \# NM100}

\section{ABDOMINAL SACROCOLPOPEXY USING AUTOLOGOUS FASCIA: INITIAL RESULTS}

Judy M. Choi, $M^{1}$ and Shlomo Raz, $\mathbf{M D}^{2}$

${ }^{1}$ UCLA, Los Angeles, CA; ${ }^{2}$ UCLA Department of Urology, Los

Angeles, CA 90095

(Presented by: Judy M. Choi, MD)

Objectives: Abdominal sacrocolpopexy (ASC) with mesh has been regarded as the most durable operation for advanced pelvic organ prolapse (POP). However, recent reports estimate that there is a $10.5 \%$ risk of mesh erosion at seven years, with either symptomatic or anatomic failure in $34-48 \%$ of cases. There are few alternatives noted in the literature to manage severe mesh complications. We propose a novel technique using a patient's own rectus fascia in lieu of mesh to perform ASC, in the setting of SCP mesh removal.

Methods: After IRB approval was obtained, a retrospective review was performed using medical records of patients referred to our tertiary care center for mesh complications following ASC from May to October 2013. All patients underwent complete ASC mesh removal transabdominally, with concomitant ASC with autologous fascia. A lower midline incision was used to remove previous mesh in toto, and a $10 \times 2 \mathrm{~cm}$ segment of anterior rectus fascia was harvested longitudinally. The fascial segment was then configured into an "L"-configuration; the inferior portion was sutured using \#1-Vicryl sutures to the vaginal cuff, while the tail end was attached to the sacral promontory.

Results: Five patients were included in the study. Mean age was 57.20 years, and mean BMI was 28.0 (range: $22.8-38$ ). Time since original SCP was 52 months (23-76 months). Prior SCP consisted of two open, two robotic and one laparoscopic SCP. The original SCP was performed with polypropylene $(n=3)$ or Prolene mesh $(n=2)$, and all were placed with permanent sutures (1 Prolene, 2 Gore-Tex, 2 Ethibond). Presenting symptoms included dyspareunia (4), vaginal pain (2), vaginal bleeding (3), lower back pain (4), lower abdominal pain (3) and hip pain (30, as well as rectovaginal fistula (1)). On presentation, one had a recurrent cystocele, and another had an enterocele and rectocele. Mesh could be palpated on two patients. During the ASC with autologous fascia, mean EBL was $390 \mathrm{cc}$, and mean hospital length of stay was 5.8 days. All patients reported significant improvement of their original 
symptoms at follow-up (mean 47 days) without any recurrent POP.

Conclusions: This is the first report to address the simultaneous management of severe mesh complications from ASC, and POP following ASC mesh removal. Abdominal sacrocolpopexy using autologous fascia is a safe and feasible alternative to the traditional SCP using mesh although greater follow-up time is necessary to assess its durability and outcomes.

Clinical Relevance: Pelvic Organ Prolapse

\section{Poster \# NM101}

\section{NATIONWIDE TRENDS IN MESH-BASED REPAIR FOR ELDERLY PATIENTS WITH PELVIC ORGAN PROLAPSE}

Tina Schubert, Melissa A. Laudano, MD, Fujun Zhao, MD, Bilal Chughtai, MD and Richard K. Lee, MD Department of Urology, Weill Medical College of Cornell University, New York, NY, USA

(Presented by: Tina Schubert)

Introduction and Objectives: Pelvic organ prolapse (POP) is a common condition in aging women. The use of mesh-based transvaginal repair has recently come under scrutiny, particularly with two Public Health Notifications from the Food and Drug Administration (FDA) in 2009 and 2011. We aim to assess the utilization of mesh-based transvaginal repairs of POP in elderly women over time with respect to these warnings.

Methods: An analysis of the 5\% Medicare Public Use Outpatient Files (years 2001, 2004, 2007-11) was performed to assess changes in the utilization of mesh-based transvaginal prolapse repair surgery. Patients were identified using International Classification of Diseases, 9th Edition (ICD-9) and the Current Procedure Terminology (CPT) codes. Statistical analyses, including the Fisher and $\chi 2$ tests as well as multivariate regression analyses, were performed using SAS 9.3 (SAS Institute Inc., Cary, NC) and SPSS v20 (IBM Corp., Armonk, NY).

Results: The rates of diagnosis and treatment for POP in elderly patients increased significantly by 53\% (68560 to 104760 patients) and 390\% (5420 to 26540 patients), respectively, over the study period ( $p<0.001)$. The use of surgery increased from $52.3 \%$ to $73.9 \%$ while the use of pessary decreased correspondingly $(p<0.001)$. The use of mesh-based transvaginal repair was first seen in 2007 with $28.0 \%(n=2820)$ of patients and then peaked in 2010 with use in $35.4 \%(n=6760)$ of patients. A subsequent decline was seen in 2011 with $30.7 \%(n=6100)$ use (See Table, $\mathrm{p}<0.001$ ). Patients were correspondingly treated with an increase non-mesh repair (64.6 to $69.3 \%$ over the same time period).

Conclusions: The treatment of POP in the elderly has changed over time. The use of surgery has increased. Mesh-based transvaginal repair appeared in 2007, peaked in 2010, and has subsequently begun to decline. FDA warnings regarding the use of mesh grafts appear to be affecting US practice patterns. Clinical Relevance: Pelvic Organ Prolapse

\begin{tabular}{|r|r|r|r|r|r|r|}
\cline { 2 - 7 } \multicolumn{1}{c|}{} & $\begin{array}{c}\text { Total number of } \\
\text { transvaginal } \\
\text { procedures }\end{array}$ & $\begin{array}{c}\text { Mesh-based } \\
\text { Repair }\end{array}$ & $\%$ & $\begin{array}{c}\text { Non Mesh- } \\
\text { Repair }\end{array}$ & $\%$ & p-value \\
\hline 2001 & 3240 & 0.0 & 3240 & 100.0 & \\
2004 & 6360 & 0 & 0.0 & 6360 & 100.0 & \\
2008 & 10080 & 2820 & 28.0 & 7260 & 72.0 & \multirow{2}{*}{$* 0.001$} \\
2009 & 12060 & 4240 & 35.2 & 7820 & 64.9 & $<$ \\
2010 & 16640 & 5760 & 34.6 & 10880 & 65.4 & \\
2011 & 19100 & 6760 & 35.4 & 12340 & 64.6 & \\
\hline Total & 19880 & 6100 & 30.7 & 13780 & 69.3 & \\
\hline
\end{tabular}

Table: Mesh utilization rates in transvaginal repairs for pelvic organ prolapse 2001-2011

\section{Poster \# NM102}

\section{SECONDARY PROCEDURES AFTER ROBOTIC ASSISTED} PROLAPSE SURGERY (RAPS)

Michael Ehlert, MD, Jamie Bartley, DO, Kim Killinger, RN, MSN, Jason Gilleran, MD and Melissa Fischer, MD William Beaumont Hospital, Royal Oak, Michigan (Presented by: Michael Ehlert, MD)

Introduction and Objectives: The safety and efficacy of minimally invasive abdominal prolapse repair has been established. Many patients require secondary procedures for recurrent prolapse, incontinence, mesh complications and other sequelae of laparoscopic abdominal surgery. We examine all secondary procedures after RAPS.

Methods: Consecutive RAPS from 2007-2012 were identified though retrospective chart review. All secondary procedures after primary surgery were categorized.

Results: 100 patients underwent attempted RAPS by three surgeons. Excluding one aborted procedure due to abdominal adhesions and one aborted sling due to a vaginal inclusion cyst, 19 patients underwent 23 subsequent procedures. Primary procedures were sacrocolpopexy (18) and sacrohysteropexy (1). Concomitant procedures included hysterectomy (3), retropubic urethrolysis (1), vaginal hysterectomy (1), removal of prior sacrocolpopexy mesh (1), umbilical hernia repair (1) and antiincontinence procedures (4 TOT slings, 2 Burch colposuspensions, 1 mini-sling). During a mean follow-up of 16 months, seven patients underwent anti-incontinence procedures (6 synthetic slings, 1 fascial sling in a patient after synthetic sling, 3 urethral bulking agents). Secondary prolapse repairs were performed in six patients with recurrent grade 3 prolapse: two anterior repairs with transvaginal mesh, one primary anterior repair with partial excision of prior mesh, two posterior repairs with transvaginal mesh and one primary posterior repair. Other procedures included cystoscopic suture removal with subsequent open mesh excision from bladder, transvaginal suture granuloma excision and transvaginal mesh excision (1 each). Transvaginal urethrolysis with Martius flap was performed for persistent obstructive voiding after the attempted robotic urethrolysis. Additionally, two patients presented with an incarcerated port-site hernia requiring laparotomy, one with a small bowel resection. The patient who had an umbilical hernia repair subsequently underwent laparoscopic ventral wall hernia repair with mesh. Median time to second procedure was 258 days for prolapse repair, 48 days for slings and 249 days for remaining vaginal and abdominal surgeries (range 32025 days).

Conclusions: Robotic assisted prolapse surgery may result in the need for secondary procedures and patients should be counseled appropriately. Adequate follow-up beyond one year is required to monitor this population closely.

Funding: Philanthropic funding MPURE.

\section{Poster \# NM103}

LONG-TERM OUTCOME OF UTERINE SPARING PROCEDURE AS PART OF ANTERIOR VAGINAL WALL SUSPENSION

Burhan Coskun, MD, Rebecca S. Lavelle, MD, Feras Alhalabi, MD, Alana L. Christie, MS and Philippe E. Zimmern, MD

University of Texas Southestern Medical Center, Dallas, Texas (Presented by: Burhan Coskun, MD) 
Introduction and Objectives: We report our experience with anterior vaginal wall suspension (AVWS) for stress urinary incontinence (SUI) and/or moderate cystocele, including hysteropexy for uterine descent less than stage 2.

Methods: Patients undergoing AVWS with uterine preservation and had a one year minimum follow-up were extracted from a long term prospective IRB approved surgical prolapse database. Indication for uterine preservation was a uterine descent not beyond the distal third of the vagina with traction while under anesthesia, and negative PAP smear and pelvic ultrasound pre-operatively. The upper suture of the AVWS secures the cardinal ligament complex, allowing for uterine suspension once the suture is transferred suprapubically (Figure 1). Failure was defined as prolapse recurrence $>$ Stage 2 on physical examination or the need for re-operation for uterine descent. Outcome measures at serial intervals included validated questionnaires, physical examination, standing voiding cystogram at six months post-operatively and complications.

Results: From May 1996 to March 2012, 53 of 739 patients met inclusion criteria. Mean follow up was 55 months (12-175), with mean age 62 years (38-84), mean BMI 27.5 (18.3-49.4) and mean parity 2.7. There were no transfusions or intra-operative complications. Eight patients (15.1\%) underwent subsequent hysterectomy, seven for uterine prolapse recurrence and one for an abnormal PAP smear at 2-6 years post-operatively. Conclusions: The AVWS procedure offers a simple alternative with acceptable long-term follow-up in patients with moderate uterine prolapse who wish for uterine preservation. However, patients should be appropriately counseled about the low risk of subsequent hysterectomy.

Financial Funding: None

Clinical Relevance: Pelvic Organ Prolapse

Table 1: Pre-operative and Last visit (post-operative) questionnaire scores. as well as examination for uterine support and vcuG findings comparing grades of cystocele at pre and 6 months post-operative.

\begin{tabular}{|c|c|c|c|}
\hline & Pre-cperative & Pont-getrasive & Pralue \\
\hline OLCWAS 0-10h, Mean (a SD) & $6.4(0.4)$ & $32(0.5)$ & 0.0001 \\
\hline$[0-7,0.21) \operatorname{Mean}(E \mathrm{SD})$ & $7.8(1.2)$ & $3(0.7)$ & 0.0012 \\
\hline Total UDA-6 (0-16), Mesin (4 SD) & $82(06)$ & $8.4(0.6)$ & 0.0004 \\
\hline Ueerine prolspue, $n$ (\%) & $53(100 \%)$ & $7(13224)$ & \\
\hline \multicolumn{4}{|l|}{ Cribovie vougl: } \\
\hline Grade $1-2(<\mathrm{Scm}) \mathrm{n}$ & 40 & 7 & \\
\hline Grade $3(\mathrm{~s} \operatorname{Sem}) \mathrm{n}$ & 11 & 1 & \\
\hline
\end{tabular}

Figure 1: Schematic of AWWS technique whth uterine preservation

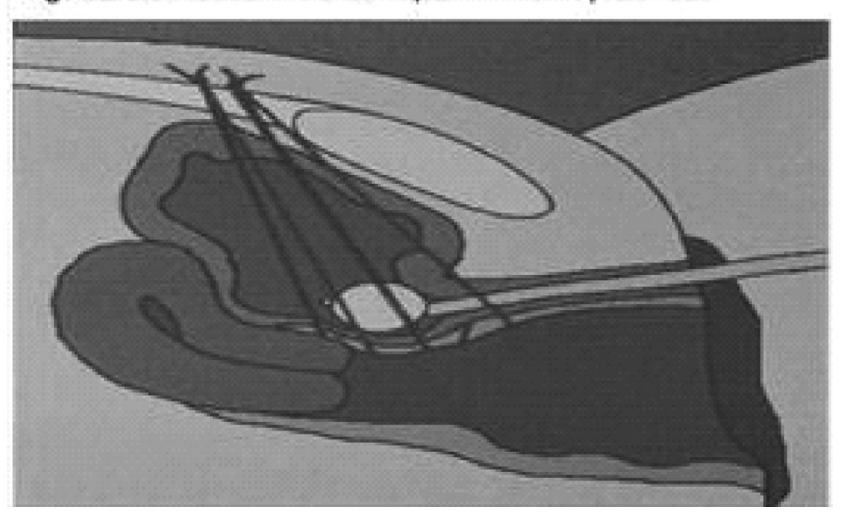

\section{Poster \# NM104}

MESH AUGMENTED POSTERIOR VAGINAL PROLAPSE REPAIR -A CURSE OR A CURE?
Amit Chakrabarty, MD, MS, FRCS, FICS ${ }^{1}$

and Sarika Gupta, MBBS, MS $^{2}$

${ }^{1}$ Center for Continence and Female Pelvic Health; ${ }^{2}$ University College of Medical Sciences, Delhi, India

(Presented by: Amit Chakrabarty, MD, MS, FRCS, FICS)

Introduction and Objectives: The use of synthetic mesh in vaginal floor repair has been a subject of controversy over the past decade with intense partisanship amongst physicians regarding the safety and the necessity of a mesh augmented repair particularly in the posterior compartment. This retrospective study was designed to evaluate the safety, efficacy and feasibility of mesh augmented posterior repair.

Methods: Charts of all patients who underwent a synthetic mesh augmented rectocele repair, by a single surgeon at a single institution from March 2007 till November 2011, were reviewed. Presenting symptoms and stage of prolapse was documented, the latter using International Continence Society Pelvic Organ Prolapse Ouantification (ICS POP-O) system. Operative data included concomitant surgeries, operative time, any intraoperative complications and blood loss. At follow-up, resolution of preoperative symptoms, any new symptoms and patients' satisfaction based on validated Patient Global Impression of Improvement (PGI-I) scale were noted along with vaginal examination findings.

Results: During this period 73 patients, mean age 60 years, underwent rectocele repair using Apogee IntoPro lite (6 patients) or Elevate Apical- Posterior system (67 patients). Constipation (75\%) followed by splinting to empty bowels (48\%) were the commonest symptoms. 13 and 60 patients had Stage II and III rectoceles respectively. Mean operating time was 39 minutes and blood loss was $76 \mathrm{ml}$. There were no intraoperative complications. $87.6 \%$ patients had improvement in symptoms and $91.8 \%$ patients were overall satisfied with the surgery. Median follow up period was 12.3 months (646 months). Two patients (2.73\%) had mesh exposure (type 1 ); one managed with in office excision and the other with minimal excision of exposed mesh in the OR. 10 patients complained of mild to moderate temporary pelvic or rectal pain after surgery and two had dyspauerenuia. No patient required any surgery for pain. There were no cases of recurrent rectocele, however five cystoceles, six enteroceles and one vaginal vault proplase were noted in 10 patients in subsequent follow-up.

Conclusions: Our results confirm the safety and efficacy of Elevate Apical-Posterior mesh and Apogee system in management of rectocele with an acceptable morbidity and complication profile. Long term prospective randomized studies are required to validate these results and authenticate the beneficial role of mesh augmented vaginal prolapse repair.

Clinical Relevance: Reconstruction

\section{Poster \# NM105}

VAGINAL ANTERIOR REPAIR AUGMENTED WITH PORCINE SMALL INTESTINAL SUBMUCOSA GRAFT IN YOUNG WOMEN: SAFETY AND SHORT-TERM OUTCOMES

David Hatcher, $\mathrm{MD}^{1}$, Renuka Tyagi, $\mathrm{MD}^{2}$, Gregory Bales, $\mathrm{MD}^{1}$ and Doreen Chung, MD, FRCSC ${ }^{3}$

${ }^{1}$ The University of Chicago Medical Center-Section of Urology, Chicago, IL; ${ }^{2}$ Weill Cornell Medical Center, New York, NY; ${ }^{3}$ The University of Chicago Medical Center-Section of Urology \& Mount Sinai Hospital, Chicago, IL

(Presented by: David Hatcher, MD) 
Introduction and Objectives: Pelvic organ prolapse (POP) repairs in young women are not well-studied. There is movement away from mesh in vaginal POP repairs due to morbidity such as erosion. Xenografts may improve outcomes with less morbidity. Few studies exist on their use for vaginal repairs and even fewer on Surgisis ${ }^{\circledR}$ (SIS) (Cook Surgical, porcine small intestinal submucosa). The aim was to determine safety, efficacy and effect on sexual function of anterior vaginal repair with SIS.

Methods: 52 women with $\mathrm{POP}-\mathrm{O} \geq$ stage II anterior POP underwent paravaginal repair with SIS (January 2011-June 2013). Apical, posterior and stress urinary incontinence (SUI) procedures were done as indicated. Standardized follow-up was at one, three, six, one and 12 months. Primary outcomes were anatomic $(\mathrm{Ba}<-1)$ and subjective success (no bulge or pelvic pressure), sexual function (PISO-12), and complications. Results: Mean age was $46 \pm 11$ years. 29 (56\%) had stage 2, 22 (42\%) stage 3 and one (2\%) stage 4 POP. 34 (67\%) were sexually active. $43(82 \%)$ had concomitant apical and $36(69 \%)$ posterior repair. 50 (96\%) had a procedure for SUI. Mean blood loss was $165 \pm 96 \mathrm{~mL}$. Mean total operative time was $148 \pm 68$ minutes for all procedures with mean hospital stay $1 \pm 1$ night. There were no Clavien $\geq$ III complications. Three patients had blood transfusion postop, One after hysterectomy. Eight (15\%) had graft exposure treated conservatively. At last follow-up, overall subjective and objective success rates were $94 \%$ and $81 \%$. In the subset $\leq$ age $45(n=29)$, they were $100 \%$ and $90 \%$. No patients underwent surgery for recurrence or revision. Four reported de novo pelvic pain after three months postop, all age $<45$. Of those sexually active, one $(2 \%)$ reported de novo dyspareunia beyond three months. Of the eight with dyspareunia preop (all $\leq 45)$, four $(50 \%)$ reported complete resolution. Conclusions: Vaginal anterior repair with SIS is safe and effective with low morbidity and little effect on sexual function, particularly in young women. However, longer follow-up and comparative studies are needed.

No disclosures.

\begin{tabular}{|l|l|l|l|l|}
\hline & $\begin{array}{l}1 \text { month } \\
(\mathrm{N}=52)\end{array}$ & $\begin{array}{l}3 \text { months } \\
(\mathrm{N}=37)\end{array}$ & $\begin{array}{l}6 \text { months } \\
(\mathrm{N}=26)\end{array}$ & $\begin{array}{l}12 \text { months } \\
(\mathrm{N}=14)\end{array}$ \\
\hline Objective success all (\%) & 96 & 89 & 85 & 64 \\
\hline Objective success age $45(\%)$ & 100 & 100 & 100 & 88 \\
\hline Subjective success all (\%) & 98 & 92 & 96 & 100 \\
\hline Subjective success age $\$ 45(\%)$ & 100 & 100 & 100 & 100 \\
\hline
\end{tabular}

\section{Poster \# NM106}

\section{DOES URINALYSIS PREDICT MESH EROSION/PERFORATION?}

Justin Ellett, $\mathrm{MD}$, PhD, Lara MacLachlan, $\mathrm{MD}^{1}$, Kelly Johnson, $\mathbf{M D}^{1}$, Gini Ikwuezunma ${ }^{1}$, Michelle Koski, $\mathbf{M D}^{2}$, Ross Rames, $M^{1}$ and Eric Rovner, $M^{1}$

${ }^{1}$ MUSC, Charleston, SC; ${ }^{2}$ Kaiser, San Diego, CA

(Presented by: Justin Ellett, MD, PhD)

Introduction: Urinalysis (UA) has been suggested as a predictor of mesh/perforation erosion into the genitourinary tract. An abnormal urinalysis noted postoperatively following surgical recovery from mesh placement for stress incontinence or prolapse repair may suggest urinary tract perforation. We set out to determine the utility of urinalysis in evaluating patients as a predictor of mesh erosion.

Methods: We retrospectively analyzed 191 patients who eventually underwent transvaginal mesh excision over a six year period from 2007 to 2013 with respect to urinalysis, cystoscopy findings and symptoms at initial presentation prior to mesh explantation. 28 of 191 (14.7\%) patients were found to have mesh eroding into the urinary tract. 57 of 191 (29.8\%) patients had exposure of their vaginal mesh. Six patients had both an erosion and exposure. The remaining patients had neither exposure nor erosion and underwent explantation for other reasons, including bladder outlet obstruction and pain. Urinalysis at initial presentation was evaluated for this study, with the finding of red blood cells, white blood cells, blood or leukocyte esterase being interpreted as a positive UA.

Results: $60 \%$ of the 191 patients had a positive UA. $64 \%$ of patients with erosion had a positive UA, while $52 \%$ of patients with exposure had a positive UA. $27.6 \%$ of patients with neither erosion nor exposure had a positive UA. Importantly, UA was found to be completely negative in 10 of 28 (35\%) patients with erosion and 27 of 57 (47\%) patients with exposure and in 82 of 112 (73\%) patients with other mesh related problems. Of all patients presenting with mesh related complications, the positive predictive value, negative predictive value, sensitivity and specificity of a positive UA are (respectively): PPV $-36 \%$, NPV $-89 \%$, sensitivity $-56 \%$ and specificity $-73 \%$.

Conclusions: In patients with a negative UA and mesh related complication, the likelihood that mesh erosion will not be present is $89 \%$. However, given that $36 \%$ of patients with an erosion had a negative UA, and that the positive predictive value of a positive UA in predicting mesh erosion was only $36 \%$, using UA alone as a predictor of erosion is inappropriate in patients with voiding symptoms following mesh implantation.

Clinical Relevance: Reconstruction

\section{Poster \# NM107}

\section{FACTORS ASSOCIATED WITH INTRAOPERATIVE CONVERSION} DURING ROBOTIC SACROCOLPOPEXY

Brian Linder, MD, Lindsay Hertzig, MD, George Chow, MD, Marisa Clifton, MD and Elliott Daniel, MD

Mayo Clinic, Rochester, $M N$

(Presented by: Brian Linder, MD)

Objective: To evaluate for potential predictors of intraoperative conversion from robotic sacrocolpopexy (RSC) to open abdominal sacrocolpopexy.

Methods: We identified 83 consecutive patients from 2002 to 2012 with symptomatic high-grade post-hysterectomy vaginal vault prolapse that underwent RSC at Mayo Clinic. Multiple clinical variables including patient age, comorbidities (bodymass index [BMI], hypertension, diabetes mellitus, tobacco use), prior intra-abdominal surgery and year of surgery were evaluated for potential association with conversion.

Results: Overall, 14/83 cases (17\%) required conversion to an open sacrocolpopexy. Patients requiring conversion were found to have a significantly higher BMI compared to those who did not (median $30.2 \mathrm{~kg} / \mathrm{m} 2$ versus $25.8 \mathrm{~kg} / \mathrm{m} 2 ; \mathrm{p}=0.003$ ). Other clinical factors evaluated were similar between the cohorts. When stratified by increasing BMI, conversion remained associated with an increased BMI. That is, conversion occurred in $3.8 \%(1 / 26)$ of patients with BMI $\leq 25 \mathrm{~kg} / \mathrm{m} 2,14.7 \%$ (5/34) with BMI $25-29.9 \mathrm{~kg} / \mathrm{m} 2$ and $34.7 \%$ (8/23) with BMI $\geq 30 \mathrm{~kg} / \mathrm{m} 2 \quad(\mathrm{p}=0.004)$. When evaluated as a continuous variable, BMI was also associated with a significantly increased risk of conversion to an open procedure (OR 1.18, $\mathrm{p}=0.004$ ).

Conclusions: A higher BMI was the only clinical factor associated with intra-operative conversion during robotic sacrocolpopexy. Recognition of this may aid in pre-operative counseling and surgical patient selection. 


\section{Poster \# NM108}

ROBOTIC SACROCOLPOPEXY OUTCOMES: IS THERE A CORRELATION BETWEEN OBJECTIVE AND SUBJECTIVE RESULTS?

Paholo Barboglio Romo, $\mathrm{MD}, \mathrm{MPH}^{1}$ and Veronica Triaca, $\mathrm{MD}^{2}$

${ }^{1}$ Dartmouth-Hitchcock; ${ }^{2}$ Concord Hospital

(Presented by: Paholo Barboglio Romo, MD, MPH)

Objective: The aim of our study was to correlate anatomic (objective) outcomes to subjective results from validated questionnaires at one year in women who underwent robotic sacrocolpopexy (RS) for the repair of symptomatic pelvic organ prolapse (POP).

Methods: IRB approved single site retrospective cohort study of women who had RS with concomitant mid-urethral sling, with/without supracervical hysterectomy from April 2010 to October 2012. Outcomes were analyzed at 12 months. Anatomic recurrence was determined by either anterior and/or apical POP larger than grade one. We used PFDI-20 and PFIO-7 short form questionnaires to evaluate patient subjective data. We established a strict improvement of $>70 \%$ on both questionnaire's total score to determine clinical improvement. Pearson's correlation was utilized to calculate correlation coefficient.

Results: Complete follow up data was available for 127 of 140 women who underwent RS. Eight women had recurrent anterior POP and one of these had associated apical POP at one year. Clinical improvement in both questionnaires was present in 105 women (83\%). Only one patient with anatomical recurrence and poor outcomes in both questionnaires underwent further reconstructive surgery. Correlation coefficient was 0.05 between objective and subjective outcomes. When assessing perioperative variables, women who had anatomical failure had worse pre-operative and post-operative symptoms (PFIQ-7) when compared to with no anatomical recurrence.

Conclusion: Women with both anatomical POP recurrence and poor subjective outcomes may undergo further surgical repair despite no correlation between objective and subjective results. Clinical Relevance: Reconstruction

\section{Poster \# NM109}

THE USAGE OF SMALL INTESTINAL SUBMUCOSA EXTRACELLULAR MATRIX (SURGISIS) IN THE TREATMENT OF PELVIC ORGAN PROLAPSE

Vannita Simma-Chiang, MD, Melissa Laudano, MD, Renuka Tyagi, MD

Department of Urology, Weill Medical College of Cornell

University, New York, NY

(Presented by: Melissa Laudano, MD)

Introduction and Objectives: Pelvic organ prolapse (POP) is a common problem affecting up to $40 \%$ of women. In an attempt to improve surgical repairs, various materials to strengthen pelvic floor tissues have been utilized. Given recent scrutiny surrounding the use of transvaginal mesh for POP repairs, alternatives have been explored. We sought to evaluate our anatomical outcomes and complications associated with the use of Surgisis, a biological graft derived from porcine small intestine, in the anterior repair of prolapse patients.

Methods: A retrospective review of 49 patients who underwent anterior repair with Surgisisÿ $£$ (Cook Surgical, Bloomington, IN) between 2009 and 2012 at a single institution by a single surgeon was performed. All patients had at least six months of follow-up. Objective success rates using the pelvic organ prolapse quantification ( $\mathrm{POP}-\mathrm{O}$ ) system and subjective success rates were obtained. Complications were classified and coded according to the International Urogynecological Association and International Continence Society (IUGA/ICS) Terminology and Classification of Complications Related Directly to Grafts in Female Pelvic Floor Surgery.

Results: Mean age \pm SD was $55.2 \pm 10.8$ years. Mean parity was $2.4 \pm 1.5$ and mean BMI was $24.8 \pm 3.4$. Pre-operatively, the mean POP-O score of patients was $2.84 \pm 0.5$. Mean surgical time was $164.1 \pm 70.0$ minutes. With a mean follow-up of $14.7 \pm 8.9$ months following surgery, the mean POP-O score was $1.10 \pm 1.1$. Postoperatively, $87.8 \%$ (43/49) patients subjectively reported improvement of symptoms. In terms of complications, 6/49 (12.2\%) had a complication identified with the IUGA/ICS Classification. Complications included urinary retention, dyspareunia and recurrent UTIs. Of the 49 patients, four $(8.2 \%)$ had an anatomical recurrence, defined as POP-O score greater than or equal to their pre-operative POP-O score. One of these patients elected a repeat surgery, two elected observation and one elected placement of a pessary.

Conclusions: Our outcomes with Surgisis suggest that it is a safe, effective material for anterior pelvic repairs. It is associated with minimal complications and an $8.2 \%$ anatomical failure rate. Long-term follow-up is warranted in order to further assess its durability.

\section{Poster \# NM110}

TRANSVAGINAL RECTOCELE REPAIRS USING MESH: A RETROSPECTIVE REVIEW OF COMPLICATIONS

Priyanka Gupta, MD, Nissrine Nakib, $\mathbf{M D}^{1}$, Kimberly Burgess, $\mathbf{M D}^{2}$ and Suzette E. Sutherland, $\mathbf{M D}^{2}$

${ }^{1}$ Department Urologic Surgery, University of Minnesota, Mpls, MN; ${ }^{2}$ Metro Urology, Centers for Continence Care and Female Urology, Mpls/St. Paul, MN

(Presented by: Priyanka Gupta, MD)

Introduction and Objectives: With the recent FDA warnings about the use of mesh in vaginal surgery, concern about the risks associated with transvaginal mesh procedures has increased, especially with respect to the posterior compartment. The objective is to identify complications associated with vaginal rectocele repairs using mesh.

Methods: The records of two fellowship trained female pelvic floor surgeons were reviewed from 2009-2012 identifying patients that had undergone transvaginal rectocele repairs with mesh. Data collection included patient age, grade of prolapse, symptoms, mesh type, follow-up and specific characteristics of complications.

Results: 80 patients underwent transvaginal rectocele repairs with mesh using Elevate in 73 (91.25\%\%), Exair in five (6.25\%), Prolift in one (1.25\%), and Avaulta in one (1.25\%). Average age was 64 yrs (range 39-88). 43/80 (54\%) patients underwent a posterior mesh repair only, while 37/80 (46\%) underwent combined posterior/anterior/apical mesh repairs. Preoperative grade was GI in six (7.5\%), GII in 37 (46.25\%), GIII in 29 (36.25\%), and GIV in seven (8.75\%). All patients were symptomatic with vaginal bulge, constipation, stool trapping, splinting and/or pelvic pressure. Mean follow-up was 10.6 months (range 0.4 to 36$)$. Overall complication rate was $22 \%$ (18/80), with most deemed minor. Specific mesh-related complications were seen in only $10 \%$ of patients (8/80). Complications included hematoma in $2 / 80$ cases $(2.5 \%)$, intra-operative rectal injury in 
$1 / 80$ (1\%), posterior vaginal mesh exposure in $8 / 80$ (10\%), UTI in $3 / 80(4 \%)$, transient de novo dyspareunia in $1 / 80$ (1\%) and de novo pelvic pain in $2 / 80$ (2.5\%). One pain patient complained of sacral and coccyx pain (later identified as being associated with bulging disc injury) and the other had initial exacerbation of chronic right hip pain, which resolved following manual therapy. Vaginal mesh extrusions were managed with simple office excision and the use of estrogen cream in all cases except one, which did require excision in the operating room. No patients developed prolapse recurrence in the posterior compartment and all had resolution of their related symptoms at the time of follow-up.

Conclusions: In the appropriately selected patient vaginal mesh can be safely used for rectocele repair with good success and minimal complications. Specific mesh-related complication rates are low and most often involve incision-related mesh extrusions, which can usually be handled via office excision and topical estrogen.

Clinical Relevance: Pelvic Organ Prolapse

\section{Poster \# NM111}

\section{MANAGEMENT OF RECURRENT PROLAPSE AFTER ROBOTIC-ASSISTED PROLAPSE SURGERY (RAPS)}

Michael Ehlert, MD, Jamie Bartley, DO, Kim Killinger, RN, MSN, Jason Gilleran, MD and Melissa Fischer, MD

William Beaumont Hospital, Royal Oak, Michigan

(Presented by: Michael Ehlert, MD)

Introduction and Objectives: Recurrent prolapse can occur after abdominal prolapse repair. Treatment options include observation or additional surgical repair. We examine the incidence and treatment of prolapse after RAPS.

Methods: RAPS performed between 2007 and 2012 were identified through retrospective chart review. Patients who were found to have any postoperative prolapse, defined as $\geq$ grade 2 in any compartment, were examined as a subset. Subsequent management, either observation or surgical, was characterized.

Results: Of 100 patients who underwent attempted RAPS by three urologists, 21 had recurrent prolapse. In this subset, 16 had prior hysterectomy, seven had prior anti-incontinence procedure and seven had prior prolapse repair. Preoperative prolapse was characterized as grade $2(1)$, grade $3(11)$, grade 4 (8). 12 had all three compartments involved. The majority received a robotic sacrocolpopexy $(n=19)$, four with concomitant robotic hysterectomy and one had vaginal hysterectomy. Two additional patients had a sacrohysteropexy. With a mean follow-up of seven months, seven patients had grade 3 recurrent prolapse (4 anterior and 3 posterior compartment) and 14 grade 2 (15 Anterior, 11 posterior, and 3 apical). Six patients with grade 3 prolapse underwent a second procedure at an average of 230 days (range 38-345). Procedures included primary cystocele (2) or rectocele repair (1), posterior vaginal mesh (2) and anterior mesh repair (1). None of the grade 2 prolapse required surgical repair.
Conclusions: Recurrent prolapse can occur after robotic assisted prolapse repair. Grade 2 recurrence can successfully be managed conservatively but grade 3 recurrences often require additional surgery. Grade 3 anterior and posterior compartment failures were effectively addressed using a vaginal approach.

Financial Funding: Philanthropic funding MPURE.

\section{Poster \# NM112}

CONCOMITANT HYSTERECTOMY AT TIME OF UTEROSACRAL LIGAMENT FIXATION (USLF) MAY BE ASSOCIATED WITH IMPROVED CLINICAL OUTCOMES AT 3 MONTHS, COMPARED TO THOSE UNDERGOING USLF POST-HYSTERECTOMY

Judy M. Choi, $\mathrm{MD}^{1}$, Rose Khavari, $\mathrm{MD}^{2}$ and Sophie Fletcher, $\mathrm{MD}^{3}$

${ }^{1}$ UCLA, Los Angeles, $C A ;{ }^{2}$ The Methodist Hospital, Houston, TX; ${ }^{3}$ Kaiser Permanente, Santa Rosa, CA

(Presented by: Judy M. Choi, MD)

Introduction and Objectives: Uterosacral ligament fixation (USLF) for vaginal vault suspension has been used successfully to treat symptomatic patients with pelvic organ prolapse. However, few reports have compared clinical outcomes in patients undergoing USLF with concomitant hysterectomy versus those who are post-hysterectomy. We evaluated the effect of concomitant versus prior hysterectomy on complication rates following uterosacral ligament fixation.

Methods: After IRB approval was obtained, we retrospectively reviewed medical records of patients undergoing uterosacral ligament fixation at a multidisciplinary facility over a threeyear period.

Results: A total of 51 patients were included in this study, all of whom underwent uterosacral ligament fixation for the treatment of pelvic organ prolapse. Of these, 38 (74.5\%) underwent transvaginal hysterectomy at the time of USLF and 13 (25\%) had previously undergone hysterectomies. There was no difference in age, pre-operative POP-O scores or follow-up times, and groups underwent similar rates of sling placements and anterior and posterior colporraphies. There were no differences in complication rates between groups either intraoperatively or at three weeks. However, at three months, those who had undergone a hysterectomy prior to USLF reported worse outcomes than those who had undergone concomitant hysterectomy ( $30.8 \%$ versus $13.2 \%, p=0.013$ ). Two patients reporting urge incontinence, one reporting dyspareunia and another reporting difficulties with defecation. Post-operative POP-O scores were evaluated, with similar improvements between groups. Re-operation rates were not found to differ significantly.

Conclusions: Longer follow-up time is needed to fully determine the impact of hysterectomy timing in relation to USLF. However, our preliminary data suggests that concomitant hysterectomy at time of USLF may be associated with improved clinical outcomes in the short-term, compared to those undergoing USLF post-hysterectomy.

Clinical Relevance: Pelvic Organ Prolapse 